\title{
ESTUDIO EXPERIMENTAL DEL EFECTO MAGNUS EN CUERPOS CILÍNDRICOS DE SECCIONES TRANSVERSALES DIVERSAS
}

\author{
Tesis de Doctorado
}

\author{
SANTIAGO PEZZOTTI \\ Presentada ante la Facultad de Ingeniería de la \\ Universidad Nacional de La Plata \\ como requisito para la obtención del grado académico de

\section{DOCTOR EN INGENIERÍA}

Dirección de la tesis:

Dr. Sanz Andrés Ángel, Dr. Nadal Mora Vicente, Dra. Pérez Grande Isabel.

Jurado de la tesis:

Dr. Lassig Jorge, Dr. Sacco Carlos, Dr. Elaskar Sergio.

Fecha de la defensa oral y pública: 26 de abril de 2016. 



\section{ÍNDICE}

NOMENCLATURA

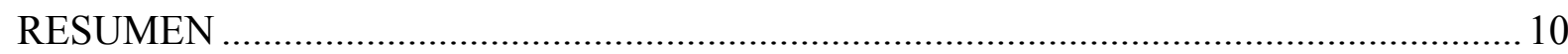

CAPÍTULO 1

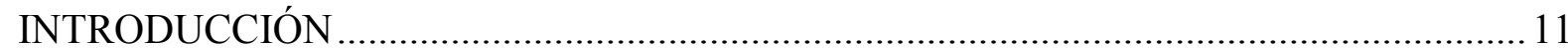

1.1 INTRODUCCIÓN AL ESTUDIO EXPERIMENTAL DEL EFECTO MAGNUS 11

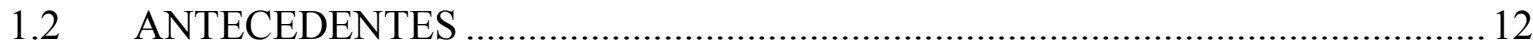

1.2.1 Primeros estudios, cilindros de sección circular............................................. 12

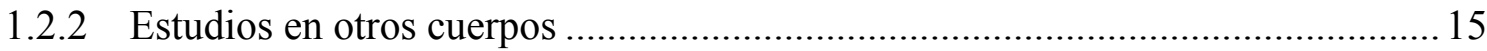

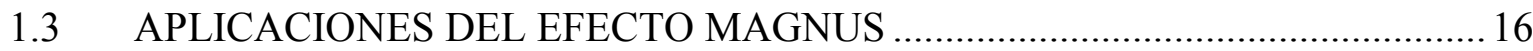

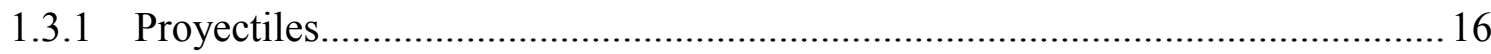

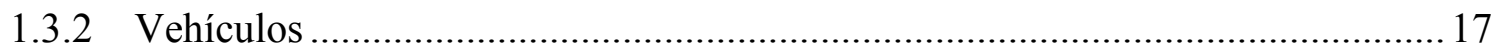

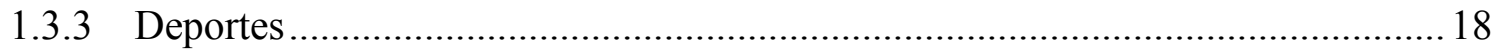

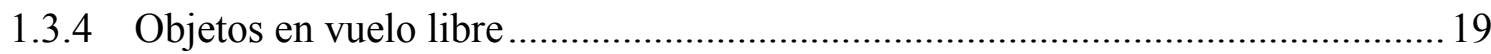

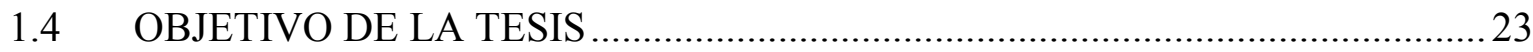

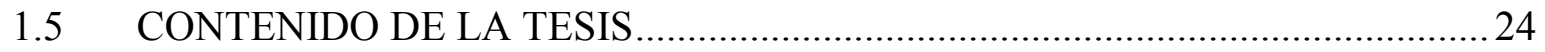

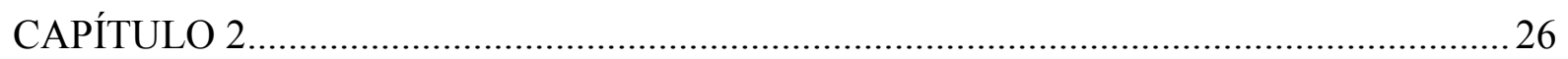

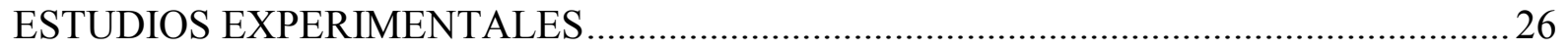

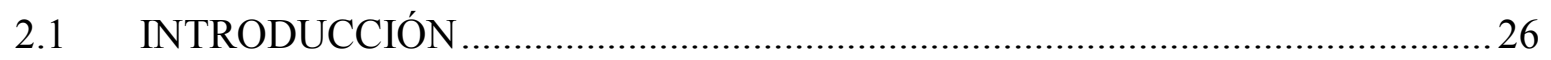




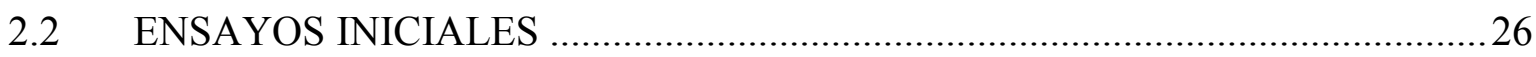

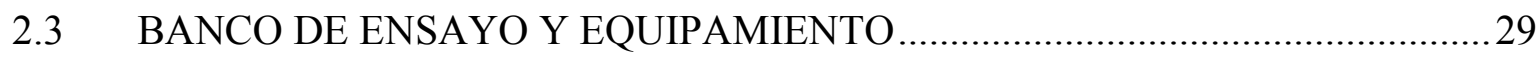

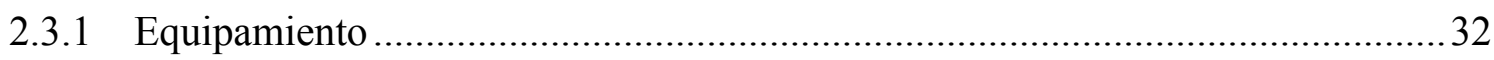

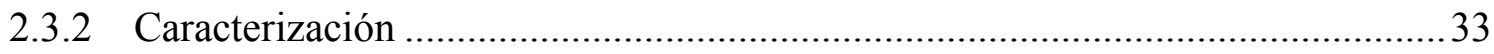

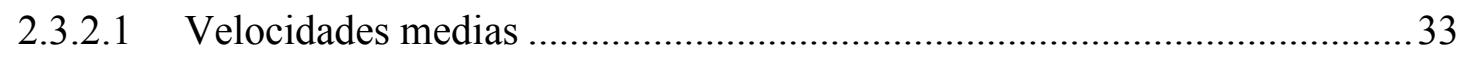

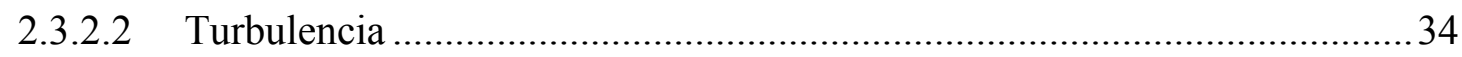

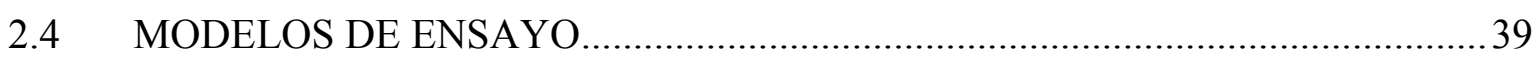

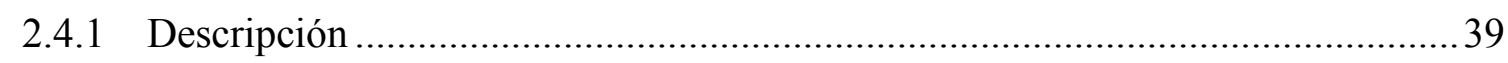

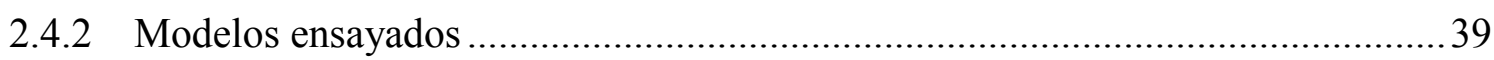

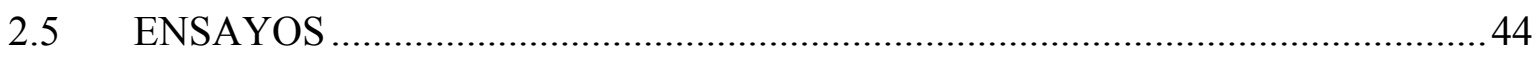

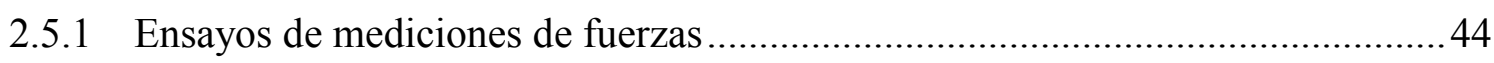

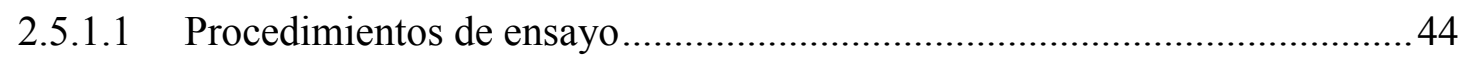

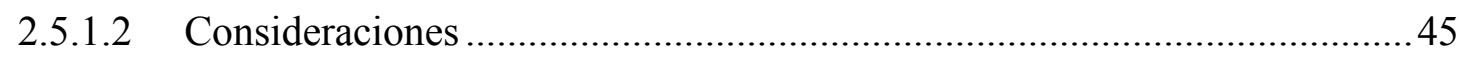

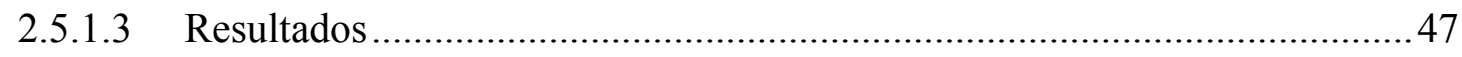

2.5.2 Ensayos de visualización de flujo alrededor de los cuerpos .............................52

2.5.2.1 Equipamiento y características de las fotografías .....................................53

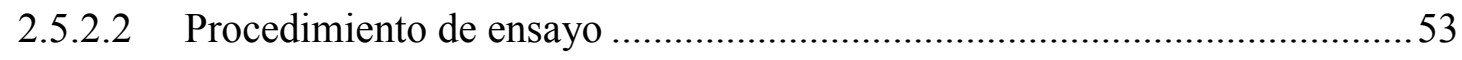

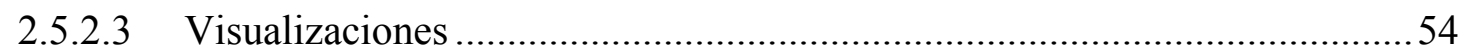

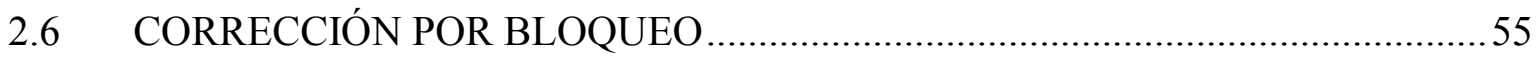

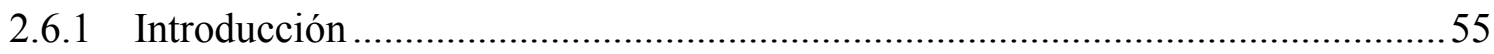

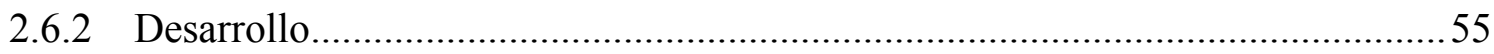




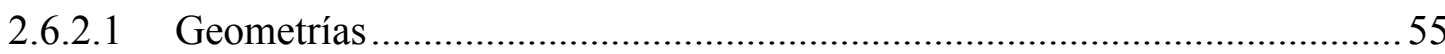

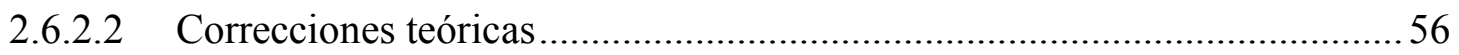

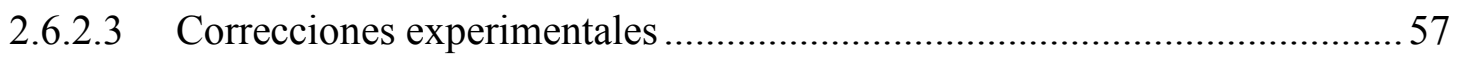

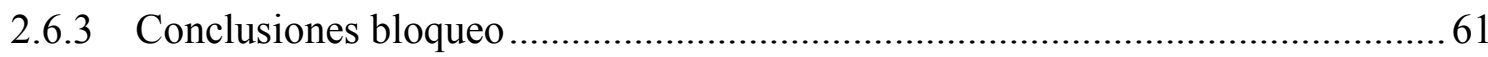

2.6.4 Comparación de resultados con modelos de la bibliografía ............................... 62

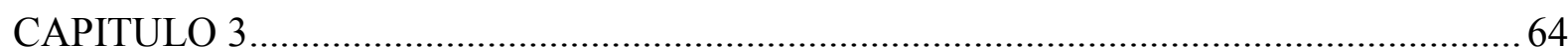

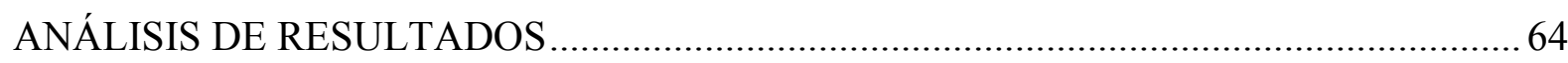

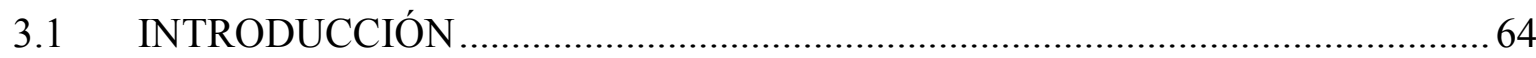

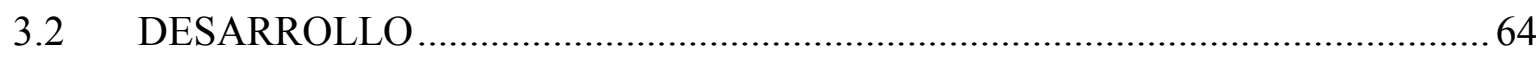

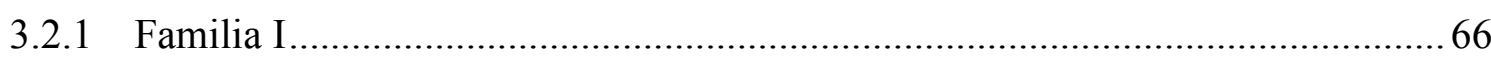

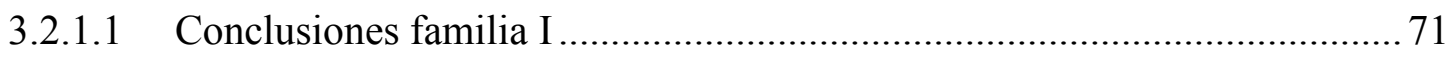

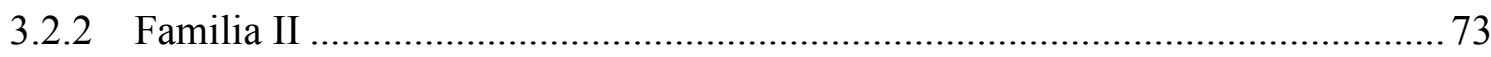

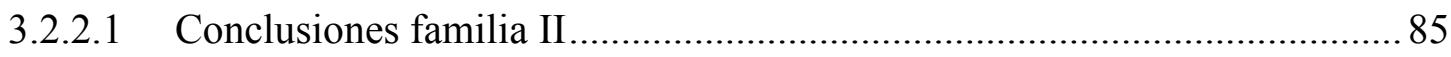

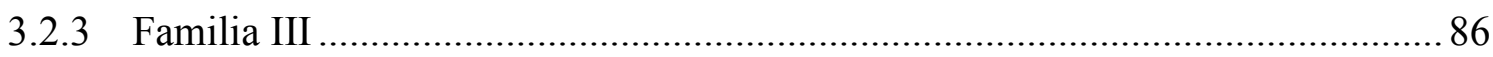

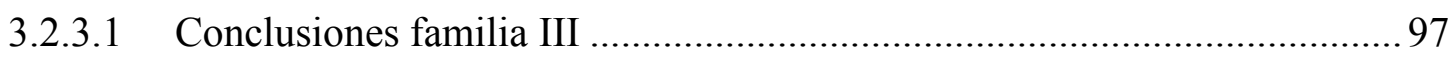

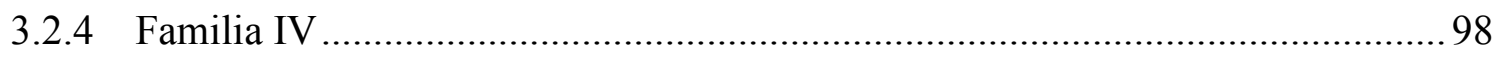

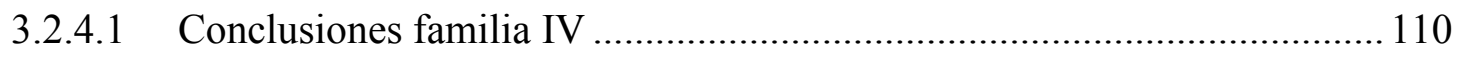

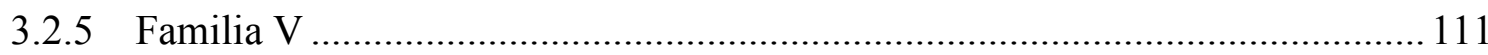

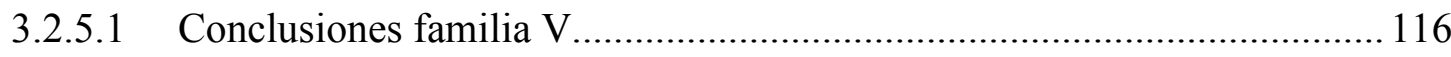

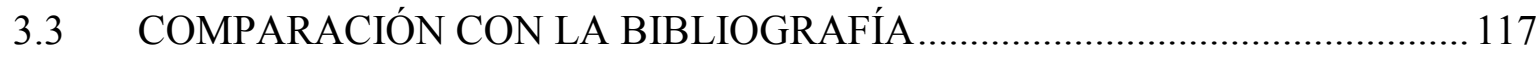

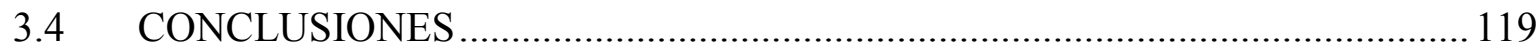


CAPÍTULO 4.

ANÁLISIS TEÓRICO

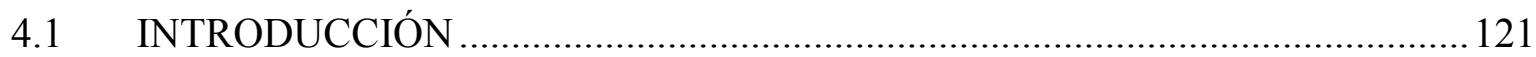

4.2 APROXIMACIONES TEÓRICAS PUBLICADAS …………………………......121

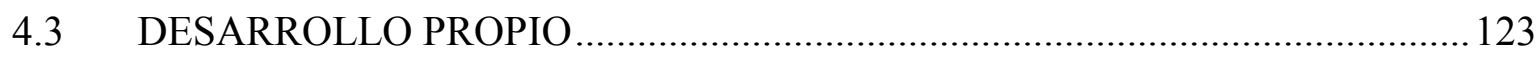

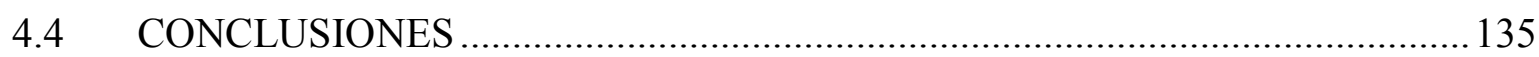

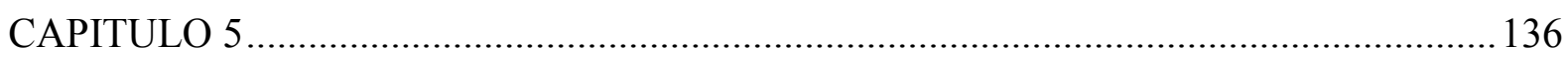

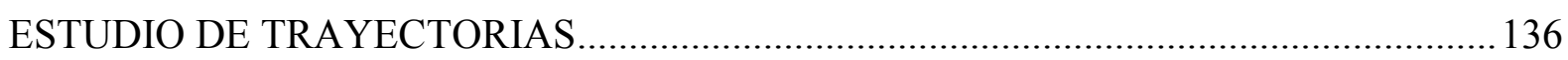

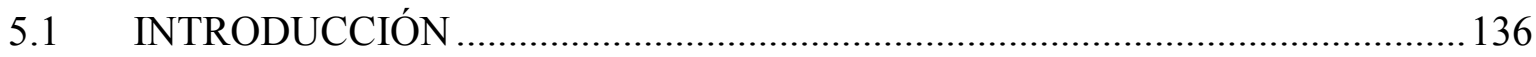

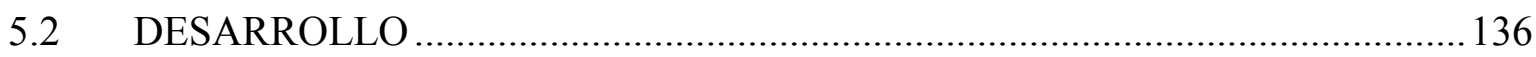

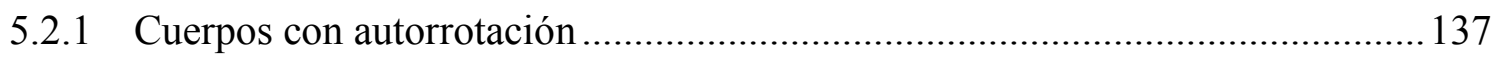

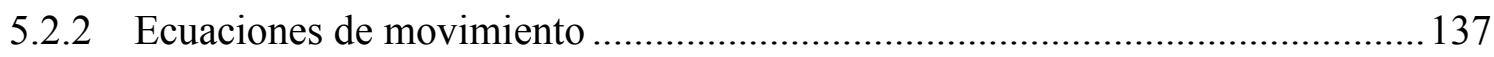

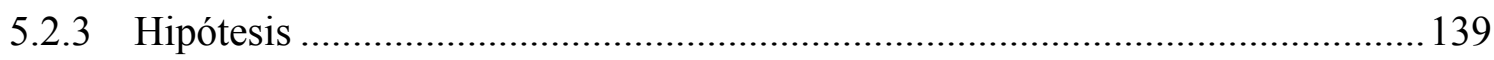

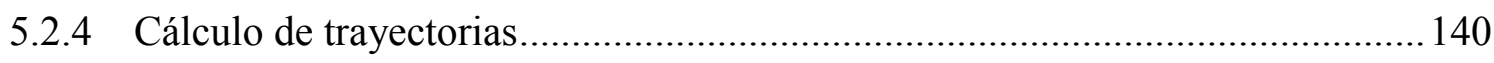

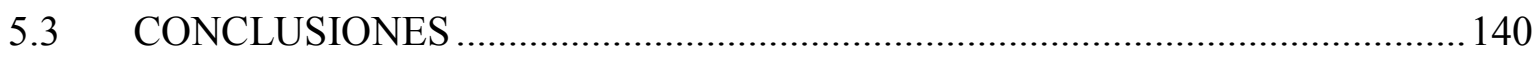

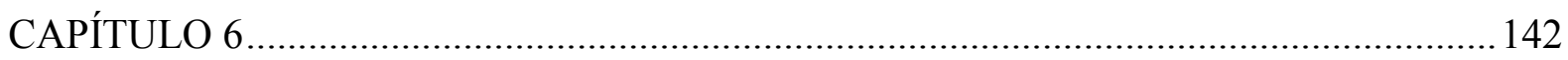

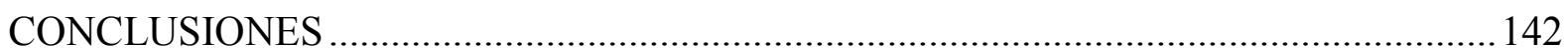

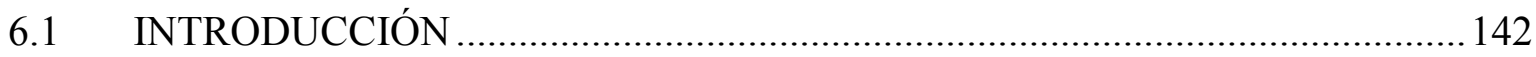

6.2 ESTUDIOS EXPERIMENTALES ............................................................... 142

6.3 ANÁLISIS DE RESULTADOS ...................................................................... 143

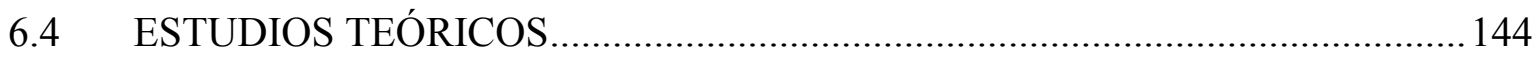




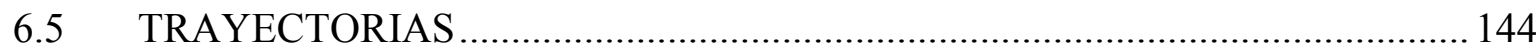

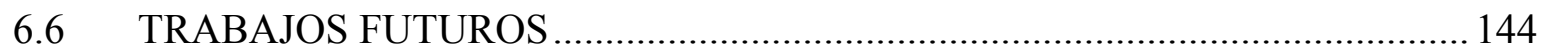

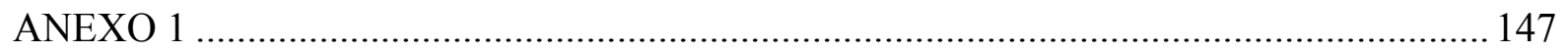

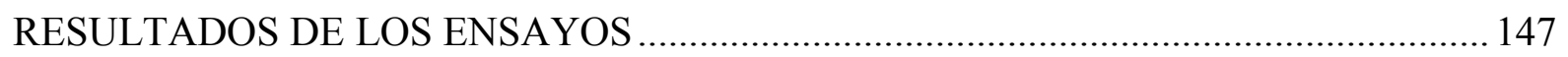

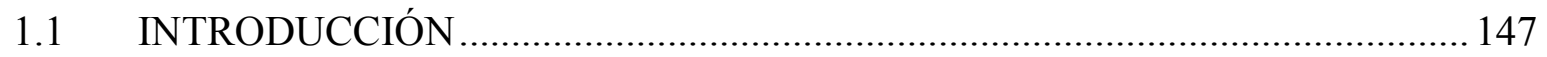

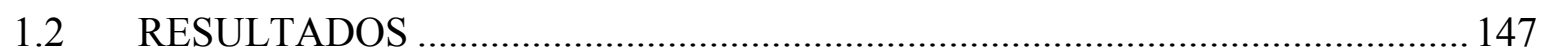

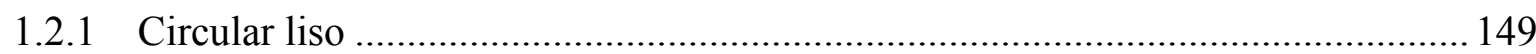

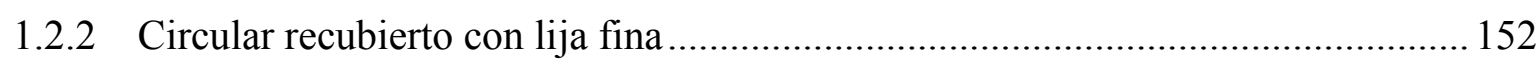

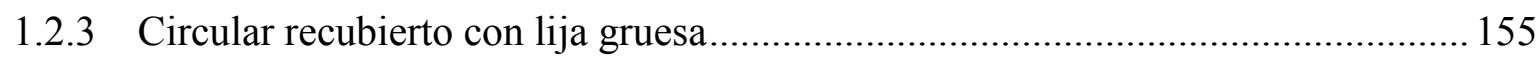

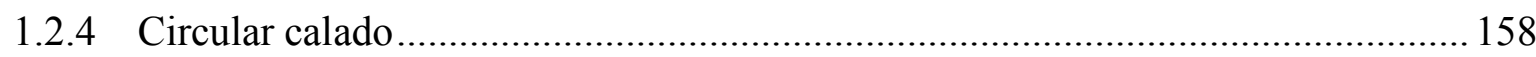

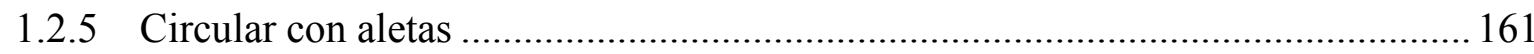

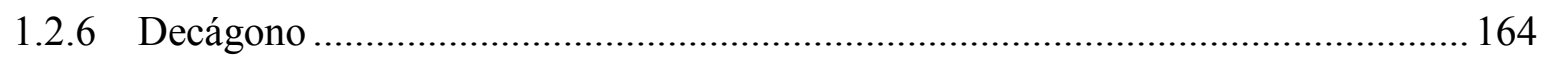

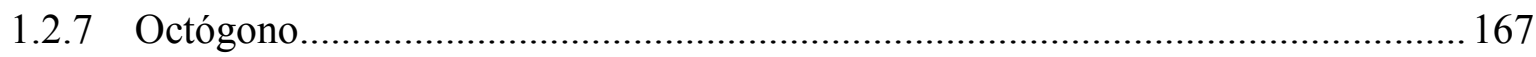

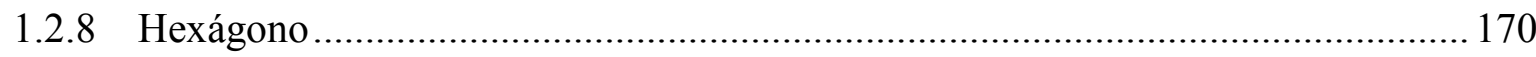

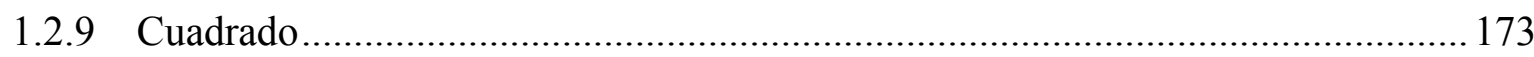

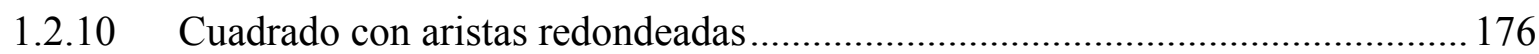

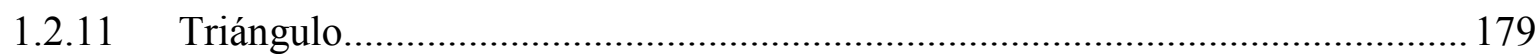

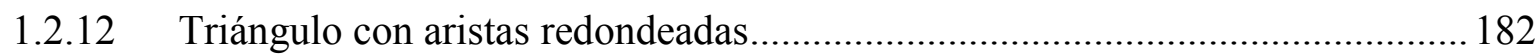

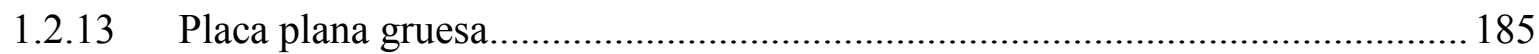

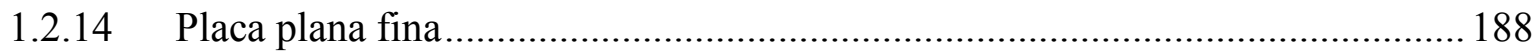

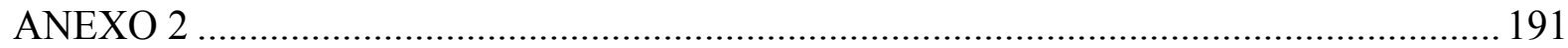


CALCULO DE INCERTIDUMBRES EN LAS MEDICIONES.

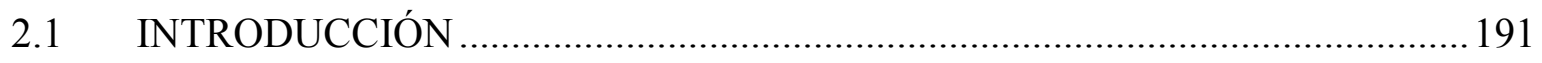

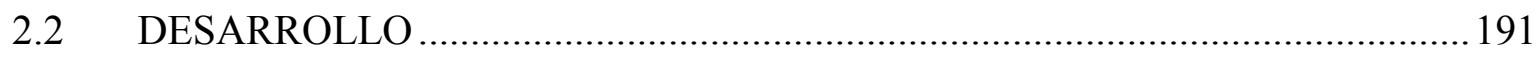

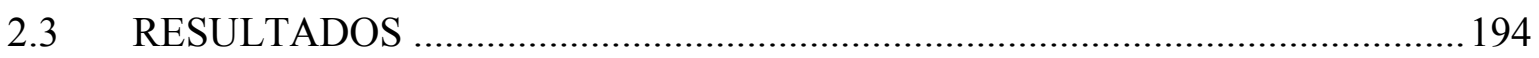

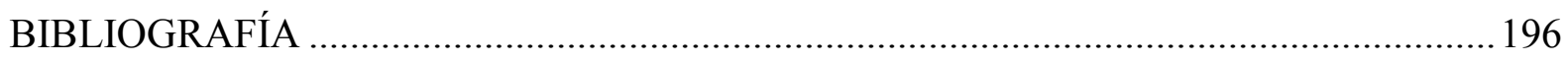




\section{NOMENCLATURA}

A

C

$C_{D}$

$C_{L}$

$C_{L A}$

$C_{M}$

$C_{M A}$

D

g

$h$

$\mathrm{h}_{\text {túnel }}$

$I$

$k$

$k \alpha$

$k \beta$

$l$

$L$

$m$
Ancho del túnel de viento

Área de la sección del túnel

Coeficiente de resistencia

Coeficiente de sustentación

Coeficiente de sustentación generado por la rotación

Coeficiente de momento

Coeficiente de momento generado por la rotación

Resistencia

Aceleración de la gravedad

Altura del modelo

Altura del túnel de viento

Momento de inercia del modelo

Parámetro de proporcionalidad para modelo teórico

Parámetro de proporcionalidad para modelo teórico

Parámetro de proporcionalidad para modelo teórico

Largo del modelo

Sustentación

Coeficiente teórico de bloqueo 
M

$n_{d}$

$n_{l}$

$n_{t}$

$q$

$R$

$\mathrm{R}_{\mathrm{e}}$

$S$

$\mathrm{S}_{\mathrm{t}}$

$t$

$t_{c}$

$t_{d}$

$t_{r}$

$t_{r l}$

$U$

$u$

$v$

$V_{c}$

$V_{\text {ref }}$

$v_{x}$

Vref $x$
Masa del modelo

Frecuencia de desprendimiento de torbellinos

Número de caras del cuerpo

Número de torbellinos por unidad de tiempo

Presión dinámica

Radio del modelo

Número de Reynolds

Superficie frontal del modelo

Número de Strouhal

Tiempo

Tiempo transcurrido entre paso de aristas del cuerpo

Tiempo de desprendimiento de torbellinos

Tiempo de recorrido de una partícula por el cuerpo

Tiempo de recorrido de una partícula por el cuerpo considerando un lado

Incertidumbre

Velocidad tangencial del modelo

Velocidad de la corriente libre

Velocidad corregida (estudio de bloqueo)

Velocidad de referencia (caracterización túnel)

Componente horizontal de la corriente libre

Velocidad en el eje $x$ (caracterización túnel) 
$V_{x} \quad$ Velocidad horizontal del modelo

$v_{y} \quad$ Componente vertical de la corriente libre

Vrefy Velocidad en el eje $y$ (caracterización túnel)

$V_{y} \quad$ Velocidad vertical del modelo

$x \quad$ Posición en eje $x$

$y \quad$ Posición en eje $y$

$\Gamma \quad$ Circulación

$\alpha \quad$ Ángulo de incidencia de la corriente libre

$\phi \quad$ Diámetro del modelo

$\mu \quad$ Viscosidad dinámica del aire

$\theta \quad$ Aceleración angular

$\rho \quad$ Densidad del aire

$v \quad$ Viscosidad cinemática del aire

$\omega \quad$ Velocidad angular, velocidad de giro, velocidad de rotación 


\section{RESUMEN}

El objeto de la presente tesis es el estudio experimental del efecto Magnus en diferentes cuerpos cilíndricos de secciones transversales diversas.

Surge del estudio de los antecedentes que el efecto Magnus es un fenómeno aerodinámico de gran importancia, y que ha sido estudiado desde distintas perspectivas, desde su aprovechamiento para propulsión de vehículos, dispositivos hipersustentadores en alas de aeronaves, proyectiles balísticos, deceleradores aerodinámicos, deportes donde se utilizan pelotas o bien, para el estudio de trayectorias de cuerpos en vuelo libre movidos por efecto del viento. No obstante es muy limitado el conocimiento que se tiene sobre el comportamiento de este efecto en cuerpos de sección transversal no circular, siendo esta la principal fuente de aporte al estado del arte de la presente tesis.

El efecto Magnus es un fenómeno aerodinámico que se da en cuerpos en rotación inmersos en una corriente de aire perpendicular al eje de rotación, en los cuales se genera una fuerza de sustentación perpendicular a dicha corriente, capaz de generar un cambio en la trayectoria de un cuerpo en vuelo libre.

El desarrollo de la presente tesis comprende la construcción de un túnel de viento para la realización de ensayos de medición de fuerzas y visualización de flujo, así como la fabricación de modelos a escala y la instrumentación necesaria para que a partir de los resultados obtenidos se pueda conocer el comportamiento aerodinámico de los diferentes cuerpos. Así mismo, se determinan los coeficientes aerodinámicos de cada cuerpo y se los clasifica en función de su respuesta a la variación de las velocidades de rotación y de la corriente libre.

A partir de conocer dichos resultados y las respuestas de cada cuerpo a las variaciones en las velocidades, se realiza un análisis de las posibles trayectorias que seguiría cada cuerpo para una condición de vuelo libre. 


\section{CAPÍTULO 1}

\section{INTRODUCCIÓN}

\subsection{INTRODUCCIÓN AL ESTUDIO EXPERIMENTAL DEL EFECTO MAGNUS}

Se denomina efecto Magnus al fenómeno físico en el cual un cuerpo en rotación inmerso en una corriente de aire experimenta una fuerza de sustentación, fuerza esta perpendicular al plano determinado por el eje de rotación y la dirección de la corriente libre del fluido; además de la fuerza de resistencia, en la dirección de la corriente libre. Dicho efecto fue llamado así por ser Heinrich Gustav Magnus (1802 - 1870), el primer investigador que ha estudiado este fenómeno en 1851.

Esta fuerza de sustentación o efecto Magnus puede tener un efecto importante en el movimiento de un cuerpo en rotación que se desplaza en un fluido. Es el causante de la desviación de la trayectoria de proyectiles que rotan sobre su eje longitudinal (para lograr mayor estabilidad), hecho que motivó los estudios de Magnus. También es posible observarlo en las trayectorias de pelotas de tenis, golf, fútbol y otros deportes.

Existen múltiples potenciales aplicaciones del estudio propuesto, como cohetes o lanzadores espaciales, proyectiles aéreos (antigranizo, bélicos, etc.), sondas atmosféricas, objetos arrastrados por el viento en tornados o vendavales, y su movimiento y trayectoria, el vuelo del balasto en las inmediaciones de las vías del tren de alta velocidad, la propulsión de buques, sistemas de hipersustentación en perfiles alares, etc. 


\subsection{ANTECEDENTES}

El presente trabajo aborda el estudio experimental del efecto Magnus para cuerpos no cilíndricos circulares (cuerpos de secciones transversales diversas). Dicho efecto ha sido estudiado en diferentes ámbitos, desde la náutica, la aeronáutica, el deporte, la meteorología, entre otros.

Seifert [1] realiza una publicación en la cual se presenta una compilación de los estudios del efecto Magnus a través de la historia, describiendo ensayos, aplicaciones en aeronáutica y náutica, y presentando ecuaciones y curvas útiles para la presente tesis.

A continuación se describen los antecedentes y la evolución de los estudios en el tiempo.

\subsubsection{Primeros estudios, cilindros de sección circular}

Los primeros estudios del efecto Magnus han sido con cilindros circulares. En 1925, Betz [2] publica en un reporte N.A.C.A un estudio relacionado con el "Rotor de Flettner", donde se describen los ensayos realizados sobre un cilindro en rotación inmerso en una corriente de aire (figuras $1.1 \mathrm{y} 1.2$ ).

a)

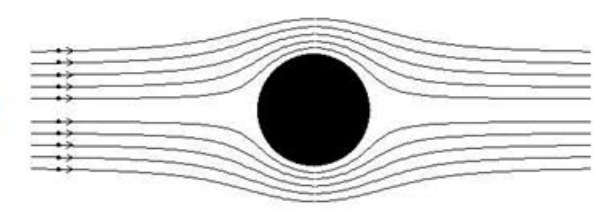

b)

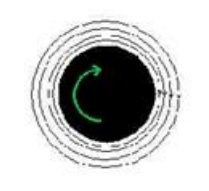

c)

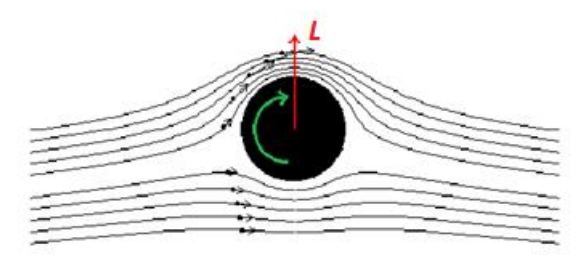

Figura 1.1 Efecto Magnus en un cilindro a) sin rotación en el seno de un flujo potencial, b) sólo con rotación y c) con ambas configuraciones y la generación de sustentación $(L)$. (estudio de Betz [2]). 
L. Prandtl en 1926 [3] amplia la anterior y realiza una publicación en la Revista "Die Naturwissenschaft" Vol. XIII, relacionada a la aplicación del efecto Magnus para la propulsión de barcos. En la misma hace un análisis de flujo potencial alrededor de un cilindro liso con circulación. Así mismo, la publicación presenta ensayos experimentales de visualización de flujo donde pueden observarse el flujo detrás de un cilindro en rotación sometido a una corriente de aire.

Esta publicación fue de gran utilidad para la presente tesis ya que en la misma se presentan resultados importantes de ensayos realizados por el autor donde se pueden determinar las variaciones de los coeficientes $C_{L}$ y $C_{D}$ en función de la velocidad de la corriente libre y de la de rotación de diferentes cilindros.

Por su parte Ahlborn [4] en 1929 estudia el efecto Magnus y realiza esquemas de flujo alrededor de un cilindro en rotación inmerso en una corriente de agua en reposo.

Se han hecho estudios para diferentes condiciones de flujo. Fletcher [5] ha realizado ensayos en un modelo de cilindro circular sometido a un régimen de flujo en condiciones de número de Reynolds crítico, donde se generan fuerzas de sustentación "negativas" (efecto Magnus negativo), de sentido opuesto al efecto Magnus "convencional", debidas a fenómenos asociados a la capa límite y al ángulo de inclinación del modelo respecto a la corriente. Así mismo, Muto [6] realiza un estudio similar del fenómeno de inversión del efecto Magnus para esferas sometidas a un flujo con número de Reynolds crítico.

Miller [7] en 1979 presenta una publicación basada en ensayos de un cilindro con vanos para generar autorrotación con la corriente libre. El mismo estaba equipado con una gran cantidad de tomas de presión a lo largo de su superficie y se determinaron los coeficientes de presión $C_{P}$ sobre el cuerpo en función de su posición y de las velocidades de giro y de la corriente. El estudio incluye una visualización de flujo alrededor del cuerpo, interesante para comprender los desprendimientos.

Leech [8] en 1980 estudia las ecuaciones de movimiento para un cilindro con seis grados de libertad y en el mismo contempla el efecto Magnus. Es un estudio interesante ya que se puede observar cómo influye dicho efecto en el movimiento de un cuerpo con rotación. 
Por otro lado, Howerton [9] realizó un estudio teórico y experimental de dos cilindros alineados con un mismo eje, con rotación, sometidos a una corriente de aire. Dicho sistema se ensayó a diferentes ángulos de inclinación.

Cuevas et al. [10] han realizado una publicación donde se calcula el efecto Magnus para un cilindro de manera teórica, utilizando un modelo de flujo potencial para calcular su expresión y se expone como se relaciona ésta con el desarrollo de la vorticidad para un cuerpo con circulación y sin circulación.

Además de los ensayos experimentales, muchos autores han realizado cálculos computacionales de cilindros en rotación mediante técnicas de CFD [11], donde se han determinado coeficientes aerodinámicos y visualizaciones de flujo alrededor y detrás de los cuerpos.

Así mismo Mittal y Kummar [12] han realizado un estudio numérico de CFD para un cilindro en rotación en el cual presentan diferentes patrones de flujo, incluyendo la estela para las distintas relaciones de velocidad angular y de la corriente libre.

Por otro lado, Gowree ha publicado en actas de un congreso [13] un trabajo relacionado con el cálculo numérico mediante CFD del efecto Magnus alrededor de un cilindro para altos números de Reynolds. En el mismo se analiza la variación de la capa límite y al igual que Fletcher, la inversión del efecto a altos números de Reynolds.

Ericcson [14] ha realizado un estudio del efecto que se produce en un cilindro circular en rotación y luego con eje de giro alineado a la corriente. Puede observarse un efecto tridimensional en el cual se produce un efecto de barrena plana al llegar al número de Reynolds crítico. Así mismo, ha analizado la influencia de variables como la rugosidad del cuerpo y diferentes velocidades de rotación.

Por su parte en [15], Ericcson ha realizado un estudio para calcular la respuesta, en base a conceptos de flujo no estacionario, de los desprendimientos de Kármán desde un cilindro circular en rotación en una corriente de aire. 


\subsubsection{Estudios en otros cuerpos}

Los estudios publicados en relación a cuerpos no romos en su mayoría son estáticos (sin rotación del modelo), pero han sido de gran utilidad para determinar coeficientes de sustentación y resistencia, y poder conocer los patrones de flujo detrás de los cuerpos. Entre ellos, Polhamus [16] realizó un estudio muy interesante en el cual se ensayaron muchos cuerpos cilíndricos no circulares, estáticos, para diferentes ángulos de incidencia de la corriente libre y para diferentes números de Reynolds. En el mismo se determinaron coeficientes de sustentación, resistencia y momento. Así mismo, se describen ensayos de los cilindros para diferentes ángulos de ataque.

Se han realizado estudios de cuerpos no cilíndricos circulares con aletas [17] sometidos a una corriente libre y se ha estudiado el efecto de autorrotación en función de la posición y geometría de dichas aletas. Dichos estudios experimentales han permitido determinar los patrones de flujo para diferentes ángulos de inclinación del cuerpo ensayado y la distribución de presiones alrededor del mismo. Se ha podido concluir acerca de las ventajas de cada modelo en términos de autorrotación. Así mismo, Skews [18] ha realizado ensayos de autorrotación de cilindros no circulares aislados y por otro lado, con un deflector corriente arriba en la que se ha estudiado la incidencia del mismo en los efectos de rotación del cuerpo. También ha determinado coeficientes aerodinámicos y relaciones entre las velocidades de rotación de los cuerpos y la inclinación del deflector.

Zaki [19] realizó un estudio numérico para un cuerpo rectangular bidimensional sometido a una corriente libre, para diferentes ángulos de inclinación. En el mismo determina los puntos de separación y los patrones de flujo para diferentes números de Reynolds y compara los resultados con un ensayo en un túnel de agua para visualizar el flujo y la estela producida.

Ozgoren [20] realizó un estudio experimental del patrón de flujo detrás de un cilindro circular y uno rectangular con dos inclinaciones respecto a la corriente libre. En el mismo presenta visualización de flujos para una variación determinada del número de Reynolds. Se pueden observar los desprendimientos alrededor de los cuerpos y la vorticidad en la estela.

Lysenko [21] ha realizado estudios de modelos de turbulencia de flujo separado mediante la utilización del software OpenFOAM en secciones circulares y triangulares sin rotación. 


\subsection{APLICACIONES DEL EFECTO MAGNUS}

Muchos estudios se han realizado en relación con aplicaciones directas del Efecto Magnus. A continuación se describen las más importantes.

\subsubsection{Proyectiles}

Las características del efecto Magnus sobre proyectiles, cohetes y lanzadores han sido muy estudiadas ya que en la mayoría de los casos los mismos son cuerpos cilíndricos de sección circular y su vuelo es de traslación con rotación en un medio de aire móvil, generando así la posibilidad de que por causas del efecto Magnus, existan variaciones en las trayectorias. Queijo, Fletcher y Herman [22] publicaron en 1957 un reporte en el cual se ensaya un cilindro de diferente sección, con rotación y con diferentes ángulos de inclinación respecto a la corriente. En el mismo se determinan coeficientes $C_{L}$ y $C_{D}$ para cada sección y cada ángulo de ataque. En esta publicación puede observarse la importancia del efecto Magnus para dispositivos tipo cohetes y proyectiles cilíndricos de sección variable.

Jacobson [23] en 1973 realiza un extenso estudio para la aplicación en proyectiles y balística, de cuerpos en rotación donde el efecto Magnus tiene una gran relevancia en la estabilidad del objeto. Se presentan ensayos en túnel de viento de modelos de cohetes con diferente geometría y que son para distintas utilidades. Así mismo, Platou [24] realiza un estudio de proyectiles balísticos con rotación sometidos a velocidades supersónicas.

Ericcson [25] en 1989 analiza el desprendimiento del flujo en un modelo de fuselaje cilíndrico circular de una aeronave a altos ángulos de ataque y el efecto que se produce con la rotación del mismo frente a la corriente. Dicho efecto produce desprendimiento asimétrico de la capa límite generando fuerzas laterales en el cuerpo.

Swanson, Iversen y Power [26] [27] [28] [29] [30] realizan una gran variedad de estudios teóricos y analíticos relacionados con misiles, estudiando diferentes ángulos de ataque y generando ecuaciones matemáticas que determinan los coeficientes de sustentación producidos por el efecto Magnus. Así mismo, Kelly [31] realiza un extenso estudio matemático para determinar la fuerza normal generada por la rotación de misiles a grandes ángulos de ataque. 
Todos los desarrollos teóricos realizados por estos autores son de suma importancia para la presente tesis. Muchos estudios, como son los de Beard [32], Milton [33] y Wang [34] se basaron en los previos ensayos y determinaciones de Kelly [31] para obtener resultados y determinar los coeficientes aerodinámicos en misiles y proyectiles balísticos.

\subsubsection{Vehículos}

Diversas aplicaciones se han estudiado en lo que respecta a vehículos, como se nombró en el punto 1.2.1 en barcos (figura 1.2), y en muchos caso en aeronaves, en el diseño de sistemas de hipersustentación.

Modi [35] en 1981 realiza un estudio de perfiles alares en los cuales el borde de ataque y el borde previo a la superficie móvil (alerón o flap) es reemplazado por cilindros con rotación. En el mismo se estudia la distribución de presiones a lo largo del ala, para diferentes ángulos de ataque, diferentes velocidades de rotación de los cilindros y diferentes ángulos de inclinación de la superficie móvil. Es interesante este estudio porque se pueden observar las variaciones de sustentación gracias a los cilindros en rotación y el análisis de entrada en pérdida a grandes ángulos de ataque.

Ericcson [36] en 1995 analiza de manera analítica y experimental la separación y readherencia del flujo en alas de diferentes modelos mediante la utilización de perfiles con bordes de ataque cilíndricos con rotación.

Al Garni publica en el 2000 un estudio experimental [37] en el cual se reemplaza el borde de ataque fijo de un modelo de ala por un cilindro con rotación y se lo ensaya en el túnel de viento para diferentes ángulos de ataque, posición de flaps, velocidad de la corriente y velocidad de giro del cilindro. Determina coeficientes aerodinámicos para cada condición y caracteriza aerodinámicamente el modelo. Asimismo estudia las variaciones del tipo de capa límite y desprendimientos mediante visualizaciones de flujo con hilos de humo.

Kano y Yagita [38] en el año 2002 realizaron ensayos experimentales de un cilindro en rotación con el piso del túnel con movimiento, para poder estudiar el efecto suelo que se genera en la cercanía del cilindro. En el mismo se midieron presiones, velocidades y se realizaron visualizaciones de flujo, donde se pudieron observar patrones tridimensionales. Se determinaron coeficientes aerodinámicos del cilindro. 
A modo informativo, se puede nombrar que a principios de los 80 , se diseñó un vehículo de transporte de carga mediante un globo esférico equipado con aletas y motores que se desplazaba mediante la rotación del mismo. Se presentó una publicación [39] en el Journal of Aircraft donde se describen algunas de las características aerodinámicas de la esfera en rotación inmersa en una corriente de aire.

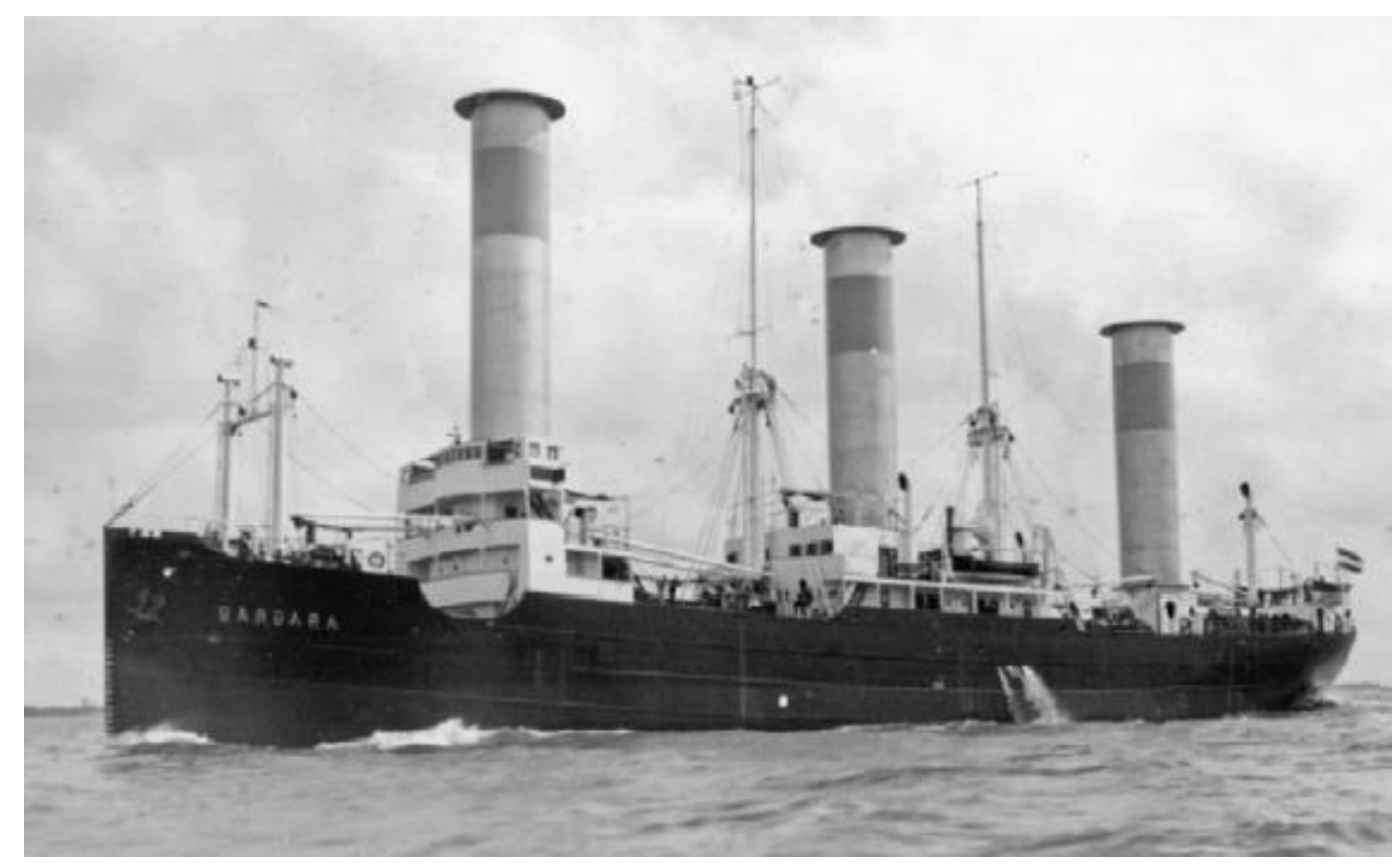

Figura 1.2 Fotografía de un barco propulsado por tres cilindros en rotación [1].

\subsubsection{Deportes}

Se han realizado muchos estudios relacionados con los deportes y el movimiento de pelotas en el aire. En el caso de Bahill [40] se describe el caso de una pelota de baseball arrojada con velocidad angular y de traslación, y los efectos aerodinámicos que en esta se generan a causa de las condiciones atmosféricas y la altura. Es importante ver como las variaciones de presión, densidad y humedad generan cambios considerables en el movimiento de la bola.

Así mismo, Leroy y Hubbard [41] realizaron un estudio del vuelo de la pelota de baseball a partir de la rotación de la misma, considerando todas las fuerzas que están presentes sobre el cuerpo. Generaron visualizaciones con cámaras de alta velocidad y determinaron trayectorias. Además realizaron un análisis matemático aplicando ecuaciones de movimiento. 
Barber [42] realizó un estudio numérico computacional en CFD donde analiza diferentes tipos de pelotas de fútbol y las clasifica según su terminación superficial. En el mismo analiza patrones de flujo para diferentes velocidades de rotación y traslación. Determina coeficientes aerodinámicos y analiza trayectorias.

Un importante estudio es el realizado en pelotas de golf [43] en el cual se hace un análisis fluidodinámico en CFD teniendo en cuenta además de las velocidades de rotación y traslación, el efecto de la rugosidad de la superficie y las variaciones en las trayectorias que esto genera.

Por otro lado, Kray [44] ha realizado un estudio teórico, numérico y experimental de una esfera en rotación para altos números de Reynolds, donde se analizan los puntos de separación y los patrones de flujo alrededor de la esfera.

\subsubsection{Objetos en vuelo libre}

Dicho estudio es para objetos en vuelo libre con rotación, en los cuales, por causas del efecto Magnus sus trayectorias pueden variar en función de las velocidades de rotación y translación.

Minami y Azuma han publicado [45] estudios en los que se analiza el vuelo libre de sámaras y otras especies de semillas que caen de los árboles en autorrotación. Lo interesante de estos estudios es el análisis aerodinámico que se realiza en cuerpos que giran.

Existen estudios en los cuales se tiene en cuenta el efecto para el desplazamiento de objetos como ser piedras. Nguyen publica un estudio [46] en el cual se estudia el desplazamiento de una piedra expulsada por el contacto con una rueda de una aeronave en movimiento.

Por otro lado, Holmes [47] ha publicado un interesante estudio en el cual se analizan trayectorias de vuelo de esferas, como modelos de simulación de objetos dispersos en las inmediaciones de un tornado. En el mismo analiza efectos de la resistencia en tres dimensiones y efectos generados por la turbulencia.

Para los análisis de trayectorias, se representan en las publicaciones [48] y [49] ensayos experimentales en túnel de viento en los cuales se puede observar el comportamiento de una placa plana liberada en una corriente de viento; y el estudio de dichos ensayos de manera analítica, donde se pueden determinar los patrones de vuelo. Dentro de las consideraciones se 
tienen en cuenta los efectos de la rotación y la sustentación generada por el efecto Magnus y el desvío que este genera en la trayectoria del objeto.

Referido a esta temática, Baker [50] en 2007 publica un análisis matemático de las ecuaciones de las trayectorias, en dos dimensiones, del vuelo de objetos movidos por fuertes vientos. En el mismo tiene en cuenta la rotación de los objetos, la resistencia y la sustentación generada por las corrientes de aire. Dichas ecuaciones tienen en cuenta las condiciones atmosféricas para el caso del análisis del vuelo de objetos en tornados o tormentas de viento.

Esta publicación ha sido de suma importancia para la presente tesis ya que con ayuda de dichas ecuaciones se ha podido determinar la trayectoria de los cuerpos ensayados.

En estudios relacionados con el vuelo de objetos ante fuertes vientos, como es el caso de chapas de techos [51], se puede observar que al ser cuerpos planos en vuelo libre y con autorrotación, se tiene en cuenta el efecto Magnus para la variación de la trayectoria. En dicha publicación se hacen análisis de diferentes geometrías de chapas para determinar los patrones de vuelo.

Richards [52] publicó estudios numéricos para el cálculo de las trayectorias en tres dimensiones del vuelo de objetos desplazados por fuertes vientos.

Kakimpa, Hargreaves y Owen [53] [54] [55] realizaron estudios en dos y tres dimensiones del vuelo de una chapa con rotación mediante técnicas experimentales (figura 1.3) y computacionales de CFD. Así mismo, Hirata [56] realizó un análisis completo y caracterizó aerodinámicamente chapas en vuelo libre.

Kordi [57] ha presentado un estudio acerca de modelos de configuraciones de techos de casa con el objeto de analizar el comportamiento y trayectoria de chapas que se vuelan por vientos fuertes. Determinan que el vuelo posee rotación en los tres ejes y que se ve muy afectado por la sustentación generada por el efecto Magnus.

Así mismo Maruyama [58] ha generado modelos numéricos de simulación para ver el comportamiento del vuelo de objetos producido por tornados con patrón circular. 

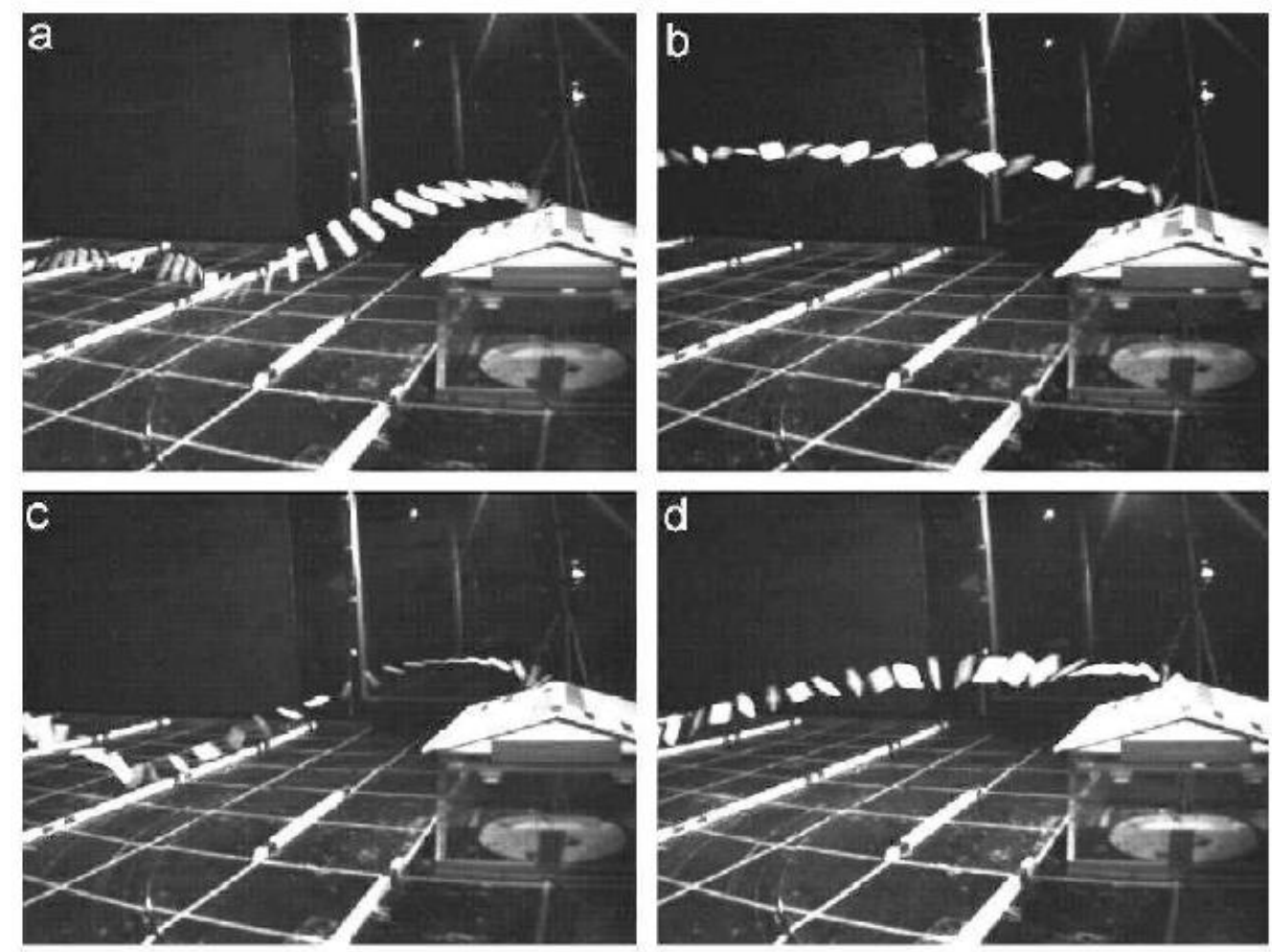

Figura 1.3 Estudio de la traslación y rotación de chapas de techos que se vuelan por efecto del viento (estudio de Kakimpa et al.).

Se presenta en la tabla 1.1 el resumen de las referencias mencionadas:

Tabla 1.1 Resumen de referencias

\begin{tabular}{|c|c|c|c|}
\hline Tipos de cuerpo & Movimiento & Desarrollo & Ref. \\
\hline Cilindro circular & Rotación & Experimental & {$[2][4][9]$} \\
\hline Cilindro circular & Rotación & $\begin{array}{c}\text { Experimental } \\
\text { Teórico }\end{array}$ & {$[3][5][14][15]$} \\
\hline Esfera & Rotación & CFD * & {$[6]$} \\
\hline Cilindro con aletas & Autorrotación & Experimental & {$[7]$} \\
\hline Cilindro circular & Rotación & Teórico & {$[8][10]$} \\
\hline Cilindro circular & Rotación & CFD & {$[11][12][13][21]$} \\
\hline $\begin{array}{c}\text { Cilindro rectangular } \\
\text { Cilindro triangular } \\
\text { Cilindro cuadrado }\end{array}$ & Estático & Experimental & {$[16]$} \\
\hline Cilindro rectangular & Autorrotación & Experimental & {$[17]$} \\
\hline Cilindros no circulares & Autorrotación & Experimental & {$[18]$} \\
\hline Cilindro rectangular & Rotación & CFD & {$[19]$} \\
\hline $\begin{array}{c}\text { Cilindro circular } \\
\text { Cilindro rectangular }\end{array}$ & Rotación & CFD & {$[20]$} \\
\hline
\end{tabular}




\begin{tabular}{|c|c|c|c|}
\hline Tipos de cuerpo & Movimiento & Desarrollo & Ref. \\
\hline $\begin{array}{l}\text { Proyectil circular } \\
\text { sección variable }\end{array}$ & Rotación & Experimental & {$[22]$} \\
\hline Proyectiles & Rotación & Experimental & {$[23]$} \\
\hline $\begin{array}{c}\text { Proyectiles } \\
\text { Régimen supersónico }\end{array}$ & Rotación & Experimental & {$[24]$} \\
\hline Fuselaje cilíndrico & Rotación & $\begin{array}{l}\text { Experimental } \\
\text { Teórico }\end{array}$ & {$[25]$} \\
\hline Proyectiles & Rotación & Teórico & $\begin{array}{c}{[26][27][28][29]} \\
{[30][31]} \\
\end{array}$ \\
\hline Proyectiles & Rotación & $\begin{array}{l}\text { Experimental } \\
\text { Teórico }\end{array}$ & [32] [33] [34] \\
\hline $\begin{array}{l}\text { Cilindro circular } \\
\text { perfiles alares }\end{array}$ & Rotación & $\begin{array}{l}\text { Experimental } \\
\text { Teórico }\end{array}$ & {$[35][36][37]$} \\
\hline $\begin{array}{l}\text { Cilindro circular } \\
\text { Efecto suelo }\end{array}$ & Rotación & Experimental & {$[38]$} \\
\hline $\begin{array}{l}\text { Esfera en rotación } \\
\text { Globo aerostático }\end{array}$ & $\begin{array}{l}\text { Rotación } \\
\text { Traslación }\end{array}$ & Experimental & [39] \\
\hline $\begin{array}{c}\text { Esfera } \\
\text { Pelota de baseball }\end{array}$ & $\begin{array}{l}\text { Rotación } \\
\text { Traslación }\end{array}$ & $\begin{array}{l}\text { Experimental } \\
\text { Teórico }\end{array}$ & {$[40][41]$} \\
\hline $\begin{array}{c}\text { Esfera } \\
\text { Pelota de futbol y de golf }\end{array}$ & Rotación & CFD & {$[42][43]$} \\
\hline Esfera & Rotación & $\begin{array}{l}\text { Experimental } \\
\text { Teórico }\end{array}$ & {$[44]$} \\
\hline Sámara & $\begin{array}{l}\text { Rotación } \\
\text { Traslación }\end{array}$ & $\begin{array}{l}\text { Experimental } \\
\text { Teórico }\end{array}$ & {$[45]$} \\
\hline Objetos varios & $\begin{array}{l}\text { Rotación } \\
\text { Traslación }\end{array}$ & Teórico & {$[46][47]$} \\
\hline Placas planas & Autorrotación & $\begin{array}{l}\text { Experimental } \\
\text { Teórico }\end{array}$ & [48] [49] \\
\hline Objetos varios & $\begin{array}{l}\text { Rotación } \\
\text { Traslación }\end{array}$ & Teórico & {$[50]$} \\
\hline Chapas de techos & $\begin{array}{l}\text { Rotación } \\
\text { Traslación }\end{array}$ & $\begin{array}{l}\text { Experimental } \\
\text { Teórico } \\
\end{array}$ & $\begin{array}{c}{[51][52][53][54]} \\
{[55][56][57]} \\
\end{array}$ \\
\hline Objetos varios & Rotación & Teórico & {$[58]$} \\
\hline
\end{tabular}

*CFD: Acrónimo de Computational Fluid Dynamics (Dinámica de Fluidos Computacional). 


\subsection{OBJETIVO DE LA TESIS}

En la presente tesis se pretende determinar de manera experimental las fuerzas aerodinámicas en cuerpos cilíndricos de secciones transversales diversas en rotación, inmersos en una corriente de aire, cuando el eje de rotación es perpendicular a la dirección de la corriente libre. De esta manera se caracterizarán aerodinámicamente 14 cuerpos, dentro de los cuales, en su mayoría, no se han encontrado en la literatura ensayos de este tipo, por lo que se considera un aporte novedoso de la presente tesis. Los cuerpos a ensayar son de diferentes secciones transversales, de diferente terminación superficial, cuerpos con bordes redondeados, cuerpos ranurados, con aletas, entre otros.

Se determinarán las fuerzas de sustentación (Magnus) y resistencia, además de los coeficientes de sustentación y resistencia para diferentes velocidades de rotación de los cuerpos en un rango de velocidades de la corriente de aire. Cabe destacar que la rotación de los cuerpos se realizará aplicando un par exterior y no por el fenómeno de autorrotación, en el cual la velocidad de rotación es dependiente de la velocidad de la corriente libre. De esta forma se recogerá información para modelizar el comportamiento en rotación del cuerpo fuera de la situación de equilibrio en autorrotación.

Así mismo, se realizarán visualizaciones de la configuración fluidodinámica en el entorno de los cuerpos a ensayar para las diferentes condiciones de ensayo antes mencionadas. En la literatura se encuentran estudios de visualización de flujo sobre cuerpos de sección circular ([3] [4] [7] [20] [37] [38] [41]), pero no sobre cuerpos con las formas de los mencionados para la presente tesis, por lo que se considera las visualizaciones un aporte original.

Se pretende encontrar generalizaciones sobre la influencia de la geometría del cuerpo en las fuerzas aerodinámicas.

El conocimiento de las fuerzas aerodinámicas generadas por los cuerpos estudiados y sus coeficientes aerodinámicos permitirá determinar las trayectorias en vuelo de cuerpos con estas secciones transversales, por lo que una aplicación de los resultados será la predicción de trayectorias de cuerpos en rotación movidos por el viento. Lo cual es aplicable para minimizar daños a personas e instalaciones, en caso de escombros que vuelan en una tormenta, tornados o bien, inmediaciones de vías de tren o autopistas, entre otras. 


\subsection{CONTENIDO DE LA TESIS}

La tesis se encuentra ordenada por capítulos.

El capítulo 1 se inicia con una introducción al efecto Magnus donde se describen los principios físicos por los cuales este se origina. En esta introducción se explica por qué se desarrollan ensayos experimentales y los fenómenos que aparecen en ensayos de este tipo. A su vez en dicho capítulo se realiza una revisión del estado del arte, en el que se recopila la información estudiada relacionada con esta temática en particular. Así mismo se detallan posibles aplicaciones de sistemas que utilizan este principio.

El Capítulo 2 comienza con una descripción de los ensayos iniciales que se han realizado con el fin de validar las metodologías de ensayo para los modelos utilizados en la presente tesis. Luego se presenta una descripción detallada del banco de ensayo que se desarrolló para todos los ensayos de medición de fuerzas aerodinámicas de cada modelo y la caracterización del mismo. Se describen todos los componentes e instrumentos utilizados para tales mediciones. Por otro lado, se presenta una descripción detallada (materiales utilizados y geometría) de cada modelo utilizado.

En el mismo capítulo se definen los dos tipos de ensayos que se realizaron, uno de medición de fuerzas y otro de visualizaciones. Se mencionan los procedimientos de ensayo y se presentan ejemplos de los resultados. Se presentan conclusiones de los ensayos. Por otra parte, se realiza un estudio teórico y experimental del bloqueo que aparece por utilizar un túnel de viento que ocupa el $15,75 \%$ de la sección transversal del túnel. Se determinan correcciones y se concluye al respecto.

En el Capítulo 3 se realiza un análisis de cada cuerpo a partir de los resultados obtenidos en los ensayos detallados en el Capítulo 2. Se clasifican los cuerpos en familias en función de la respuesta aerodinámica a la variación de velocidades, y se analiza su comportamiento. Se realiza un estudio del flujo alrededor de los cuerpos a partir de las fotografías obtenidas en los ensayos de visualización y se concluye.

En el Capítulo 4 se realiza un estudio de las publicaciones que presentan desarrollos teóricos del efecto Magnus. Se mencionan dichos desarrollos y, a partir de los mismos, se pretende encontrar una solución teórica a los ensayos de la presente tesis. 
Se concluye que ninguna de las publicaciones estudiadas desarrolla ecuaciones que ajusten al tipo de ensayo experimental de la tesis y por tal se realiza un desarrollo analítico para obtener ecuaciones teóricas que puedan determinar el comportamiento aerodinámico de los cuerpos ensayados. Se obtiene un algoritmo de ajuste que permite representar la sustentación de los cuerpos a partir de la relación de velocidades $u / v$ y el número de caras de los mismos (siempre mayor a 4 caras).

En el Capítulo 5 se realiza un estudio de posibles trayectorias en autorrotación de alguno de los cuerpos ensayados a partir de ecuaciones de movimiento publicadas por Baker [50]. Se plantean hipótesis, se calculan trayectorias y se concluye.

En el Capítulo 6 se presentan las conclusiones del estudio realizado en la presente tesis y las posibles líneas de estudio a futuro.

En el Anexo 1 se presentan los resultados de los ensayos realizados para la presente tesis. Se presentan tablas de resultados y gráficos de $L$ vs $v, C_{L}$ vs $u / v, D$ vs $v$ y $C_{D}$ vs $u / v$, para cada condición de velocidad de rotación y de la corriente libre, para cada cuerpo.

En el Anexo 2 se presenta el cálculo de la incertidumbre para la obtención de los coeficientes aerodinámicos $C_{L}$ y $C_{D}$, y la relación de velocidades $u / v$. Se estudian las fuentes de incertidumbre y se presentan las ecuaciones utilizadas para dichos cálculos. Se presentan los resultados y se concluye.

Finalmente se presenta la bibliografía de referencia de la presente tesis. 


\section{CAPÍTULO 2}

\section{ESTUDIOS EXPERIMENTALES}

\subsection{INTRODUCCIÓN}

Los primeros ensayos experimentales se planificaron para ser realizados en el túnel de viento cerrado del Departamento de Aeronáutica de la Facultad de Ingeniería de la UNLP. Al ser este de grandes dimensiones implicaba que los modelos también tengan que serlo generando problemas en su fabricación. Por tal, se diseñó, se instrumentó y se calibró un nuevo túnel aerodinámico para la realización de ensayos de medición de sustentación y resistencia en distintos modelos de cuerpos a diferentes velocidades de rotación y diferentes velocidades de la corriente de aire. Así mismo, se lo equipó con el instrumental necesario para la realización de visualizaciones de flujo. Se obtuvieron resultados y se concluyó acerca de los mismos.

\subsection{ENSAYOS INICIALES}

A modo de una aproximación y verificación del método de ensayo, se realizó un primer ensayo de un cilindro circular en rotación dentro del túnel de viento. Se midieron fuerzas de sustentación y resistencia, y parámetros de ensayo. Se utilizó el túnel de viento del Departamento de Aeronáutica de la Facultad de Ingeniería de la UNLP. El mismo es un túnel cerrado con una sección de pruebas rectangular de $1 \mathrm{~m}$ x 1,4 m y con capacidad de variar la velocidad de la corriente libre mediante la utilización de un variador.

El cilindro utilizado es de aluminio, con un largo de $760 \mathrm{~mm}$ y un diámetro de $76 \mathrm{~mm}$, posee dos tapas en sus extremos (figura 2.1), una con un acople para colocar el eje del motor y la otra posee en su interior un rodamiento con un eje, de modo que pueda sujetarse la balanza y no transmitirle movimiento de rotación a la misma. 
Cabe destacar que el eje es de sección circular y posee dos caras paralelas planas para hacer contacto con las guías del soporte de la balanza.

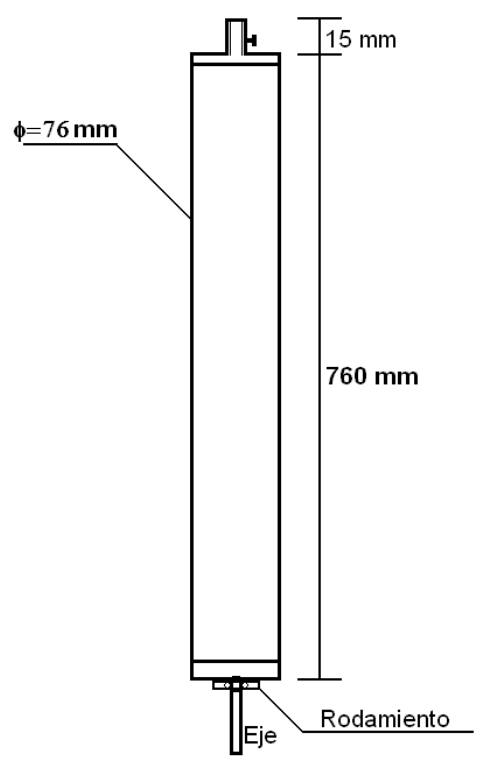

Figura 3.1 Primer modelo de cilindro de aluminio.

El motor utilizado es de corriente alterna de $220 \mathrm{~V}$ y entrega un máximo de $2800 \mathrm{rpm}$. En el mismo, se sujeta la articulación, mediante un eje. La articulación posee dos rodamientos y se acopla directamente a un marco ubicado en el exterior sobre el techo de túnel de viento (figura 2.2).
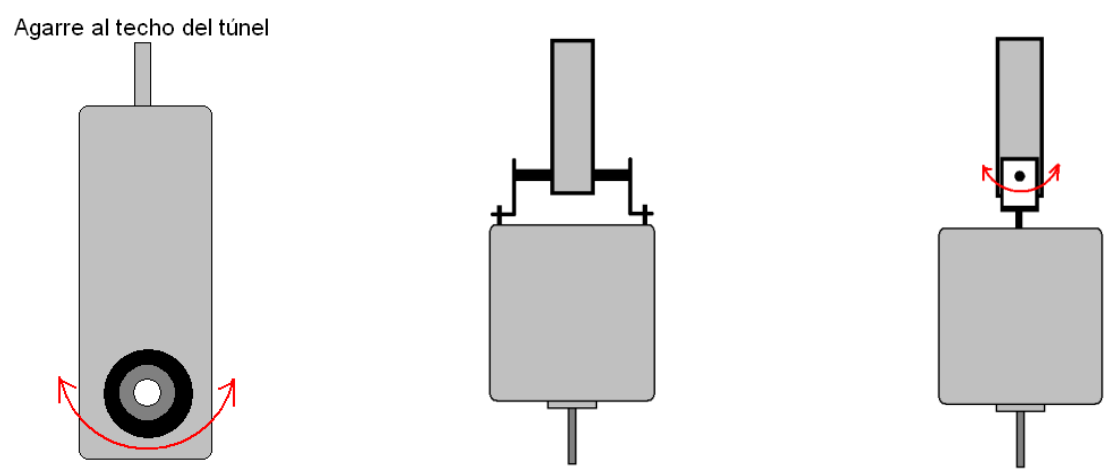

Figura 3.2 Sistema de articulación y motor pendulante. 
El soporte para la balanza posee dos guías de modo que se pueda contener el eje inferior del modelo y hacer que este se mueva sólo en un sentido. Las guías van fijadas al piso del túnel de viento.

El soporte se puede girar $90^{\circ}$ de modo que se pueda medir tanto la fuerza de sustentación como la de resistencia (figura 2.3).

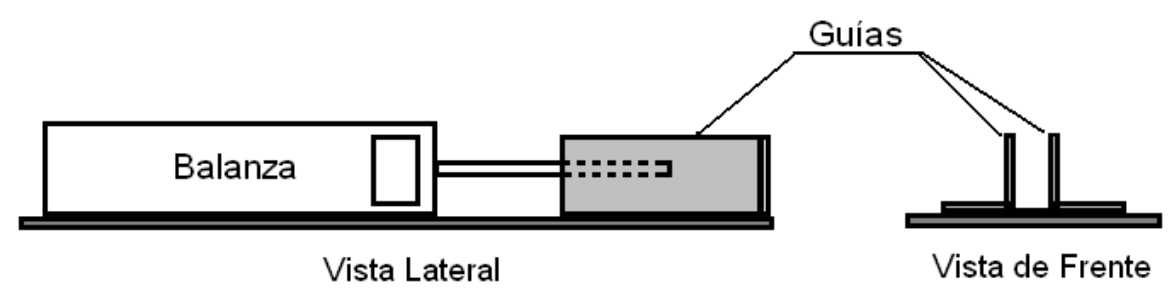

Figura 3.3 Sistema de guías y balanza para medición de fuerzas.

El modelo completo puede visualizarse en el esquema de la figura 2.4 y en la fotografía de la figura 2.5 .

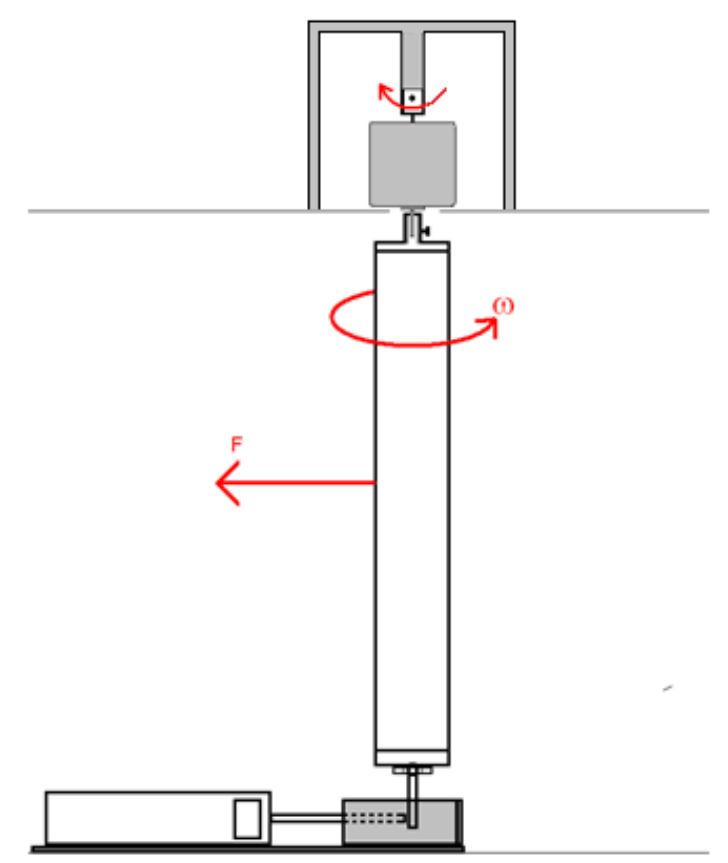

Figura 3.4 Sistema completo montado en el túnel de viento. 
Cabe destacar que la velocidad de rotación se midió con un tacómetro estroboscópico, la velocidad de la corriente libre con un tubo Pitot y un micromanómetro de columna de alcohol, y se reguló la velocidad de giro del motor con un variador de tensión.

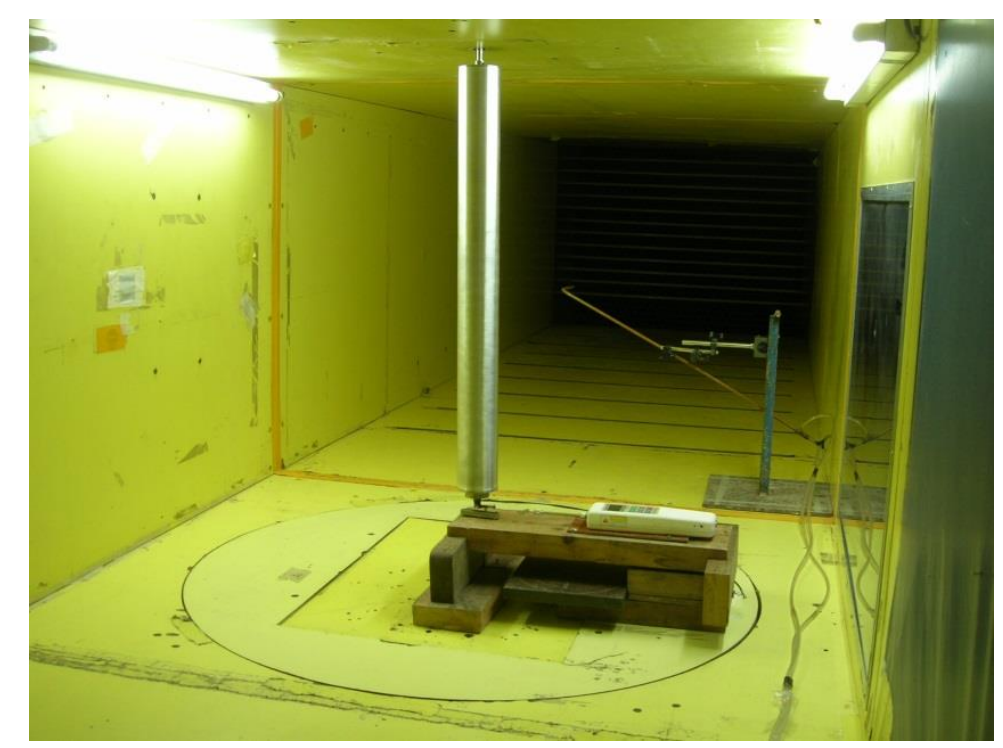

Figura 3.5 Sistema completo montado en el túnel de viento.

Este ensayo sirvió para determinar que este tipo de ensayo es posible y pueden determinarse resultados importantes. Pero el mismo fue descartado ya que por las grandes dimensiones de los modelos se hacía muy dificultosa la fabricación y el balanceo de los mismos.

Por tales motivos se diseñó y construyó un túnel de viento nuevo con el fin de ensayar modelos de menor escala, de más fácil y económica construcción y operación.

\subsection{BANCO DE ENSAYO Y EQUIPAMIENTO}

Siguiendo las indicaciones de la Norma IRAM 19004 se construyó un túnel de viento para realizar ensayos de medición de sustentación y resistencia en función de la velocidad de rotación para diferentes cuerpos cilíndricos. El mismo consta de las siguientes secciones y componentes:

Sección de Prueba: El túnel tiene una sección transversal de 0,2 x 0,2 m: la sección de prueba tiene una longitud de $0,3 \mathrm{~m}$, donde se monta el modelo a ensayar. Dicha sección está provista 
de una ventana de acrílico con el fin de observar el ensayo y permitir la medición de la velocidad de rotación de los modelos desde el exterior mediante el uso de un tacómetro estroboscópico. Además, tiene una tapa superior para montar los modelos con comodidad. Cabe aclarar que la misma se reemplaza por una de vidrio con el fin de realizar ensayos de visualización de flujo.

Así mismo en el exterior posee de una bancada móvil para el montaje del conjunto balanzamotor.

Sección de medición de presiones: En dicha sección se encuentra montada una toma de presión total en el centro y cuatro tomas de presión estática ubicadas en cada pared. Las tomas de presión estática se encuentran conectadas entre sí y tienen una sola salida al micromanómetro. Midiendo la presión total y la presión estática se puede calcular la presión dinámica y así obtener la velocidad de la corriente.

En la figura 2.6 se puede observar la sección de prueba y la sección de medición de presiones.

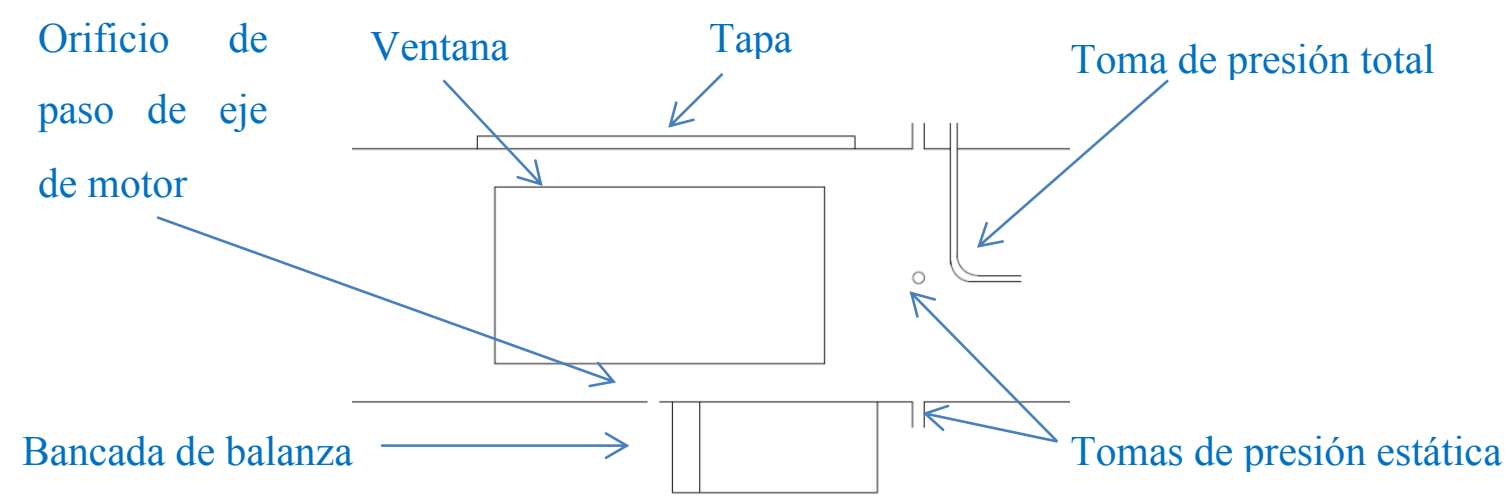

Figura 3.6 Sección de prueba y sección de medición de presiones.

Sección de enderezadores de flujo: Los mismos están construidos de caños de PVC de $2 \mathrm{~cm}$ de diámetro y poseen un largo de $10 \mathrm{~cm}$. Se encuentran pegados entre sí y cubren toda la sección del túnel.

Difusor: Cumple la función de adaptar la salida del soplador al túnel. Tiene $350 \mathrm{~mm}$ de largo y genera una contracción del 50\% de la sección del túnel. 
Bancada de balanza: Tiene la función de soportar la balanza. Tiene la posibilidad de rotarse $90^{\circ}$ con el fin de poder medir sustentación y resistencia en mismo paso.

Soplador: Es del tipo centrífugo con un caudal nominal de $7.000 \mathrm{~m}^{3} / \mathrm{h}$. El mismo posee un motor trifásico Siemens Schuckert de 3 HP. Para obtener diferentes caudales se utiliza un variador de frecuencia que se encarga de regular la velocidad de rotación del motor.

Variador de frecuencia: Es de uso comercial marca ABB modelo ACS150. Se utiliza para poder variar la velocidad de rotación del motor del soplador y así, seleccionar el caudal deseado para el ensayo.

A continuación se detalla un gráfico (figura 2.7) acotado con cada uno de los componentes del túnel de viento.

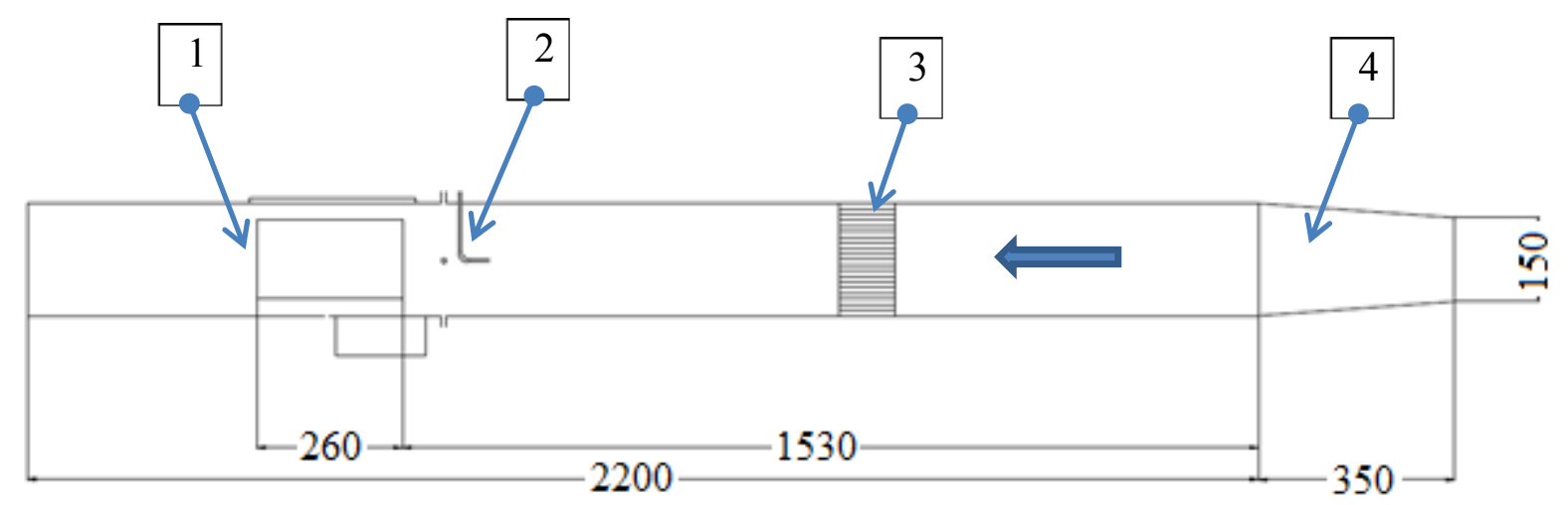

Figura 3.7 Túnel completo (unidades en mm). Código de identificación en tabla 2.1.

En la tabla 2.1 se explican los códigos de identificación de las referencias de la figura 2.7.

Tabla 2.1 Referencias.

\begin{tabular}{|c|c|}
\hline Referencia & Componente \\
\hline 1 & Sección de pruebas \\
\hline 2 & Sección de medición de presiones \\
\hline 3 & Enderezadores de flujo \\
\hline 4 & Difusor \\
\hline
\end{tabular}

El modelo completo se puede visualizar en la fotografía de la figura 2.8. 


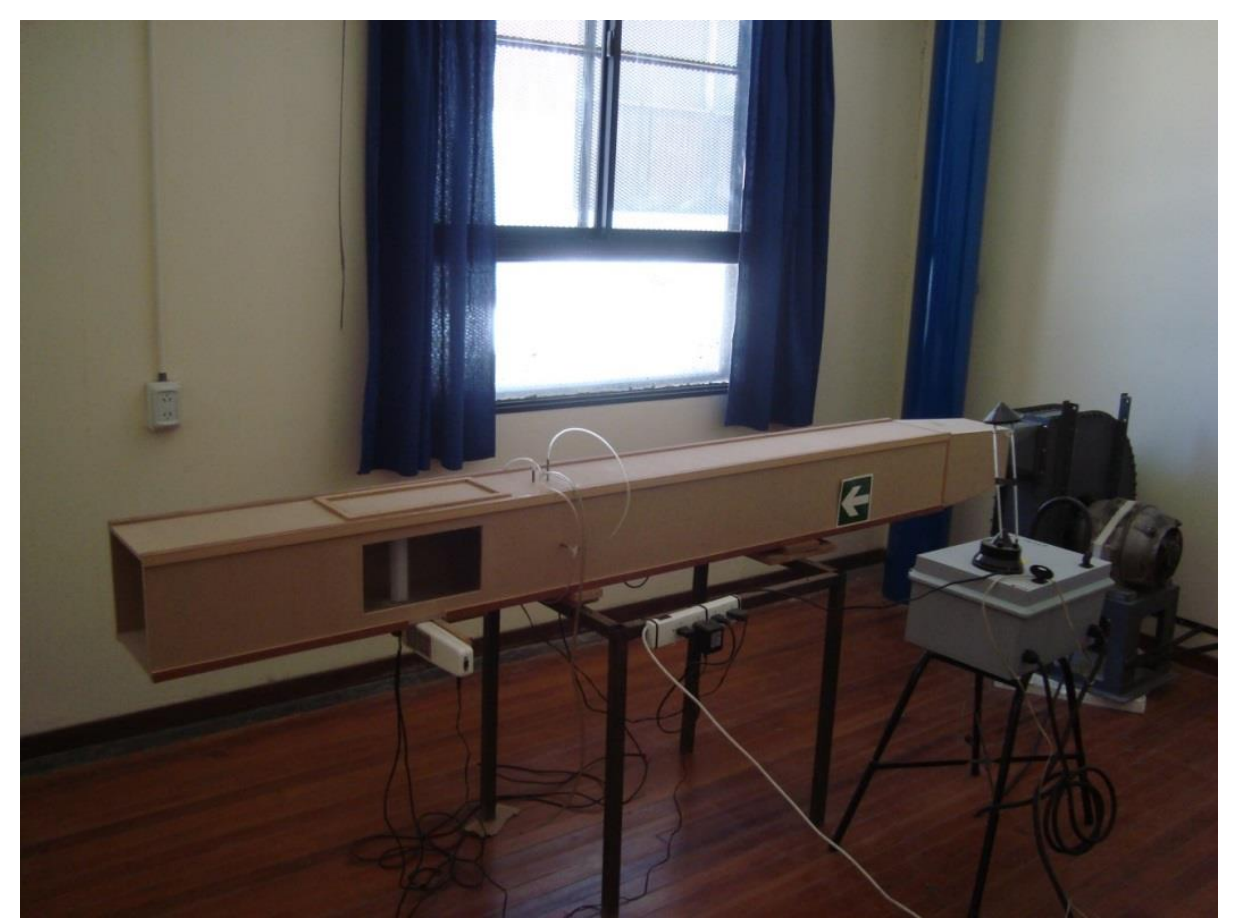

Figura 3.8 Fotografía túnel completo.

\subsubsection{Equipamiento}

Para la realización completa de los ensayos se utilizó el siguiente equipamiento:

Micromanómetro: Micromanómetro diferencial de columna inclinada de alcohol marca RFuess. El mismo se conecta a las dos tomas de presión, la estática y la total; de este modo se puede conocer la presión dinámica de manera directa.

Rango: 0 - 250 mm Col. Agua. Resolución: 0,05 mm Col. Agua.

Balanza: Balanza digital marca Schwyz modelo DINDIG10N. La misma se encuentra montada sobre la bancada ubicada en el piso del túnel del lado exterior. Sobre el sensor de la balanza se encuentra montado el motor que permite el giro de los modelos.

Rango: 0 - 10 N. Resolución: 0,005 N.

Motor de modelo: Se utiliza el motor con el fin de imprimir el movimiento de rotación al modelo, teniendo la posibilidad de seleccionar mediante una fuente regulable las velocidades de rotación deseadas. En el eje del mismo se conectan los cilindros para el ensayo mediante una rosca de conexión. 
Fuente: Fuente de corriente continua que permite regular la tensión de salida. De esta manera se pueden, seleccionar las velocidades de rotación del motor de CC según la necesidad requerida.

Tacómetro estroboscópico: Marca Lutron modelo DT-2239A. Se utiliza dicho instrumento para la medición de las velocidades de rotación de los modelos desde afuera del túnel a través de la ventana de acrílico.

Rango: 100 - 10000 rpm. Resolución: 1 rpm.

Barómetro: Marca Druck modelo DPI740. Se utiliza para conocer la presión atmosférica en la sección de pruebas.

Psicrómetro: Se utiliza para la determinación de las temperaturas de bulbo húmedo y seco. Mediante la medición de estos parámetros y la presión atmosférica se puede determinar la densidad del aire en la sección de pruebas.

\subsubsection{Caracterización}

En este apartado se presenta el estudio de caracterización del túnel de viento con el fin de conocer los perfiles de velocidad y la intensidad de la turbulencia en la sección de prueba a diferentes velocidades de la corriente.

\subsubsection{Velocidades medias}

Con un sistema de tubo Pitot, se midió la velocidad en nueve puntos de la sección de pruebas (figura 2.9) y se calculó la velocidad media a diferentes velocidades de la corriente.

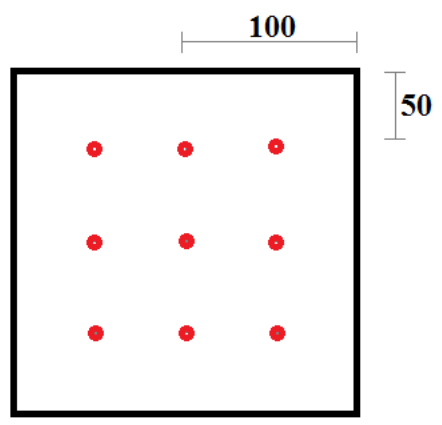

Figura 3.9 Puntos de medición en sección del túnel (unidades en mm). 
En la tabla 2.2 se presentan los resultados de las mediciones de los ensayos de caracterización, el cálculo de la velocidad media y la desviación estándar de las mediciones.

Tabla 2.2 Resultados de ensayos de medición de velocidades y calculos de velocidad media y desviación estandar.

\begin{tabular}{|c|c|c|c|c|}
\hline \multicolumn{3}{|c|}{$\begin{array}{c}\text { Velocidades } \\
\text { medidas } \\
{[\mathrm{m} / \mathrm{s}]}\end{array}$} & $\begin{array}{c}\text { Velocidad } \\
\text { media } \\
{[\mathrm{m} / \mathrm{s}]}\end{array}$ & $\begin{array}{c}\text { Desviación } \\
\text { estándar } \\
{[\mathrm{m} / \mathrm{s}]}\end{array}$ \\
\hline 1,99 & 2,18 & 1,99 & \multirow{3}{*}{2,20} & \multirow{3}{*}{0,29} \\
\hline 1,99 & 2,67 & 1,99 & & \\
\hline 2,36 & 2,67 & 1,99 & & \\
\hline 4,36 & 4,88 & 4,63 & \multirow{3}{*}{4,73} & \multirow{3}{*}{0,24} \\
\hline 4,54 & 5,19 & 4,71 & & \\
\hline 4,79 & 4,88 & 4,63 & & \\
\hline 6,48 & 6,84 & 6,17 & \multirow{3}{*}{6,47} & \multirow{3}{*}{0,41} \\
\hline 6,66 & 7,01 & 6,29 & & \\
\hline 6,66 & 6,48 & 5,63 & & \\
\hline 7,96 & 8,44 & 8,11 & \multirow{3}{*}{8,15} & \multirow{3}{*}{0,22} \\
\hline 8,06 & 8,54 & 8,16 & & \\
\hline 8,21 & 7,96 & 7,91 & & \\
\hline 8,99 & 9,75 & 9,21 & \multirow{3}{*}{9,35} & \multirow{3}{*}{0,26} \\
\hline 9,34 & 9,75 & 9,21 & & \\
\hline 9,42 & 9,34 & 9,12 & & \\
\hline
\end{tabular}

\subsubsection{Turbulencia}

Para el estudio de la turbulencia se utilizó un sistema de medición de hilo caliente DANTEC Streamline 90N10 con el cual se midió la velocidad de la corriente en el eje longitudinal, eje $x$ y en el perpendicular a este, eje $y$ (figura 2.10), de esta manera se determinó la intensidad de la turbulencia según el eje $x$.

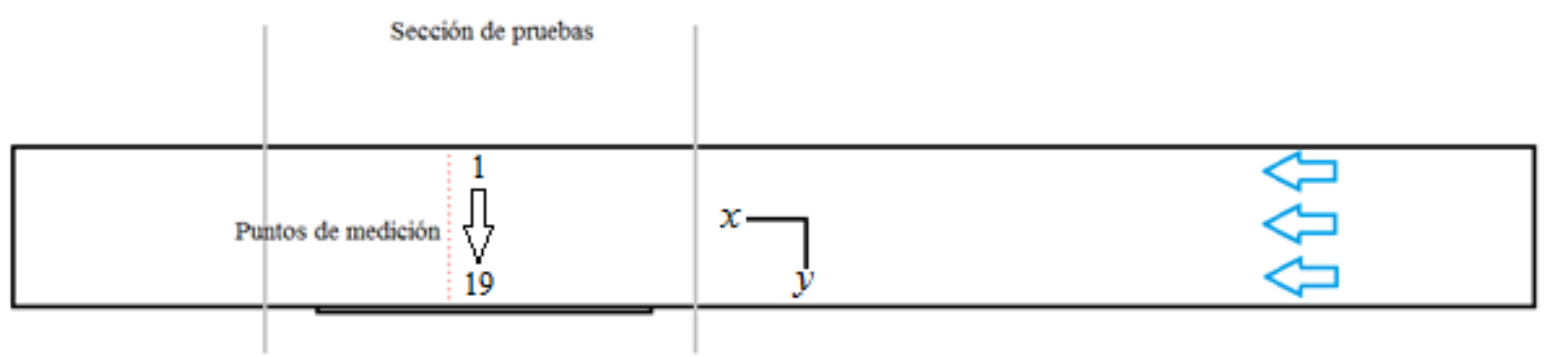

Figura 3.10 Sector donde se realizó la caracterización. 
Se tomaron mediciones en 19 puntos, según el eje $y$ del túnel a una altura correspondiente al centro de la sección, discretizando el ancho de la sección de pruebas del túnel cada $1 \mathrm{~cm}$, para cada velocidad de ensayo (ver apartado 2.5.1.). Para tal fin se dotó al túnel de una guía lineal externa con un soporte, que permitía movilizar el sensor de hilo caliente sin generar perturbaciones en las mediciones.

Los resultados se presentan en las tablas 2.3, 2.4, 2.5 y 2.6.

Tabla 2.3 Resultados ensayos de caracterización túnel $\left(\mathrm{V}_{\text {ref }}=5 \mathrm{~m} / \mathrm{s}\right)$.

\begin{tabular}{|c|c|c|c|}
\hline \multicolumn{4}{|c|}{ Velocidad referencia: $\mathbf{5 ~ m / s}$} \\
\hline \multirow{2}{*}{ Puntos } & $\boldsymbol{V}_{\text {ref } \boldsymbol{x} \text { media }}$ & $\boldsymbol{V}_{\text {ref } \boldsymbol{y} \text { media }}$ & Intensidad turbulencia (eje $\boldsymbol{x}$ ) \\
\cline { 2 - 4 } & {$[\mathbf{m} / \mathbf{s}]$} & {$[\mathbf{m} / \mathbf{s}]$} & {$[\%]$} \\
\hline 1 & 3,96 & $-0,04$ & 8,62 \\
\hline 2 & 4,44 & 0,01 & 6,27 \\
\hline 3 & 4,75 & 0,04 & 4,82 \\
\hline 4 & 4,91 & 0,07 & 3,62 \\
\hline 5 & 4,96 & 0,08 & 2,97 \\
\hline 6 & 4,95 & 0,07 & 2,81 \\
\hline 7 & 4,96 & 0,05 & 2,54 \\
\hline 8 & 5,00 & 0,04 & 2,56 \\
\hline 9 & 5,06 & 0,03 & 2,57 \\
\hline 10 & 5,12 & 0,02 & 2,56 \\
\hline 11 & 5,17 & 0,01 & 2,57 \\
\hline 12 & 5,19 & 0,00 & 2,58 \\
\hline 13 & 5,18 & 0,00 & 2,54 \\
\hline 14 & 5,09 & 0,00 & 2,74 \\
\hline 15 & 5,05 & 0,00 & 2,86 \\
\hline 16 & 4,90 & 0,00 & 3,90 \\
\hline 17 & 4,62 & 0,01 & 5,65 \\
\hline 18 & 4,16 & 0,02 & 7,32 \\
\hline 19 & 3,56 & 0,04 & 9,21 \\
\hline
\end{tabular}


Tabla 2.4 Resultados ensayos de caracterización túnel $\left(\mathrm{V}_{\text {ref }}=8 \mathrm{~m} / \mathrm{s}\right)$.

\begin{tabular}{|c|c|c|c|}
\hline \multicolumn{4}{|c|}{ Velocidad referencia: $\mathbf{8} \mathbf{~ m} / \mathbf{s}$} \\
\hline \multirow{2}{*}{ Puntos } & $\boldsymbol{V}_{\text {ref } \boldsymbol{x} \text { media }}$ & $\boldsymbol{V}_{\text {ref } \boldsymbol{y}}$ media & Intensidad turbulencia (eje $\boldsymbol{x}$ ) \\
\cline { 2 - 4 } & {$[\mathbf{m} / \mathbf{s}]$} & {$[\mathbf{m} / \mathbf{s}]$} & {$[\%]$} \\
\hline 1 & 6,21 & $-0,04$ & 8,28 \\
\hline 2 & 6,99 & 0,02 & 6,04 \\
\hline 3 & 7,43 & 0,08 & 4,66 \\
\hline 4 & 7,65 & 0,12 & 3,54 \\
\hline 5 & 7,69 & 0,13 & 3,10 \\
\hline 6 & 7,65 & 0,11 & 2,99 \\
\hline 7 & 7,62 & 0,08 & 2,61 \\
\hline 8 & 7,67 & 0,06 & 2,59 \\
\hline 9 & 7,77 & 0,04 & 2,72 \\
\hline 10 & 7,86 & 0,03 & 2,74 \\
\hline 11 & 7,95 & 0,00 & 2,71 \\
\hline 12 & 7,99 & $-0,01$ & 2,67 \\
\hline 13 & 7,96 & $-0,01$ & 2,65 \\
\hline 14 & 7,86 & $-0,01$ & 2,71 \\
\hline 15 & 7,73 & $-0,01$ & 2,89 \\
\hline 16 & 7,57 & $-0,01$ & 3,72 \\
\hline 17 & 7,16 & 0,00 & 5,36 \\
\hline 18 & 6,52 & 0,03 & 6,90 \\
\hline 19 & 5,54 & 0,05 & 8,81 \\
\hline
\end{tabular}


Tabla 2.5 Resultados ensayos de caracterización túnel $\left(\mathrm{V}_{\mathrm{ref}}=10 \mathrm{~m} / \mathrm{s}\right)$.

\begin{tabular}{|c|c|c|c|}
\hline \multicolumn{4}{|c|}{ Velocidad referencia: $\mathbf{1 0} \mathbf{~ m} / \mathbf{s}$} \\
\hline \multirow{2}{*}{ Puntos } & $\boldsymbol{V}_{\text {ref } \boldsymbol{x} \text { media }}$ & $\boldsymbol{V}_{\text {ref } \boldsymbol{y}}$ media & Intensidad turbulencia (eje $\boldsymbol{x}$ ) \\
\cline { 2 - 4 } & {$[\mathbf{m} / \mathbf{s}]$} & {$[\mathbf{m} / \mathbf{s}]$} & {$[\%]$} \\
\hline 1 & 8,37 & $-0,05$ & 8,28 \\
\hline 2 & 9,51 & 0,04 & 6,40 \\
\hline 3 & 10,12 & 0,11 & 4,82 \\
\hline 4 & 10,40 & 0,18 & 3,84 \\
\hline 5 & 10,46 & 0,19 & 3,38 \\
\hline 6 & 10,40 & 0,16 & 3,18 \\
\hline 7 & 10,34 & 0,11 & 2,89 \\
\hline 8 & 10,41 & 0,07 & 2,75 \\
\hline 9 & 10,53 & 0,05 & 2,92 \\
\hline 10 & 10,66 & 0,03 & 2,93 \\
\hline 11 & 10,78 & 0,00 & 2,96 \\
\hline 12 & 10,84 & $-0,02$ & 2,85 \\
\hline 13 & 10,81 & $-0,02$ & 2,87 \\
\hline 14 & 10,71 & $-0,01$ & 3,00 \\
\hline 15 & 10,58 & $-0,02$ & 3,02 \\
\hline 16 & 10,36 & $-0,03$ & 3,78 \\
\hline 17 & 9,87 & 0,00 & 5,44 \\
\hline 18 & 8,91 & 0,03 & 7,22 \\
\hline 19 & 7,59 & 0,07 & 9,06 \\
\hline
\end{tabular}


Tabla 2.6 Resultados ensayos de caracterización túnel $\left(\mathrm{V}_{\text {ref }}=12 \mathrm{~m} / \mathrm{s}\right)$.

\begin{tabular}{|c|c|c|c|}
\hline \multicolumn{4}{|c|}{ Velocidad referencia: $\mathbf{1 2} \mathbf{~ m} / \mathbf{s}$} \\
\hline \multirow{2}{*}{ Puntos } & $\boldsymbol{V}_{\text {ref } \boldsymbol{x}}$ media & $\boldsymbol{V}_{\text {ref } \boldsymbol{y}}$ media & Intensidad turbulencia (eje $\boldsymbol{x}$ ) \\
\cline { 2 - 4 } & {$[\mathbf{m} / \mathbf{s}]$} & {$[\mathbf{m} / \mathbf{s}]$} & {$[\mathbf{\%}]$} \\
\hline 1 & 9,52 & $-0,03$ & 8,37 \\
\hline 2 & 10,76 & 0,06 & 6,35 \\
\hline 3 & 11,50 & 0,16 & 4,84 \\
\hline 4 & 11,79 & 0,23 & 3,79 \\
\hline 5 & 11,83 & 0,24 & 3,54 \\
\hline 6 & 11,75 & 0,21 & 3,33 \\
\hline 7 & 11,71 & 0,14 & 3,06 \\
\hline 8 & 11,78 & 0,10 & 2,88 \\
\hline 9 & 11,93 & 0,06 & 3,08 \\
\hline 10 & 12,08 & 0,04 & 3,18 \\
\hline 11 & 12,21 & 0,01 & 3,10 \\
\hline 12 & 12,26 & $-0,02$ & 3,00 \\
\hline 13 & 12,27 & $-0,01$ & 3,01 \\
\hline 14 & 12,12 & 0,00 & 3,08 \\
\hline 15 & 11,97 & $-0,02$ & 3,09 \\
\hline 16 & 11,75 & $-0,02$ & 3,89 \\
\hline 17 & 11,16 & 0,00 & 5,52 \\
\hline 18 & 10,09 & 0,04 & 7,30 \\
\hline 19 & 8,53 & 0,08 & 9,13 \\
\hline
\end{tabular}

La intensidad de la turbulencia según el eje $x$ del túnel de viento se sitúa entre el 2 y $3 \%$ en la zona central donde se ubican los modelos. 


\subsection{MODELOS DE ENSAYO}

\subsubsection{Descripción}

Los modelos para los ensayos fueron fabricados a partir del mecanizado de teflón y acero, en función del modelo. El mecanizado constó de torneado y fresado mediante la utilización de un plato rotativo para poder mecanizar planos a diferentes ángulos.

Todos los modelos tienen mecanizado un orificio roscado en un plano con el fin de poder acoplar dicho modelo al eje del motor.

\subsubsection{Modelos ensayados}

Se mecanizaron catorce modelos y los mismos se detallan a continuación.

Las dimensiones se expresan en mm.

\section{Modelo 1 - Circular liso (CL)}

El mismo se encuentra mecanizado en teflón.
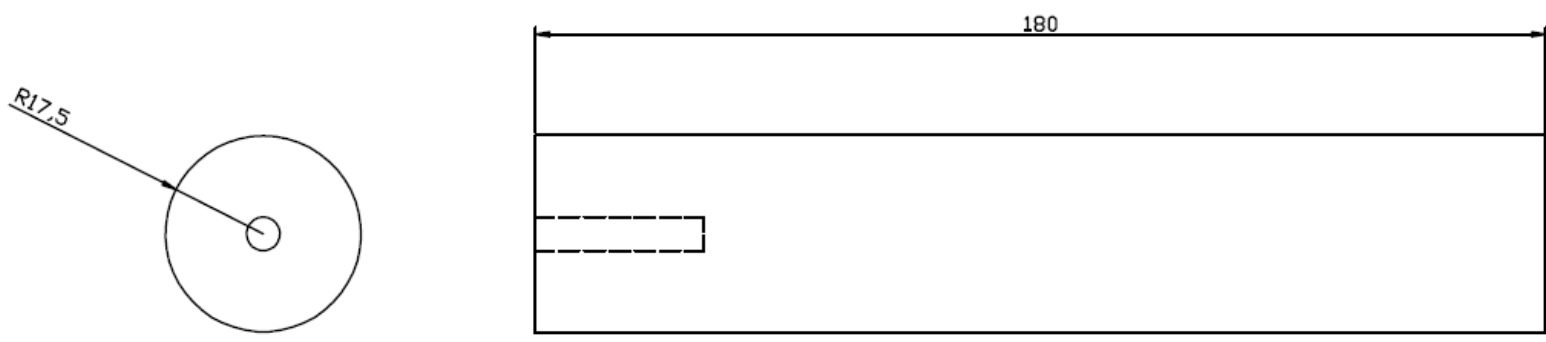

Figura 3.11 Modelo de cilindro.

\section{Modelo 2 - Circular recubierto con lija fina (CLF)}

El mismo tiene la misma geometría que el Modelo 1, se encuentra mecanizado en teflón y está recubierto con tela esmeril de grano fino.

\section{Modelo 3 - Circular recubierto con lija gruesa (CLG)}

El mismo tiene la misma geometría que el Modelo 1, se encuentra mecanizado en teflón y está recubierto con tela esmeril de grano grueso. 


\section{Modelo 4 - Circular calado (CC)}

El mismo tiene la misma geometría que el Modelo 1, se encuentra mecanizado en teflón y tiene caladuras fresadas cada $90^{\circ}$ de $3 \mathrm{~mm}$ profundidad y $3 \mathrm{~mm}$ de ancho.
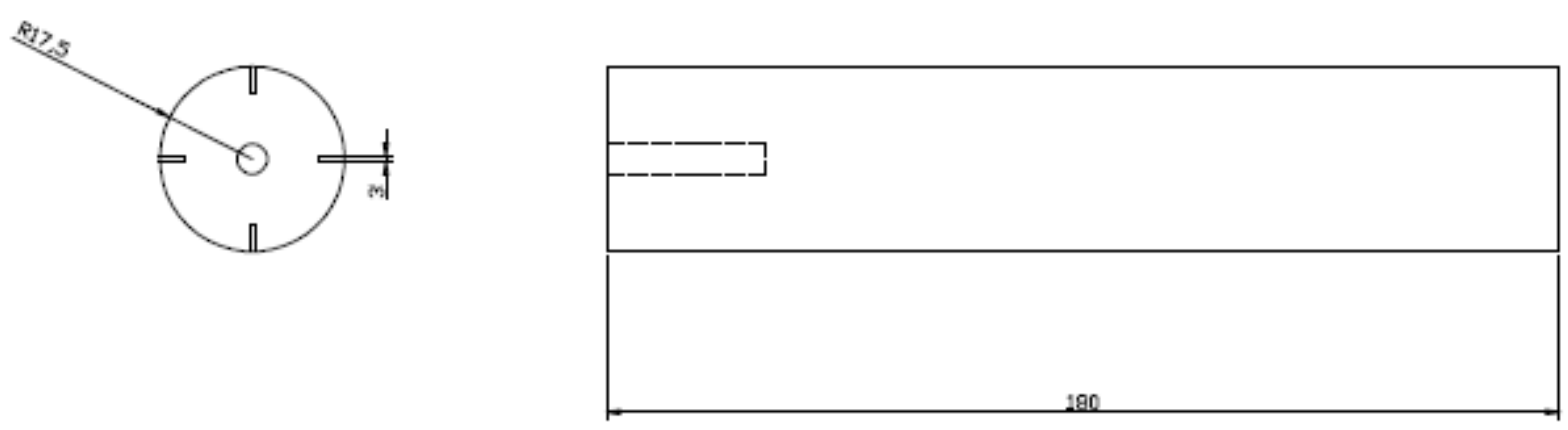

Figura 3.12 Modelo de cilindro calado.

\section{Modelo 5 - Circular con aletas (CA)}

Es el mismo modelo que el número 4 sólo que en sus caladuras se insertan listones de madera balsa de $3 \mathrm{~mm}$ de espesor y $6 \mathrm{~mm}$ de ancho con el fin de generar unas aletas de $3 \mathrm{~mm}$ de altura desde la superficie del cilindro.
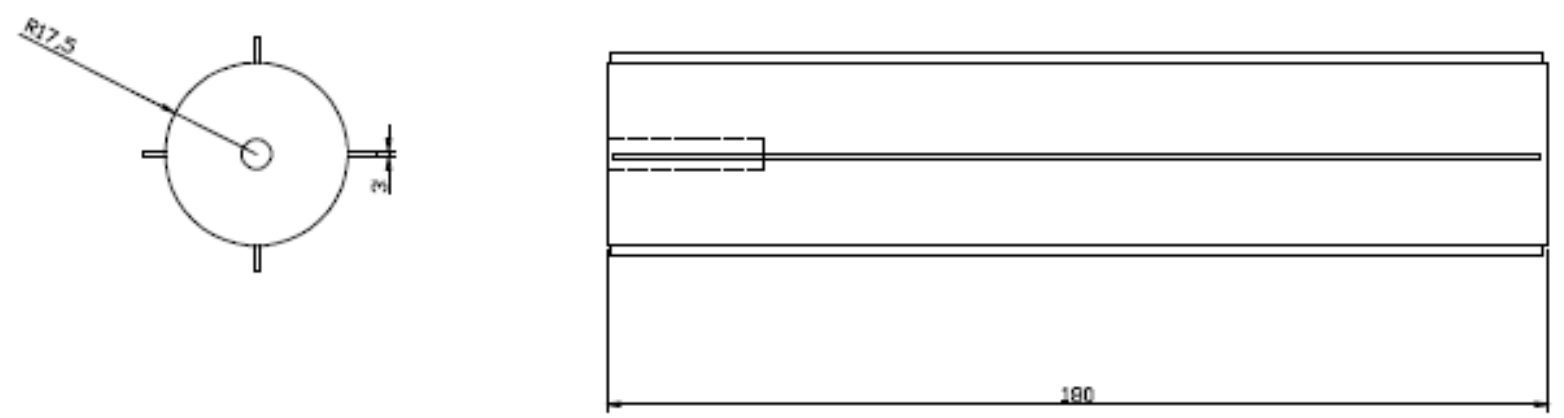

Figura 3.13 Modelo de cilindro con aletas. 


\section{Modelo 6 - Decágono (D)}

El mismo se encuentra mecanizado en teflón.
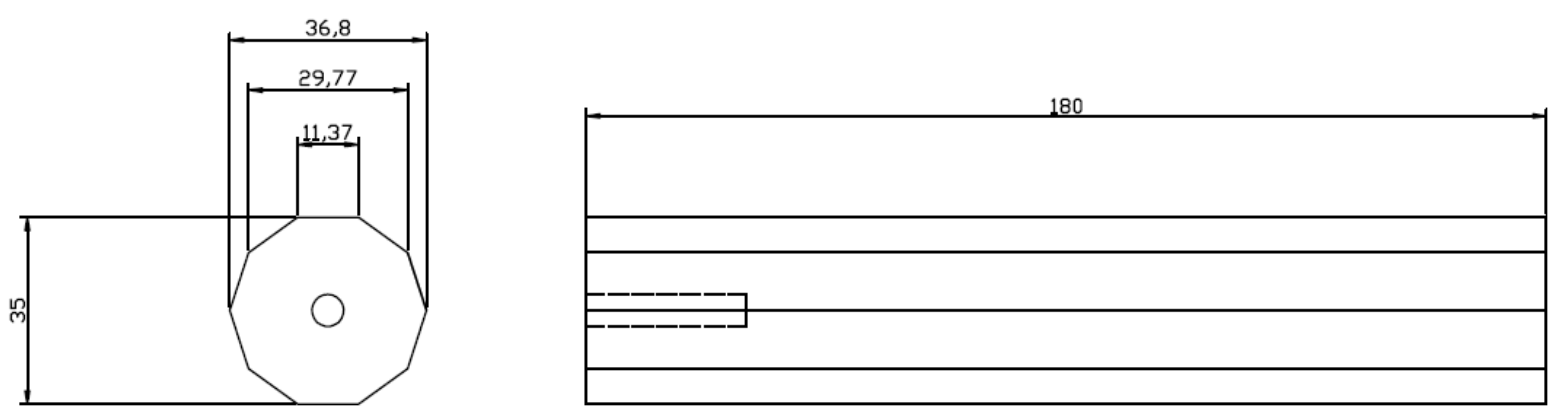

Figura 3.14 Modelo de Decágono.

\section{Modelo 7 - Octógono (O)}

El mismo se encuentra mecanizado en teflón.
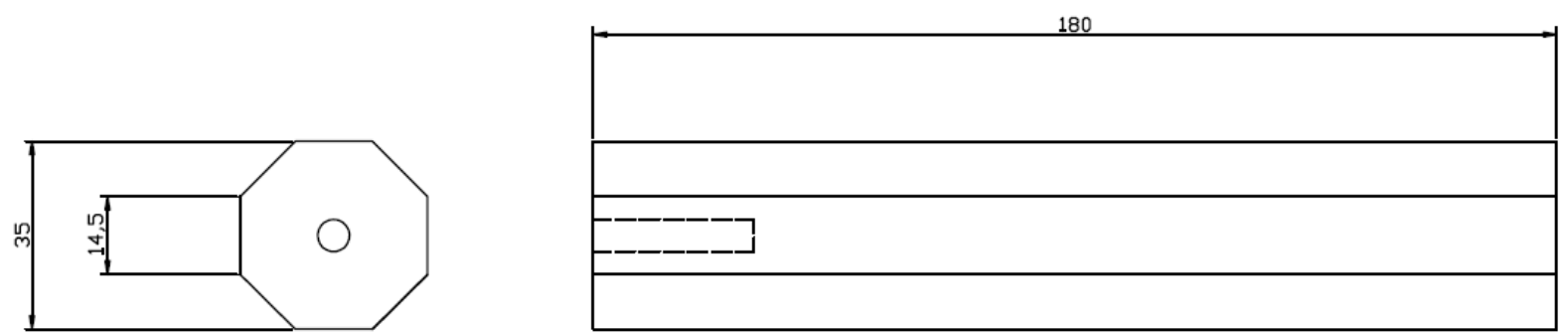

Figura 3.15 Modelo de octógono.

\section{Modelo 8 - Hexágono (H)}

El mismo se encuentra mecanizado en teflón.
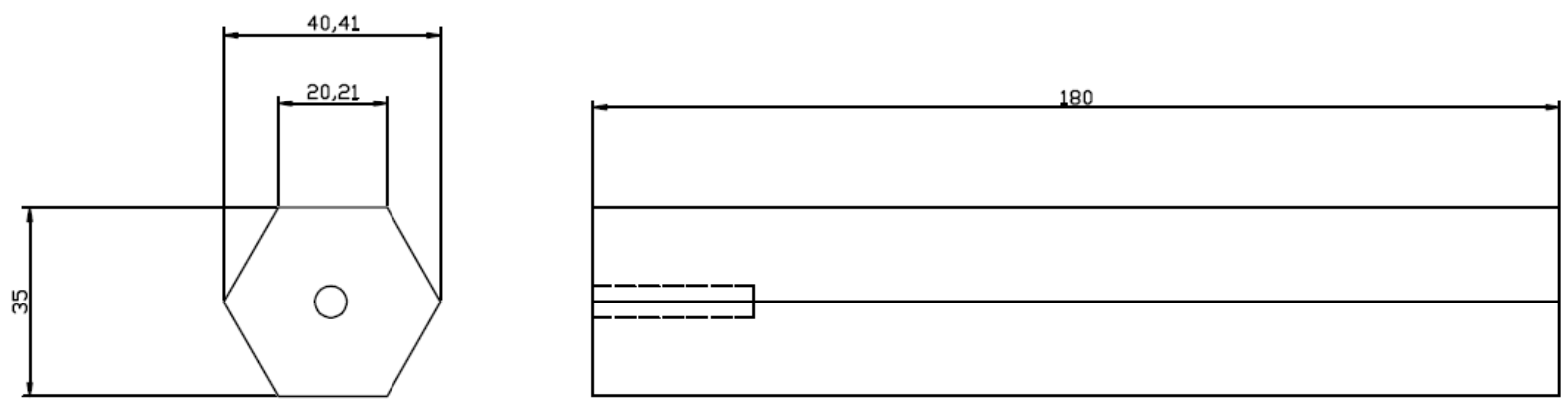

Figura 3.16 Modelo de hexágono. 


\section{Modelo 9 - Cuadrado (CD)}

El mismo se encuentra mecanizado en téflon.
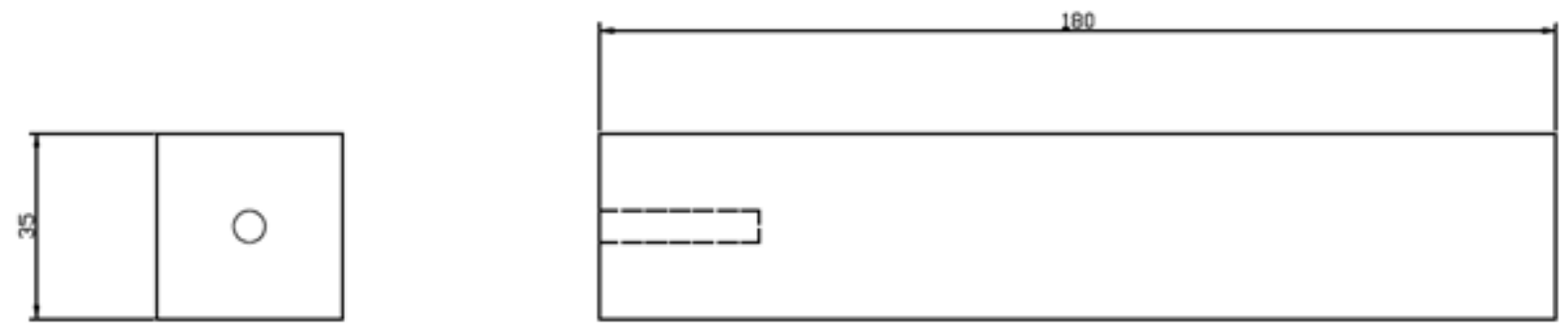

Figura 3.17 Modelo de cuadrado.

\section{Modelo 10 - Cuadrado con aristas redondeadas (CAR)}

El mismo se encuentra mecanizado en teflón. Tiene la particularidad de poseer las aristas redondeadas para lo que se construyó una herramienta especial para lograr dicho mecanizado.
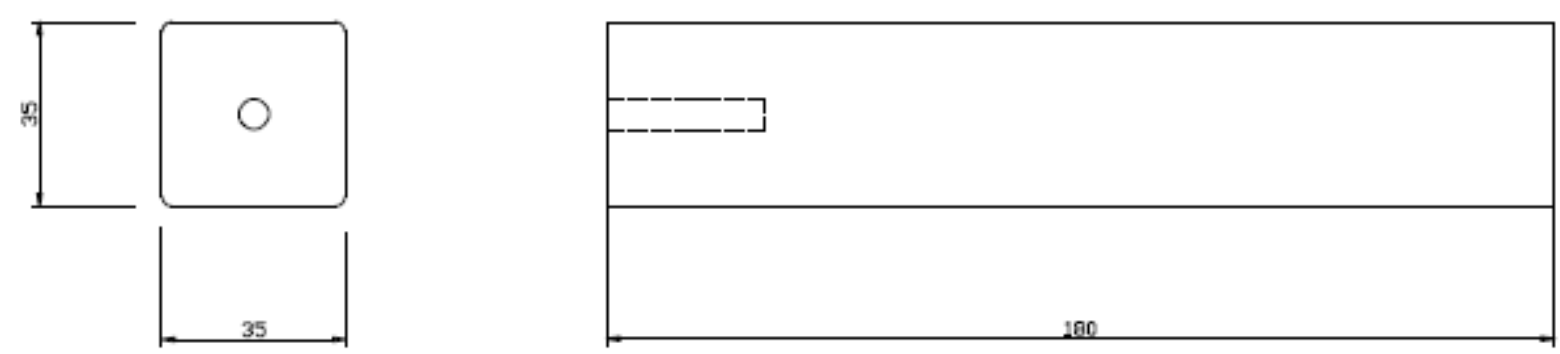

Figura 3.18 Modelo de cuadrado con aristas redondeadas.

\section{Modelo 11 - Triángulo (T)}

El mismo se encuentra mecanizado en teflón.
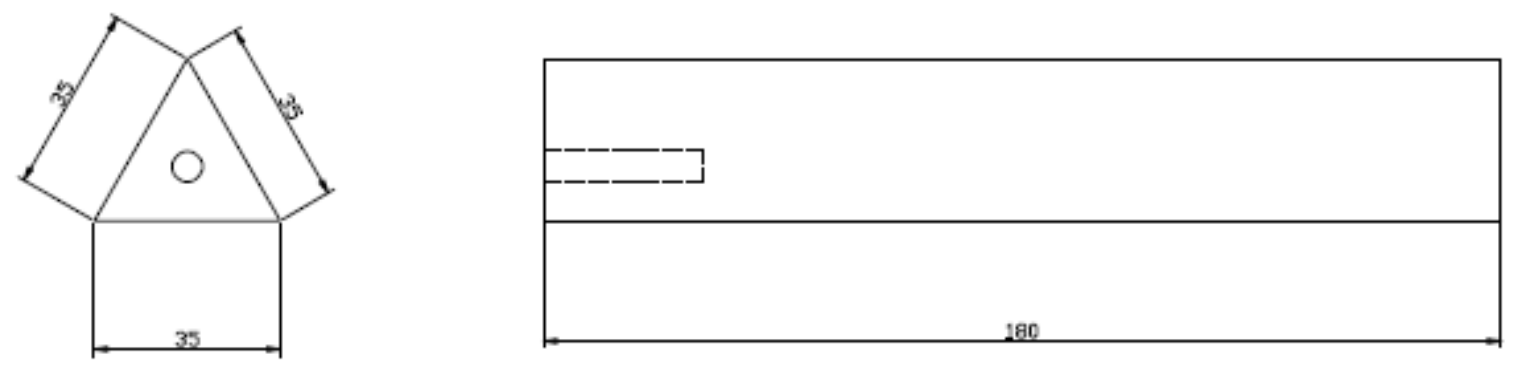

Figura 3.19 Modelo de triángulo. 


\section{Modelo 12 - Triángulo con aristas redondeas (TAR)}

Mecanizado en teflón. Tiene la particularidad de poseer las aristas redondeadas para lo que se construyó una herramienta especial para lograr dicho mecanizado.
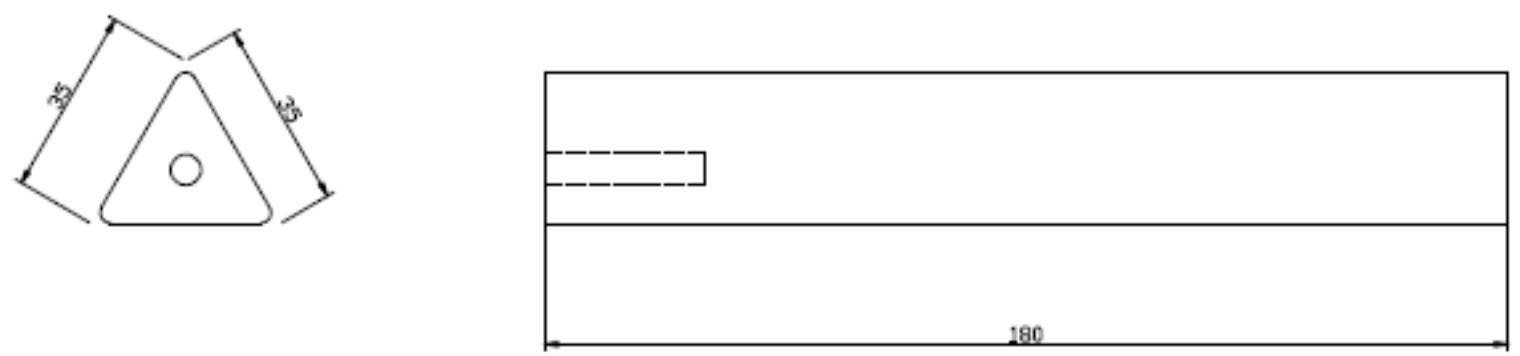

Figura 3.20 Modelo de triángulo con aristas redondeadas.

\section{Modelo 13 - Placa plana gruesa (PPG)}

Para mayor facilidad y precisión, el mismo fue mecanizado en acero.
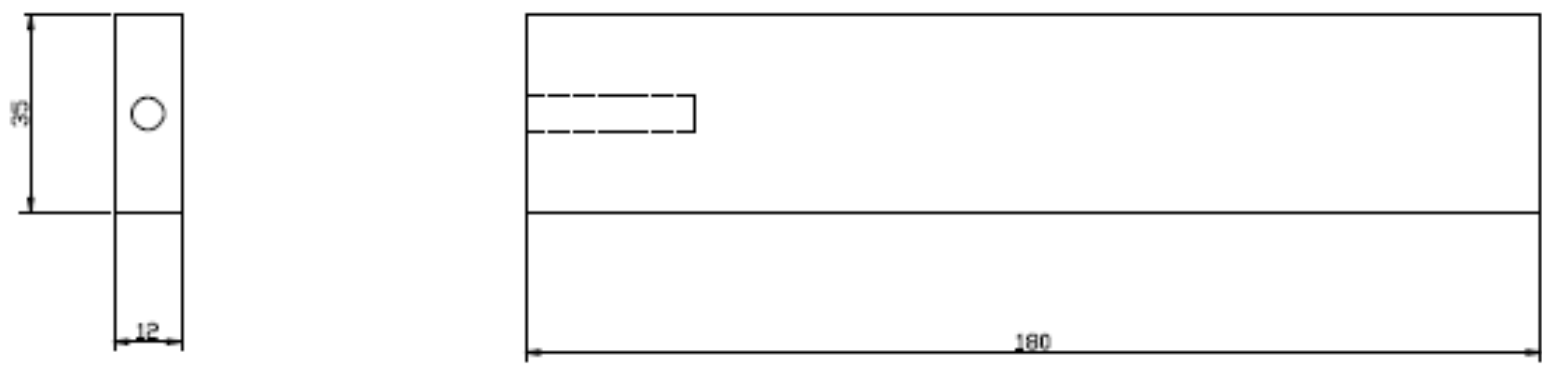

Figura 3.21 Modelo de placa plana gruesa.

\section{Modelo 14 - Placa plana fina (PPF)}

Mecanizada en acero. Tiene $3 \mathrm{~mm}$ de espesor y en su parte inferior la sección se ensancha para poder ser sujetada como los otros modelos.
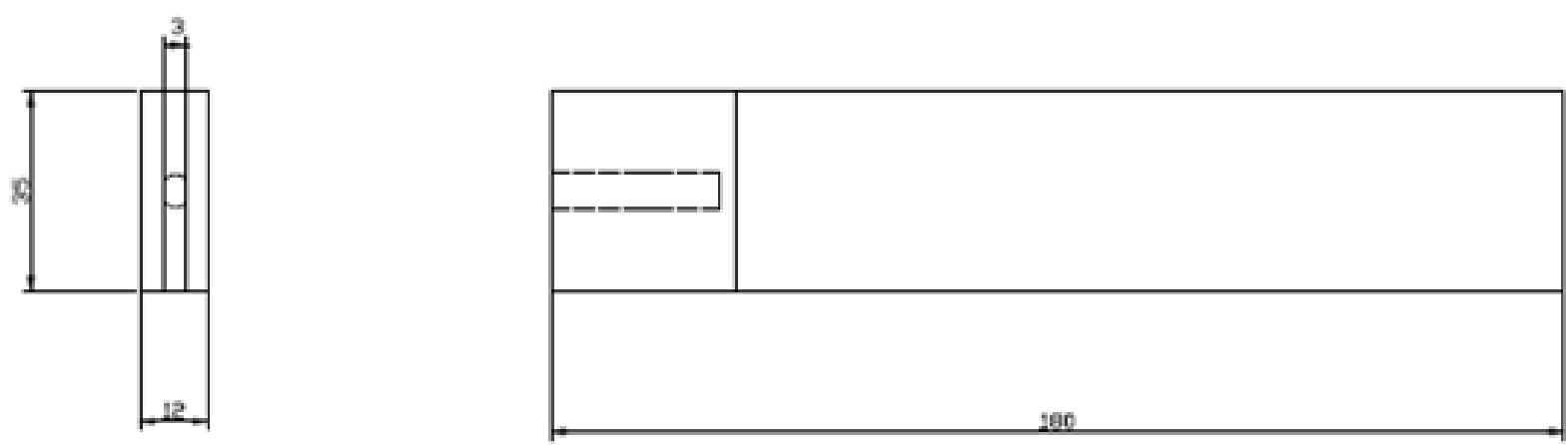

Figura 3.22 Modelo de placa plana fina. 


\subsection{ENSAYOS}

Se realizaron dos tipos de ensayo. Uno de mediciones de fuerzas de sustentación y resistencia en función de la velocidad de giro y de la velocidad de la corriente libre, con el objeto de caracterizar aerodinámicamente los cuerpos; y otro de visualizaciones, con el fin de conocer los diversos patrones que adopta el flujo en el entorno de los cuerpos.

En el capítulo 3 los resultados de ambos ensayos son utilizados para tratar de comprender el flujo entorno a los cuerpos y determinar el comportamiento aerodinámico de cada cuerpo en rotación inmerso en una corriente de aire.

\subsubsection{Ensayos de mediciones de fuerzas}

\subsubsection{Procedimientos de ensayo}

A continuación se detalla el procedimiento de ensayo para la medición de sustentación y resistencia:

I. Se realiza la conexión del modelo al eje del motor. Para tal fin, se accede desde la tapa superior y se atornilla al mismo al eje roscado para luego sujetar mediante una contratuerca.

II. Se verifica que no haya desalineaciones y desbalanceo para todas las velocidades de giro de los ensayos (tabla 2.7).

III. Se fija la velocidad de giro deseada mediante el regulador de tensión y la lámpara estroboscópica, y se tara la balanza.

IV. Se enciende el variador de frecuencia y se ajusta la velocidad del soplador a una velocidad de la corriente libre deseada.

V. Una vez estabilizado el flujo (verificación mediante el micromanómetro) se toma nota de la indicación de la balanza, ya sea para sustentación o resistencia.

VI. Luego se ajusta el variador a una velocidad de la corriente libre superior, manteniendo la velocidad de giro del modelo y se procede de la misma manera hasta lograr el barrido deseado (tabla 2.8).

VII. Luego se vuelve al punto III y se aumenta la velocidad de giro del modelo y se repite el ensayo. 
Las velocidades de giro ensayadas se presentan en la tabla 2.7.

Tabla 2.7 Velocidades de giro para cada ensayo.

\begin{tabular}{|c|c|}
\hline $\begin{array}{c}\omega \\
\text { Ensayo de sustentación } \\
{[\mathbf{r p m}]}\end{array}$ & $\begin{array}{c}\boldsymbol{\omega} \\
\text { Ensayo de resistencia } \\
{[\mathbf{r p m}]}\end{array}$ \\
\hline- & 0 \\
\hline 1950 & 1950 \\
\hline 2310 & 2310 \\
\hline 2520 & 2520 \\
\hline 2725 & 2725 \\
\hline
\end{tabular}

Se realizó un barrido de velocidad de la corriente libre para las velocidades que se muestran en la tabla 2.8 .

Tabla 2.8 Velocidad de la corriente libre para cada ensayo.

\begin{tabular}{|c|}
\hline $\begin{array}{c}\boldsymbol{v} \\
{[\mathbf{m} / \mathbf{s}]}\end{array}$ \\
\hline 5 \\
\hline 8 \\
\hline 10 \\
\hline 12 \\
\hline
\end{tabular}

\subsubsection{Consideraciones}

Previo a mencionar las consideraciones utilizadas para la obtención de resultados se define:

Coeficiente de sustentación:

$C_{L}=\frac{2 L}{\rho v^{2} S}$

Coeficiente de resistencia:

$C_{D}=\frac{2 D}{\rho v^{2} S}$ 
Velocidad tangencial:

$u=\omega R$

Número de Reynolds:

$$
\mathrm{R}_{\mathrm{e}}=\frac{v \phi}{v}
$$

donde

- $\quad L$ : Sustentación

- $\quad D$ : Resistencia

- $\rho$ : Densidad del aire

- $\quad v$ : Velocidad del aire

- $\quad S$ : Superficie del cuerpo

- $\omega$ : velocidad de giro

- $R$ : Radio del cuerpo

- $\phi$ : Diámetro del cuerpo

- $\quad v$ : Viscosidad cinemática del aire

Un punto de importancia relevante es definir el diámetro de referencia y la superficie frontal para los cálculos de $C_{L}$ y $C_{D}$ por su influencia en los resultados. Después de estudiar el problema se decidió tomar como criterio el diámetro del círculo medio entre el círculo inscripto y el círculo circunscrito de los cuerpos. Este criterio se tuvo en cuenta en todos los cuerpos salvo en los circulares y en las placas, que se tomó como diámetro de referencia el del círculo circunscrito (figura 2.23).

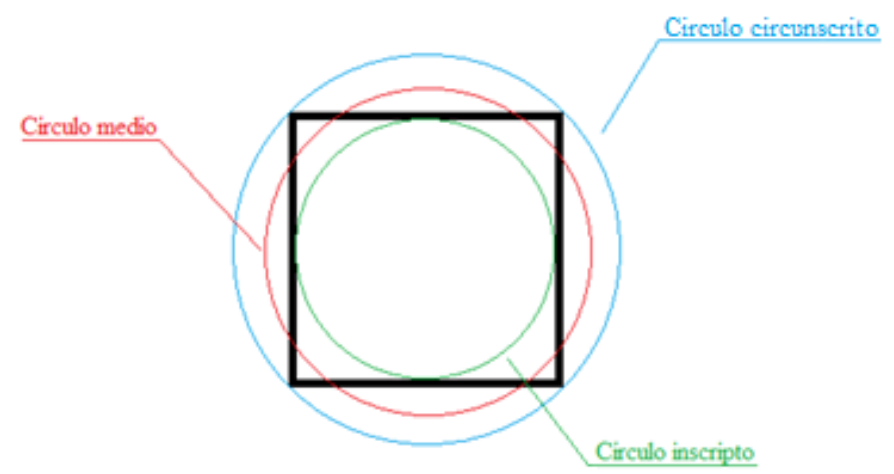

Figura 3.23 Círculos de referencia. 
En la tabla 2.9 se presentan los diámetros de referencia utilizados para el cálculo de los coeficientes aerodinámicos y el rango de número de Reynolds en los que se hicieron los ensayos para cada cuerpo.

Tabla 2.9 Diámetros de referencia y rango de número de Reynolds de los cuerpos.

\begin{tabular}{|c|c|c|c|c|}
\hline Acrónimo & Cuerpo & $\begin{array}{c}\boldsymbol{\phi} \\
{[\mathbf{m}]}\end{array}$ & $\begin{array}{c}\mathbf{R}_{\mathbf{e}} \\
\mathbf{m i ́ n .}\end{array}$ & $\begin{array}{c}\mathbf{R}_{\mathbf{e}} \\
\mathbf{m a ́ x} .\end{array}$ \\
\hline CL & Circular liso & 0,0350 & 13700 & 29700 \\
\hline CLF & Circular recubierto con lija fina & 0,0350 & 13700 & 29700 \\
\hline CLG & Circular recubierto con lija gruesa & 0,0350 & 13700 & 29700 \\
\hline CC & Circular calado & 0,0350 & 13700 & 29700 \\
\hline CA & Circular con aletas & 0,0380 & 14900 & 32000 \\
\hline D & Decágono & 0,0360 & 14000 & 30500 \\
\hline O & Octógono & 0,0365 & 14300 & 30900 \\
\hline H & Hexágono & 0,0375 & 14700 & 31800 \\
\hline CD & Cuadrado & 0,0420 & 16200 & 35100 \\
\hline CAR & Cuadrado con aristas redondeadas & 0,0415 & 16200 & 35100 \\
\hline T & Triángulo & 0,0325 & 12700 & 27500 \\
\hline TAR & Triángulo con aristas redondeadas & 0,0295 & 11500 & 25000 \\
\hline PPG & Placa plana gruesa & 0,0350 & 13700 & 29700 \\
\hline PPF & Placa plana fina & 0,0350 & 13700 & 29700 \\
\hline
\end{tabular}

\subsubsection{Resultados}

Un sencillo análisis dimensional muestra que uno de los parámetros adimensionales que debe influir en el problema es la relación entre la velocidad periférica del cuerpo, debido a la rotación del mismo $u=\omega R$, y la velocidad de la corriente libre $v$. Este parámetro se utiliza frecuentemente en la literatura, y se emplea en la presente tesis.

Por tal, a modo de ejemplo, a continuación se presentan los resultados de los ensayos para el decágono, y en el Anexo I se presentan todos los resultados de los ensayos, para cada cuerpo, de acuerdo al siguiente formato:

- Tabla con resultados de ensayos de sustentación, resistencia y cálculos de coeficientes $C_{L}, C_{D}$ y relación de velocidades, $u / v$

- Gráfico de sustentación, $L$, vs velocidad de la corriente libre, $v$

- Gráfico de coeficiente de sustentación, $C_{L}$, vs relación de velocidades, $u / v$ 
- Gráfico de resistencia, $D$, vs velocidad de la corriente libre, $v$

- Gráfico de coeficiente de resistencia, $C_{D}$, vs relación de velocidades, $u / v$.

Cabe señalar que los resultados que se presentan a continuación no están corregidos por efectos de bloqueo en el túnel de viento, ya que en esta instancia el análisis es comparativo entre los diferentes cuerpos. En el apartado 2.6 se presenta un estudio de los efectos de bloqueo para los ensayos que se realizaron para la presente tesis.

Se detalla en la tabla 2.10 los resultados correspondientes a los ensayos de sustentación y resistencia en el decágono.

Tabla 2.10 Ejemplo de resultados correspondiente a los ensayos de sustentación y resistencia.

\begin{tabular}{|c|c|c|c|c|c|c|c|c|}
\hline \multicolumn{9}{|c|}{ Decágono $(\phi=0,036 \mathrm{~m})$} \\
\hline $\begin{array}{c}\omega \\
{[\mathbf{r p m}]}\end{array}$ & $\begin{array}{c}v \\
{[\mathrm{~m} / \mathrm{s}]}\end{array}$ & $\begin{array}{c}L \\
{[\mathbf{N}]}\end{array}$ & $C_{L}$ & $u / v$ & $\begin{array}{c}v \\
{[\mathrm{~m} / \mathrm{s}]}\end{array}$ & $\begin{array}{c}D \\
{[\mathrm{~N}]}\end{array}$ & $C_{D}$ & $u / v$ \\
\hline \multirow[t]{4}{*}{0} & - & - & - & - & 5,27 & 0,11 & 1,02 & 0,00 \\
\hline & - & - & - & - & 7,82 & 0,24 & 1,01 & 0,00 \\
\hline & - & - & - & - & 10,30 & 0,42 & 1,00 & 0,00 \\
\hline & - & - & - & - & 11,43 & 0,52 & 1,02 & 0,00 \\
\hline \multirow[t]{4}{*}{1950} & 5,27 & 0,13 & 1,20 & 0,70 & 5,27 & 0,06 & 0,56 & 0,70 \\
\hline & 7,88 & 0,18 & 0,72 & 0,47 & 7,87 & 0,15 & 0,62 & 0,47 \\
\hline & 10,27 & 0,23 & 0,56 & 0,36 & 10,34 & 0,28 & 0,67 & 0,36 \\
\hline & 11,44 & 0,27 & 0,52 & 0,32 & 11,50 & 0,35 & 0,68 & 0,32 \\
\hline \multirow[t]{4}{*}{2310} & 5,27 & 0,17 & 1,53 & 0,83 & 5,27 & 0,06 & 0,56 & 0,83 \\
\hline & 7,88 & 0,22 & 0,91 & 0,55 & 7,87 & 0,14 & 0,58 & 0,55 \\
\hline & 10,27 & 0,26 & 0,63 & 0,42 & 10,30 & 0,26 & 0,63 & 0,42 \\
\hline & 11,40 & 0,29 & 0,57 & 0,38 & 11,46 & 0,34 & 0,66 & 0,38 \\
\hline \multirow[t]{4}{*}{2520} & 5,27 & 0,18 & 1,67 & 0,90 & 5,27 & 0,06 & 0,56 & 0,90 \\
\hline & 7,88 & 0,25 & 1,03 & 0,60 & 7,87 & 0,15 & 0,60 & 0,60 \\
\hline & 10,27 & 0,29 & 0,71 & 0,46 & 10,34 & 0,26 & 0,62 & 0,46 \\
\hline & 11,44 & 0,32 & 0,62 & 0,42 & 11,46 & 0,34 & 0,66 & 0,41 \\
\hline \multirow[t]{4}{*}{2725} & 5,27 & 0,20 & 1,85 & 0,97 & 5,27 & 0,06 & 0,56 & 0,98 \\
\hline & 7,88 & 0,28 & 1,16 & 0,65 & 7,87 & 0,15 & 0,60 & 0,65 \\
\hline & 10,27 & 0,33 & 0,79 & 0,50 & 10,30 & 0,26 & 0,63 & 0,50 \\
\hline & 11,44 & 0,35 & 0,69 & 0,45 & 11,43 & 0,34 & 0,66 & 0,45 \\
\hline
\end{tabular}

En las figuras 2.24, 2.25, 2.26 y 2.27 se representan gráficamente los resultados de la tabla 2.10 . 


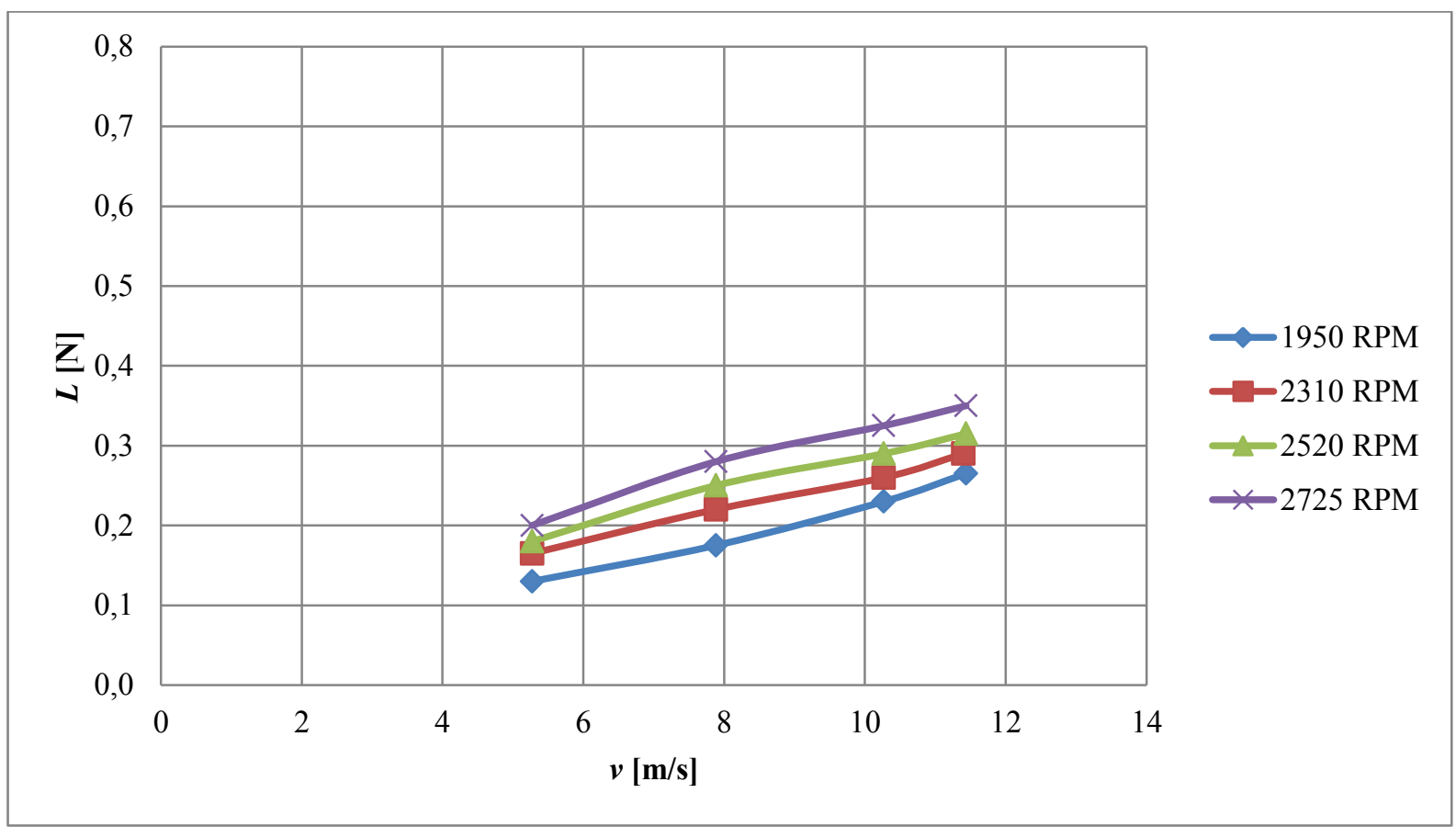

Figura 3.24 Variación de la sustentación $L$ en función de la velocidad de la corriente $v$ para cada velocidad de giro $\omega$. Decágono.

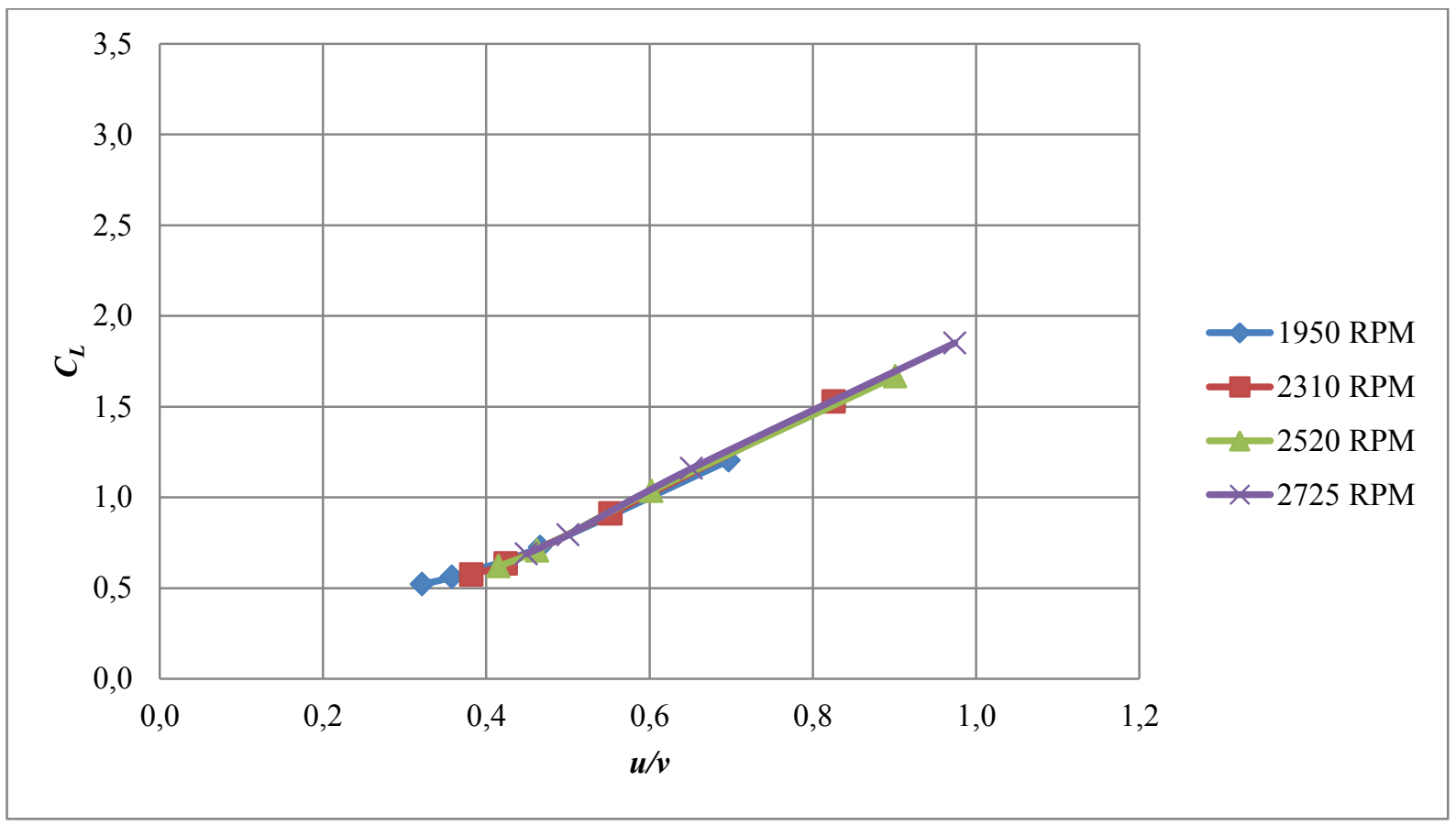

Figura 3.25 Variación del coeficiente de sustentación $C_{L}$ en función de la relación de velocidades $u / v$ para cada velocidad de giro $\omega$. Decágono. 


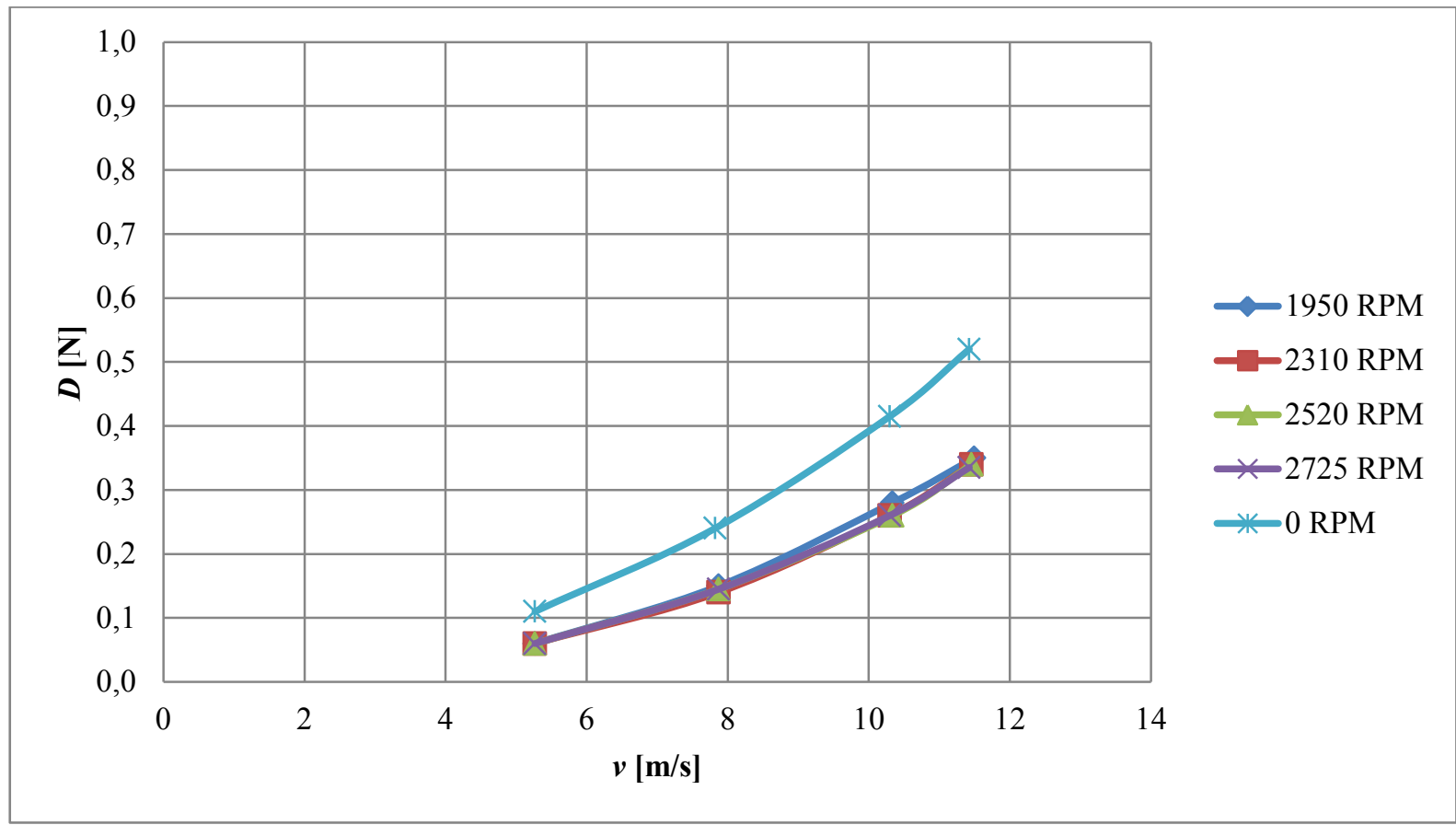

Figura 3.26 Variación de la resistencia $D$ en función de la velocidad de la corriente $v$ para cada velocidad de giro $\omega$. Decágono.

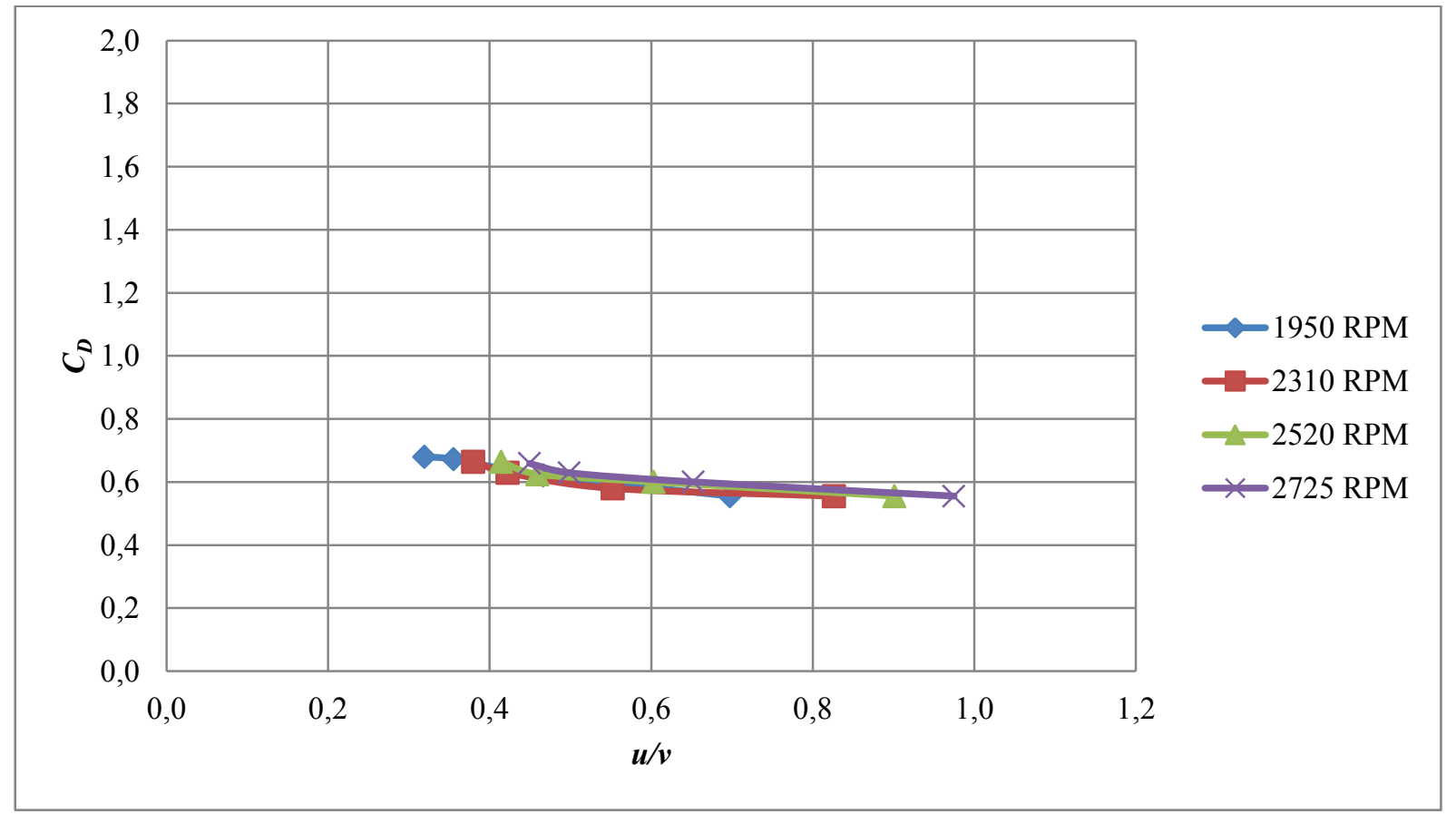

Figura 3.27 Variación del coeficiente de resistencia $C_{D}$ en función de la relación de velocidades $u / v$ para cada velocidad de giro $\omega$. Decágono. 
Las figuras 2.24 y 2.26 son el producto directo de las mediciones realizadas en el túnel de viento. Así mismo, las figuras 2.25 y 2.27 son producto de la adimensionalización de los valores medidos y son de vital importancia para conocer el comportamiento o patrón del flujo de los cuerpos al variar la relación de velocidades $u / v$.

Estos resultados fueron obtenidos para todos los cuerpos mencionados en el apartado $2.4 .2 \mathrm{y}$ los mismos se encuentran descriptos en el Anexo I.

En la tabla 2.11 se muestra un resumen de los resultados de los ensayos para cada cuerpo, en cuanto a su comportamiento.

Cuando en la tabla 2.11 se refiere a "forma de abanico leve" significa que las curvas de $C_{L}$ o $C_{D}$ respecto a la relación de velocidades $u / v$, poseen diferentes comportamientos para cada velocidad de giro $\omega$.

Tabla 2.11. Resumen correspondiente a los ensayos de sustentación y resistencia.

\begin{tabular}{|l|c|c|c|c|}
\hline \multirow{2}{*}{ Cuerpo } & \multicolumn{2}{|c|}{ Figura } & \multicolumn{2}{c|}{ Comentario de las curvas } \\
\cline { 2 - 5 } C & $\boldsymbol{C}_{\boldsymbol{L}}-\boldsymbol{u} / \boldsymbol{v}$ & $\boldsymbol{C}_{\boldsymbol{D}} \boldsymbol{-} \boldsymbol{u} \boldsymbol{v}$ & $\boldsymbol{C}_{\boldsymbol{L}}-\boldsymbol{u} / \boldsymbol{v}$ & $\boldsymbol{C}_{\boldsymbol{D}}-\boldsymbol{u} \boldsymbol{v}$ \\
\hline CLF & A1.2 & A1.4 & Un comportamiento & Un comportamiento \\
\hline CLG & $\mathrm{A} 1.10$ & $\mathrm{~A} 1.8$ & Un comportamiento & $\begin{array}{c}\text { Un comportamiento } \\
\text { irregular }\end{array}$ \\
\hline CC & A1.14 & A1.16 & Forma de abanico leve & Un comportamiento \\
\hline CA & A1.18 & A1.20 & Un comportamiento & $\begin{array}{c}\text { Dos comportamientos } \\
\text { (2725 rpmportamientos }- \text { el resto) }\end{array}$ \\
\hline D & A1.22 & A1.24 & Un comportamiento & Un comportamiento \\
\hline O & A1.26 & A1.28 & Un comportamiento & Un comportamiento \\
\hline H & A1.30 & A1.32 & Un comportamiento & Un comportamiento \\
\hline CD & A1.34 & A1.36 & Un comportamiento & Un comportamiento \\
\hline CAR & A1.38 & A1.40 & Un comportamiento & Un comportamiento \\
\hline T & A1.42 & A1.44 & Un comportamiento & $\begin{array}{c}\text { Un comportamiento } \\
\text { irregular }\end{array}$ \\
\hline TAR & A1.46 & A1.48 & Un comportamiento & Un comportamiento \\
\hline PPG & A1.50 & A1.52 & Un comportamiento & $\begin{array}{c}\text { Dos comportamientos } \\
\text { (1950 rpm - el resto) }\end{array}$ \\
\hline PPF & A1.54 & A1.56 & Forma de abanico leve & $\begin{array}{c}\text { Un comportamiento } \\
\text { irregular }\end{array}$ \\
\hline
\end{tabular}




\subsubsection{Ensayos de visualización de flujo alrededor de los cuerpos}

Con el fin de estudiar y analizar el comportamiento del flujo alrededor de los cuerpos ensayados se realizaron ensayos de visualización a partir de la utilización de humo, una luz estroboscópica y una cámara de alta velocidad (ver punto 2.5.2.1).

Se reformó el túnel de viento y se colocó una tapa superior de vidrio en la sección de pruebas con el objeto de facilitar el acceso visual a una cámara de fotos. Por la ventana lateral se instaló una luz estroboscópica y se procedió a opacar la sección de pruebas con el fin de mejorar el contraste en las fotografías. A su vez, se dotó el túnel con un sistema de generación de hilo de humo corriente arriba de la ubicación del modelo.

El sistema completo puede visualizarse en los esquemas de las figuras 2.28 y 2.29.

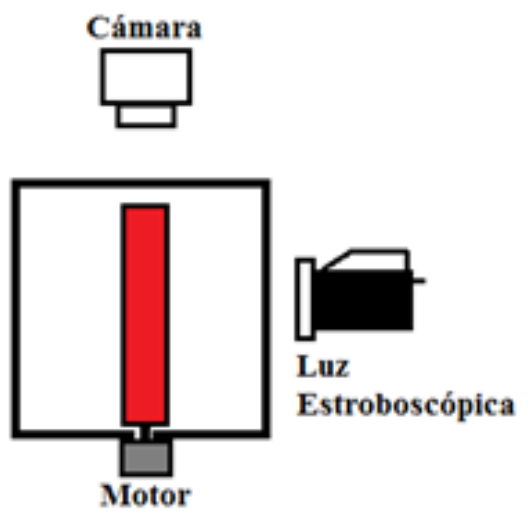

Figura 3.28 Sistema de obtención de fotos.

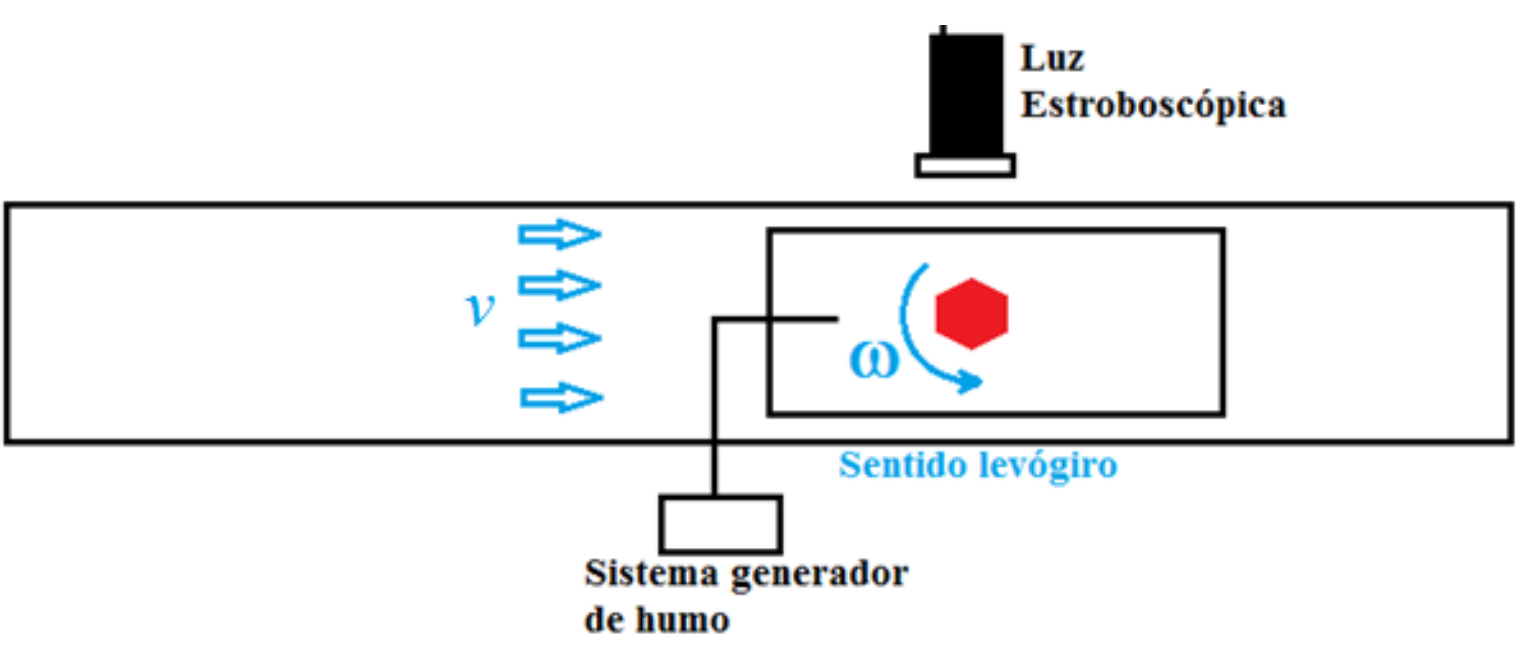

Figura 3.29 Sistema completo de visualización y obtención de fotos. 


\subsubsection{Equipamiento y características de las fotografías}

Para la realización de fotografías se utilizó el siguiente equipamiento.

- Cámara marca Nikon modelo D7000

- Máquina de humo Marca MLB modelo Z400

- Luz estroboscópica marca Lutrón modelo DT-2239A

Las fotografías se obtuvieron bajo las siguientes condiciones.

- Tiempo de exposición: $1 / 30 \mathrm{~s}$

- Velocidad ISO: ISO-3200

- Punto F: f/4.5

- Distancia focal: $40 \mathrm{~mm}$

- Apertura máxima: 4.4

\subsubsection{Procedimiento de ensayo}

Se realizaron ensayos sobre cada cuerpo a diferentes velocidades de la corriente libre y de rotación.

Con el objetivo de lograr visualizaciones precisas de los patrones de flujo se procedió a fijar la velocidad de la corriente libre y de rotación en un valor deseado. Luego se equiparó la frecuencia de la luz estroboscópica con la correspondiente a la velocidad de giro del cuerpo. De esta manera se logra una imagen del cuerpo "congelado" en una posición. Una vez realizado este paso se activa el sistema de humo y se procede a la toma de fotografías.

En todos los cuerpos se procedió a "congelar" la imagen para las diferentes posiciones de los mismos y se obtuvieron imágenes de los patrones de flujo para cada uno, para un instante de su giro determinado.

Antes de definir las condiciones de $\omega$ y $v$ para las fotografías se visualizó el flujo en todos las situaciones de ensayo que se presentaron en el apartado 2.5.1. Finalmente se eligieron condiciones de $\omega$ y $v$ para cada cuerpo en las cuales se observan patrones de flujo definidos. 


\subsubsection{Visualizaciones}

A modo de ejemplo, se presentan a continuación, imágenes de visualizaciones para dos cuerpos (cuadrado (figura 2.30) y triángulo (figura 2.31)) a diferente velocidad de la corriente libre y de giro (giro en sentido contrario a las agujas del reloj).

Cabe destacar que los patrones que se observan en las fotografías se mantienen estacionarios para cada posición de los cuerpos. La nitidez de la imagen fotográfica está relacionada con la repetitividad del flujo.

Figura 3.30 Visualización de flujo para el cuadrado 


\subsection{CORRECCIÓN POR BLOQUEO}

\subsubsection{Introducción}

Los resultados de los ensayos presentados en el punto 2.5.1.3 se han obtenido sin tener en cuenta los posibles efectos de bloqueo del túnel de viento.

El flujo que rodea un cuerpo inmerso en una corriente de aire delimitada por paredes rígidas es sujeto a una restricción u obstrucción. Los límites rígidos impiden un desplazamiento lateral libre del flujo de aire por el cuerpo, por lo que las velocidades alrededor del cuerpo son más altas de lo que serían si no existiesen dichas paredes.

Se puede determinar que dicho efecto es el producido por la relación de áreas entre el objeto a ensayar y la sección de pruebas del túnel de viento (figura 2.32). Dependiendo de tal relación se deberán corregir las mediciones mediante la utilización de coeficientes apropiados.

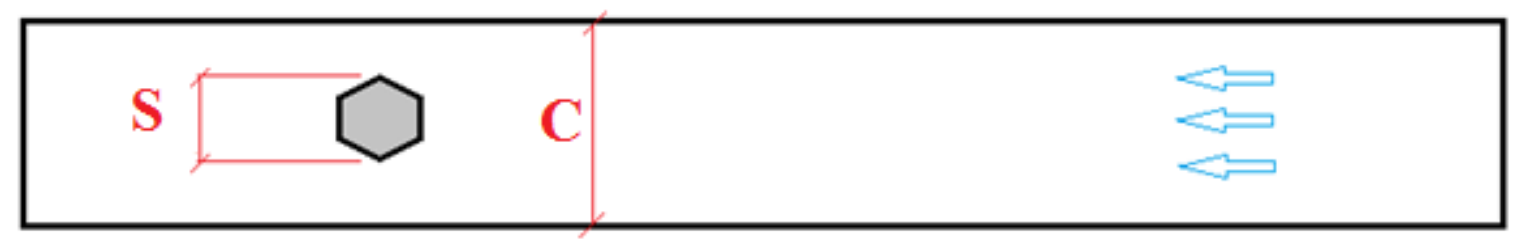

Figura 3.32 Relación de áreas entre objeto y túnel de viento.

En el presente apartado se realizan dos tipos de correcciones del efecto de bloqueo, una teórica a partir de la utilización de publicaciones y otra experimental, a partir de un desarrollo propio.

\subsubsection{Desarrollo}

\subsubsection{Geometrías}

Se considera que todos los cuerpos ensayados poseen las siguientes dimensiones:

- $h: 0,18 \mathrm{~m}$

- $\phi_{\text {mayor: }}: 0,035 \mathrm{~m}$

- Superficie del cuerpo, $S: 0,0063 \mathrm{~m}^{2}$

Nota: para los fines del cálculo se considera un diámetro promedio de todos los cuerpos. 
En cuanto al túnel de viento, posee las siguientes dimensiones:

- Alto del túnel, $\mathrm{h}_{\text {túnel }}: 0,2 \mathrm{~m}$

- Ancho del túnel, A: 0,2 m

- Área del túnel, C: 0,04 $\mathrm{m}^{2}$

Por lo tanto, se calcula que el cuerpo ocupa el 15,75\% de la sección de pruebas del túnel de viento y por tal, se puede considerar que ocurre efecto de bloqueo.

\subsubsection{Correcciones teóricas}

Para el análisis teórico se realizó un estudio de la bibliografía relacionada a la temática, del cual se pueden nombrar las publicaciones Wind Tunnel Testing [63], Wind Tunnel Wall Correction [64], Wind Tunnel Interference on Wings, Bodies and Airscrews [65], Wind tunnel blockage corrections: Review and application to Savonius vertical-axis wind turbines [66] y Wind Tunnel Tests on a Savonius Rotor [67].

Para el presente análisis se utilizaron las expresiones de Ross [66] y Alexander [67]. Ambos autores plantean una corrección en la velocidad incidente en el cuerpo, a partir de la siguiente expresión:

$\frac{V_{C}^{2}}{v^{2}}=\frac{1}{1-m \frac{S}{C}}$

donde,

- $V_{c}$ : velocidad corregida,

- $v$ : velocidad medida,

- $S$ : superficie frontal del cuerpo,

- C : área de la sección del túnel,

- $m$ : coeficiente que se determina a partir de la figura 2.33 . 

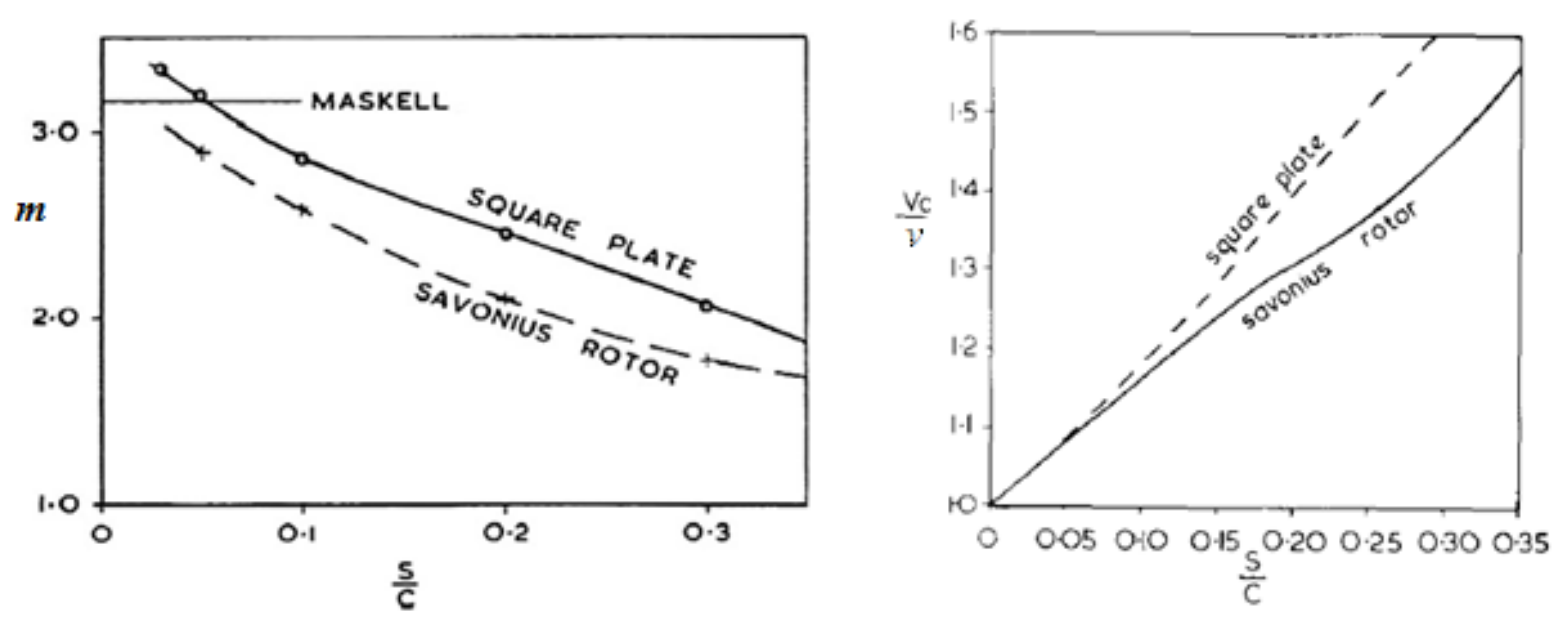

Figura 3.33 Variación de $m$ y de $V_{C} / v$ en función de la relación $S / C$ [66] [67].

Considerando:

- $S=0,0063 \mathrm{~m}^{2}$

- $\mathrm{C}=0,04 \mathrm{~m}^{2}$

- $m=2,25$

Se determina que la relación de velocidades es de 1,24. Por lo tanto, aplicando los criterios teóricos mencionados, se concluye que para corregir los valores de velocidad medidos, los mismos deben ser multiplicados por dicho valor constante.

\subsubsection{Correcciones experimentales}

Como verificación experimental, se realizó un ensayo similar a los desarrollados en el punto 2.5 en un túnel de viento grande (túnel TG) con una sección de prueba de $1,4 \mathrm{~m}^{2}$.

Para mantener el efecto bidimensional se dotó al sistema de un piso y techo postizos que poseen la misma separación en altura que el túnel chico utilizado para los ensayos (túnel TC), pero de $0,7 \mathrm{~m}$ de ancho. De esta manera la relación de bloqueo con el cuerpo a ensayar es de $4,5 \%$, aunque esta es menor, ya que queda espacio libre desde este sistema hasta las paredes del túnel TG (ver figuras 2.34, 2.35 y 2.36).

Se considera en este ensayo que el efecto del bloqueo es despreciable. 


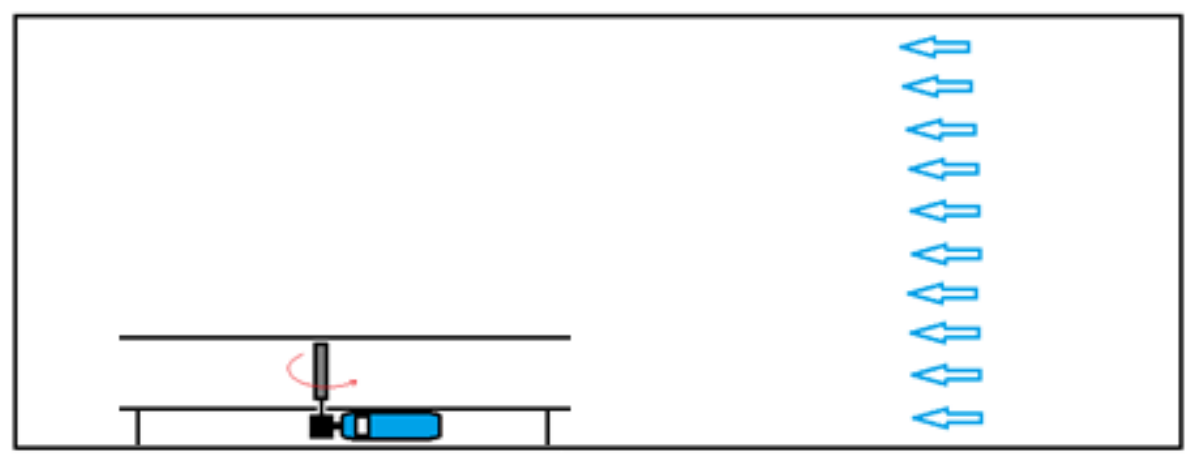

Figura 3.34 Vista lateral del ensayo en túnel de viento grande (túnel TG) para comparar efectos de bloqueo.

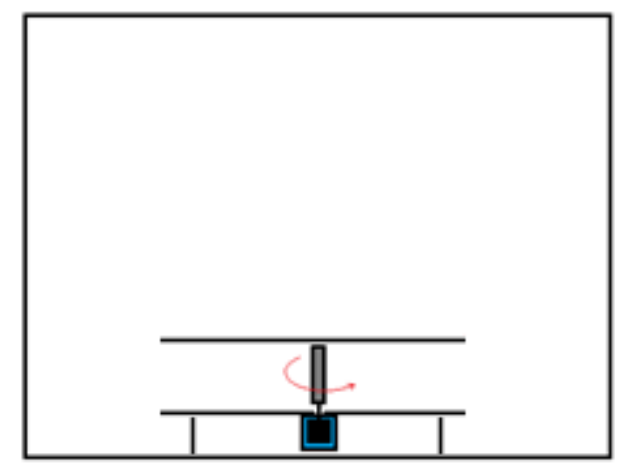

Figura 3.35 Vista frontal del ensayo en túnel de viento grande (túnel TG) para comparar efectos de bloqueo.

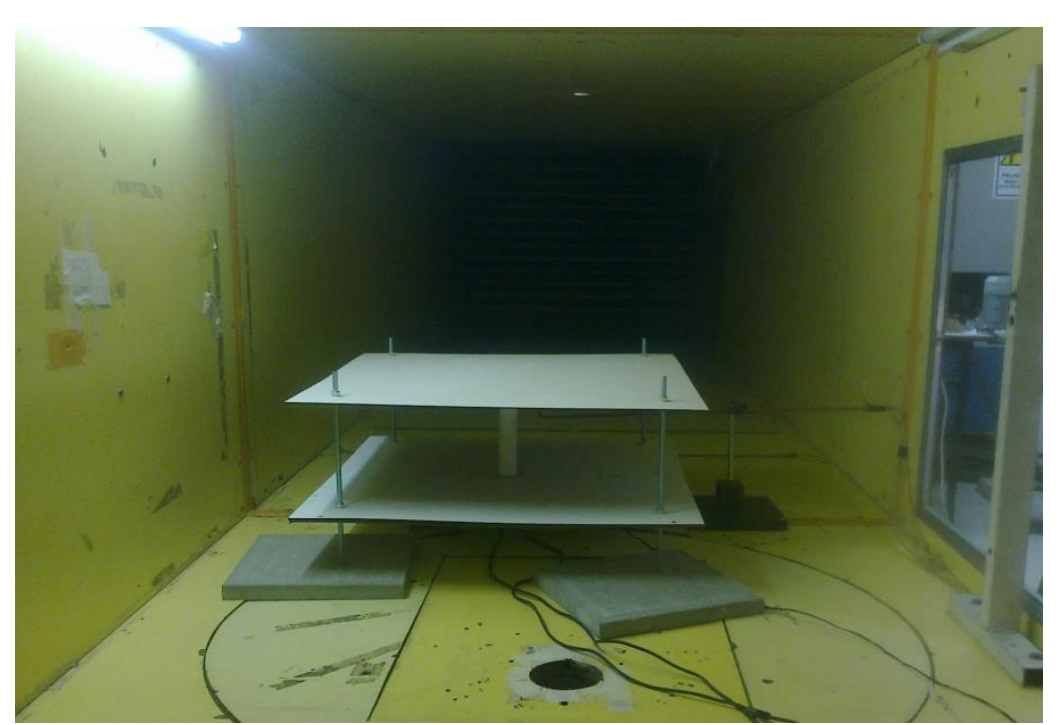

Figura 3.36 Montaje dentro del túnel de viento grande (túnel TG).

Se realizó el ensayo correspondiente a un cilindro, ya que es el modelo de referencia a utilizar. Se aplicó la siguiente metodología: 
En el ensayo dentro del túnel grande TG se realizó el mismo procedimiento de ensayo a velocidades de giro constantes y haciendo un barrido de velocidades que se realizó en el túnel chico TC. Se ensayó solo para $1950 \mathrm{rpm}$.

Cabe destacar que para el ensayo dentro del túnel grande TG se realizaron tres corridas para cada velocidad de la corriente y se calculó el promedio de cada sustentación. De esta manera se ajustaron los valores reduciendo el error de la medición. En la tabla 2.12 se presentan los resultados de los ensayos de bloqueo en el túnel grande TG y los correspondientes al ensayo realizado en el túnel chico $\mathrm{TC}$.

Tabla 2.12 Resultados correspondientes a los ensayos de medición de sustentación $L$ en ambos túneles (TG y TC) para la misma velocidad de la corriente libre $v$.

\begin{tabular}{|c|c|c|c|}
\hline $\begin{array}{c}\omega \\
{[\mathbf{r p m}]}\end{array}$ & $\begin{array}{c}\boldsymbol{v} \\
{[\mathbf{m} / \mathbf{s}]}\end{array}$ & $\begin{array}{c}\boldsymbol{L} \\
\text { Túnel } \boldsymbol{T} \boldsymbol{G} \\
{[\mathbf{N}]}\end{array}$ & $\begin{array}{c}\boldsymbol{L} \\
\text { Túnel } \boldsymbol{T C} \\
{[\mathbf{N}]}\end{array}$ \\
\hline \multirow{5}{*}{1950} & 0,00 & 0,00 & 0,00 \\
\cline { 2 - 4 } & 5,30 & 0,06 & 0,08 \\
\cline { 2 - 4 } & 7,98 & 0,07 & 0,10 \\
\cline { 2 - 4 } & 10,41 & 0,07 & 0,12 \\
\cline { 2 - 4 } & 11,54 & 0,08 & 0,14 \\
\hline
\end{tabular}

Como se considera que en el túnel grande TG no existe el efecto de bloqueo, se procede a determinar la relación entre el coeficiente de sustentación $C_{L}$ y la velocidad de la corriente $v$, para cada velocidad de giro de acuerdo a la ecuación (6).

$L_{T G}=\frac{1}{2} \rho v^{2} S C_{L}$

En base a los resultados obtenidos en la mencionada relación se realizó un ajuste cuadrático (figura 2.37). 


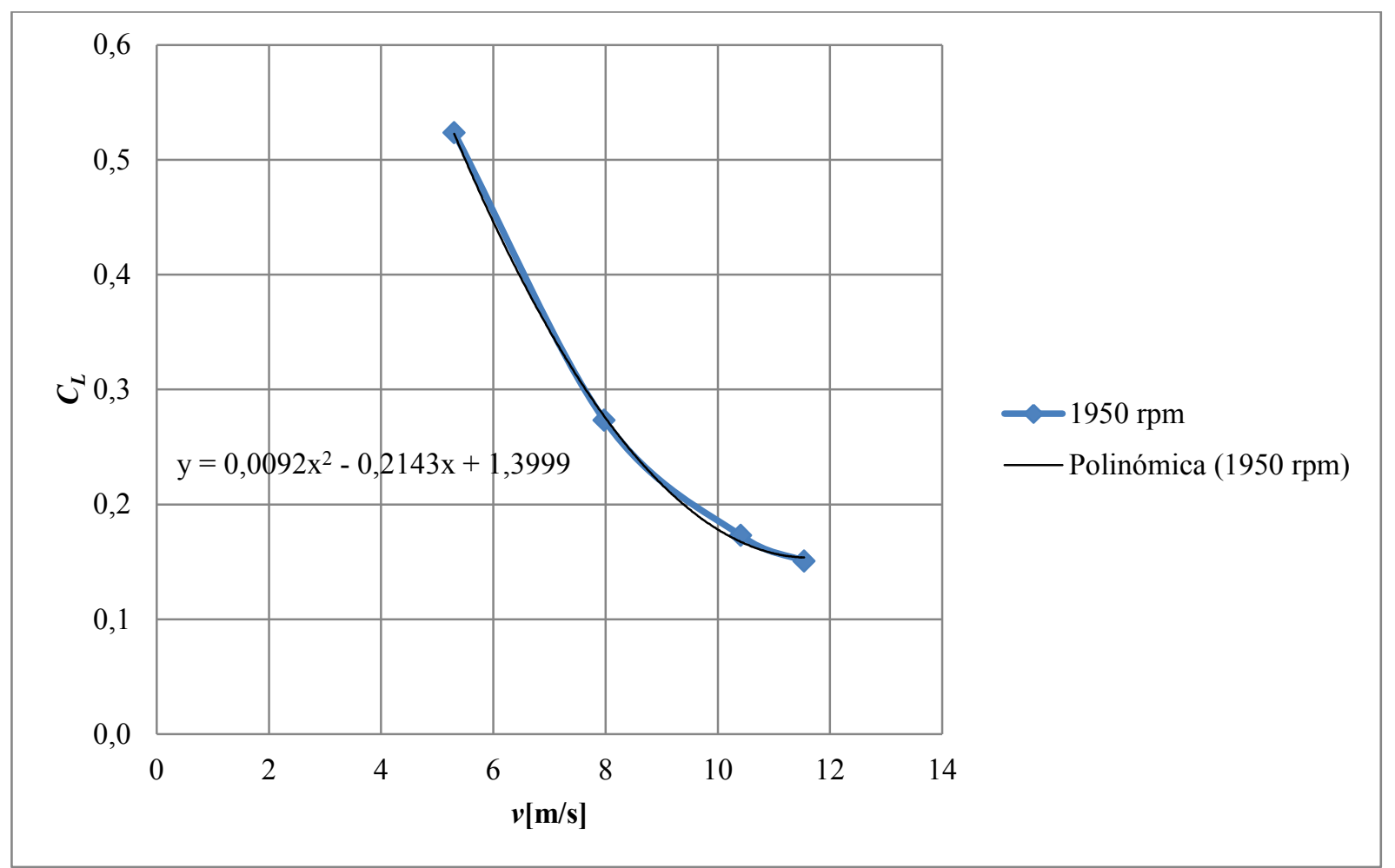

Figura 3.37 Relación y ajuste del coeficiente de sustentación $C_{L}$ con la velocidad de la corriente $v$, para el ensayo en el túnel (TG).

Se asume como criterio que la variación de $C_{L}$ con la velocidad de la corriente para cada velocidad de rotación es la misma para ambos túneles. Considerando la sustentación en el túnel chico TC en función de la velocidad corregida $V_{C}$ se plantea la ecuación (7).

$L_{T C}=\frac{1}{2} \rho V_{C}^{2} S C_{L}$

Reemplazando el ajuste determinado en la figura 2.37, con el objetivo de determinar $V_{C}$, se determina la siguiente ecuación:

Para $1950 \mathrm{rpm}: \quad L_{T C}=\frac{1}{2} \rho S\left(0,0092 V_{C}^{4}-0,2143 V_{C}^{3}+1,3999 V_{C}^{2}\right)$

Se obtienen los resultados presentados en la tabla 2.13 . 
Tabla 2.13 Relación entre velocidad corregida y velocidad medida en el túnel chico TC.

\begin{tabular}{|c|c|c|c|}
\hline $\begin{array}{c}\omega \\
{[\mathbf{r p m}]}\end{array}$ & $\begin{array}{c}\boldsymbol{v} \\
{[\mathbf{m} / \mathbf{s}]}\end{array}$ & $\begin{array}{c}\boldsymbol{V}_{\boldsymbol{C}} \\
{[\mathbf{m} / \mathbf{s}]}\end{array}$ & $\boldsymbol{V}_{\boldsymbol{C}} / \boldsymbol{v}$ \\
\hline \multirow{4}{*}{1950} & 0,00 & 0,00 & - \\
\cline { 2 - 4 } & 5,30 & 11,84 & 2,23 \\
\cline { 2 - 4 } & 7,98 & 12,75 & 1,60 \\
\cline { 2 - 4 } & 10,41 & 13,35 & 1,28 \\
\cline { 2 - 4 } & 11,54 & 13,83 & 1,20 \\
\hline
\end{tabular}

Se concluye que, a diferencia de la corrección teórica, la relación de velocidades no es constante y varía tanto con la velocidad angular como con la velocidad de la corriente libre. Así mismo, cabe destacar que el factor determinado en la corrección teórica se encuentra dentro de intervalo de los hallados experimentalmente.

Así mismo, estas hipótesis se pueden validar observando los resultados. Se observa que hay un valor de sustentación que coincide para ambos túneles, que es de $0,08 \mathrm{~N}$, para 5,30 m/s en el túnel chico TC y 11,54 m/s en el túnel grande TG (tabla 2.12). Si analizamos las correcciones de la tabla 2.13 podemos ver que la relación de velocidad para $5,30 \mathrm{~m} / \mathrm{s}$ es de 2,23, lo que da una velocidad corregida de $11,84 \mathrm{~m} / \mathrm{s}$, validando así el criterio propuesto.

\subsubsection{Conclusiones bloqueo}

Puede observarse una disminución en la sustentación en el túnel grande respecto al chico para una misma velocidad de la corriente de referencia, ya que, como era de esperar, la velocidad de la corriente en torno al cuerpo ensayado disminuye al no existir paredes en la cercanía del mismo.

Tanto las correcciones teóricas como experimentales presentan una relación de velocidades entre la medida y la corregida que permitiría corregir los valores de los ensayos experimentales descriptos en el punto 2.5.1. El valor de corrección por bloqueo teórico coincide con el experimental para velocidades de la corriente del túnel chico en el entorno de $10 \mathrm{~m} / \mathrm{s}$.

Para la presente tesis estos valores serán utilizados con el fin de corregir sólo los resultados del cilindro y de esta manera poder compararlo con las publicaciones correspondientes. 
Las mismas no serán utilizadas para el resto de los cuerpos, ya que como los resultados son con fines comparativos, no es necesario afectarlos por tal corrección y requeriría de tiempo y más ensayos que escapan al presente estudio.

\subsubsection{Comparación de resultados con modelos de la bibliografía}

Con el fin de validar los ensayos y el método, se comparan a continuación los resultados de los ensayos corregidos con los de la bibliografía, para el caso del cilindro circular liso.

Para el caso del cilindro liso, se eligió un factor único para corregir la velocidad de la corriente, que es el promedio de las correcciones en el rango de velocidades de la corriente ensayado para $\omega=1950 \mathrm{rpm}$, y se determina a partir del ajuste de la figura 2.37; con esto se corrigieron el coeficiente de sustentación $C_{L}$ y la relación de velocidades $u / v$.

Se utilizan las publicaciones de Prandtl, L. [3], Ackeret, J. [68], Reid, E. C. [69] y Badalamenti, C. [72] donde se presentan curvas de la variación del $C_{L}$ en función de $u / v$ de ensayos experimentales de cuerpos cilíndricos circulares.

En el caso de Prandtl [3] y Ackeret [68] los ensayos son realizados en un cilindro circular utilizando tapas de diferente diámetro en sus extremos para hacer un estudio bidimensional. El cuerpo posee un largo de 0,3 m y 0,07 $\mathrm{m}$ de ancho y las tapas de 0,12 y 0,14 m de diámetro. Los ensayos se realizaron a valores de números de Reynolds entre: $38000<\mathrm{R}_{\mathrm{e}}<320000$.

Por otro lado, en el caso de Reid [69] también realiza ensayos en un cilindro circular pero que ocupa el total del ancho del túnel de viento, por lo que se consideran también ensayos bidimensionales. El cuerpo posee una superficie frontal de $0,1741 \mathrm{~m}^{2}$ y los ensayos se realizaron a valores de números de Reynolds entre: $43000<\mathrm{R}_{\mathrm{e}}<128000$.

Finalmente, Badalamenti [72] publica unos gráficos realizados por Takayama [73] en los cuales se representan ensayos realizados sobre cilindros lisos y con rugosidad, con tapas en sus extremos y con un alargamiento de 2,15. Dichos ensayos se realizaron a valores de números de Reynolds entre $40000 \leq \mathrm{R}_{\mathrm{e}} \leq 180000$.

A continuación se presenta en la figura 2.38 los resultados superpuestos de ambas publicaciones para un cuerpo cilíndrico circular y del correspondiente a los ensayos experimentales corregidos por bloqueo. 


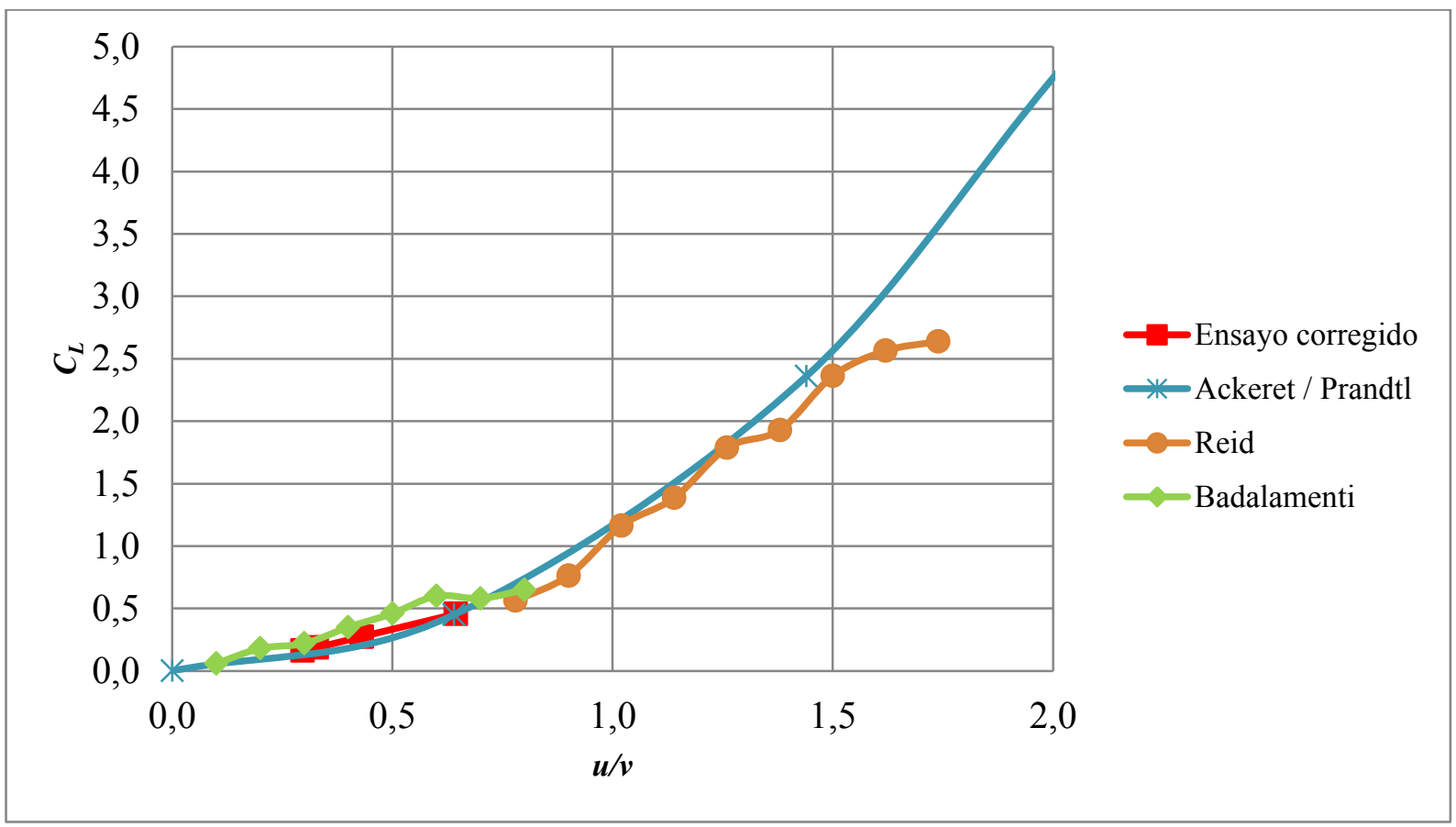

Figura 3.38 Variación del coeficiente de sustentación $C_{L}$ en función de la relación de velocidades $u / v$ de ensayos experimentales, Ackeret [68], Reid [69] y Badalamenti [72].

Puede observarse en el rango de $u / v$ correspondiente a los ensayos de esta tesis que el primer punto de los resultados de los ensayos de Ackeret/Prandtl $\left(u / v=0,46, C_{L}=0,64\right)$ coincide con el obtenido en esta tesis; mientras que los resultados publicados por Badalamenti difieren en el orden del $20 \%$ en el valor de $C_{L}$ con los obtenidos en la tesis. Cabe señalar que Takayama realizó los ensayos en un modelo con un alargamiento y geometría del modelo diferente al de la presente tesis.

A partir de esto, se considera validada la metodología de ensayo de la presente tesis. 


\section{CAPITULO 3}

\section{ANÁLISIS DE RESULTADOS}

\subsection{INTRODUCCIÓN}

Con el fin de caracterizar el flujo alrededor de los cuerpos y entender su comportamiento aerodinámico se realiza un análisis específico a partir de los datos obtenidos en los ensayos, descriptos en el Capítulo 2.

Se analizan las visualizaciones y por medio de las mismas se estudia el desarrollo del flujo en el entorno de los cuerpos, lo que permite describir las variaciones en las fuerzas generadas.

\subsection{DESARROLLO}

Para el análisis de los flujos de los diversos cuerpos se utilizan las curvas de variación de los coeficientes aerodinámicos $C_{L}$ y $C_{D}$ en función de la relación de velocidades $u / v$. Como predice el análisis dimensional, en la mayoría de los cuerpos dicha variación se superpone para las diferentes velocidades de giro $\omega$ ensayadas y por lo tanto se toma una única curva para cada cuerpo que representa el comportamiento de dicha variación (curva característica). Excepcionalmente en algunos casos esto no ocurre (aparece más de una curva característica) y en esos casos se estudian los diferentes comportamientos a diferentes velocidades de giro $\omega$.

La existencia de las curvas características permite comparar el comportamiento de los diversos cuerpos dando como resultado que se puedan agrupar los cuerpos en diferentes familias. En base a las visualizaciones mencionadas en el apartado 2.5.2 se puede determinar el comportamiento del patrón aerodinámico de cada cuerpo y así inferir una explicación a la respuesta de cada cuerpo ante el efecto Magnus. 
En las figuras de las visualizaciones se presentan fotografías del flujo junto a un esquema con las principales características del flujo (desprendimientos, torbellinos, líneas de corriente) para facilitar la explicación. A veces la interpretación de las fotografías no es sencilla, y ha sido realizada a partir de las secuencias de video construidas a partir de las fotografías, ya que los procesos dinámicos facilitan el reconocimiento de patrones.

Se presentan las diversas familias no por el orden numérico de los resultados, sino por el más conveniente para la discusión, dejando la familia $\mathrm{V}$ correspondiente a los cuerpos circulares al final, ya que en las visualizaciones no se pueden sincronizar los torbellinos y por tal no son base para la discusión de las conclusiones. 


\subsubsection{Familia I}

La misma está conformada por el decágono, octógono y hexágono. En las figuras 3.1 y 3.2 se observa la variación de los coeficientes aerodinámicos $C_{L}$ y $C_{D}$ de los mencionados cuerpos con la relación de velocidades $u / v$. Los puntos $\mathrm{R}$ señalados en las mismas corresponden a diferentes relaciones de $u / v$ para las que se tomaron fotografías con el fin de analizar el flujo correspondiente.

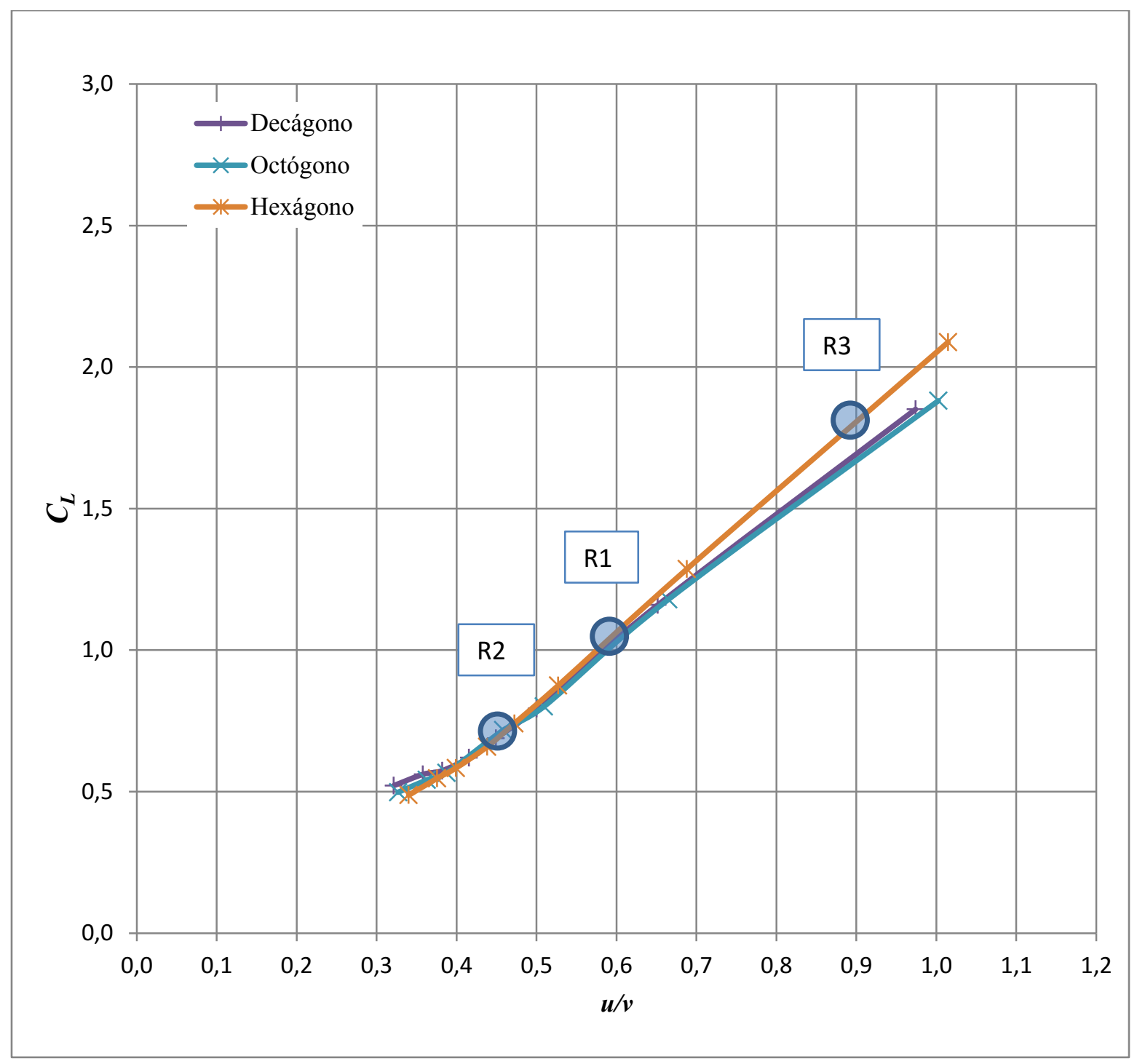

Figura 3.1 Variación del coeficiente de sustentación $C_{L}$ en función de la relación de velocidades $u / v$, relaciones R1, R2 y R3 para el análisis fotográfico (círculos gruesos). 
Del hexágono se tomaron fotografías en tres condiciones $\mathrm{R} 1(u / v=0,60), \mathrm{R} 2(u / v=0,47) \mathrm{y}$ $\mathrm{R} 3(u / v=0,90)$, en tanto que en los cuerpos restantes sólo en $\mathrm{R} 1$, ya que la diferencia entre los patrones de flujo para esos cuerpos, para las otras relaciones de $u / v$, es semejante a la que hay en R1.

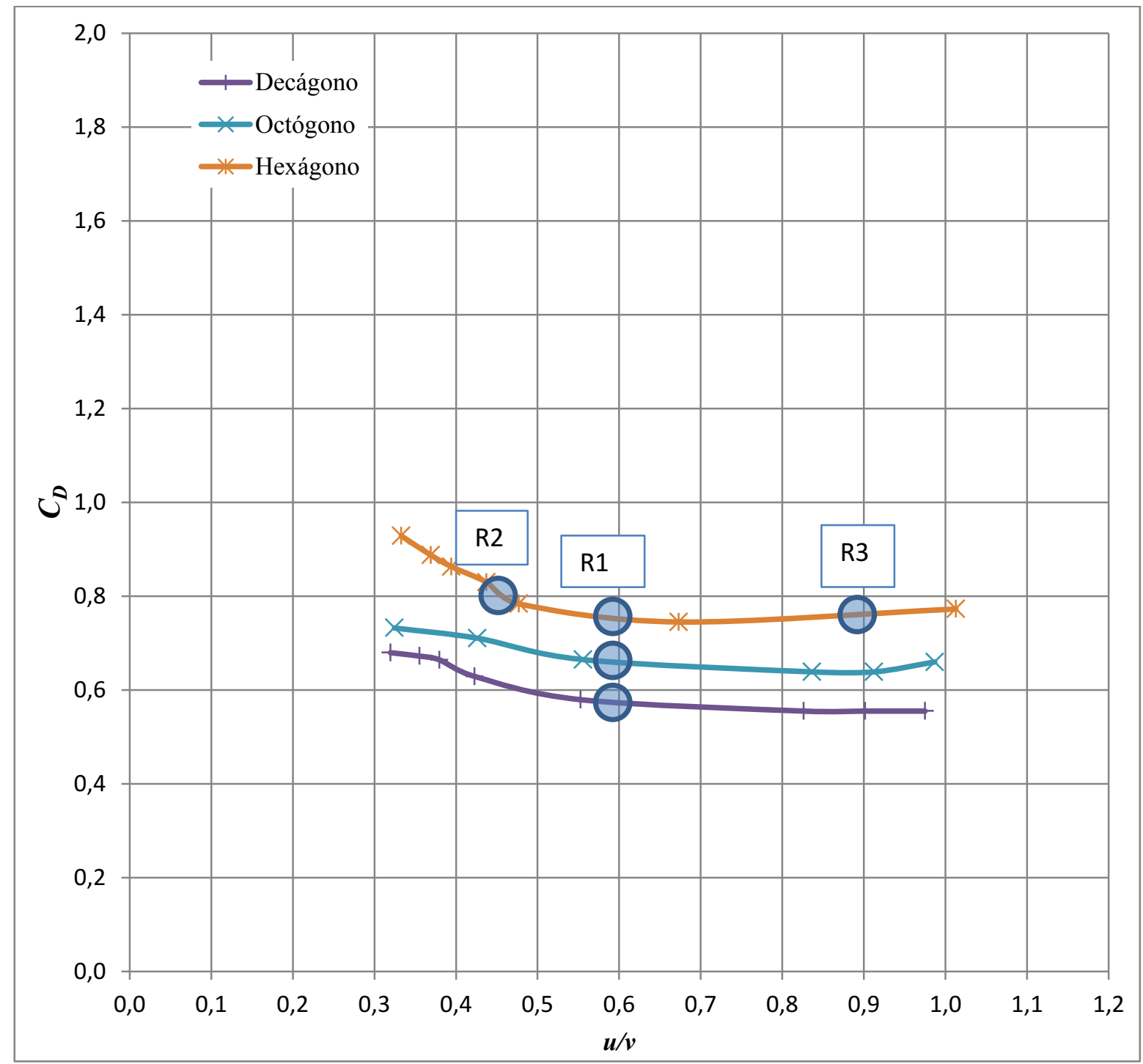

Figura 3.2 Variación del coeficiente de sustentación $C_{D}$ en función de la relación de velocidades $u / v$ y relación R1, R2 y R3 para el análisis fotográfico (círculos gruesos).

A continuación se realiza una descripción de los flujos correspondientes a los diversos cuerpos de la Familia I para las relaciones R1, R2 y R3. 


\section{R1 - Relación $u / v=0,60(\omega=2520 \mathrm{rpm}, v=7,7 \mathrm{~m} / \mathrm{s})$}

En la figura 3.3 se observa el comportamiento del flujo para el decágono.
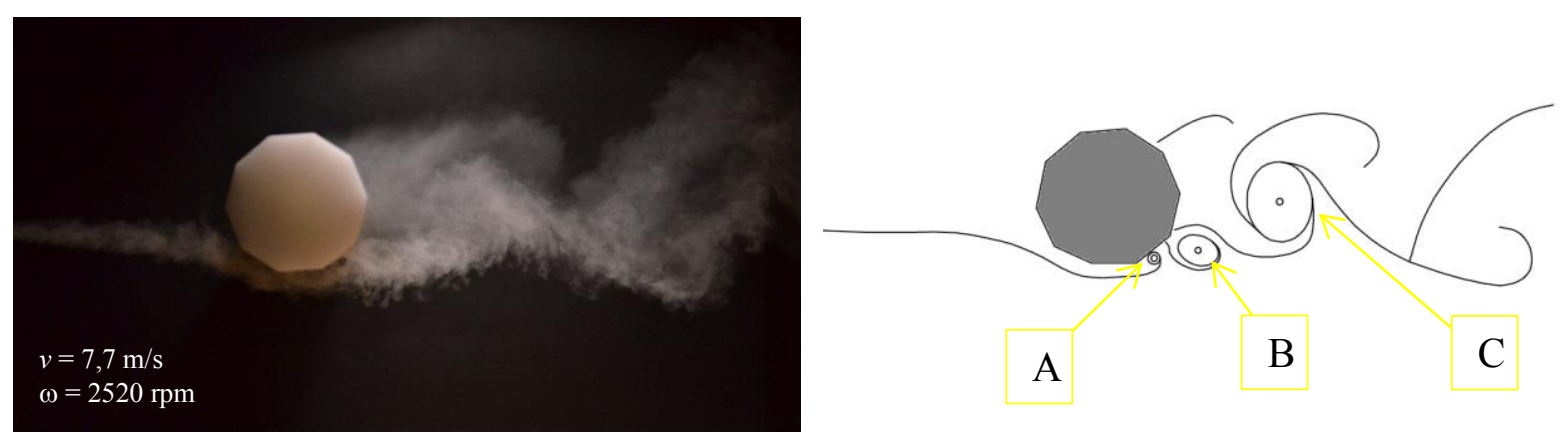

Figura 3.3 Configuración de flujo entorno al cuerpo.

En la arista inferior del decágono se produce el desprendimiento del flujo, lo que genera el torbellino $A$, que en el paso siguiente $\left(\theta=18^{\circ}\right.$ no representada aquí) avanza hasta la posición $\mathrm{B}$ y luego en el paso siguiente $\left(\theta=36^{\circ}\right.$ equivalente a $\left.\theta=0^{\circ}\right)$ toma la posición $\mathrm{C}$. Esta secuencia de torbellinos se genera de igual forma ciclo a ciclo.

En la figura 3.4 se observa el comportamiento del flujo para el octógono en diversas fases de giro.
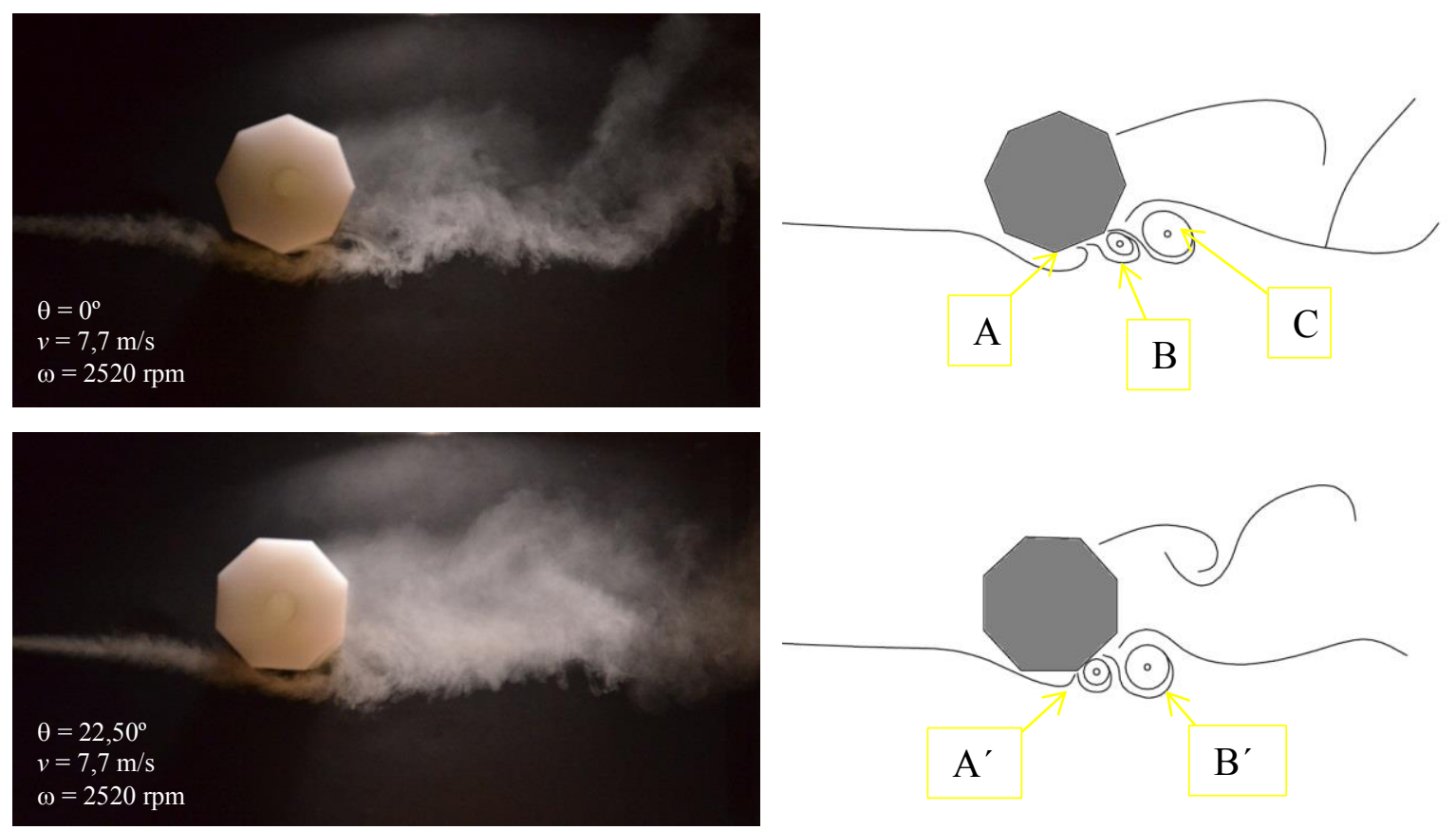

Figura 3.4 Configuración de flujo entorno al cuerpo en cada posición $\theta$. 
$\theta=0^{\circ}$ : se genera un desprendimiento A debido a la arista aguda, y se observa el torbellino B generado en el paso de la arista anterior.

El torbellino $\mathrm{C}$ corresponde al torbellino $\mathrm{B}^{\prime}$ de la fase anterior del giro del cuerpo $\left(\theta=0^{\circ}\right.$ que coincide $\operatorname{con} \theta=45^{\circ}$ ).

$\theta=22,5^{\circ}$ : el torbellino $\mathrm{A}$ se desplaza hasta $\mathrm{A}^{\prime} \mathrm{y}$ el torbellino $\mathrm{B}$ hasta $\mathrm{B}^{\prime}$, en tanto que el torbellino $\mathrm{C}$ se disuelve en la estela turbulenta.

En la figura 3.5 se observa el comportamiento del flujo para el hexágono.
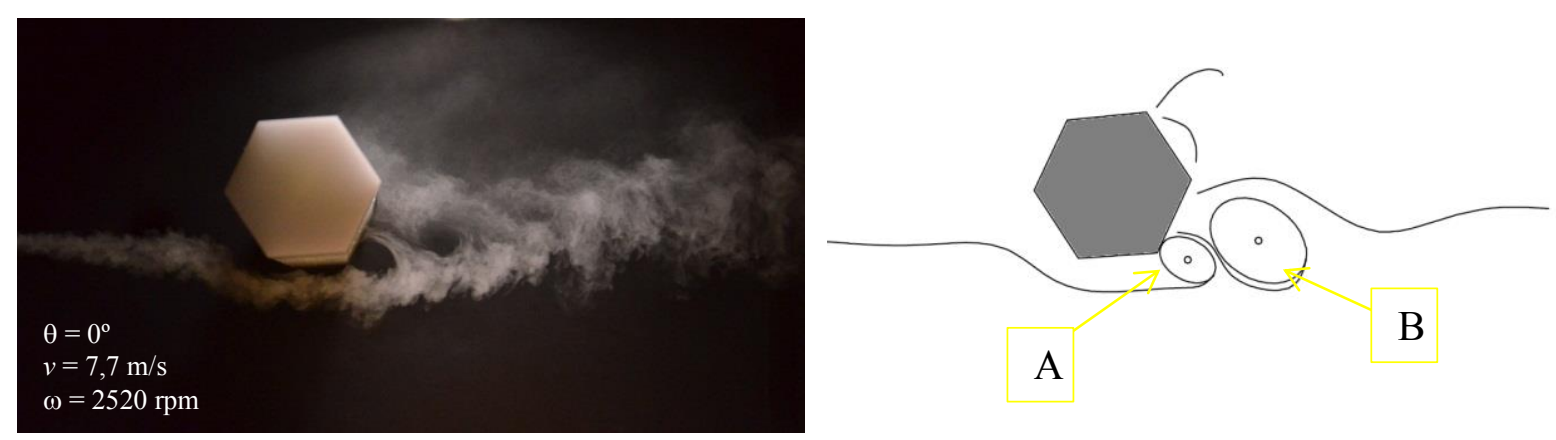

A
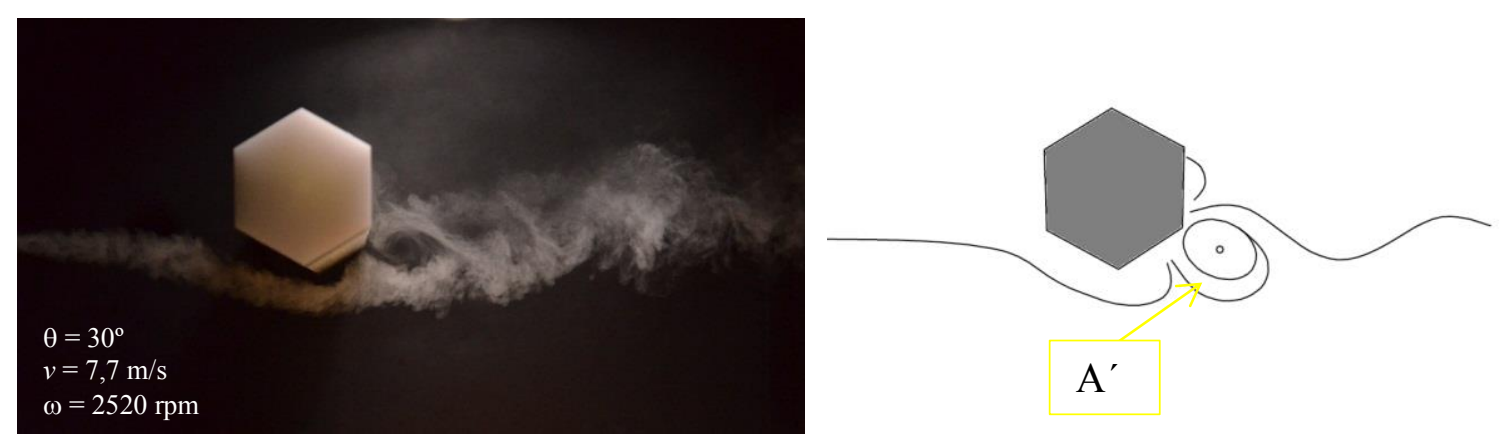

Figura 3.5 Configuración de flujo entorno al cuerpo en cada posición $\theta$.

$\theta=0^{\circ}$ : el desprendimiento en la arista inferior $\left(\operatorname{ver} \theta=30^{\circ}\right.$ ) genera el comienzo del torbellino A, que se desarrollará hasta pasar a ser el torbellino B.

$\theta=30^{\circ}$ : el torbellino A está completamente desarrollado (en $\mathrm{A}^{\prime}$ ) y el torbellino $\mathrm{B}$ se ha disuelto en la estela turbulenta. 


\section{R2 - Relación $u / v=0,47(\omega=1950 \mathrm{rpm}, v=7,7 \mathrm{~m} / \mathrm{s})$ - sólo hexágono}

En la figura 3.6 se observa el comportamiento del flujo para el hexágono.
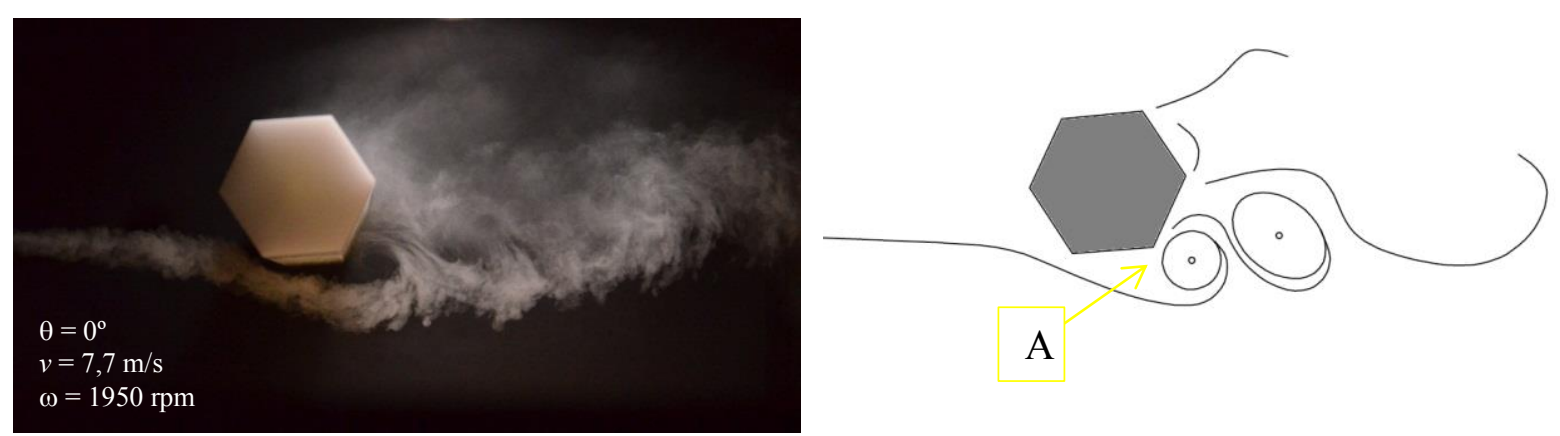

A
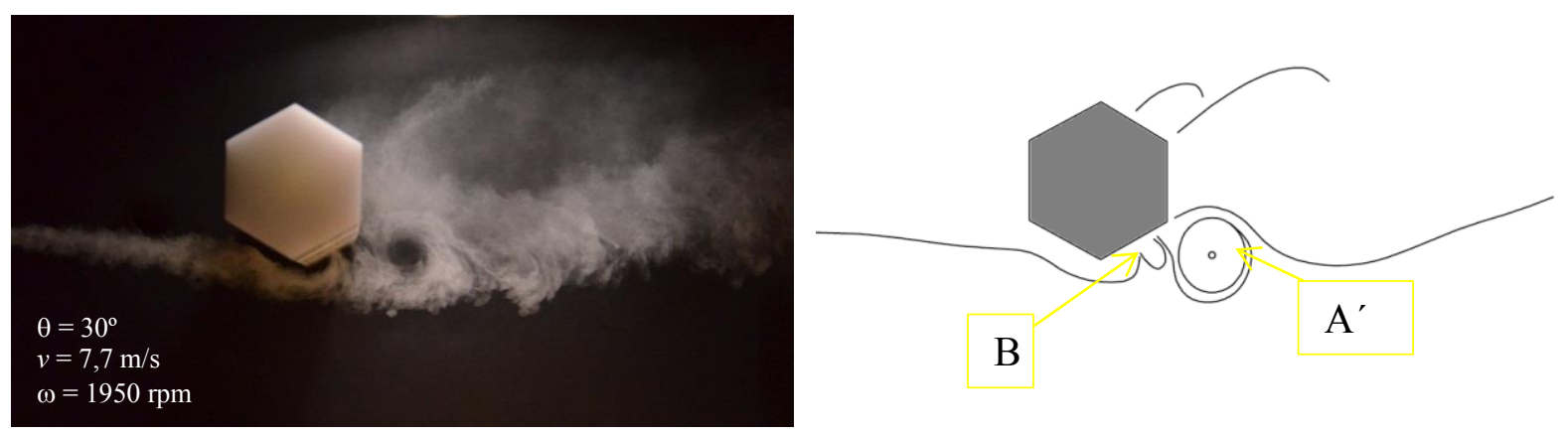

Figura 3.6 Configuración de flujo entorno al cuerpo en cada posición $\theta$.

$\theta=0^{\circ}$ : se genera el torbellino A en la arista afilada.

$\theta=30^{\circ}$ : el torbellino A (en $\mathrm{A}^{\prime}$ ) está completamente desarrollado y comenzando a separarse del cuerpo.

El desprendimiento de flujo B por la arista inferior $\left(\theta=30^{\circ}\right)$ genera el torbellino A (en $\theta=60^{\circ}$, o $\theta=0^{\circ}$ ).

Hay un flujo remansado en la parte superior trasera del cuerpo. 


\section{R3 - Relación $u / v=0,90(\omega=2520 \mathrm{rpm}, v=5,2 \mathrm{~m} / \mathrm{s})$ - sólo hexágono}

En la figura 3.7 se observa el comportamiento del flujo para el hexágono.
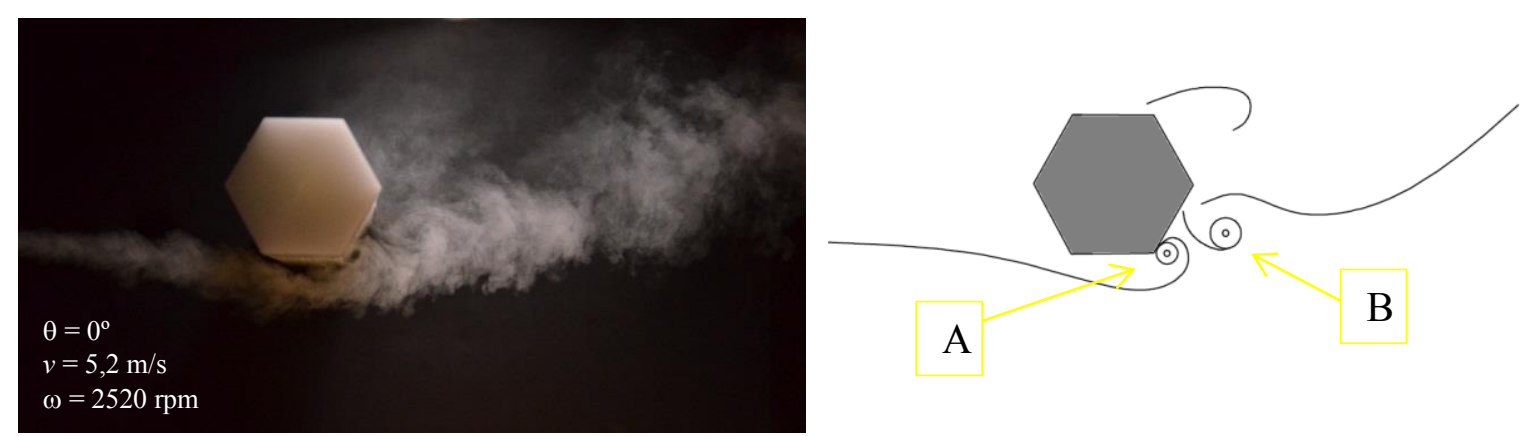

A
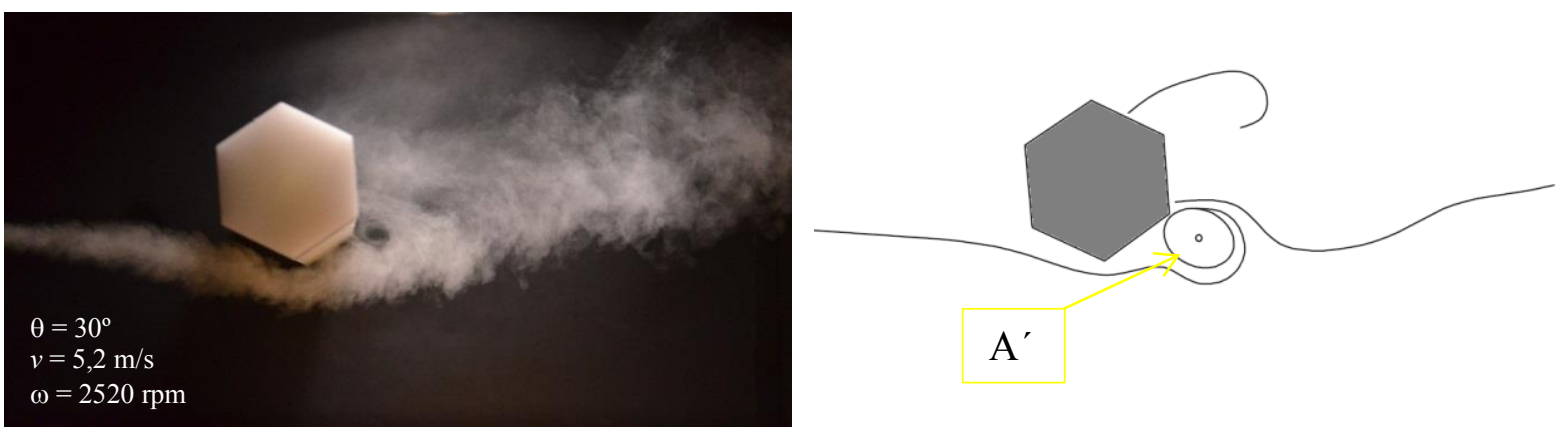

Figura 3.7 Configuración de flujo entorno al cuerpo en cada posición $\theta$.

$\theta=0^{\circ}$ : los torbellinos A y B se forman y se unen para desarrollarse en $\theta=30^{\circ}$ y generar el torbellino $\mathrm{A}^{\prime}$, que se desprende en la próxima fase.

$\theta=30^{\circ}$ : único torbellino $\mathrm{A}^{\prime}$ en la parte inferior trasera del cuerpo.

Flujo en remanso en la parte superior trasera del cuerpo.

\subsubsection{Conclusiones familia I}

El desprendimiento de torbellinos está sincronizado con el giro del cuerpo.

La variación de $C_{L}$ con $u / v$ (figura 3.1) es muy semejante dentro de la familia, mientras que la variación de $C_{D}$ está ordenada según el número de caras, es decir que a menor cantidad de caras, mayor resistencia. A su vez la relación $u / v$ no influye en el $C_{D}$ cuando $u / v>0,5$.

En el caso del decágono se observa un patrón muy similar al octógono, sólo que al tener más caras y por ende aristas menos filosas, se observa un pequeño torbellino adherido detrás de las mismas en la parte inferior del cuerpo, que es el generador de los torbellinos siguientes. 
Para el caso del octógono, pueden observarse dos torbellinos, uno por cada arista, en la parte inferior del cilindro. Así mismo, estos se van desprendiendo y continúan corriente abajo aumentando su diámetro. Todos los torbellinos mencionados (de la capa inferior de la estela) poseen sentido de giro anti horario.

En el hexágono, al igual que en los dos cuerpos anteriores, se genera un torbellino a causa de la arista. Como el cuerpo posee menor cantidad de caras, este torbellino es de mayor diámetro que los mencionados anteriormente.

El hecho de que el $C_{L}$ (para un cierto $u / v$ ) se mantenga prácticamente igual al cambiar el número de lados (dentro de esta familia) parece sugerir que aunque al aumentar el número de caras se aumenta la frecuencia de desprendimientos de torbellinos, la intensidad de los mismos disminuye, de forma que la intensidad total por unidad de tiempo permanece constante.

Así mismo, puede observarse que al aumentar la velocidad de la corriente manteniendo la velocidad de giro (disminuir $u / v$ ) se produce un aumento considerable en el tamaño del torbellino, mientras que si se mantiene la velocidad de la corriente y se aumenta la velocidad de giro (aumentar $u / v$ ), no se presentan cambios considerables en el patrón.

Producto de estos torbellinos puede explicarse la diferencia de los valores de $C_{D}$ para los diferentes cuerpos. Se observa que a menor cantidad de caras (tamaño más grande de los torbellinos) más altos son los valores de $C_{D}$. 


\subsubsection{Familia II}

La misma está conformada por el cuadrado, cuadrado de bordes redondeados y el circular con aletas que se asemeja a un cuerpo con cuatro aristas. En las figuras 3.8 y 3.9 se observa la variación de los coeficientes aerodinámicos $C_{L}$ y $C_{D}$ de los mencionados cuerpos con la relación de velocidades $u / v$. Los puntos $\mathrm{R}$ señalados en las mismas corresponden a diferentes relaciones de $u / v$ para las que se tomaron fotografías con el objeto de determinar el comportamiento del flujo alrededor de estos cuerpos.

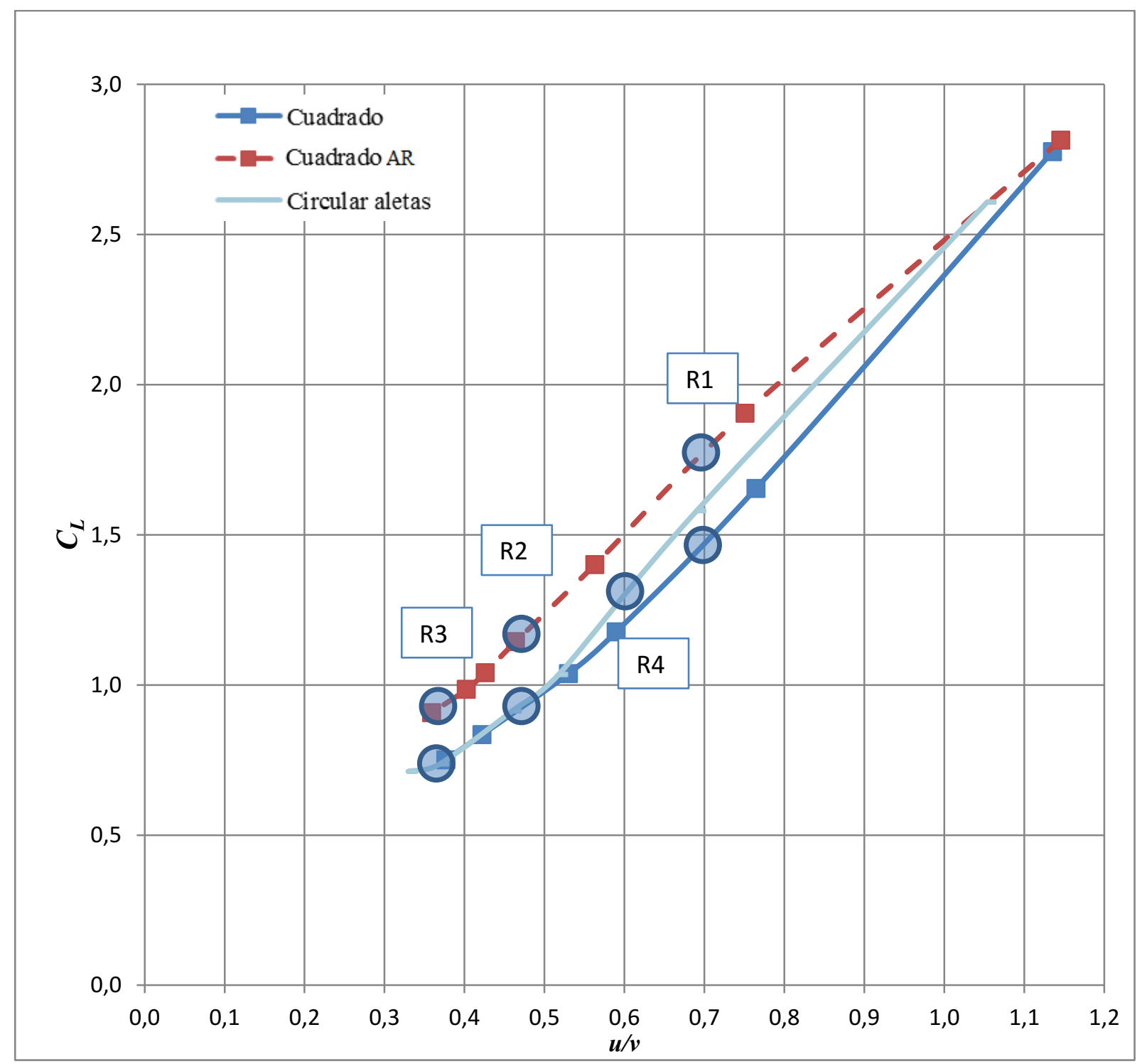

Figura 3.8 Variación del coeficiente de sustentación $C_{L}$ en función de la relación de velocidades $u / v$ y relaciones $\mathrm{R} 1, \mathrm{R} 2, \mathrm{R} 3 \mathrm{y} \mathrm{R} 4$ para el análisis fotográfico (círculos gruesos). 
Del cuerpo circular con aletas sólo se tomaron fotografías para la relación $\mathrm{R} 4(u / v=0,60)$, en tanto que a los cuerpos restantes se tomaron para tres relaciones $\mathrm{R} 1(u / v=0,70)$, $\mathrm{R} 2(u / v=0,47)$ y $\mathrm{R} 3(u / v=0,36)$.

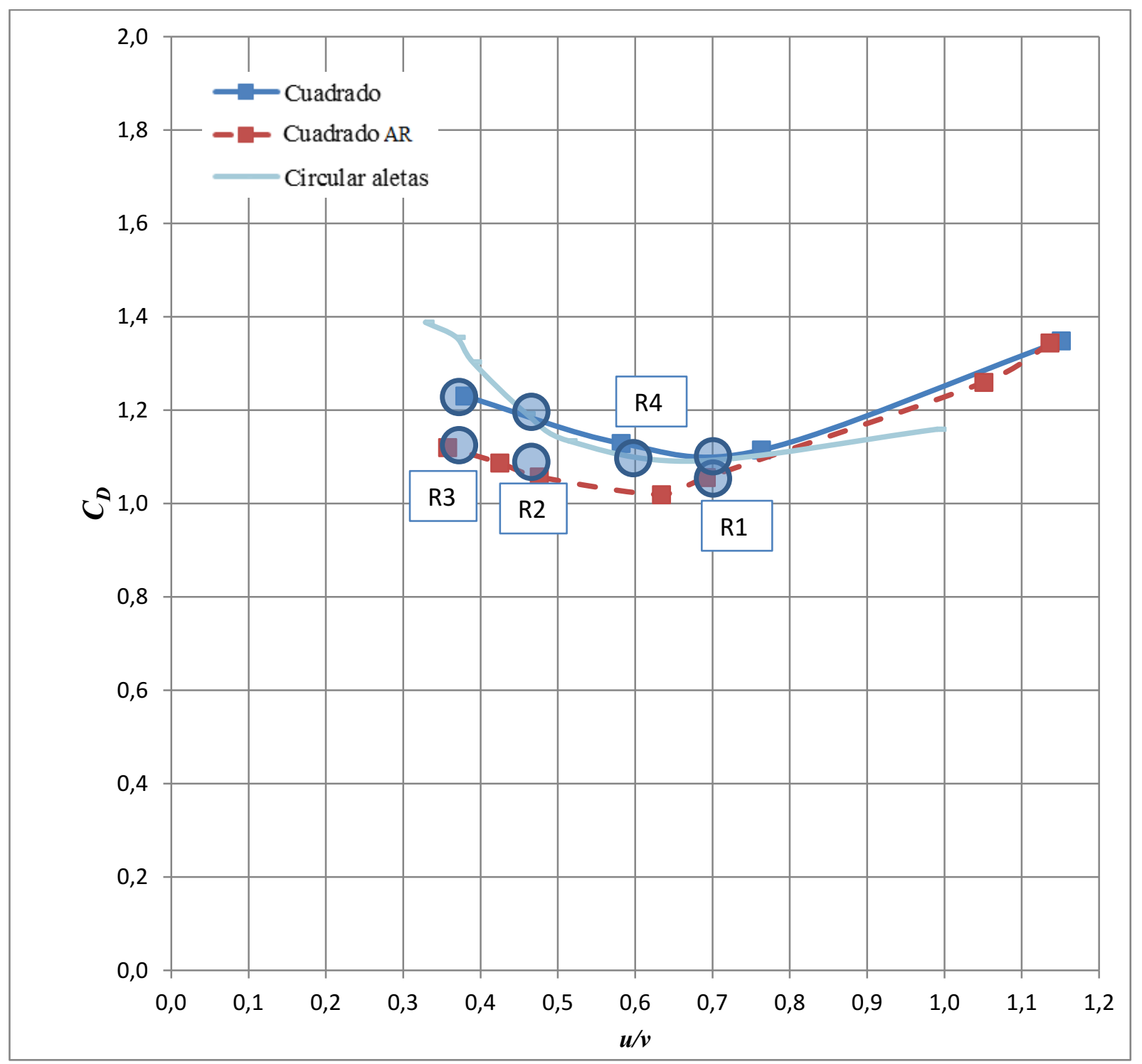

Figura 3.9 Variación del coeficiente de sustentación $C_{D}$ en función de la relación de velocidades $u / v$ y relaciones $\mathrm{R} 1, \mathrm{R} 2, \mathrm{R} 3$ y R4 para el análisis fotográfico (círculos gruesos).

A continuación se realiza una descripción de los flujos correspondientes a los diversos cuerpos de la Familia II para las relaciones R1, R2, R3 y R4. 


\section{$\mathrm{R} 1$ - Relación $u / v=0,70(\omega=1950 \mathrm{rpm}, v=5,2 \mathrm{~m} / \mathrm{s})$}

En la figura 3.10 se observa el comportamiento del flujo para el cuadrado.
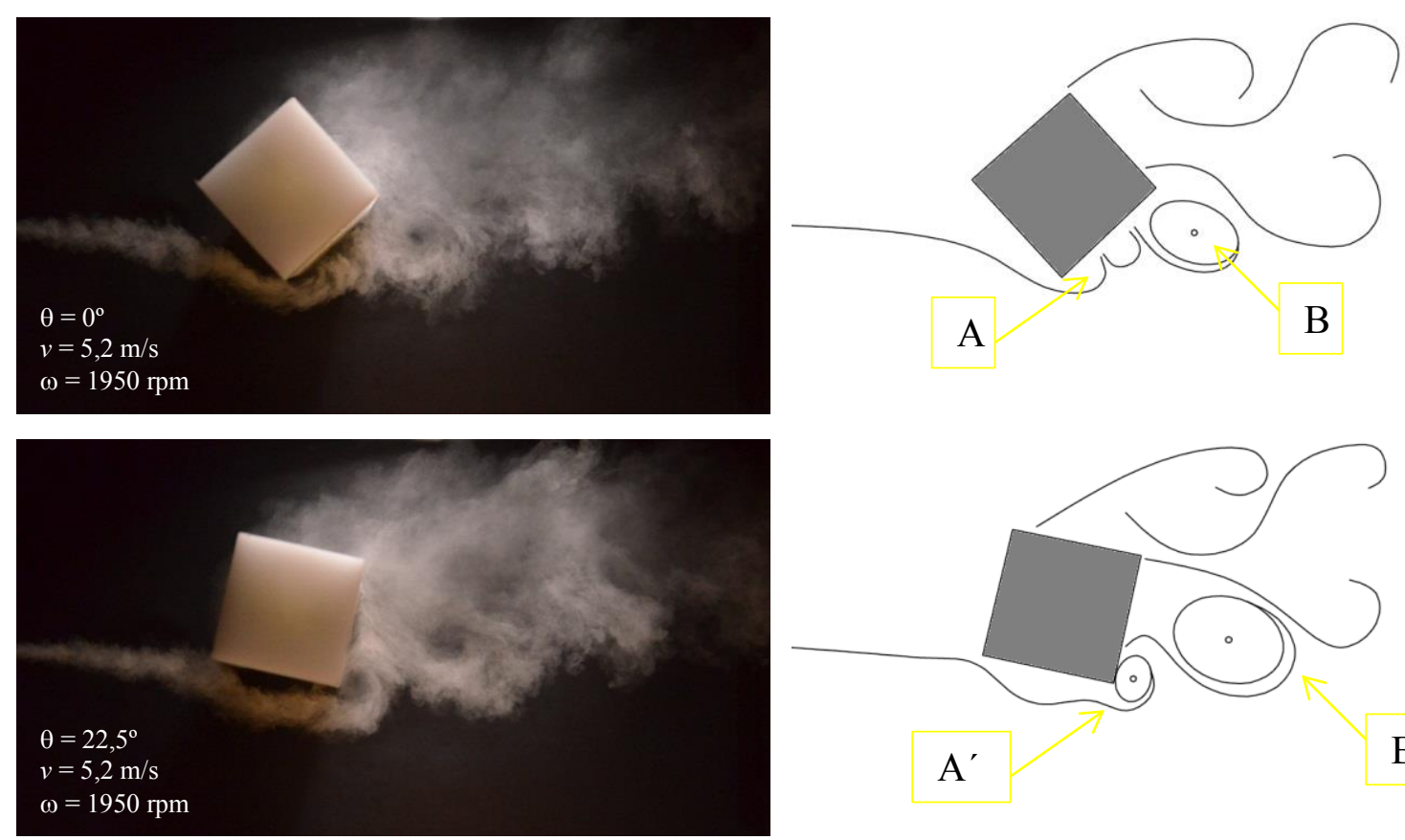

$v=5,2 \mathrm{~m} / \mathrm{s}$

$\omega=1950 \mathrm{rpm}$

$$
A^{\prime}
$$
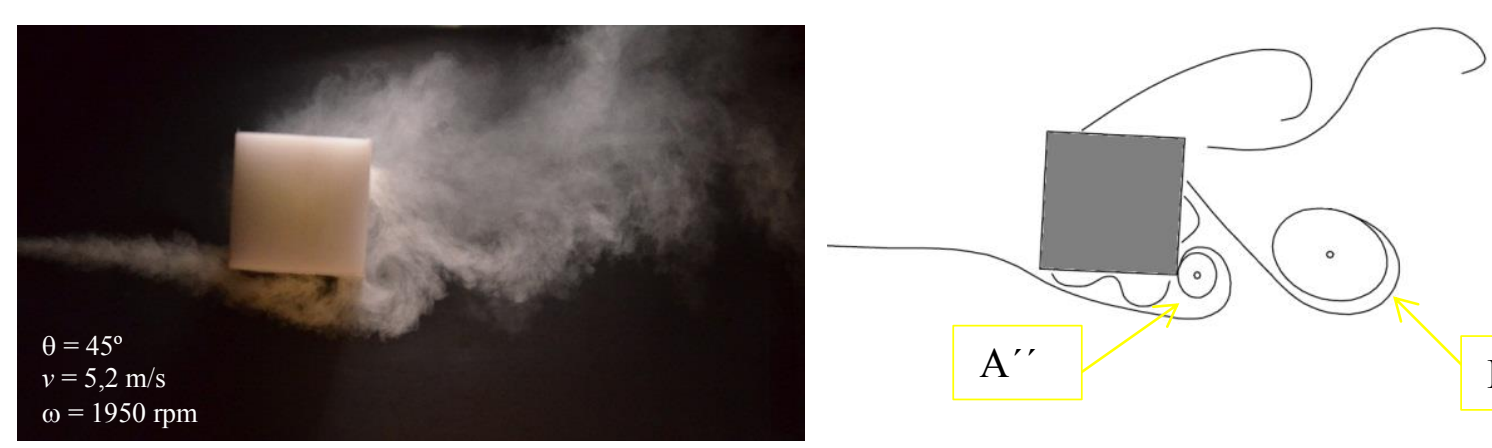

$$
A^{\prime \prime}
$$

$\mathrm{B}^{\prime \prime}$
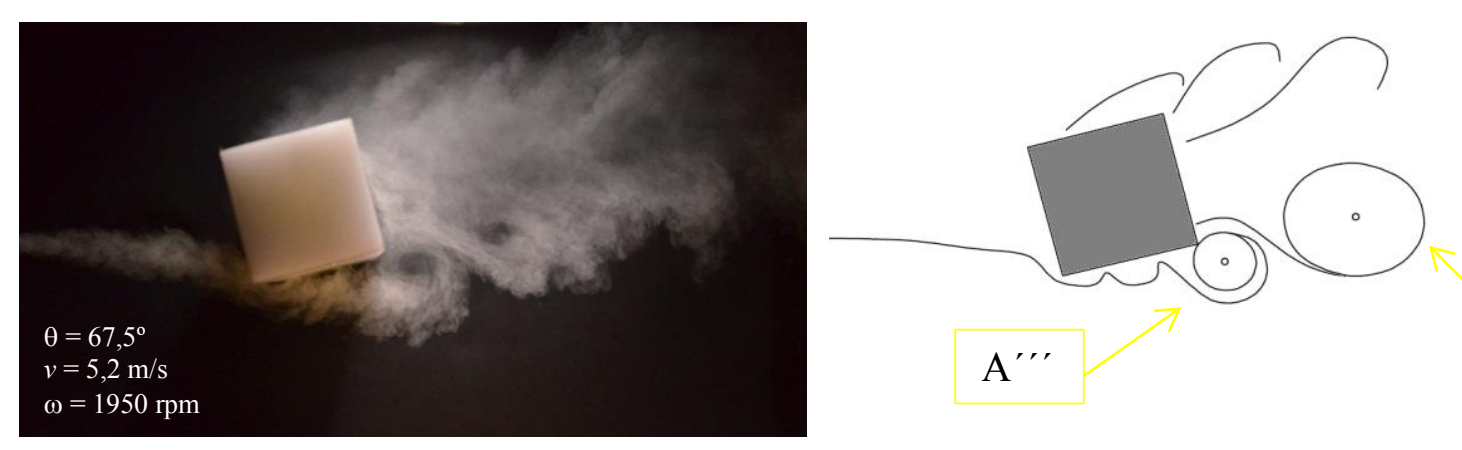

$\mathrm{A}^{\prime \prime \prime}$

$\mathrm{B}^{\prime \prime \prime}$

Figura 3.10 Configuración de flujo entorno al cuerpo en cada posición $\theta$. 
En la figura 3.11 se observa el comportamiento del flujo para el cuadrado de aristas redondeadas.
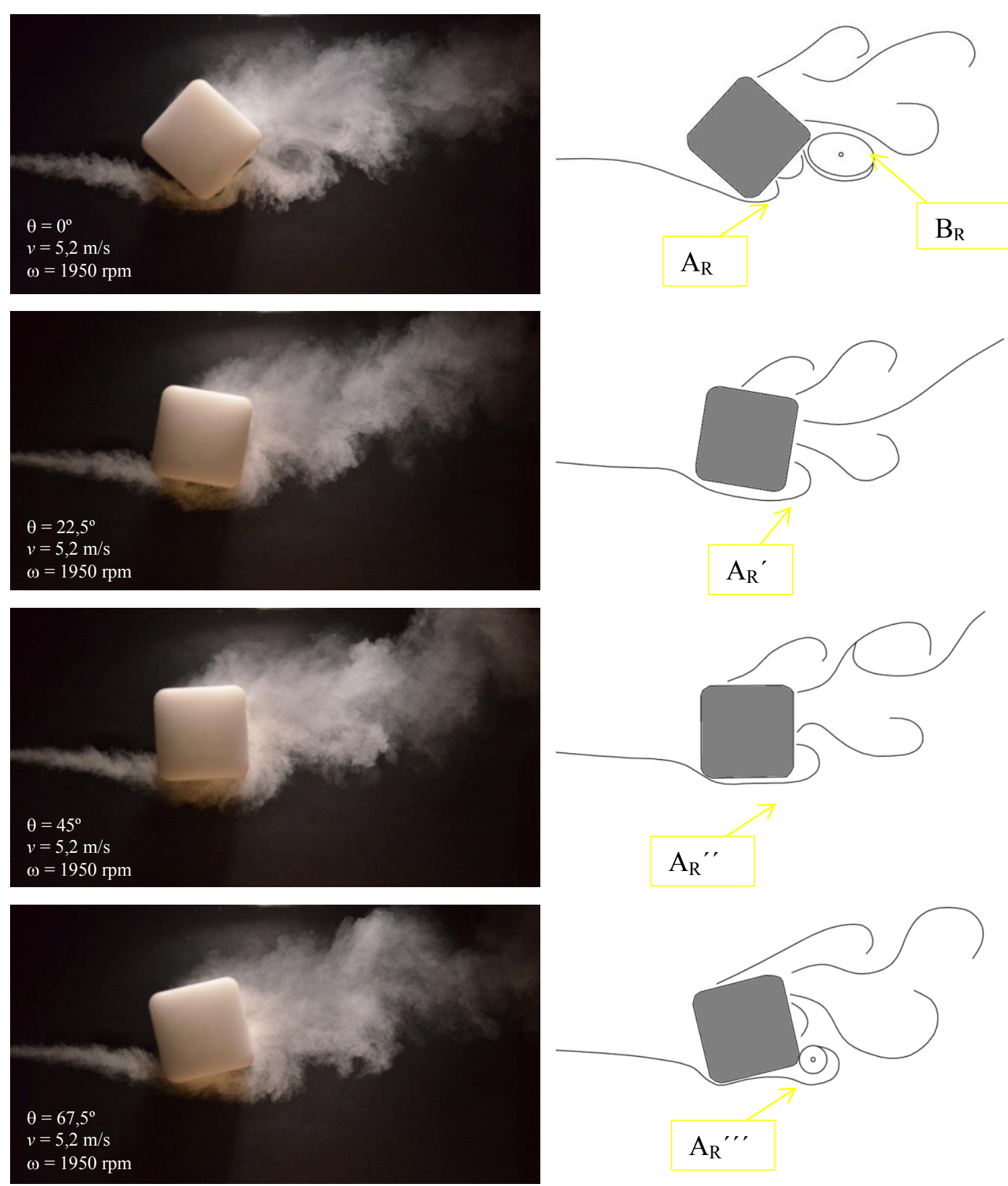

Figura 3.11 Configuración de flujo entorno al cuerpo en cada posición $\theta$. 
Para el cuadrado y el cuadrado de aristas redondeadas se observa que:

$\theta=0^{\circ}$ : comienzo de generación de los torbellinos $\mathrm{A}$ y $\mathrm{A}_{\mathrm{R}}$.

Los torbellinos $\mathrm{B}$ y $\mathrm{B}_{\mathrm{R}}$ producto del ciclo anterior son de tamaño similar en ambos casos.

En la cara inferior el flujo se encuentra más adherido en el cuadrado con bordes redondeados.

$\theta=22,5^{\circ}$ : el torbellino $A$ (en $A^{\prime}$ ) ya se ha desarrollado en tanto que el $A_{R}$ (en $A_{R}{ }^{\prime}$ ) continúa formándose.

El torbellino B (en $B^{\prime}$ ) continúa desplazándose corriente abajo. En tanto que el torbellino $B_{R}$ se ha disuelto en la estela turbulenta.

Parte del flujo que ingresa por debajo queda remansado detrás del cuerpo y cuando este rota llega hasta la cara superior para luego desprenderse.

$\theta=45^{\circ}$ : el torbellino $\mathrm{A}$ (en $\mathrm{A}^{\prime \prime}$ ) ya desarrollado queda pegado a la arista del cuerpo. El torbellino $\mathrm{A}_{\mathrm{R}}$ (en $\mathrm{A}_{\mathrm{R}}{ }^{\prime \prime}$ ) continúa formándose, no se encuentra definido.

El flujo en la cara superior continúa desprendiéndose.

$\theta=67,5^{\circ}:$ se observa el final del ciclo. El torbellino $\mathrm{A}$ (en $\mathrm{A}^{\prime \prime}$ ) ya desarrollado completamente está por desprenderse para ser el nuevo torbellino B (para $\theta=90)$.

El torbellino $A_{R}\left(e n A_{R}{ }^{\prime \prime \prime}\right)$ ya se ha formado completamente y está bien definido.

En ambos casos continúa el flujo en la cara superior producto de la rotación del cuerpo. 


\section{R2 - Relación $u / v=0,47(\omega=1950 \mathrm{rpm}, v=7,7 \mathrm{~m} / \mathrm{s})$}

En la figura 3.12 se observa el comportamiento del flujo para el cuadrado.
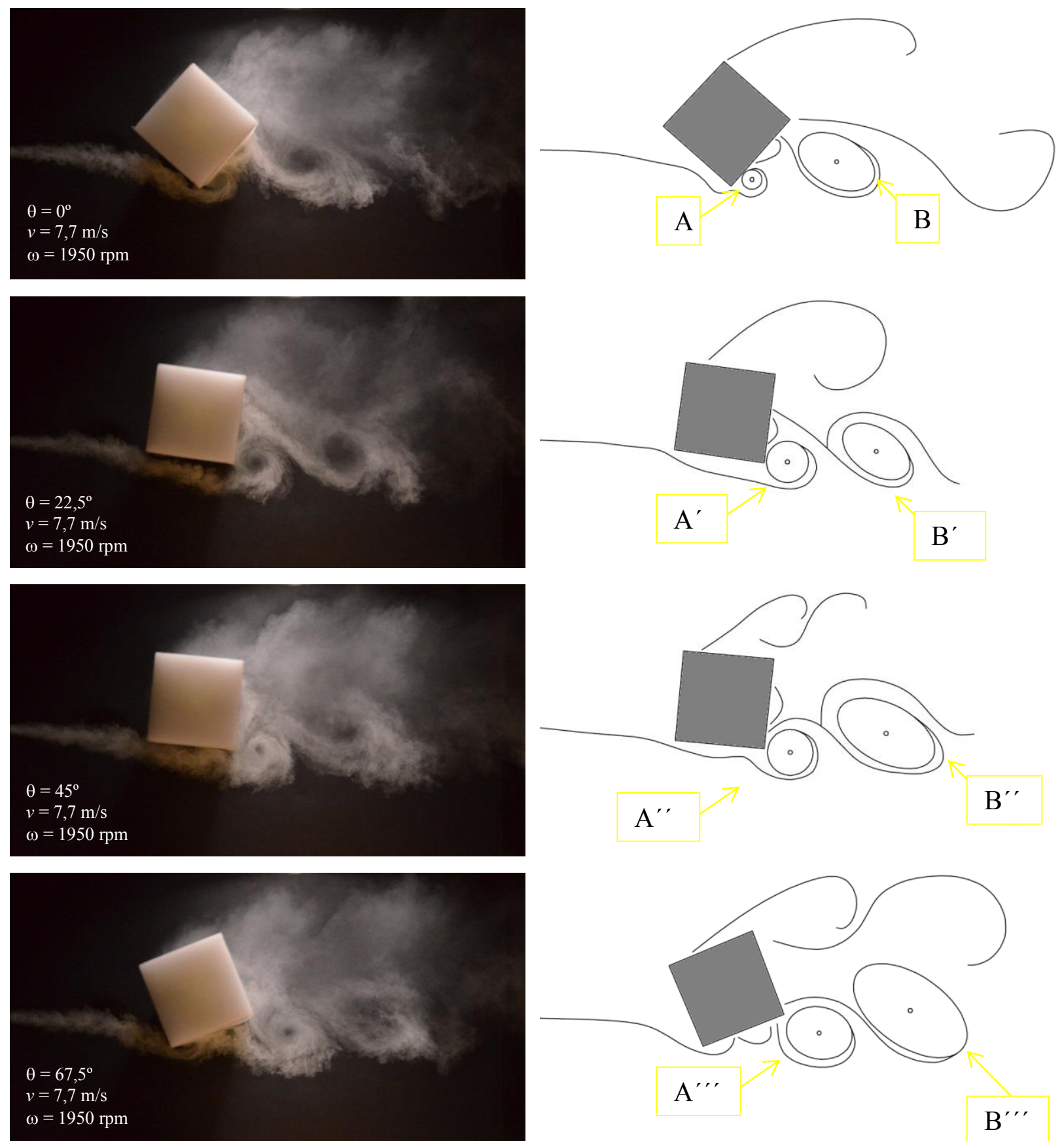

Figura 3.12 Configuración de flujo entorno al cuerpo en cada posición $\theta$. 
En la figura 3.13 se observa el comportamiento del flujo para el cuadrado con aristas redondeadas.
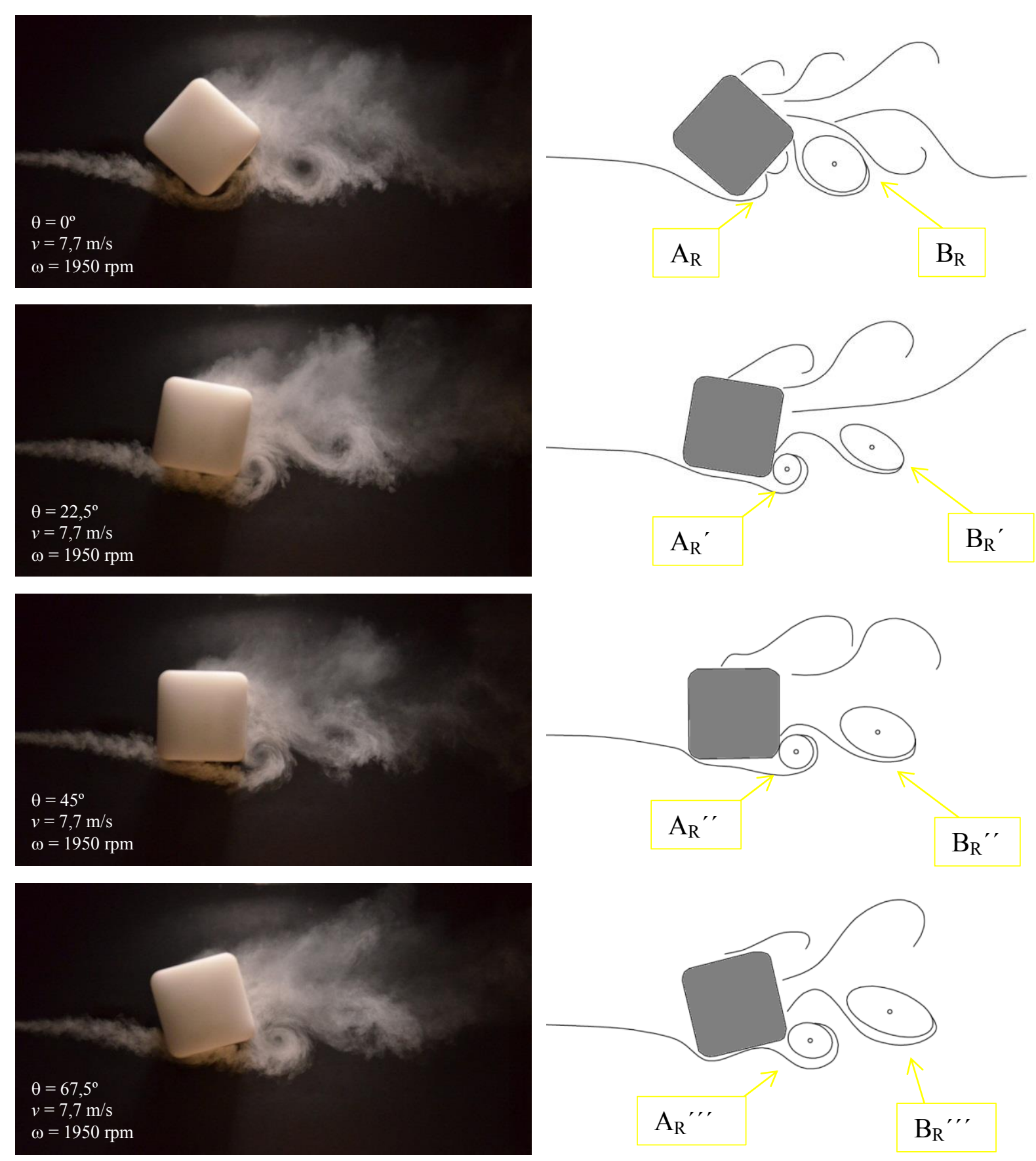

Figura 3.13 Configuración de flujo entorno al cuerpo en cada posición $\theta$. 
$\theta=0^{\circ}$ : comienza la generación del torbellino $\mathrm{A}$, en tanto que en cuadrado con aristas redondeadas comienza a desprenderse el flujo en $A_{R}$.

El torbellino B producto del ciclo anterior es de mayor tamaño que el $B_{R}$.

$\theta=22,5^{\circ}:$ los torbellinos $\mathrm{A}\left(\mathrm{en}^{\prime}\right)$ y $\mathrm{A}_{\mathrm{R}}\left(\mathrm{en}_{\mathrm{R}}{ }^{\prime}\right)$ ya se han desarrollado completamente. Los torbellinos $\mathrm{A}$ y $\mathrm{B}$ son de mayor tamaño que el $\mathrm{A}_{\mathrm{R}} \mathrm{y}$ el $\mathrm{B}_{\mathrm{R}}$.

El torbellino $B\left(\right.$ en $\left.B^{\prime}\right)$ se encuentra más alejado del cuerpo que el $B_{R}\left(\right.$ en $\left.B_{R}{ }^{\prime}\right)$.

En la cara inferior el flujo se encuentra más adherido en el cuadrado con bordes redondeados.

Parte del flujo que ingresa por debajo queda remansado detrás del cuerpo y cuando este rota llega hasta la cara superior para luego desprenderse.

$\theta=45^{\circ}$ : todos los torbellinos continúan creciendo en tamaño. Los torbellinos $\mathrm{B}$ y $\mathrm{B}_{\mathrm{R}}$ continúan desplazándose corriente abajo.

$\theta=67,5^{\circ}:$ se observa el final del ciclo. El torbellino A (en $\left.\mathrm{A}^{\prime \prime \prime}\right)$ comienza a desprenderse del cuerpo, en tanto que $A_{R}$ (en $A_{R}{ }^{\prime \prime \prime}$ ) continúa adherido al mismo. Todos los torbellinos continúan creciendo en tamaño. Los torbellinos $\mathrm{B}$ y $\mathrm{B}_{\mathrm{R}}$ continúan desplazándose corriente abajo.

En la arista inferior izquierda comienza un desprendimiento de flujo en el cuadrado. Esto no ocurre en el otro cuerpo debido a su borde redondeado.

El flujo en la cara superior comienza a desprenderse del cuerpo para continuar con la estela corriente abajo. 


\section{R3 - Relación $u / v=0,36(\omega=1950 \mathrm{rpm}, v=10,2 \mathrm{~m} / \mathrm{s})$}

En la figura 3.14 se observa el comportamiento del flujo para el cuadrado.
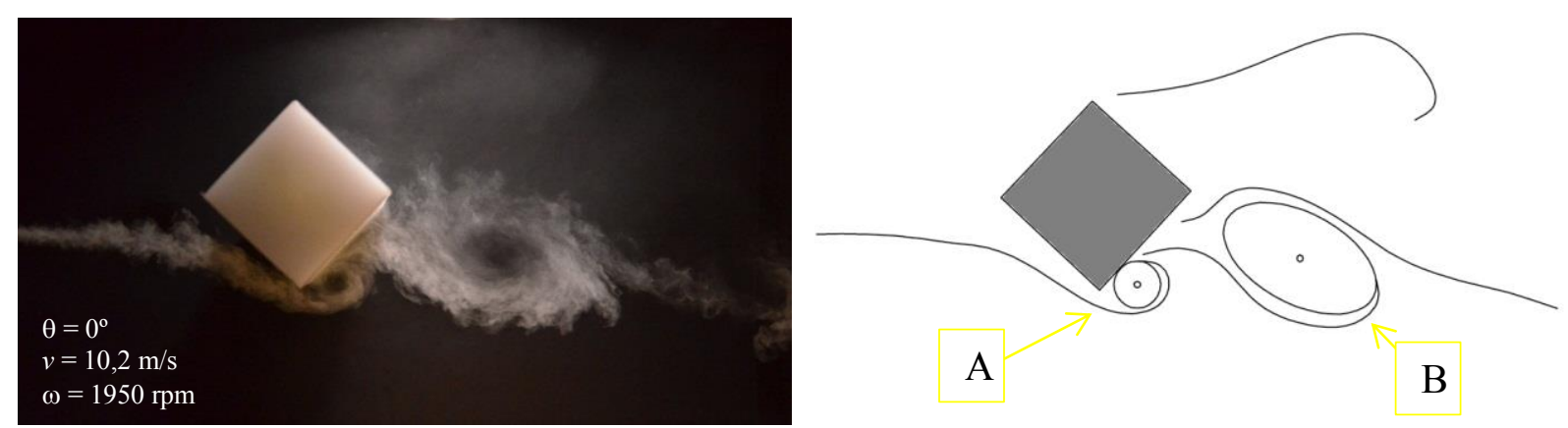

A

B
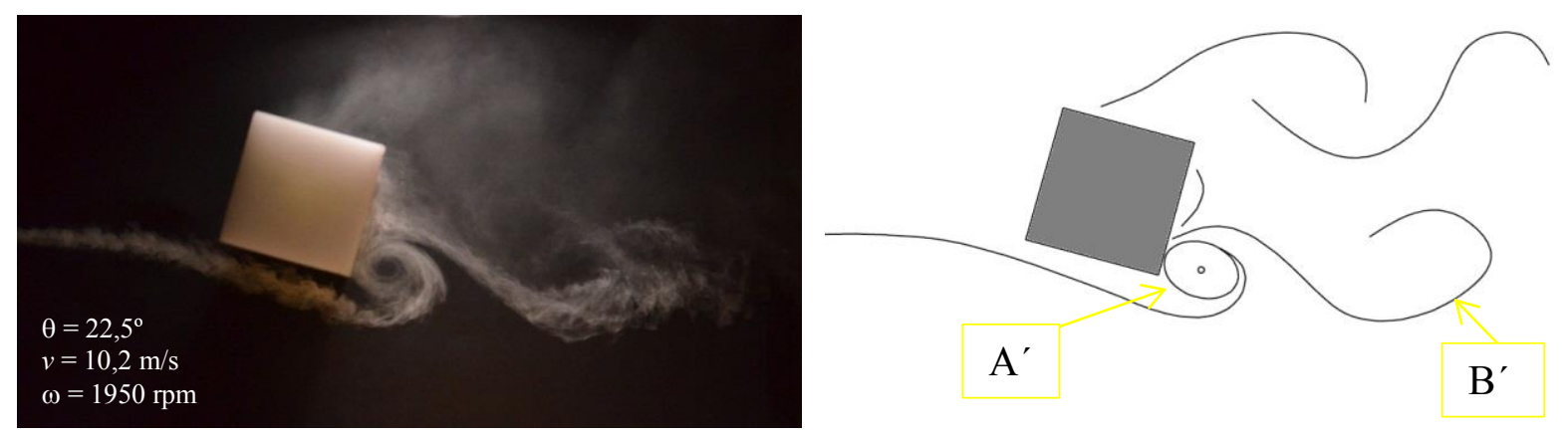

$v=10,2 \mathrm{~m} / \mathrm{s}$

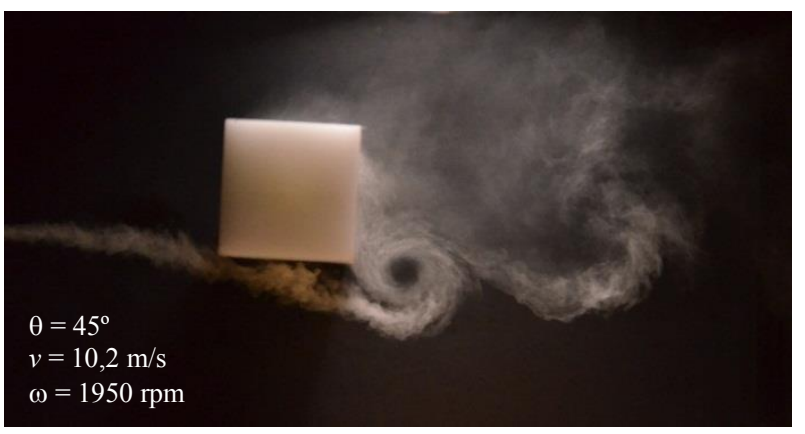

$\mathrm{A}^{\prime}$

$\mathrm{B}^{\prime}$

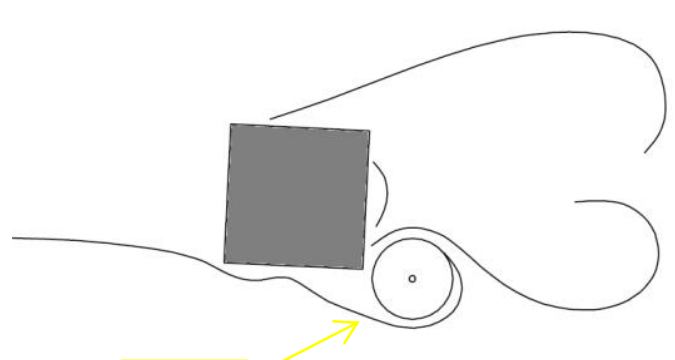

$\mathrm{A}^{\prime \prime}$
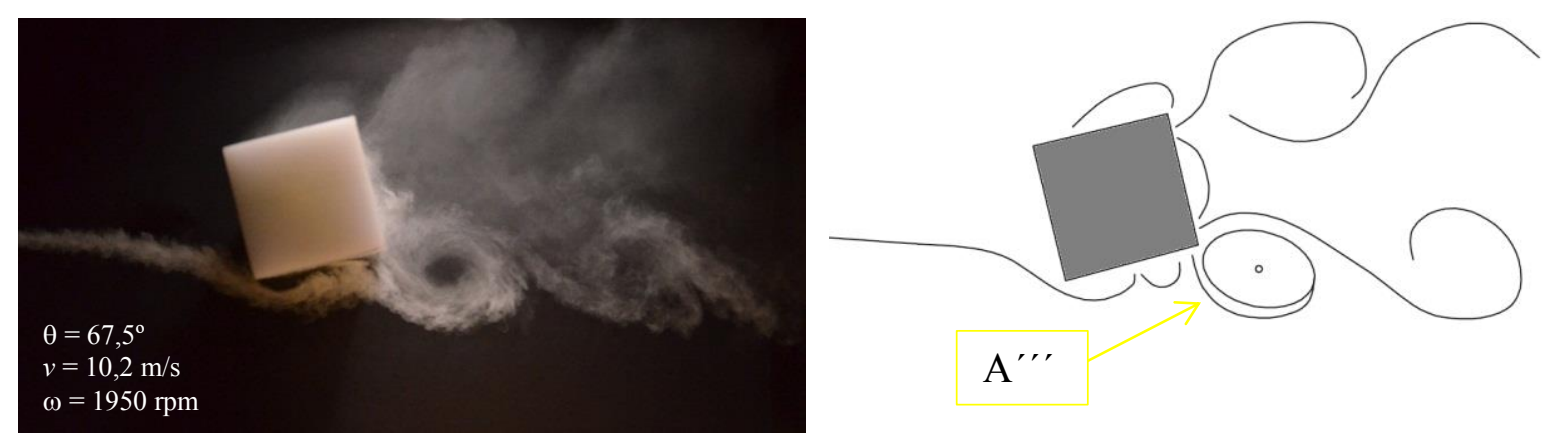

Figura 3.14 Configuración de flujo entorno al cuerpo en cada posición $\theta$. 
En la figura 3.15 se observa el comportamiento del flujo para el cuadrado con aristas redondeadas.
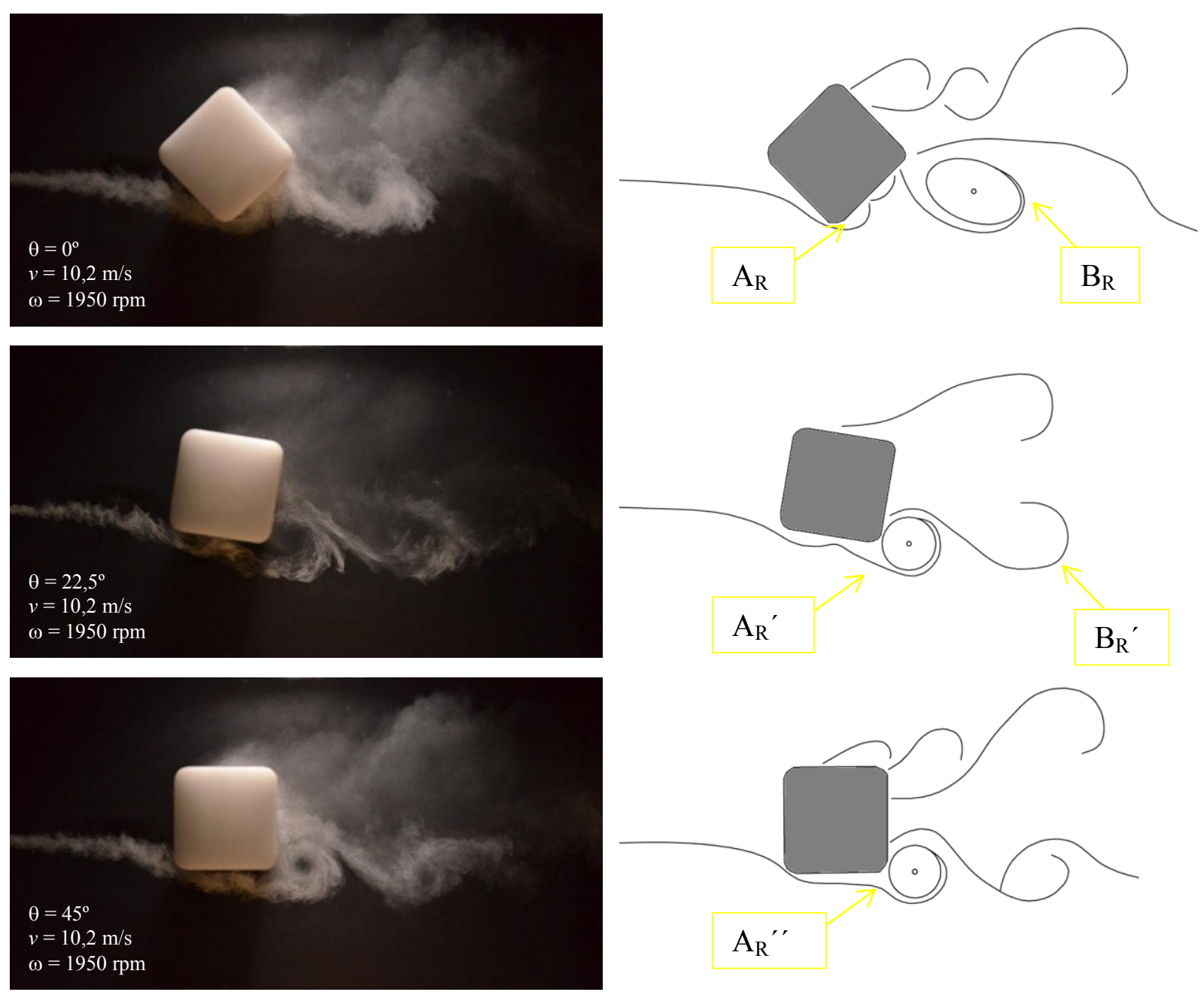

$v=10,2 \mathrm{~m} / \mathrm{s}$

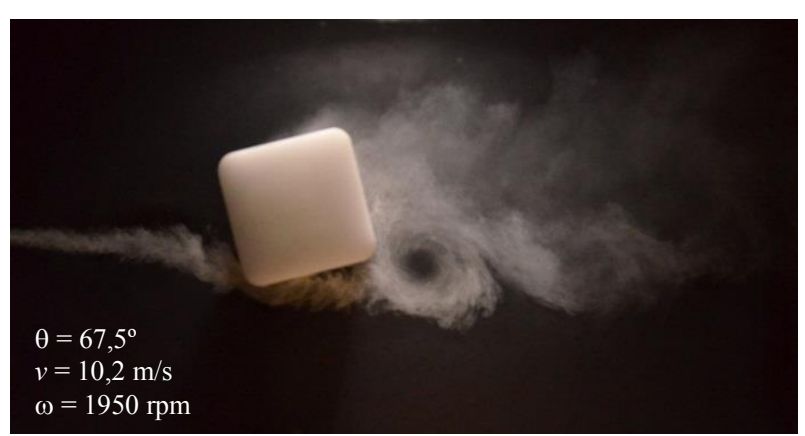

$$
\mathrm{A}_{\mathrm{R}}{ }^{\prime \prime}
$$

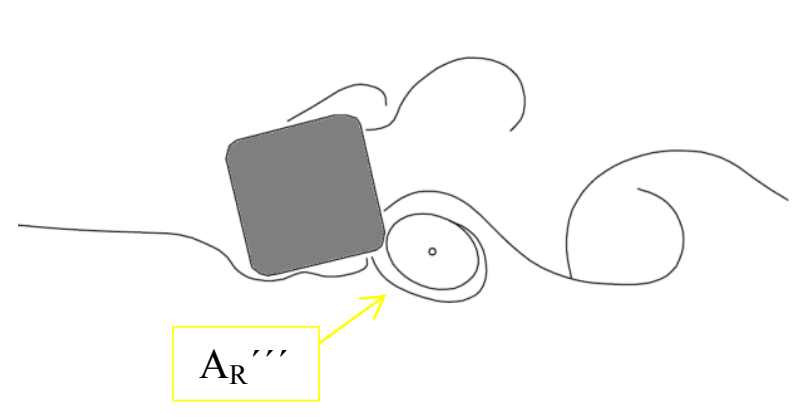

Figura 3.15 Configuración de flujo entorno al cuerpo en cada posición $\theta$. 
$\theta=0^{\circ}$ : el torbellino A se produce por efecto de la arista afilada. El torbellino $A_{R}$ está en proceso de formación.

El torbellino $\mathrm{B}$, producto del ciclo anterior, es de mayor tamaño que el $\mathrm{B}_{\mathrm{R}}$ y se encuentra más separado del cuerpo.

$\theta=22,5^{\circ}:$ los torbellinos $\mathrm{A}\left(\mathrm{en} \mathrm{A}^{\prime}\right)$ y $\mathrm{A}_{\mathrm{R}}\left(\mathrm{en} \mathrm{A}_{\mathrm{R}}{ }^{\prime}\right)$ ya se han desarrollado completamente y se ubican sobre la cara trasera.

Los torbellinos $\mathrm{B}$ y $\mathrm{B}_{\mathrm{R}}$ del ciclo anterior están en proceso de desintegración. Así mismo el $\mathrm{B}$ (en $\left.B^{\prime}\right)$ es de mayor tamaño y se encuentra más alejado que el $B_{R}\left(\right.$ en $\left.B_{R}{ }^{\prime}\right)$.

Los torbellinos $\mathrm{A}$ y $\mathrm{B}$ son de mayor tamaño que el $\mathrm{A}_{\mathrm{R}}$ y el $\mathrm{B}_{\mathrm{R}}$.

En la cara inferior el flujo se encuentra más adherido en el cuadrado con bordes redondeados.

Parte del flujo que ingresa por debajo queda remansado detrás del cuerpo y cuando este rota llega hasta la cara superior para luego desprenderse.

$\theta=45^{\circ}$ : todos los torbellinos continúan creciendo en tamaño. Los torbellinos $\mathrm{B}$ y $\mathrm{B}_{\mathrm{R}}$ continúan desplazándose corriente abajo y comienzan a deshacerse en la estela.

$\theta=67,5^{\circ}$ : se observa el final del ciclo. El torbellino A (en $\mathrm{A}^{\prime \prime \prime}$ ) comienza a desprenderse del cuerpo, en tanto que $A_{R}$ (en $\left.A_{R}{ }^{\prime \prime \prime}\right)$ continúa adherido al mismo. Todos los torbellinos continúan creciendo en tamaño. Los torbellinos $\mathrm{B}$ y $\mathrm{B}_{\mathrm{R}}$ están prácticamente mezclados en la estela y ya no están definidos.

En la arista inferior izquierda comienza un desprendimiento de flujo en el cuadrado. Esto no ocurre en el otro cuerpo debido a su borde redondeado.

El flujo en la cara superior comienza a desprenderse del cuerpo para continuar con la estela corriente abajo. 


\section{R4 - Relación $u / v=0,6(\omega=2520 \mathrm{rpm}, v=7,7 \mathrm{~m} / \mathrm{s})$}

En la figura 3.16 se observa el comportamiento del flujo para el cuerpo circular con aletas.
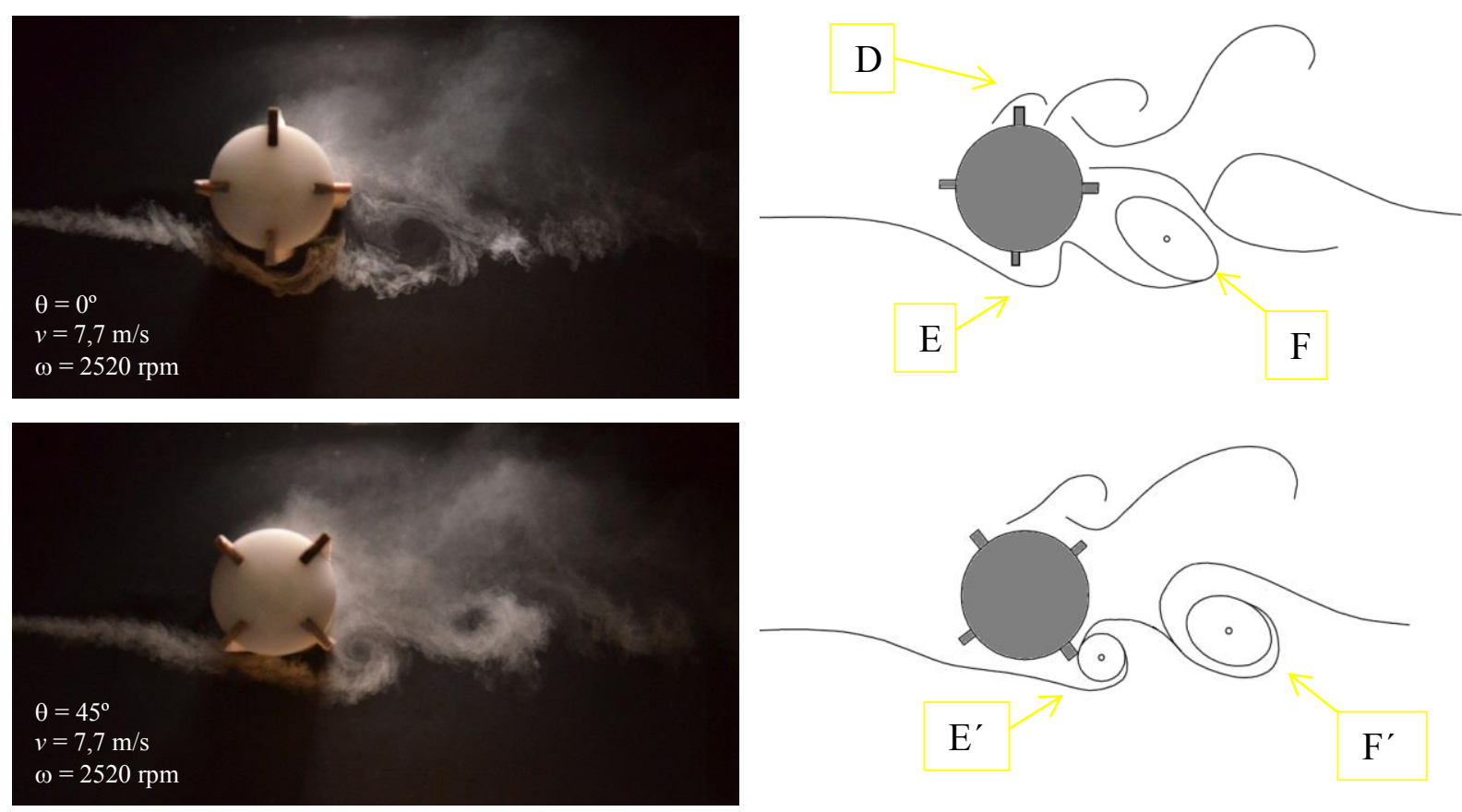

Figura 3.16 Configuración de flujo entorno al cuerpo en cada posición $\theta$.

$\theta=0^{\circ}$ : comienza la formación del torbellino $\mathrm{E}$ detrás de la aleta. El torbellino $\mathrm{F}$ es el generado en el ciclo anterior.

Parte del flujo que ingresa por debajo queda remansado detrás del cuerpo entre las aletas y cuando este rota llega hasta la parte superior para luego desprenderse.

$\theta=45^{\circ}$ : final del ciclo. El torbellino $\mathrm{E}$ (en E') está completamente formado detrás de la aleta. El torbellino $\mathrm{F}$ (en $\mathrm{F}^{\prime}$ ) se aleja con la estela. 


\subsubsection{Conclusiones familia II}

En el caso del cuerpo circular liso con aletas, como las mismas son de una altura pequeña (menor que el 10\% del lado) y poseen bordes afilados, se puede decir que se comporta como un cuadrado de aristas afiladas (figuras 3.8 y 3.9 ).

A medida que aumenta la relación de velocidades $u / v$, los patrones de flujo de los tres cuerpos tienden a ser similares (ver patrones en figuras 3.10, 3.11 y 3.16). El tamaño del desprendimiento producto de las aristas es menor. Dicho fenómeno explica la tendencia de los tres cuerpos a converger en un valor de $C_{L}$ para altos valores de $u / v$ (figura 3.8).

En viceversa, a medida que disminuye la relación de velocidades $u / v$, más grandes son los torbellinos generados y más separación tienen estos, respecto a los cuerpos, lo que explica las diferencias en los valores de $C_{D}$ para los cuerpos (figuras 3.9).

Puede observarse un desfasaje en la posición de los cuerpos para la formación de torbellinos en la que el cuadrado y el circular liso con aletas generan el torbellino en $\theta=67,5^{\circ}$ (figuras 3.10, posición $\mathrm{A}^{\prime \prime \prime}$ ) y $\theta=45^{\circ}$ (figuras 3.16, posición $\mathrm{E}^{\prime}$ ) respectivamente (mismas posiciones considerando el circular con aletas como un cuadrado) y el cuadrado con bordes redondeados en $\theta=0^{\circ}$ (figuras 3.13, posición $A_{R}{ }^{\prime}$ ).

Así mismo, la diferencia en los valores de $C_{D}$ del circular con aletas respecto a los otros puede explicarse debido a la turbulencia generada entre aleta y aleta, y por ende, la estela turbulenta. A medida que aumenta $v$ más turbulenta y ancha se vuelve la estela, aumentando el $C_{D}$. El $C_{D}$ es como el del cuadrado para relaciones de $u / v<0,8$, a partir de valores mayores de $u / v$ comienza la diferencia.

En los tres cuerpos los valores de $C_{D}$ inicialmente decaen para aumentar a partir de $u / v=0,7$. 


\subsubsection{Familia III}

La misma está conformada por el triángulo y el triángulo con aristas redondeadas. En las figuras 3.17 y 3.18 se observa la variación de los coeficientes aerodinámicos $C_{L}$ y $C_{D}$ de los mencionados cuerpos con la relación de velocidades $u / v$. Los puntos $\mathrm{R}$ señalados en las mismas corresponden a diferentes condiciones de $u / v$ para las que se tomaron fotografías.

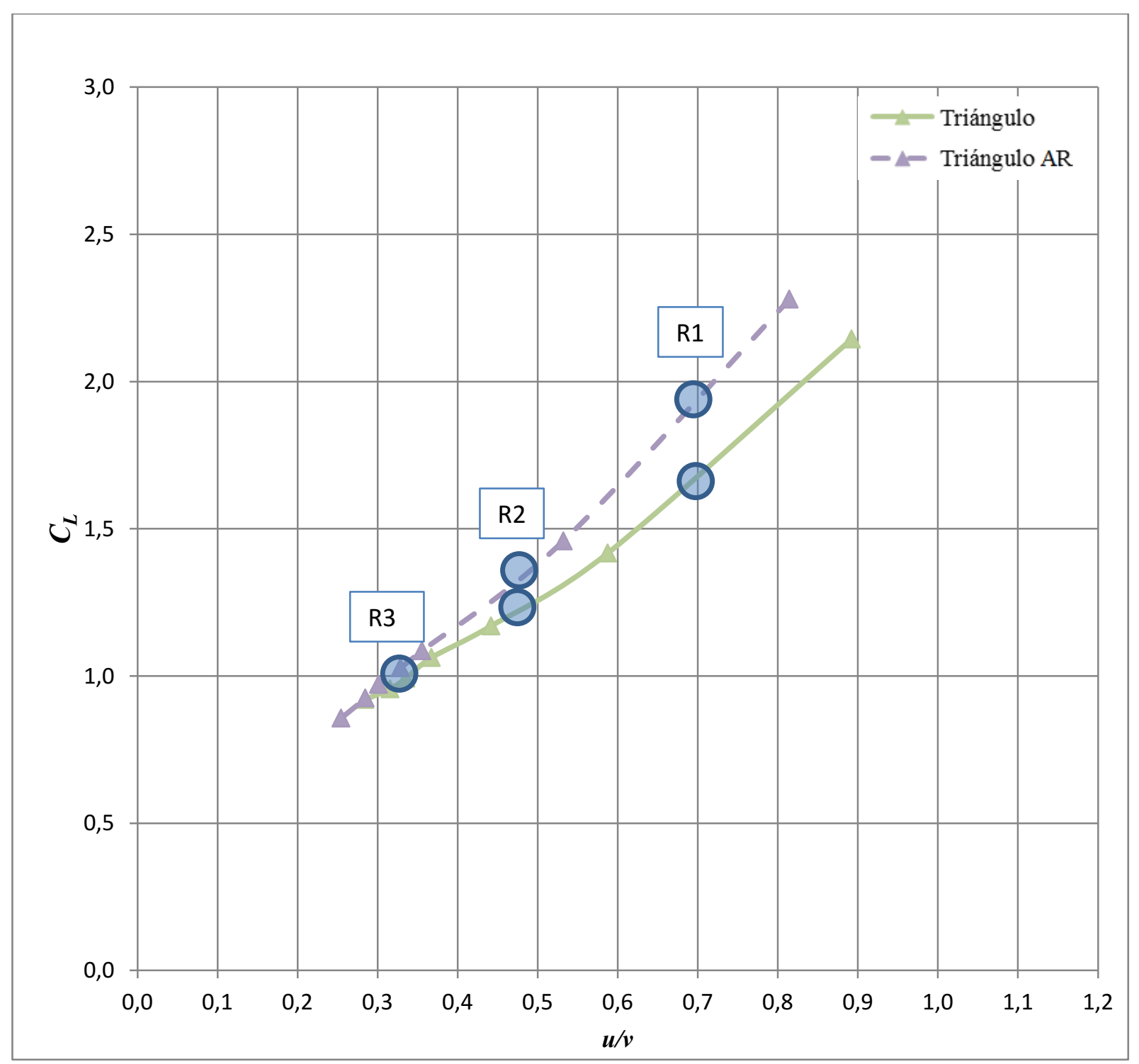

Figura 3.17 Variación del coeficiente de sustentación $C_{L}$ en función de la relación de velocidades $u / v$, y relaciones R1, R2 y R3 para el análisis fotográfico (círculos gruesos). 


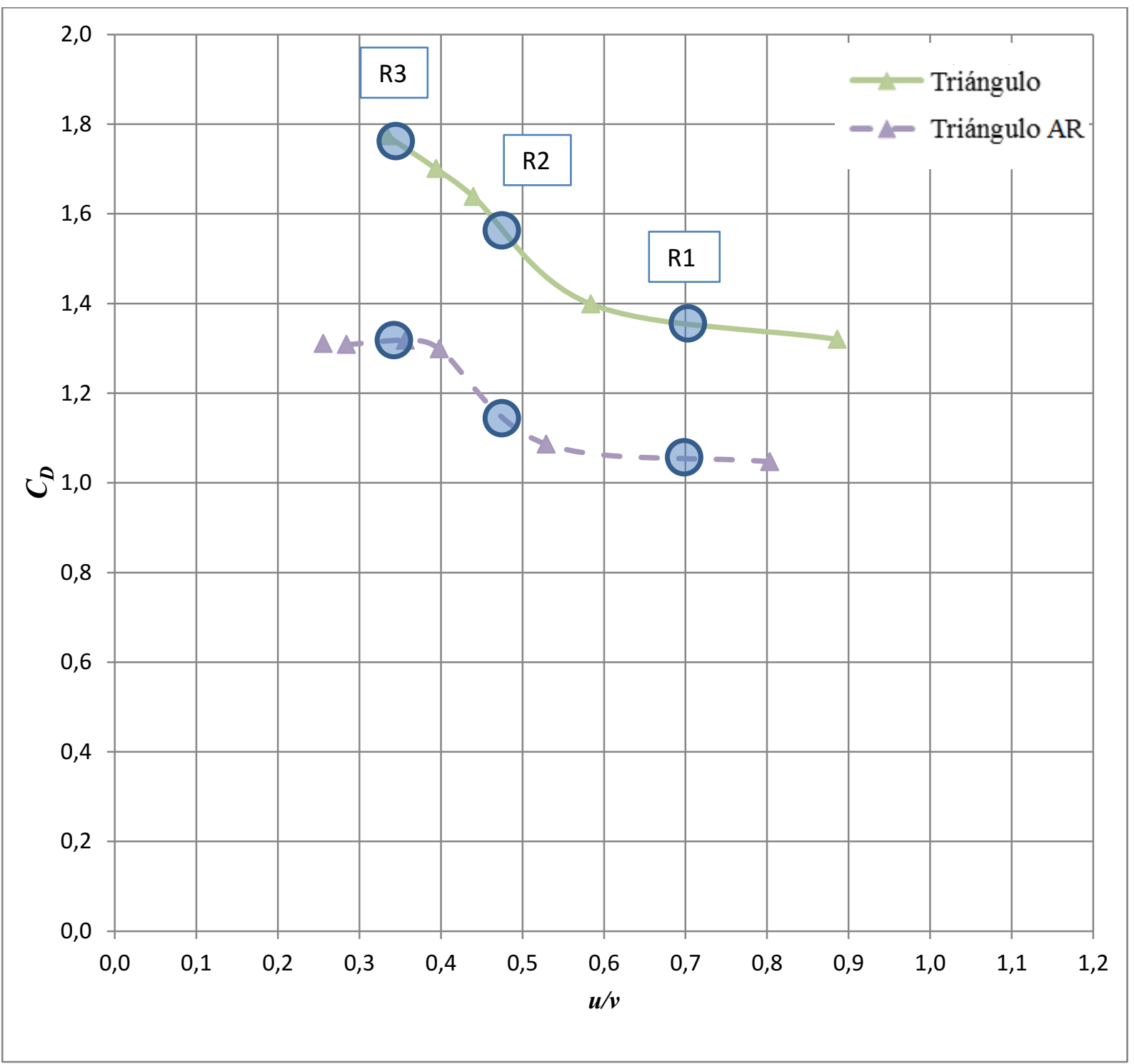

Figura 3.18 Variación del coeficiente de sustentación $C_{D}$ en función de la relación de velocidades $u / v$, y relaciones R1, R2 y R3 para el análisis fotográfico (círculos gruesos).

A continuación se realiza una descripción de los flujos correspondientes a los diversos cuerpos de la Familia III para las relaciones R1, R2 y R3. 


\section{$\mathrm{R} 1$ - Relación $u / v=0,70(\omega=1950 \mathrm{rpm}, v=5,2 \mathrm{~m} / \mathrm{s})$}

En la figura 3.19 se observa el comportamiento del flujo para el triángulo.
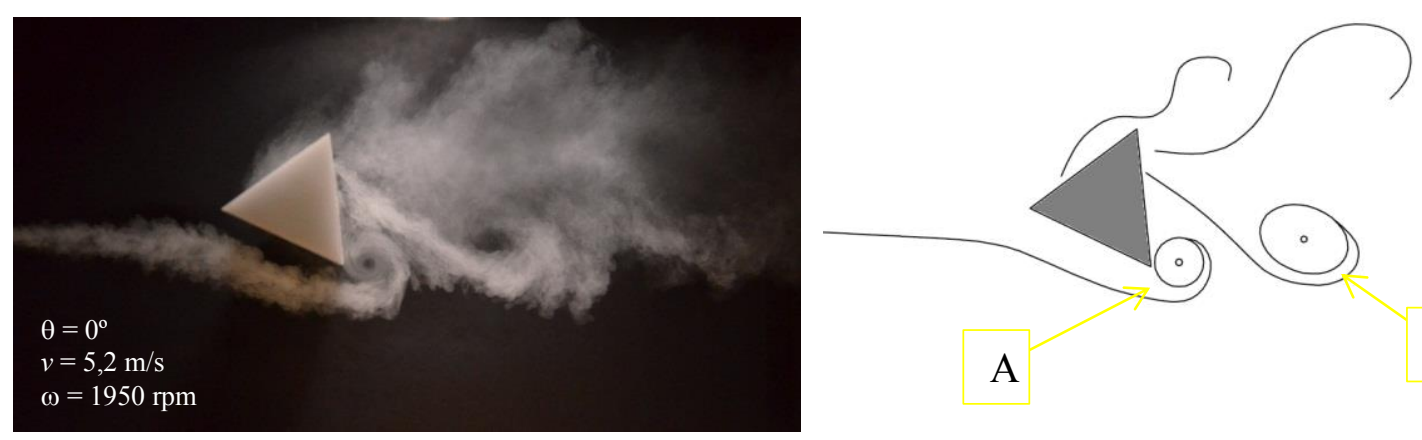

A

B
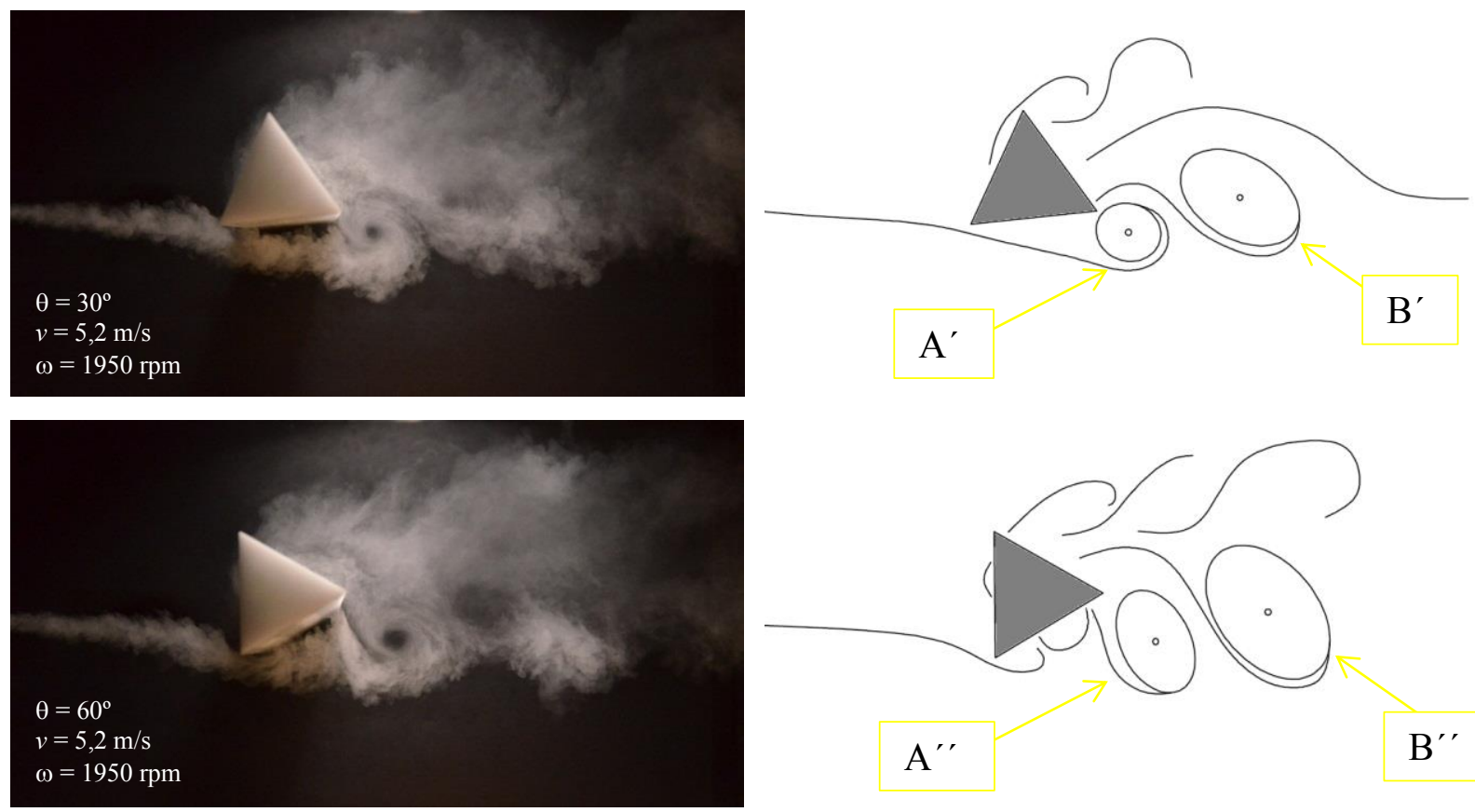

$A^{\prime \prime}$

$\mathrm{B}^{\prime \prime}$
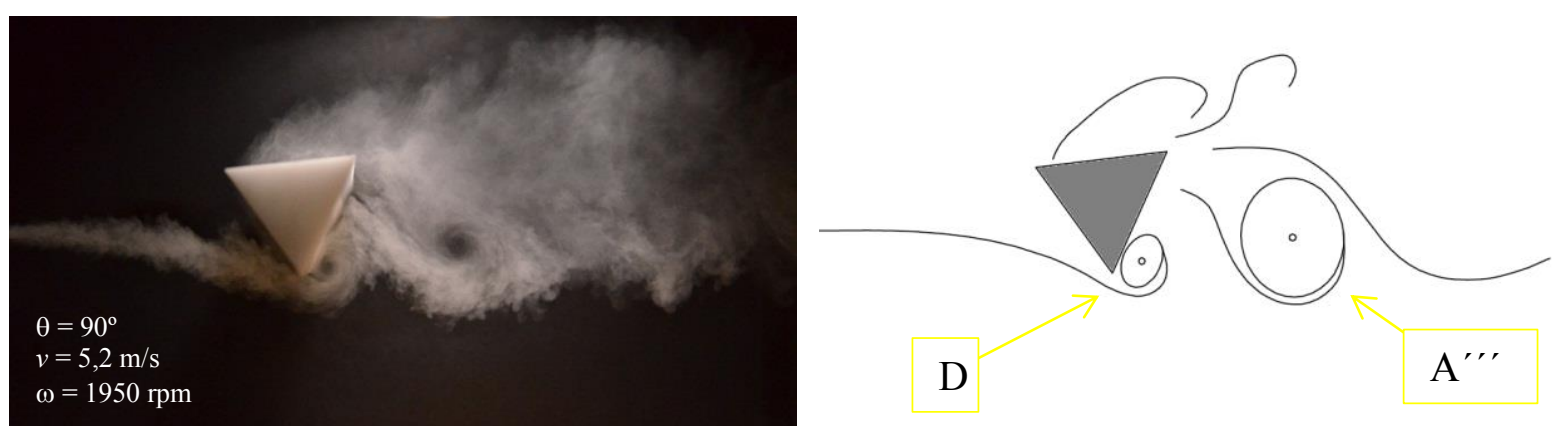

Figura 3.19 Configuración de flujo entorno al cuerpo en cada posición $\theta$. 
En la figura 3.20 se observa el comportamiento del flujo para el triángulo con aristas redondeadas.
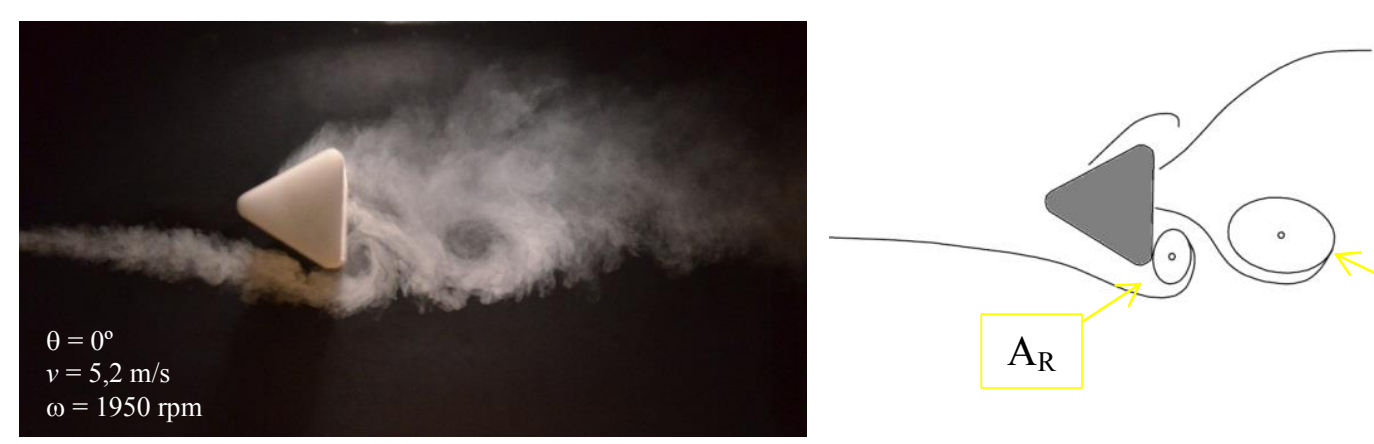

$\mathrm{A}_{\mathrm{R}}$

$\mathrm{B}_{\mathrm{R}}$
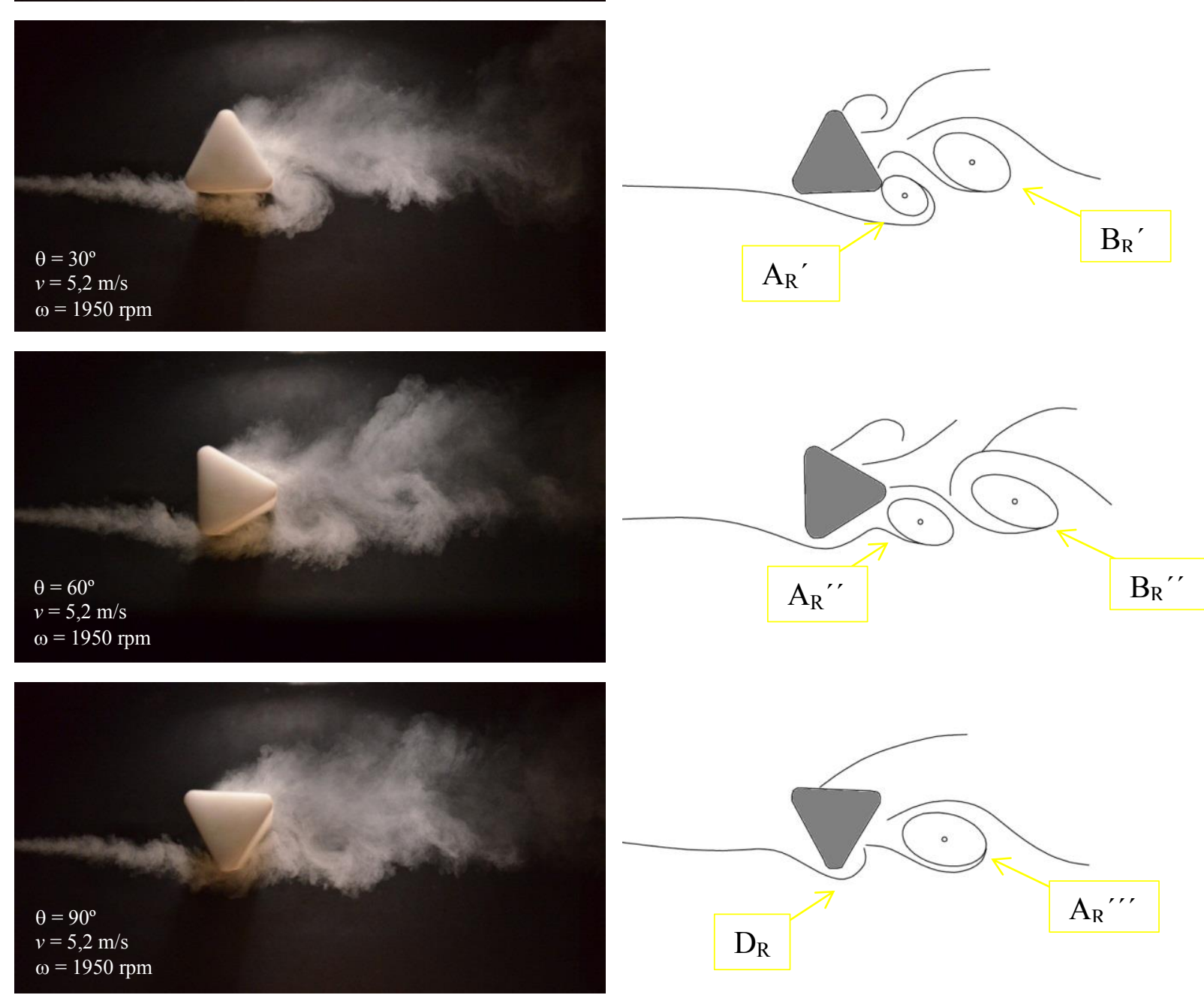

Figura 3.20 Configuración de flujo entorno al cuerpo en cada posición $\theta$. 
$\theta=0^{\circ}$ : el vértice agudo de triángulo genera un torbellino A más definido y de mayor tamaño que en caso del $A_{R}$. En tanto que el torbellino $B$ es de mayor tamaño y se encuentra más separado del cuerpo respecto al $\mathrm{B}_{\mathrm{R}}$.

En la cara inferior el flujo se encuentra más adherido en el triángulo con bordes redondeados.

$\theta=30^{\circ}:$ los torbellinos A (en $\left.A^{\prime}\right)$ y B (en B') aumentan su tamaño y comienzan a separarse del cuerpo. En el caso del triángulo, los mismos se separan más distancia que en el de bordes redondeados $\left(\mathrm{A}_{\mathrm{R}}{ }^{\prime}, \mathrm{B}_{\mathrm{R}}{ }^{\prime}\right)$, a su vez que el $\mathrm{A}$ se desplaza levemente hacia abajo.

$\theta=60^{\circ}$ : continúa el crecimiento de los torbellinos. El torbellino A (en $\mathrm{A}^{\prime \prime}$ ) continúa desplazándose hacia abajo, en tanto que el torbellino $A_{R}\left(\right.$ en $\left.A_{R}{ }^{\prime \prime}\right)$ mantiene su posición vertical.

Los torbellinos $\mathrm{B}\left(\right.$ en $\left.\mathrm{B}^{\prime \prime}\right)$ y $\mathrm{B}_{\mathrm{R}}\left(\right.$ en $\left._{\mathrm{R}^{\prime \prime}}\right)$ continúan desplazándose corriente abajo.

$\theta=90^{\circ}$ : se observa el final del ciclo. Comienzan a generarse los nuevos torbellinos A (en D) y $A_{R}\left(\right.$ en $\left.D_{R}\right)$ respectivos para cada cuerpo, en tanto que los anteriores continúan corriente abajo generando una calle de torbellinos.

En ambos casos existe flujo en la cara superior producto de la succión generada por el cuerpo en rotación. 


\section{R2 - Relación $u / v=0,47(\omega=1950 \mathrm{rpm}, v=7,7 \mathrm{~m} / \mathrm{s})$}

En la figura 3.21 se observa el comportamiento del flujo para el triángulo.
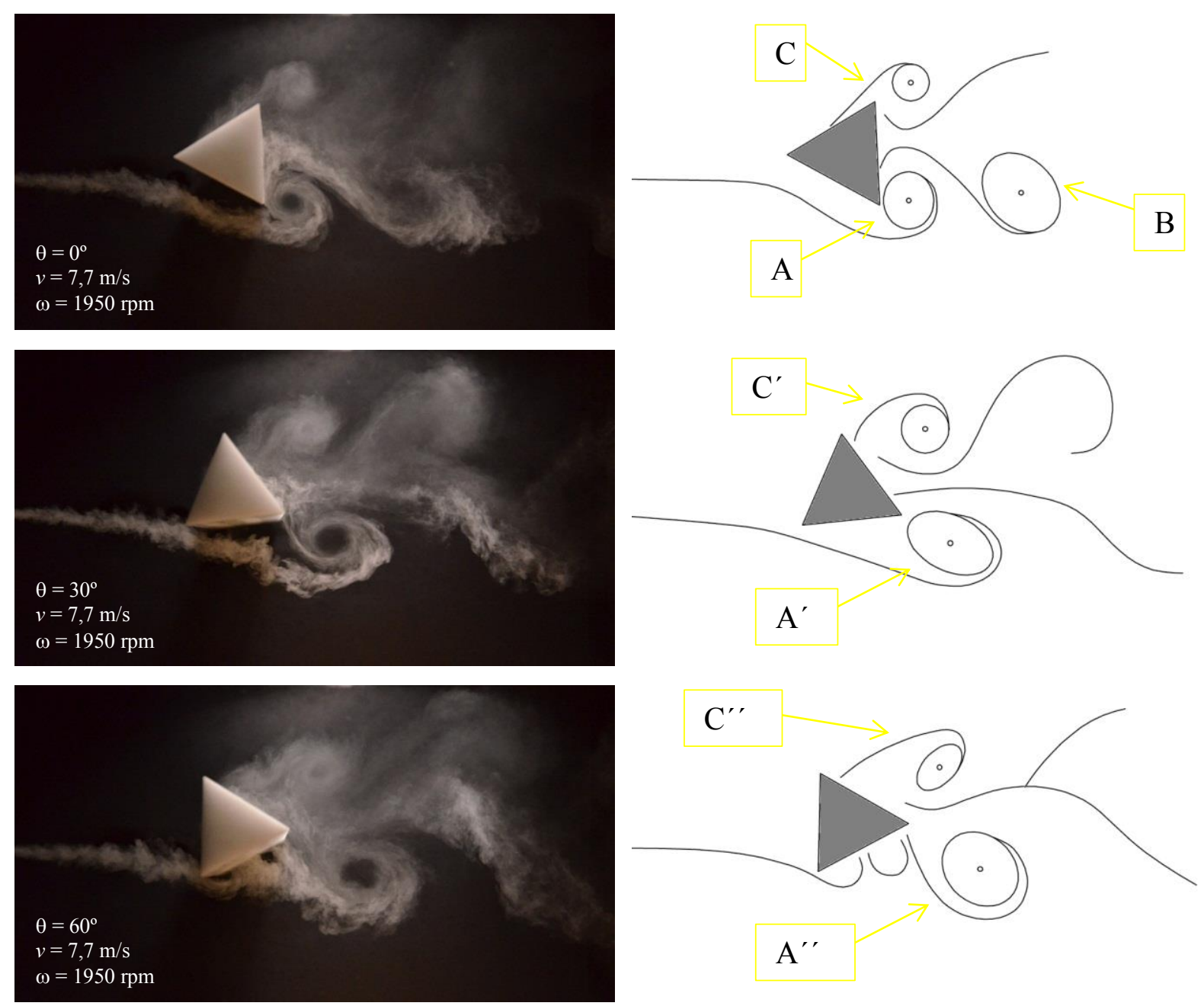

$v=7,7 \mathrm{~m} / \mathrm{s}$
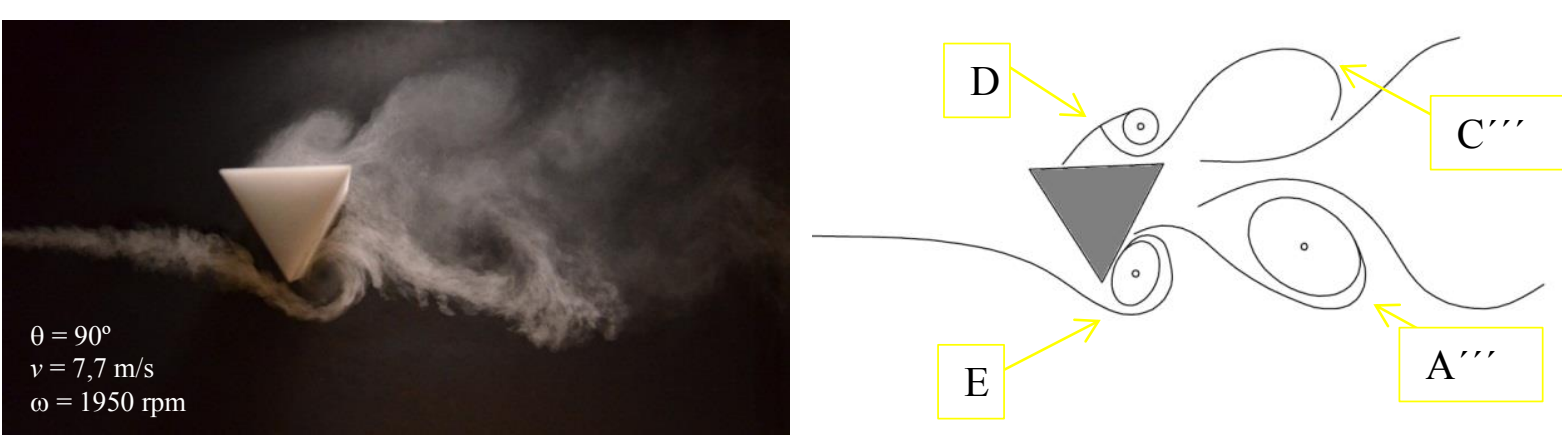

Figura 3.21 Configuración de flujo entorno al cuerpo en cada posición $\theta$. 
En la figura 3.22 se observa el comportamiento del flujo para el triángulo con aristas redondeadas.
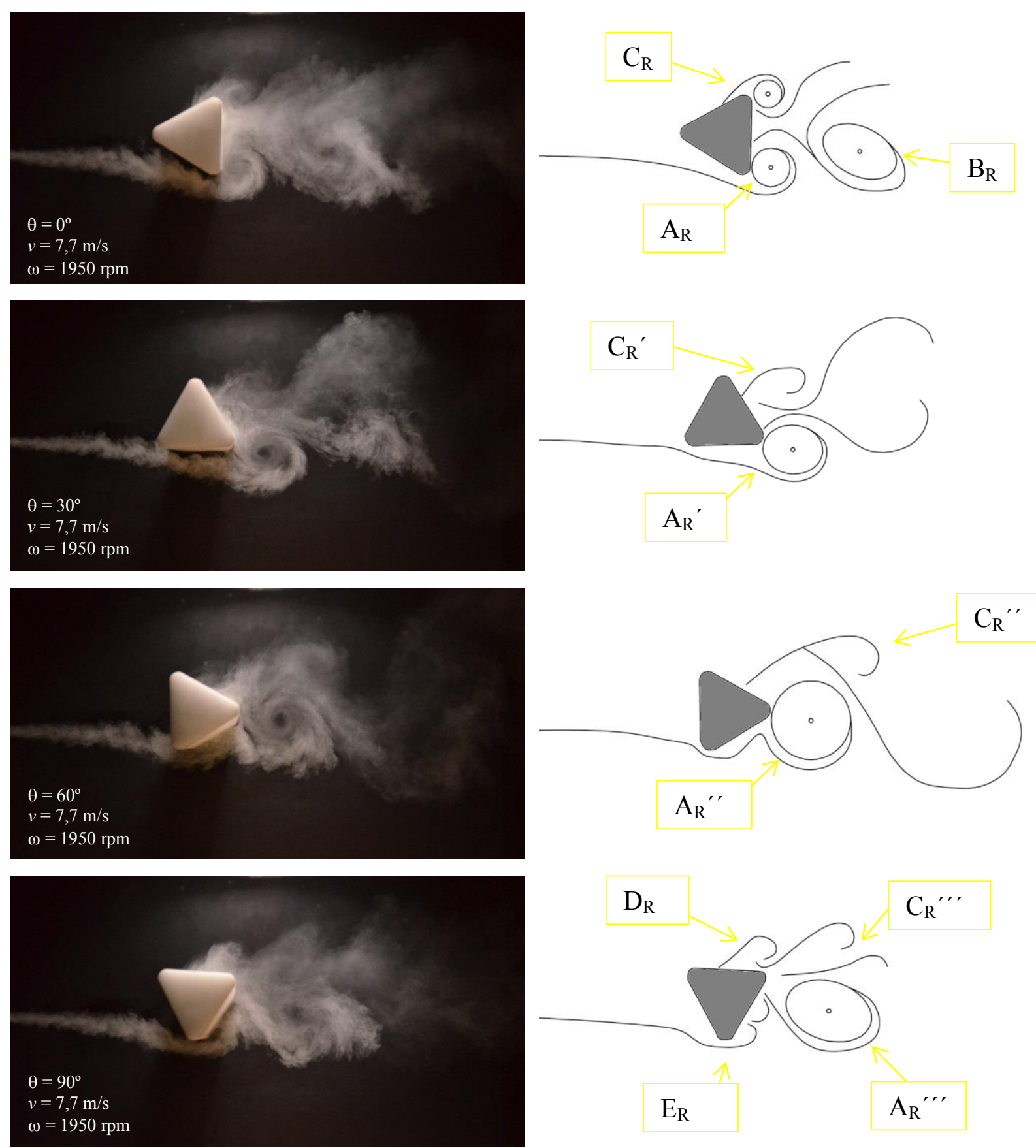

Figura 3.22 Configuración de flujo entorno al cuerpo en cada posición $\theta$. 
$\theta=0^{\circ}$ : la arista afilada del triángulo genera un torbellino A más definido y de mayor tamaño que en caso del $A_{R}$. En tanto que el torbellino $B$ es de mayor tamaño y se encuentra más separado del cuerpo respecto al $\mathrm{B}_{\mathrm{R}}$.

En ambos cuerpos se produce la generación de torbellinos con sentido horario en la capa superior. El torbellino $\mathrm{C}$ es de mayor tamaño respecto del $\mathrm{C}_{\mathrm{R}}$.

En la cara inferior el flujo se encuentra más adherido en el triángulo con bordes redondeados.

$\theta=30^{\circ}$ : los torbellinos $A\left(\right.$ en $\left.A^{\prime}\right), C\left(\right.$ en $\left.C^{\prime}\right), A_{R}\left(\right.$ en $\left.A_{R}{ }^{\prime}\right)$ y $C_{R}\left(\right.$ en $\left.C_{R}{ }^{\prime}\right)$ aumentan su tamaño y se separan del cuerpo. En el caso del triángulo, los mismos se separan más distancia que en el de bordes redondeados, a su vez que el torbellino A se desplaza hacia abajo en A'.

$\theta=60^{\circ}$ : el torbellino $A_{R}\left(\right.$ en $A_{R}{ }^{\prime \prime}$ ) se alinea con el vértice redondeado trasero en tanto que el $A$ (en $\mathrm{A}^{\prime \prime}$ ) se desplaza hacia abajo y se aleja del cuerpo.

Los torbellinos $\mathrm{C}$ y $\mathrm{C}_{\mathrm{R}}$ (en posición $\mathrm{C}^{\prime \prime}$ y $\mathrm{C}_{\mathrm{R}}$ "' respectivamente) continúan avanzando en sentido de la corriente.

$\theta=90^{\circ}$ : se observa el final del ciclo. Comienzan a generarse los nuevos torbellinos A, C (en E y D), $A_{R}$ y $C_{R}$ (en $E_{R} y D_{R}$ ) respectivos para cada cuerpo. El resto de los torbellinos continúan desplazándose arrastrados por la corriente. 


\section{R3 - Relación $u / v=0,36(\omega=1950 \mathrm{rpm}, v=10,2 \mathrm{~m} / \mathrm{s})$}

En la figura 3.23 se observa el comportamiento del flujo para el triángulo.
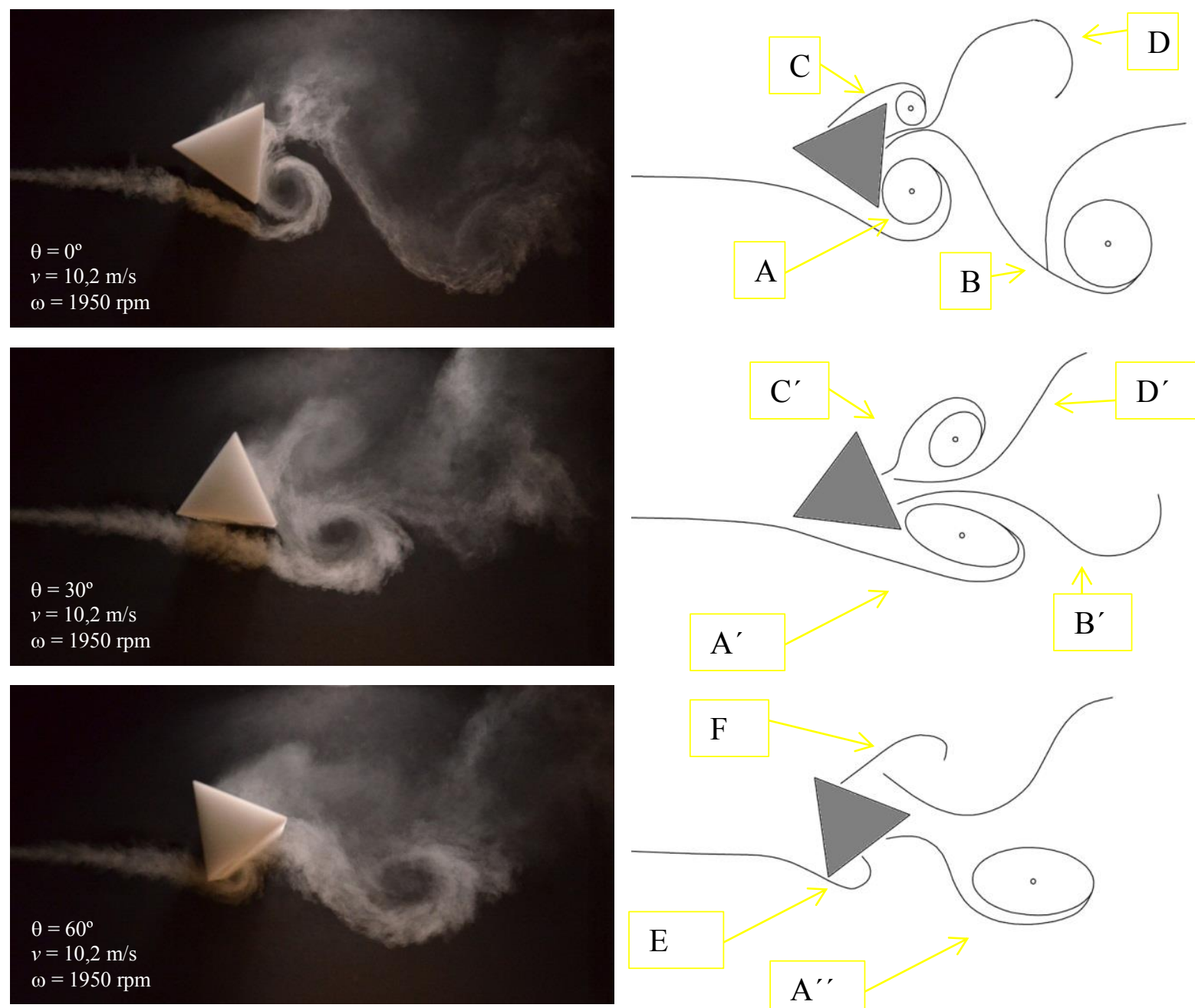

\section{$A^{\prime}$}

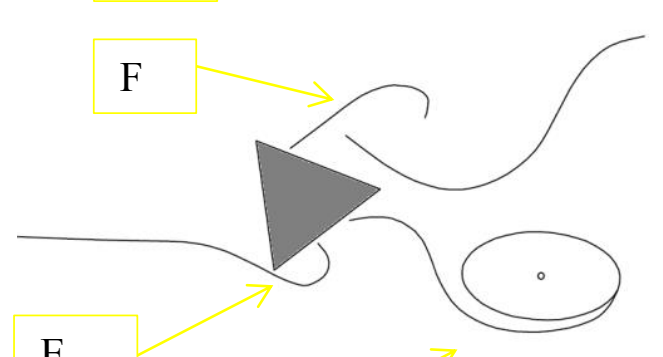

$\mathrm{E}$

$$
\mathrm{A}^{\prime \prime}
$$
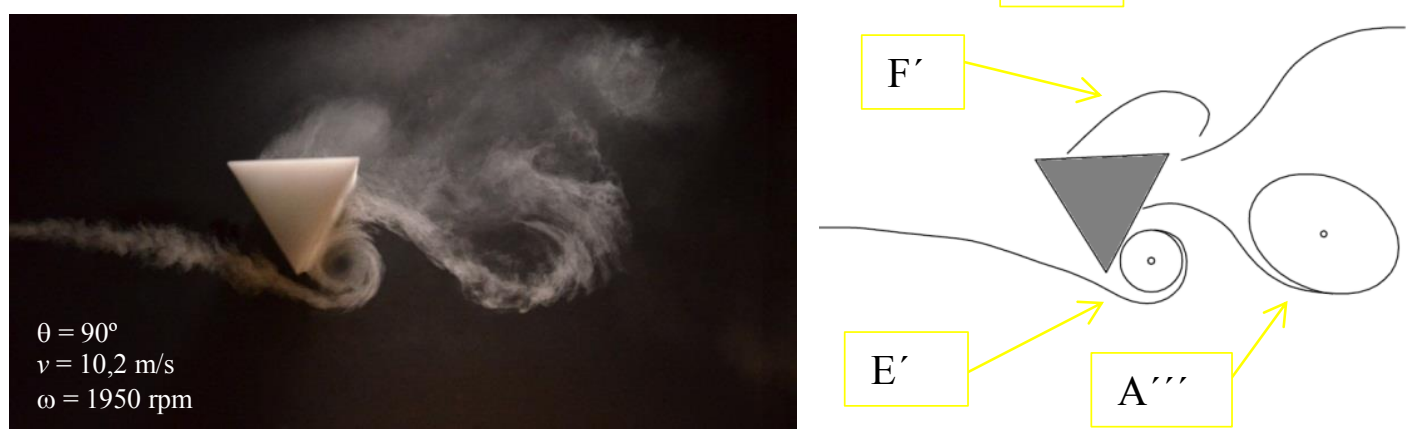

Figura 3.23 Configuración de flujo entorno al cuerpo en cada posición $\theta$. 
En la figura 3.24 se observa el comportamiento del flujo para el triángulo con aristas redondeadas.
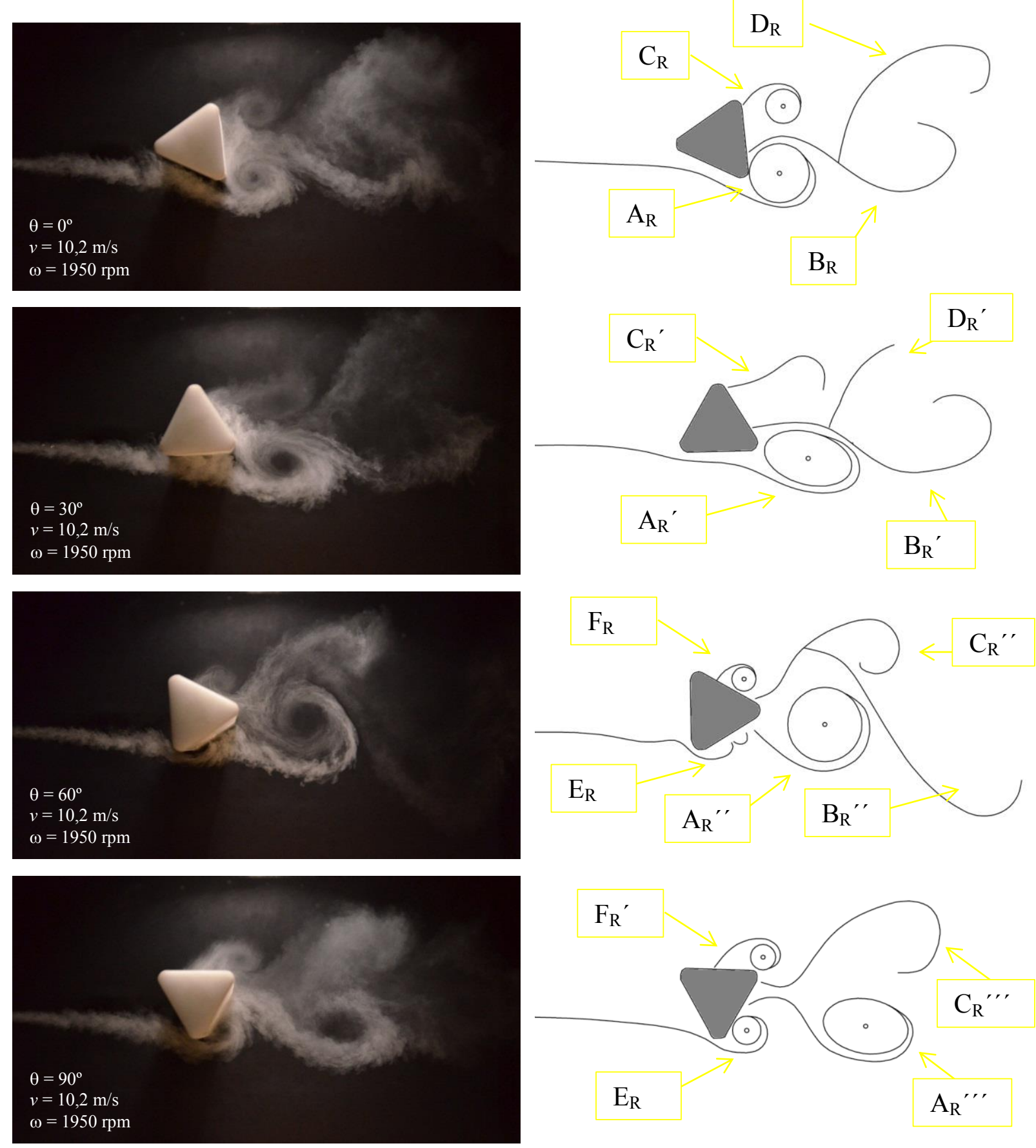

Figura 3.24 Configuración de flujo entorno al cuerpo en cada posición $\theta$. 
$\theta=0^{\circ}$ : el vértice agudo de triángulo genera un torbellino A más definido y de mayor tamaño que en caso del $A_{R}$. En tanto que el torbellino $B$ es de mayor tamaño y se encuentra más separado del cuerpo respecto al torbellino $\mathrm{B}_{\mathrm{R}}$ que se encuentra prácticamente desintegrado.

El torbellino $C$ es de mayor tamaño respecto del $C_{R}$, en tanto que los torbellinos $D$ y $D_{R}$ se encuentran en proceso de desintegración.

En la cara inferior el flujo se encuentra más adherido en el triángulo con bordes redondeados.

$\theta=30^{\circ}$ : los torbellinos $A\left(\right.$ en $\left.A^{\prime}\right), C\left(\right.$ en $\left.C^{\prime}\right), A_{R}\left(\right.$ en $\left.A_{R}{ }^{\prime}\right)$ y $C_{R}\left(\right.$ en $\left.C_{R}{ }^{\prime}\right)$ aumentan su tamaño y se separan del cuerpo. En el caso del triángulo, los mismos se separan más distancia que en el de bordes redondeados, a su vez que el A se desplaza hacia abajo en $\mathrm{A}^{\prime}$.

$\theta=60^{\circ}$ : el torbellino A (en $A^{\prime \prime}$ ), se desplaza hacia abajo y se aleja del cuerpo, en tanto que el torbellino $A_{R}\left(e n A_{R}{ }^{\prime \prime}\right)$ se alinea con el vértice redondeado trasero del cuerpo.

En el triángulo aparece un desprendimiento $\mathrm{F}$ en la parte superior. Así mismo en el triángulo con bordes redondeados comienza a formarse el torbellino $F_{R}$.

En ambos cuerpos se comienza a generar un desprendimiento $E$ y $E_{R}$ en el vértice inferior de los mismos.

$\theta=90^{\circ}$ : se observa el final del ciclo. Comienzan a generarse los nuevos torbellinos $A_{\text {y }} A_{R}$ (en $E^{\prime}$ y $\left.E_{R}{ }^{\prime}\right)$, y $C$ y $C_{R}$ (en $F^{\prime}$ y $F_{R}{ }^{\prime}$ ) respectivos para cada cuerpo.

Los torbellinos $\mathrm{C}$ y $\mathrm{C}_{\mathrm{R}}\left(\mathrm{en} \theta=0^{\circ}\right)$ se generan por efecto de la succión producida en la parte trasera del cuerpo que hace que el flujo se adhiera al mismo y luego se desprenda al tener menor energía que la corriente.

El resto de los torbellinos continúan desplazándose arrastrados por la corriente. 


\subsubsection{Conclusiones familia III}

La configuración de los flujos para el triángulo y el triángulo con aristas redondeadas es muy similar, sólo hay diferencia en los tamaños de los torbellinos (figuras 3.19 y 3.20).

La arista del triángulo es la encargada de generar torbellinos de mayor tamaño y junto con este un desprendimiento mayor del flujo, lo que se puede traducir en un aumento de la resistencia del cuerpo.

Se puede concluir observando las figuras 3.19 y 3.20 que en el caso del triángulo con arista afilada el torbellino se encuentra más alejado del cuerpo, disminuyendo su acción sobre la cara inferior, reduciendo la sustentación.

Así mismo se puede observar en el caso del triángulo con bordes redondeados la estela es más estrecha que en el otro cuerpo y por tal, disminuye su resistencia.

Al disminuir la relación de velocidades $u / v$ los torbellinos en ambos cuerpos comienzan a tener un tamaño más grande y los patrones tienden a asimilarse cada vez más, aunque los torbellinos son más grandes y en el triángulo están más separados por lo que los $C_{L}$ se equiparan pero la diferencia de $C_{D}$ se mantiene (anchura de la estela). 


\subsubsection{Familia IV}

La misma está conformada por la placa plana fina y la placa plana gruesa. En las figuras 3.25 y 3.26 se observa la variación de los coeficientes aerodinámicos $C_{L}$ y $C_{D}$ de los mencionados cuerpos con la variación de la relación de velocidades $u / v$. Los puntos $\mathrm{R}$ señalados en las mismas corresponden a diferentes condiciones de $u / v$ para las que se tomaron fotografías.

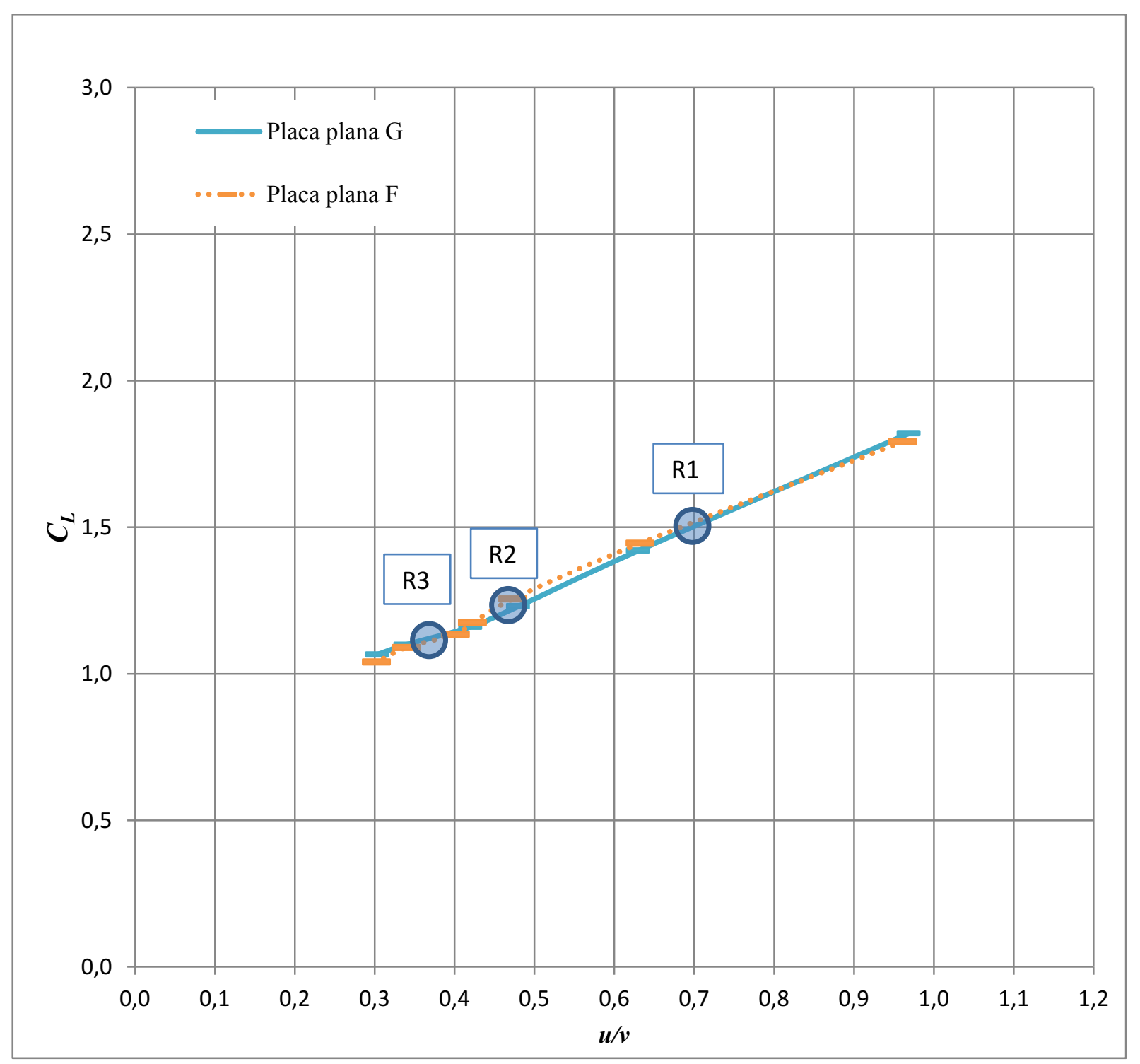

Figura 3.25 Variación del coeficiente de sustentación $C_{L}$ en función de la relación de velocidades $u / v$, y relaciones R1, R2 y R3 para el análisis fotográfico (círculos gruesos).

En el caso de la curva característica del $C_{L}$ (figura 3.25) no hay diferencias, sin embargo en el caso de $C_{D}$ (figura 3.26) se observan diferencias entre las dos placas, e incluso dos 
comportamientos diferentes en la placa plana gruesa, para una $\omega=1950 \mathrm{rpm}$ (a partir de $u / v=0,4)$ y otro para el resto de las velocidades de giro $\omega$. Se realiza un análisis particular al final de la descripción de la presente familia.

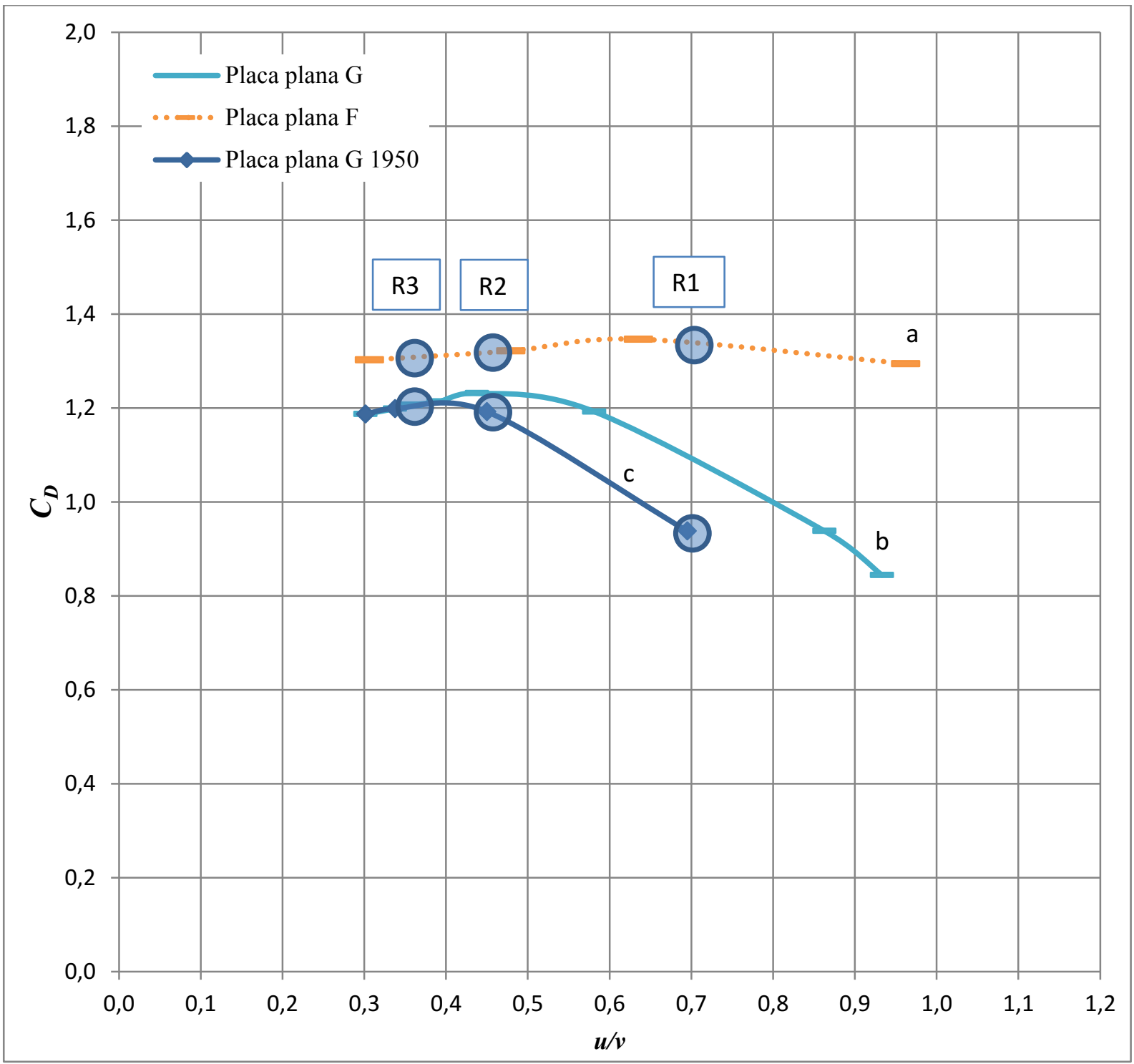

Figura 3.26 Variación del coeficiente de sustentación $C_{D}$ en función de la relación de velocidades $u / v$ y relaciones $\mathrm{R} 1, \mathrm{R} 2$ y R3 para el análisis fotográfico (círculos gruesos). Tres comportamientos (a (PPG), b (PPF) y c (PPG 1950 rpm).

A continuación se realiza una descripción de los flujos correspondientes a los diversos cuerpos de la Familia IV para las relaciones R1, R2 y R3. 


\section{$\mathrm{R} 1$ - Relación $u / v=0,70(\omega=1950 \mathrm{rpm}, v=5,2 \mathrm{~m} / \mathrm{s})$}

En la figura 3.27 se observa el comportamiento c del flujo para la placa plana gruesa.
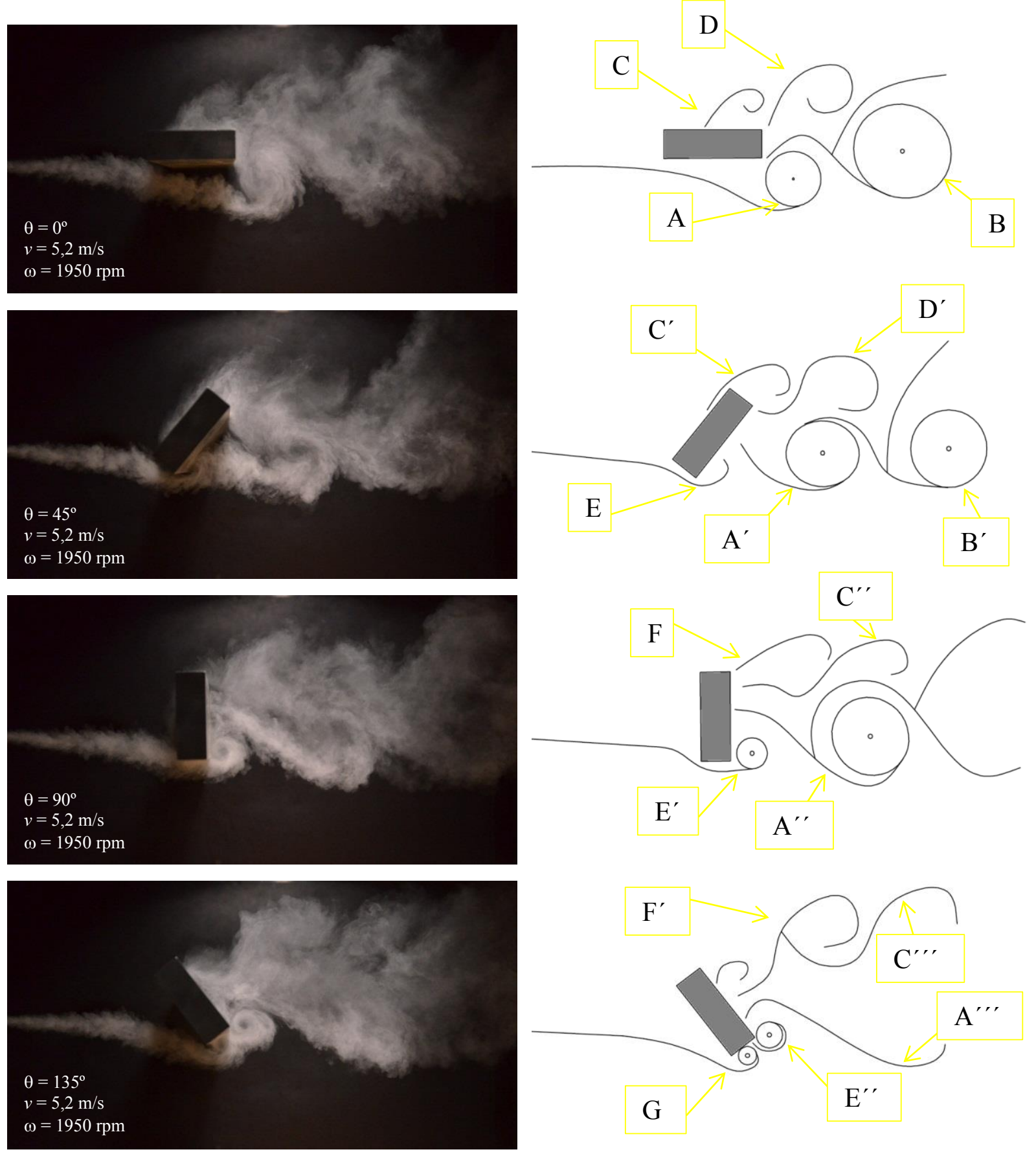

Figura 3.27 Configuración de flujo entorno al cuerpo en cada posición $\theta$. 
En la figura 3.28 se observa el comportamiento a del flujo para la placa plana fina.
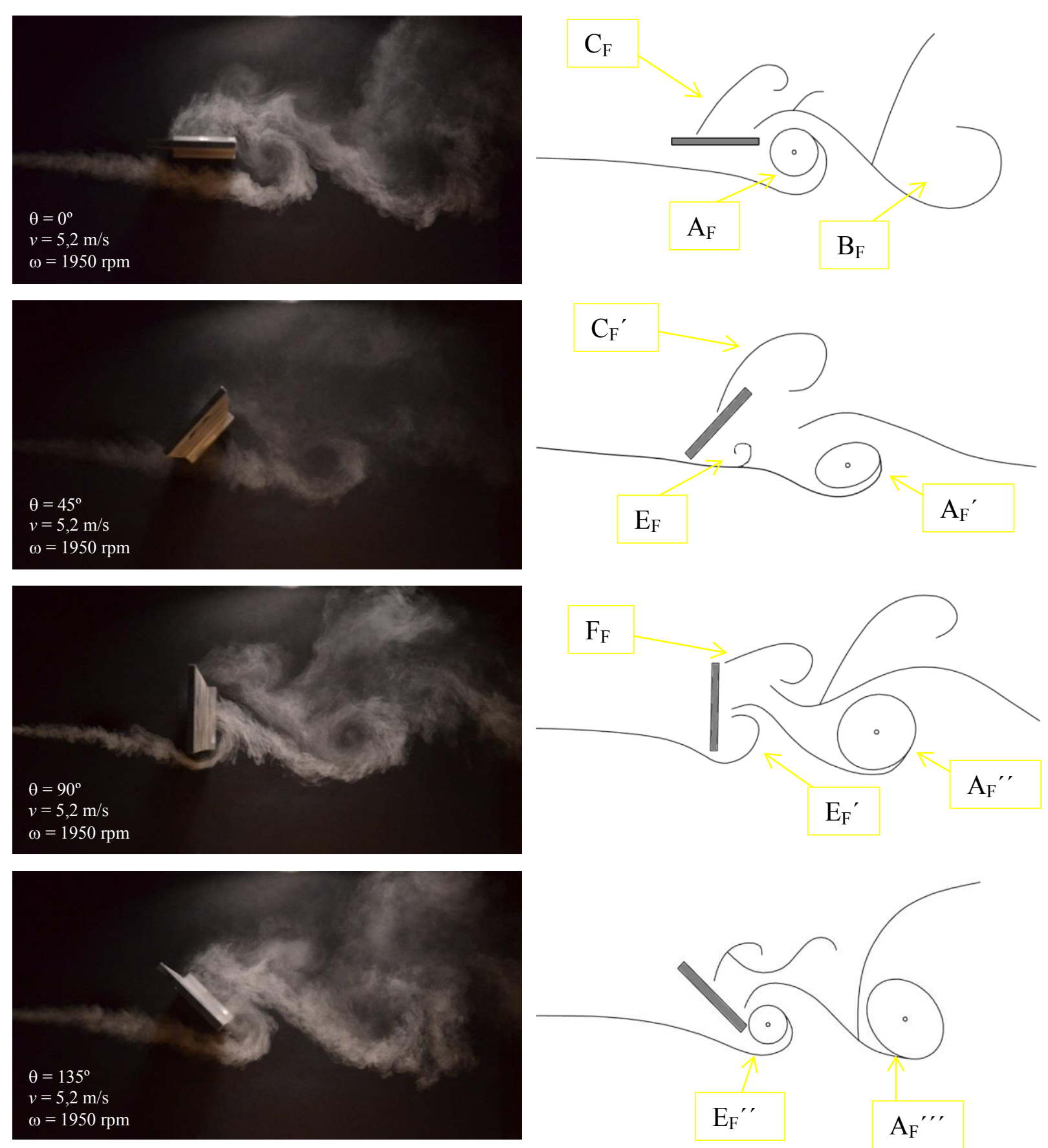

Figura 3.28 Configuración de flujo entorno al cuerpo en cada posición $\theta$. 
$\theta=0^{\circ}$ : los dos torbellinos A y $B$ en la placa plana gruesa y, un torbellino $A_{F}$ en la placa plana fina están bien definidos y han sido generados en el paso anterior, en tanto que el torbellino $\mathrm{B}_{\mathrm{F}}$ está en proceso de desintegración.

En la placa plana fina los torbellinos $A_{F}$ y $B_{F}$ poseen un diámetro algo mayor y se encuentran más separados que en la placa plana gruesa (torbellinos A y B).

Aparece flujo adherido en la cara superior $\mathrm{C}$ y $\mathrm{C}_{\mathrm{F}}$ en ambos cuerpos. Comienza el desprendimiento de ambos.

El torbellino D está en proceso de desintegración en la placa plana gruesa.

$\theta=45^{\circ}$ : comienza la generación de flujo desprendido $\mathrm{E}$ y $\mathrm{E}_{\mathrm{F}}$ en las aristas afiladas inferiores de cada cuerpo.

Los torbellinos $\mathrm{A}, \mathrm{B}, \mathrm{A}_{\mathrm{F}}$ y $\mathrm{B}_{\mathrm{F}}$ continúan desplazándose corriente abajo a las posiciones $\mathrm{A}^{\prime}, \mathrm{B}^{\prime}$, $\mathrm{A}_{\mathrm{F}}{ }^{\prime}$ y $\mathrm{B}_{\mathrm{F}}{ }^{\prime}$ respectivamente.

En ambos casos el flujo adherido del paso anterior está casi desprendido en la cara superior, ahora en posición $\mathrm{C}^{\prime}$ y $\mathrm{C}_{\mathrm{F}}{ }^{\prime}$.

$\theta=90^{\circ}$ : comienzan a desarrollarse los torbellinos $\mathrm{E}^{\prime}$ y $\mathrm{E}_{\mathrm{F}}{ }^{\prime}$, en tanto que los torbellinos $\mathrm{A}$ (en $\left.\mathrm{A}^{\prime \prime}\right)$ y $\mathrm{A}_{\mathrm{F}}\left(\mathrm{en}_{\mathrm{F}}{ }^{\prime \prime}\right)$ continúan desplazándose corriente abajo.

Hay flujo desprendiéndose en $\mathrm{F}_{\text {y }} \mathrm{F}_{\mathrm{F}}$ en el borde superior en ambos cuerpos, en tanto que los

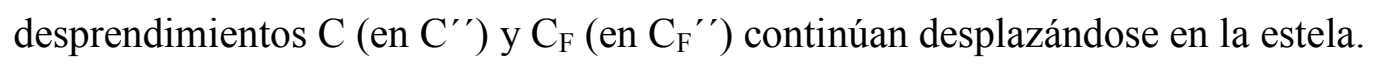

$\theta=135^{\circ}$ : continúa el desarrollo de los torbellinos A (en $\left.A^{\prime \prime \prime}\right)$ y $F\left(e n F^{\prime}\right)$. Se genera el torbellino $\mathrm{G}$ debido al espesor de la placa plana gruesa, que en conjunción con el torbellino $\mathrm{E}$ (en $\mathrm{E}^{\prime \prime}$ ) son los precursores del torbellino A para $\theta=0^{\circ}$, en tanto que el torbellino A (en $\mathrm{A}^{\prime \prime}$ ) comienza a desintegrarse. Para el caso de la placa plana fina no aparece un torbellino nuevo y continúan desarrollándose el $\mathrm{E}_{\mathrm{F}}\left(\right.$ en $\mathrm{E}_{\mathrm{F}}{ }^{\prime \prime}$ ) y el $\mathrm{A}_{\mathrm{F}}\left(\right.$ en $\mathrm{A}_{\mathrm{F}}{ }^{\prime \prime \prime}$ ) (que en posición $\theta=0^{\circ}$ es el torbellino $\mathrm{B}_{\mathrm{F}}$ ).

Aparece flujo remansado en la cara trasera de ambos cuerpos. 


\section{R2 - Relación $u / v=0,47(\omega=1950 \mathrm{rpm}, v=7,7 \mathrm{~m} / \mathrm{s})$}

En la figura 3.29 se observa el comportamiento c del flujo para la placa plana gruesa.
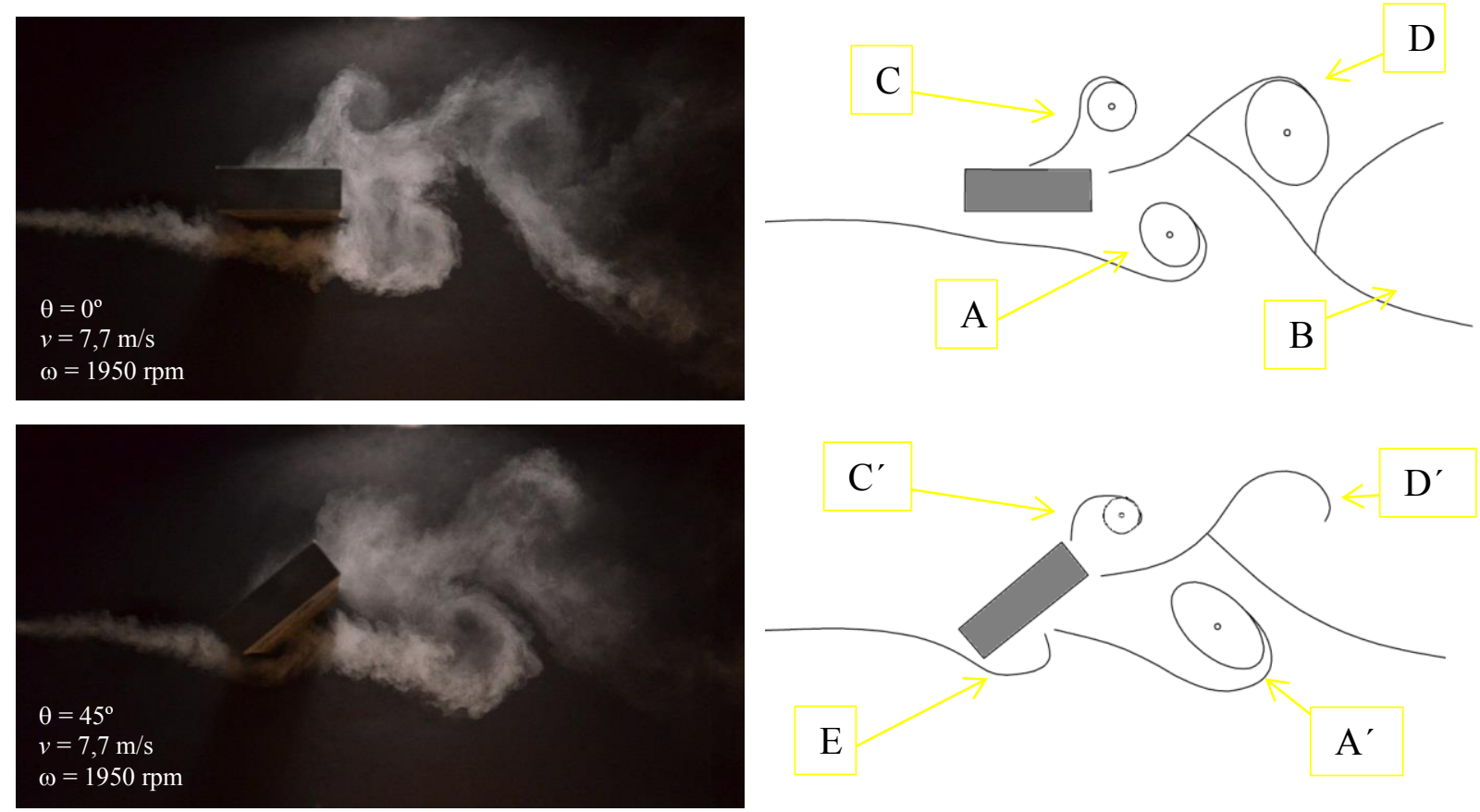

$v=7,7 \mathrm{~m} / \mathrm{s}$

$\mathrm{E}$

$\mathrm{A}^{\prime}$
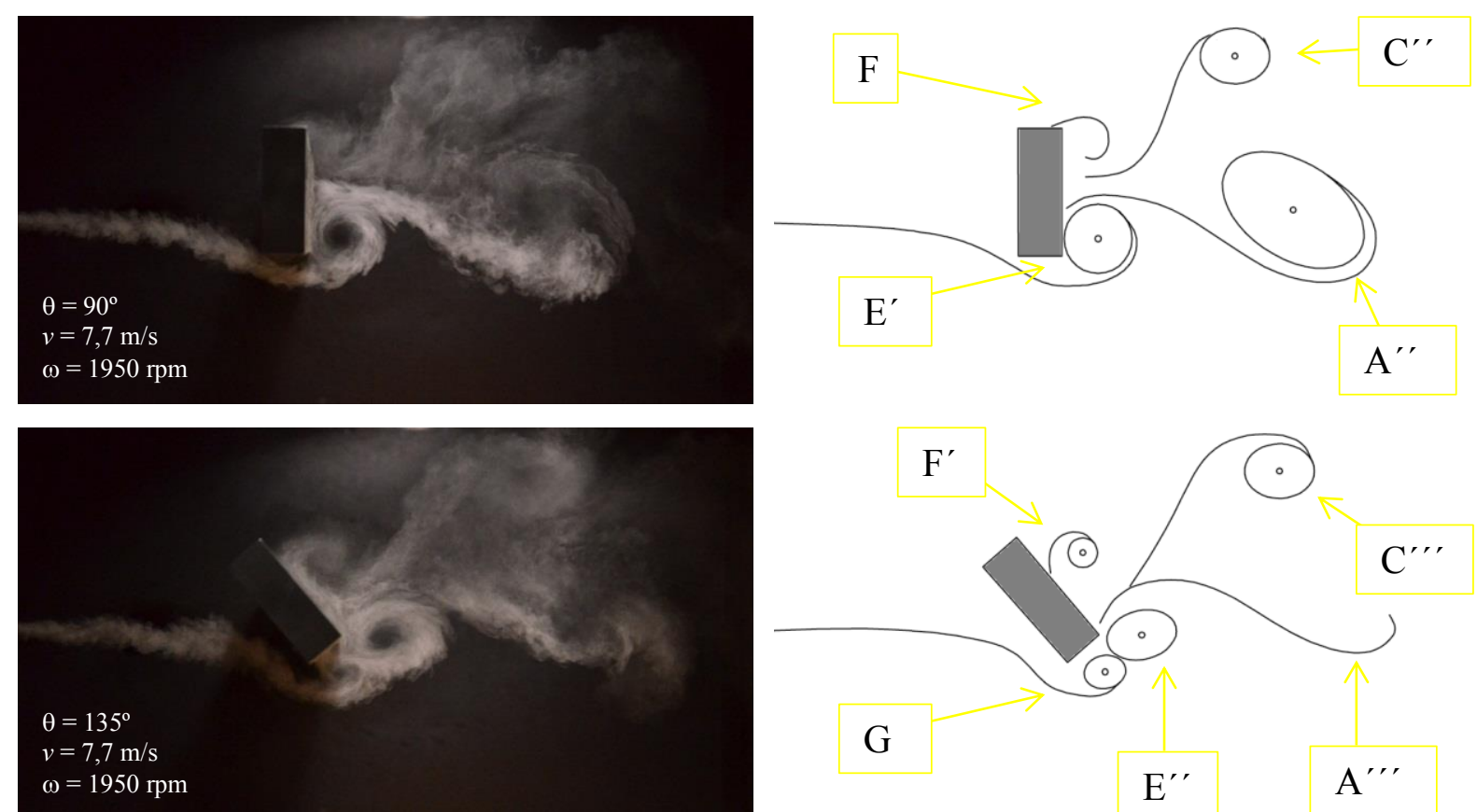

Figura 3.29 Configuración de flujo entorno al cuerpo en cada posición $\theta$. 
En la figura 3.30 se observa el comportamiento a del flujo para la placa plana fina.
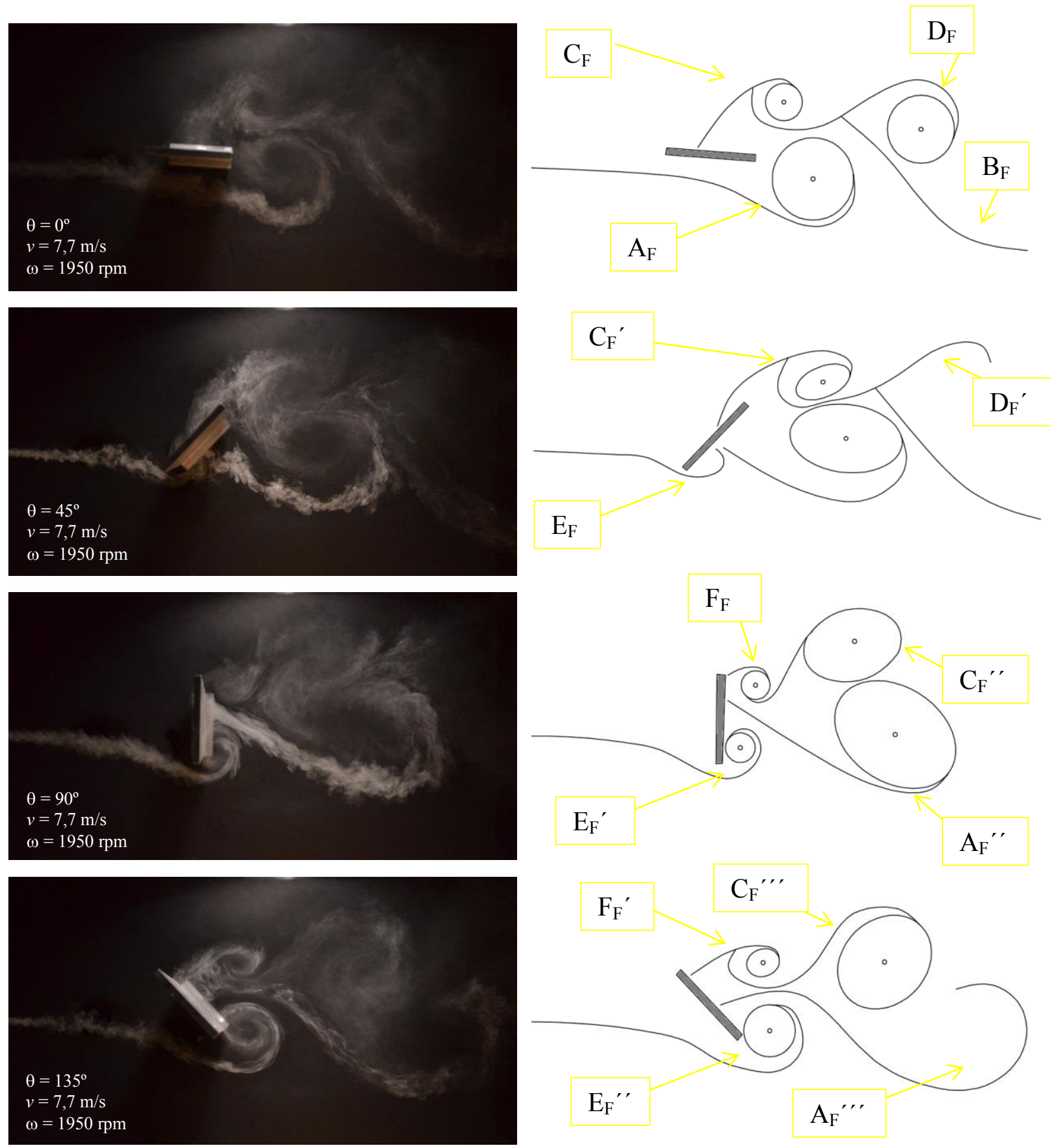

Figura 3.30 Configuración de flujo entorno al cuerpo en cada posición $\theta$. 
$\theta=0^{\circ}$ : los torbellinos A y $A_{F}$ están bien definidos. El torbellino $A_{F}$ es de mayor tamaño que el torbellino A y se encuentra más separado del cuerpo.

Las líneas de flujo $\mathrm{B}$ y $\mathrm{B}_{\mathrm{F}}$ son producto de la desintegración de torbellinos de ciclos anteriores.

Los torbellinos $\mathrm{C}$ y $\mathrm{C}_{\mathrm{F}}$ con sentido de giro horario son el resultado del desprendimiento del flujo adherido en la cara superior del cuerpo.

Los torbellinos $\mathrm{D}$ y $\mathrm{D}_{\mathrm{F}}$ con sentido de giro horario son los sucesores de los torbellinos $\mathrm{C}$ y $\mathrm{C}_{\mathrm{F}}$, respectivamente desprendidos del cuerpo en el tramo del giro anterior.

En la cara inferior de la placa plana fina el flujo se encuentra más separado que en el otro cuerpo.

$\theta=45^{\circ}$ : los torbellinos $\mathrm{A}$ (en $\left.\mathrm{A}^{\prime}\right), \mathrm{C}\left(\right.$ en $\left.\mathrm{C}^{\prime}\right), \mathrm{A}_{\mathrm{F}}\left(\right.$ en $\left.\mathrm{A}_{\mathrm{F}}{ }^{\prime}\right)$ y $\mathrm{B}_{\mathrm{F}}\left(\right.$ en $\left.\mathrm{B}_{\mathrm{F}}{ }^{\prime}\right)$ están siendo desplazados corriente abajo. Los torbellinos $A_{F}\left(\right.$ en $\left.A_{F}{ }^{\prime}\right)$ y $C_{F}\left(\right.$ en $\left.C_{F}{ }^{\prime}\right)$ son de mayor tamaño y están más desplazados hacia atrás que los respectivos en $\mathrm{A}^{\prime} \mathrm{y}^{\prime}$.

El torbellino $C$ (en $C^{\prime}$ ) está en desarrollo, en tanto el torbellino $\mathrm{C}_{\mathrm{F}}\left(\right.$ en $\left._{\mathrm{F}}{ }^{\prime}\right)$ continúa creciendo y desplazándose corriente abajo.

Los torbellinos $\mathrm{D}$ y $\mathrm{D}_{\mathrm{F}}$ de $\theta=0^{\circ}$ aparecen en proceso de desintegración ( $\mathrm{D}^{\prime}$ y $\left.\mathrm{D}_{\mathrm{F}}{ }^{\prime}\right)$.

En ambos cuerpos comienza un desprendimiento en $\mathrm{E}$ y $\mathrm{E}_{\mathrm{F}}$ en el borde inferior.

Hay flujo adherido en la cara superior de ambos cuerpos.

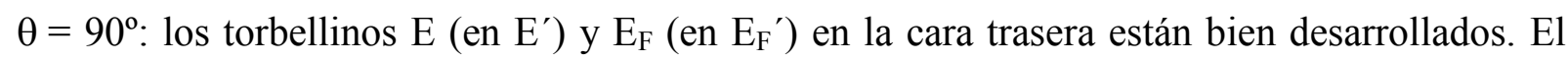
torbellino $\mathrm{E}^{\prime}$ es de mayor tamaño y más definido que el torbellino $\mathrm{E}_{\mathrm{F}}{ }^{\prime}$.

La línea de flujo del torbellino $\mathrm{A}_{\mathrm{F}}{ }^{\prime \prime}$ comienza en una posición vertical más elevada sobre la cara trasera del cuerpo que la del torbellino A".

El torbellino $A_{F}$ (ahora en $A_{F}{ }^{\prime \prime}$ ) está más alejado del cuerpo que el torbellino $A$ (en $A^{\prime \prime}$ ).

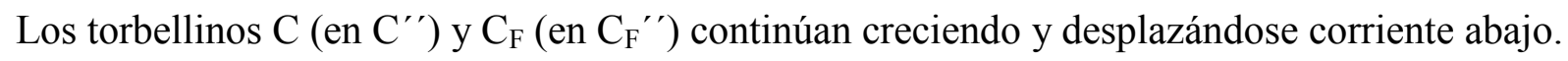


$\theta=135^{\circ}:$ los torbellinos $\mathrm{E}\left(\mathrm{en}^{\prime \prime}\right)$ y $\mathrm{E}_{\mathrm{F}}\left(\mathrm{en}_{\mathrm{F}}{ }^{\prime \prime}\right)$ están bien definidos. El torbellino $\mathrm{E}_{\mathrm{F}}{ }^{\prime \prime}$ es de mayor tamaño, más definido y más desplazado hacia abajo que el torbellino $\mathrm{E}^{\prime \prime}$.

Se genera el torbellino $\mathrm{G}$ debido al espesor de la placa plana gruesa que en conjunción con el torbellino $\mathrm{E}$ (en $\mathrm{E}^{\prime \prime}$ ) son los generadores del torbellino A para $\theta=0^{\circ}$, en tanto que el torbellino A (en $\left.\mathrm{A}^{\prime \prime \prime}\right)$ comienza a desintegrarse.

Para el caso de la placa plana fina no aparece un torbellino nuevo y continúa desarrollándose el torbellino $\mathrm{E}_{\mathrm{F}}\left(\right.$ en $\mathrm{E}_{\mathrm{F}}{ }^{\prime \prime \prime}$ ), en tanto que el $\mathrm{A}_{\mathrm{F}}\left(\right.$ en $\mathrm{A}_{\mathrm{F}}{ }^{\prime \prime \prime}$ ) (en la siguiente posición $\theta=0^{\circ}$ es el torbellino $\mathrm{B}_{\mathrm{F}}$ ) comienza a desintegrarse.

Así mismo, los torbellinos $\mathrm{C}$ (en $\left.\mathrm{C}^{\prime \prime \prime}\right)$ y $\mathrm{C}_{\mathrm{F}}\left(\right.$ en $\left._{\mathrm{F}_{\mathrm{F}}}{ }^{\prime \prime \prime}\right)$ continúan desplazándose corriente abajo (en $\theta=0^{\circ}$ son los torbellinos $\mathrm{D}$ y $\mathrm{D}_{\mathrm{F}}$ respectivamente).

Los torbellinos $\mathrm{F}$ (en $\mathrm{F}^{\prime}$ ) y $\mathrm{F}_{\mathrm{F}}\left(\right.$ en $\mathrm{F}_{\mathrm{F}}{ }^{\prime}$ ) están bien definidos y son el producto del desprendimiento del flujo adherido en la cara superior. 


\section{R3 - Relación $u / v=0,36(\omega=1950 \mathrm{rpm}, v=10,2 \mathrm{~m} / \mathrm{s})$}

En la figura 3.31 se observan los comportamientos b y c (ambos coinciden para $u / v<0,4)$ del flujo para la placa plana gruesa.
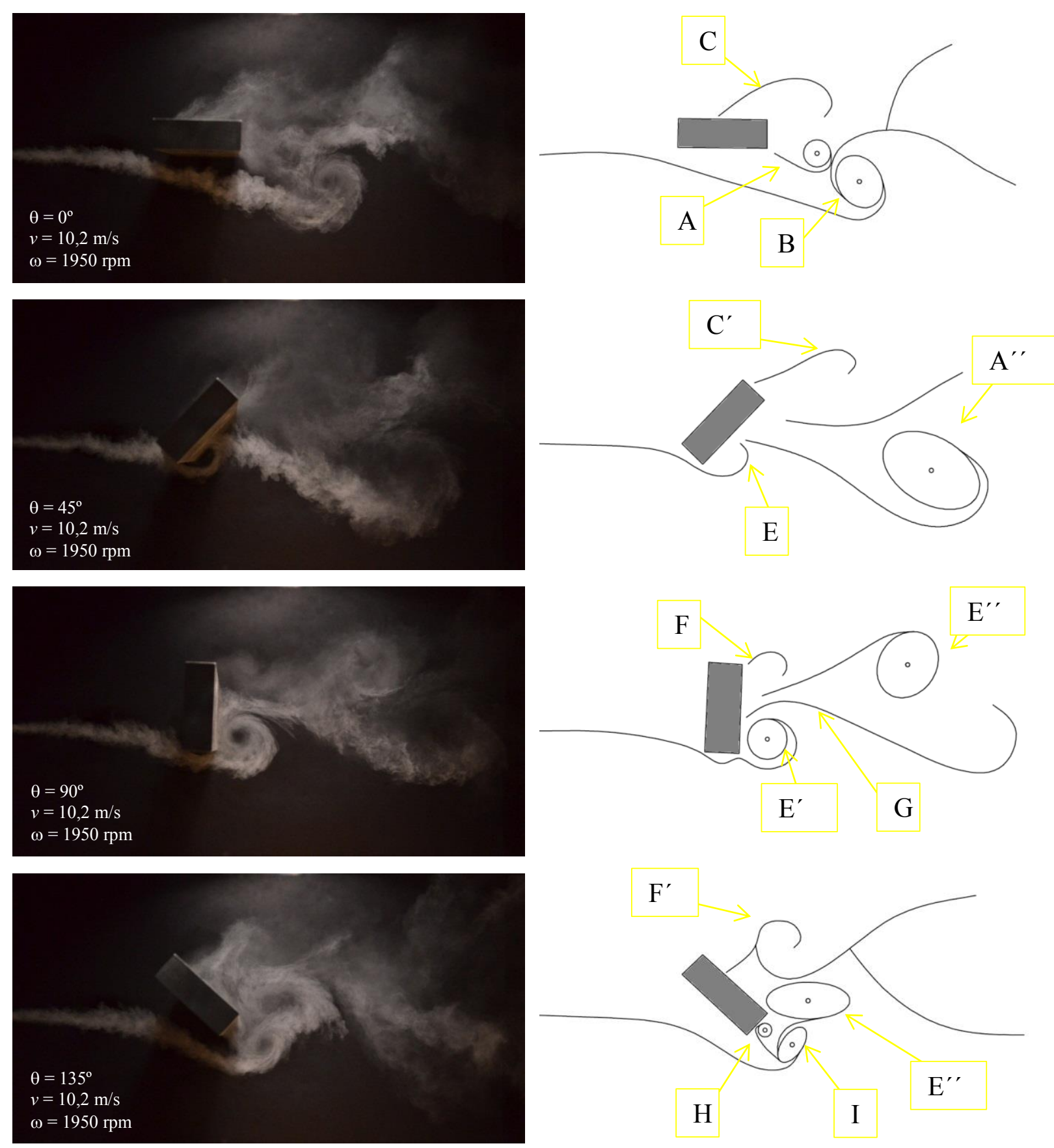

Figura 3.31 Configuración de flujo entorno al cuerpo en cada posición $\theta$. 
En la figura 3.32 se observa el comportamiento a, del flujo para la placa plana fina.
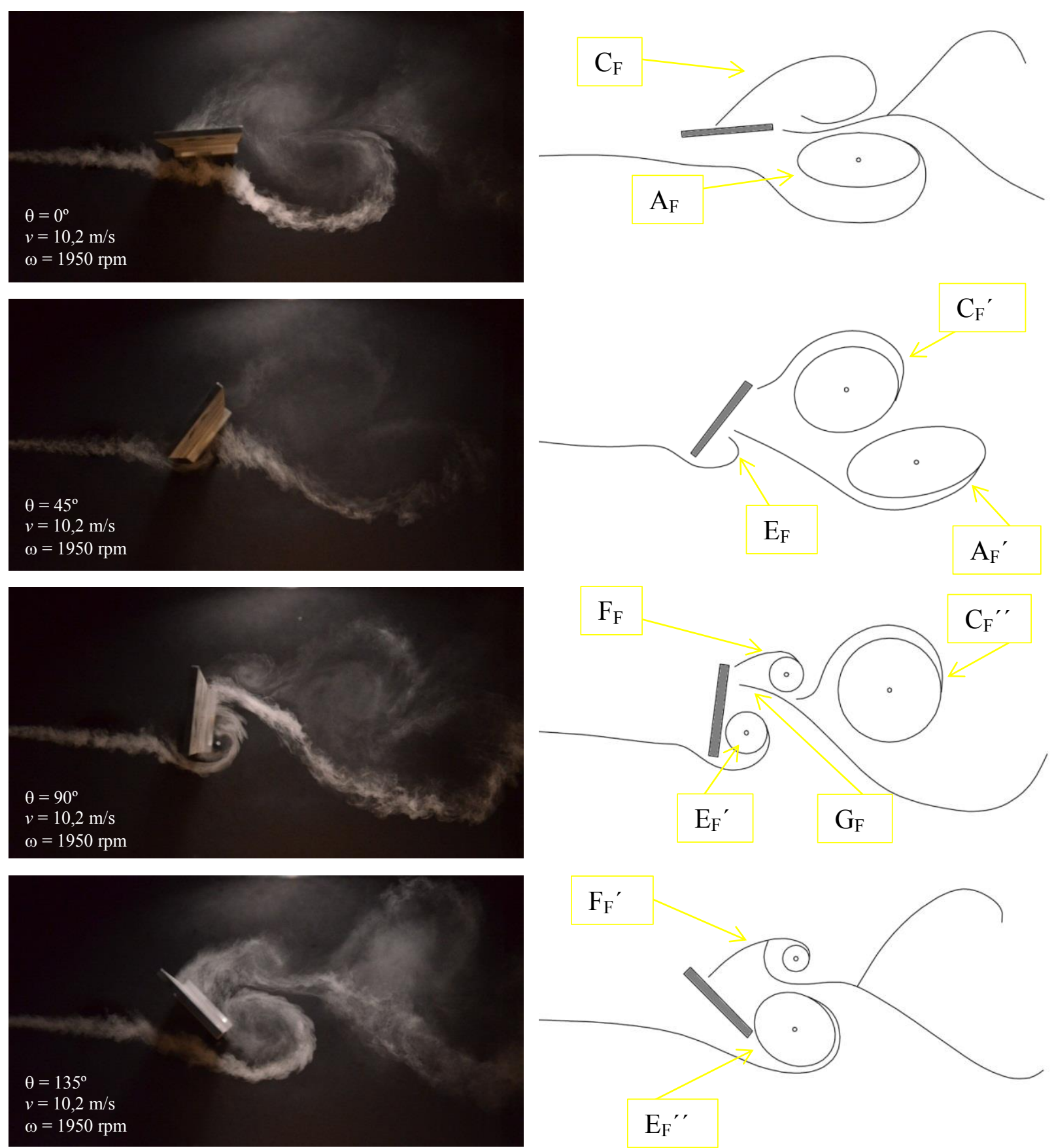

Figura 3.32 Configuración de flujo entorno al cuerpo en cada posición $\theta$. 
$\theta=0^{\circ}$ : aparecen dos torbellinos bien definidos A y B en la placa plana gruesa y un sólo torbellino $\mathrm{A}_{\mathrm{F}}$ de gran tamaño en la placa plana fina.

El flujo $\mathrm{C}$ y $\mathrm{C}_{\mathrm{F}}$ empieza a desprenderse en la cara superior de ambos cuerpos.

$\theta=45^{\circ}$ : comienzo de desprendimiento $\mathrm{E}$ y $\mathrm{E}_{\mathrm{F}}$ en la arista inferior.

En la placa plana gruesa, los torbellinos A y $\mathrm{B}\left(\theta=0^{\circ}\right)$ se mezclan generando un sólo torbellino en $\mathrm{A}^{\prime \prime}$.

En la placa plana fina se genera el torbellino $\mathrm{C}_{\mathrm{F}}{ }^{\prime}$ producto del desprendimiento $\mathrm{C}_{\mathrm{F}}$ del paso anterior.

$\theta=90^{\circ}$ : formación de torbellinos $\mathrm{E}^{\prime} \mathrm{y}_{\mathrm{F}}{ }_{\mathrm{F}}$ bien definidos en la parte inferior de la cara trasera.

Los torbellinos $\mathrm{F}$ y $\mathrm{F}_{\mathrm{F}}$ en la parte superior de los cuerpos, producto del desprendimiento del flujo adherido en cara superior.

Torbellinos $\mathrm{C}$ (en $\mathrm{C}^{\prime \prime}$ ) y $\mathrm{C}_{\mathrm{F}}\left(\mathrm{en}_{\mathrm{F}}{ }^{\prime \prime}\right.$ ) bien definidos formando parte de la estela. El torbellino $\mathrm{C}_{\mathrm{F}}{ }^{\prime \prime}$ de mayor tamaño que el torbellino $\mathrm{C}^{\prime \prime}$.

Los torbellinos $\mathrm{A}^{\prime \prime}$ y $\mathrm{A}_{\mathrm{F}}{ }^{\prime \prime}$ del paso anterior están en proceso de desintegración.

El inicio de la línea de flujo $G_{F}$ está en una posición vertical más elevada que la línea $\mathrm{G}$.

$\theta=135^{\circ}:$ se produce la formación de dos torbellinos $\mathrm{H}$ e I en el espesor de la placa plana gruesa, que desplazan hacia arriba al torbellino $\mathrm{E}$ (en $\mathrm{E}^{\prime \prime}$ ).

En la placa plana fina los torbellinos $\mathrm{E}_{\mathrm{F}}\left(\mathrm{en}_{\mathrm{F}}{ }^{\prime \prime}\right)$ y $\mathrm{F}_{\mathrm{F}}\left(\right.$ en $\left.\mathrm{F}_{\mathrm{F}}{ }^{\prime}\right)$ continúan desplazándose corriente abajo.

Los torbellinos $\mathrm{H}$ e I se transforman en el torbellino A del paso $\theta=0^{\circ}$. En tanto que los torbellinos $\mathrm{E}_{\mathrm{F}}{ }^{\prime \prime} \mathrm{y} \mathrm{F}_{\mathrm{F}}{ }^{\prime}$ de la placa plana fina pasan a ser los torbellinos $\mathrm{A}_{\mathrm{F}} \mathrm{y} \mathrm{C}_{\mathrm{F}}$ en $\theta=0^{\circ}$ respectivamente. 


\subsubsection{Conclusiones familia IV}

Los patrones de flujo son similares en ambos cuerpos. Se destaca el hecho de que la placa plana gruesa tenga un espesor considerable, da lugar a que se generen torbellinos pequeños que en la placa plana fina no se producen. Sin embargo, este hecho no influye en la curva característica de $C_{L}$.

Todos los torbellinos (superiores e inferiores) que se generan en la placa plana fina son de mayor tamaño que los que se generan en la placa plana gruesa. Así mismo, se encuentran más separados del cuerpo desde que se forman hasta que se desprenden.

Al aumentar la relación de velocidades $u / v$ en la placa plana fina los torbellinos aumentan su tamaño y a su vez aumenta el ancho de la estela, en tanto que en la placa plana gruesa al aumentar la relación de velocidades el ancho de la estela disminuye.

Estas razones explican la diferencia que existe entre los coeficientes de resistencia $C_{D}$ de un cuerpo respecto al otro.

La diferencia en el comportamiento $\mathrm{c}$ de la placa plana gruesa a $\omega=1950 \mathrm{rpm}$ respecto al resto de las velocidades de rotación $\omega$ (comportamiento b), radica en el tamaño de los torbellinos generados. Si bien la forma del patrón es la misma para cada velocidad de rotación $\omega$, el tamaño de los torbellinos que se generan a $\omega=1950 \mathrm{rpm}$ es mucho menor que para el resto de las velocidades de giro.

Se observa que la curva c, para dicha condición de giro posee la misma forma que para el resto (curva b), sólo que la caída en el $C_{D}$ ocurre para una relación $u / v$ menor. En el caso de la placa fina, la relación $u / v$ influye muy poco en la variación del $C_{D}$ (figura 3.26). 


\subsubsection{Familia V}

La misma está conformada por el cuerpo circular liso, el circular recubierto con lija fina, el circular recubierto con lija gruesa y el circular calado. En las figuras 3.33 y 3.34 se observa la variación de los coeficientes aerodinámicos $C_{L}$ y $C_{D}$ de los mencionados cuerpos con la relación de velocidades $u / v$. Los puntos $\mathrm{R}$ señalados en las mismas corresponden a diferentes relaciones de $u / v$ para las que se tomaron fotografías. Para dichos casos se realiza un estudio de las mismas con el objeto de determinar, el comportamiento del flujo alrededor de los cuerpos.

En las curvas características de $C_{L}$ para esta familia se observan tres comportamientos (a, b y c). Así mismo se identifican dos comportamientos para el circular recubierto con lija gruesa, uno para una velocidad de giro $\omega=1950 \mathrm{rpm}$ (comportamiento c) y otro comportamiento para el resto de las velocidades de giro (comportamiento $b$ ).

En la curva característica de $C_{D}$ también se observan tres comportamientos (a, b y c). Así mismo se identifican dos comportamientos para el circular calado. Se observa un comportamiento diferente para una velocidad de giro $\omega=2725 \mathrm{rpm}$ (comportamiento b) y otro para el resto.

Estas diferencias en los comportamientos se describen al final en las conclusiones correspondientes a la familia en estudio. 


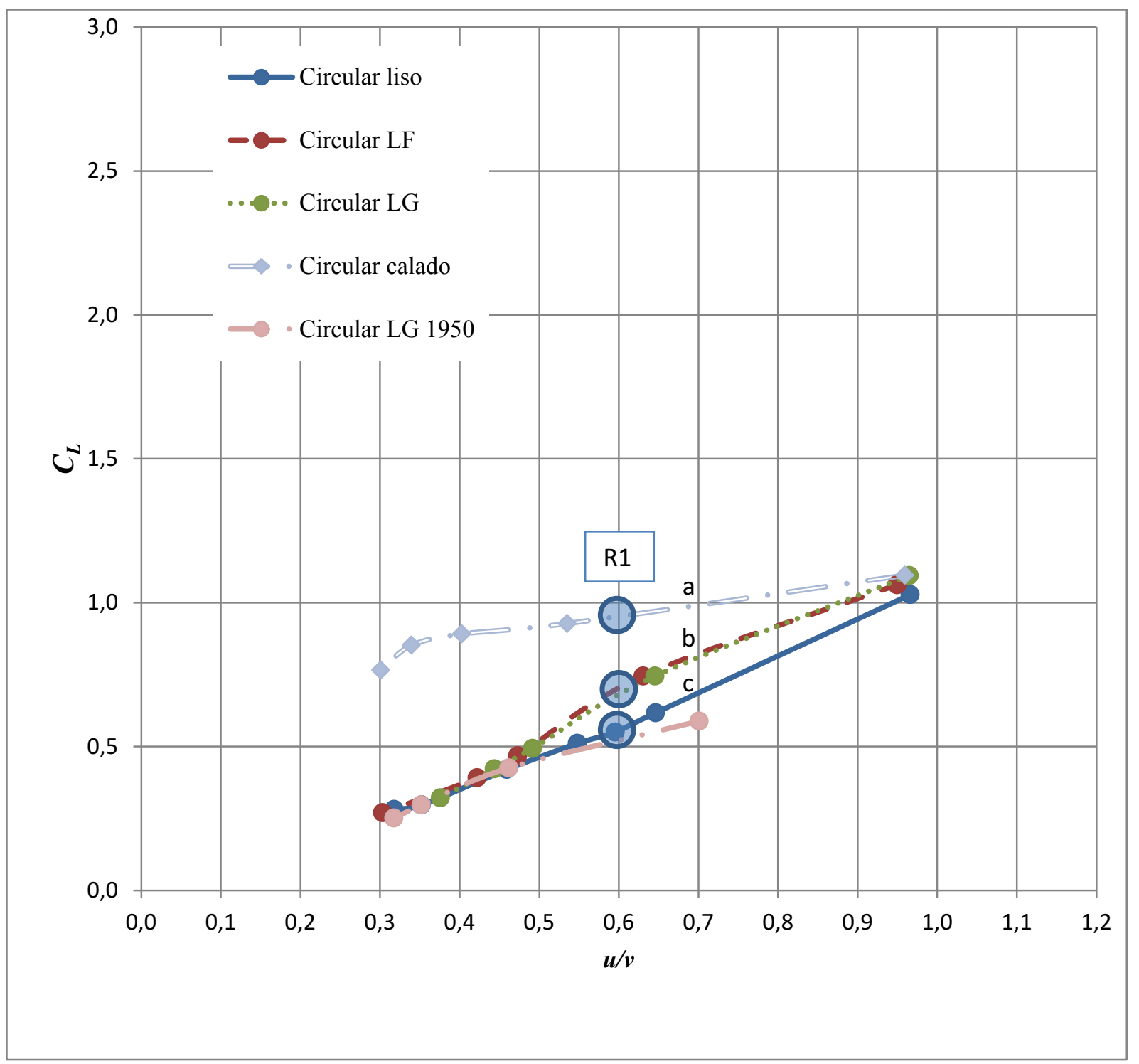

Figura 3.33 Variación del coeficiente de sustentación $C_{L}$ en función de la relación de velocidades $u / v$ y relación R1 para el análisis fotográfico (círculos gruesos). Tres comportamientos (a (CC), b (CLG, CLF) y c (CL, CLG1950 rpm). 


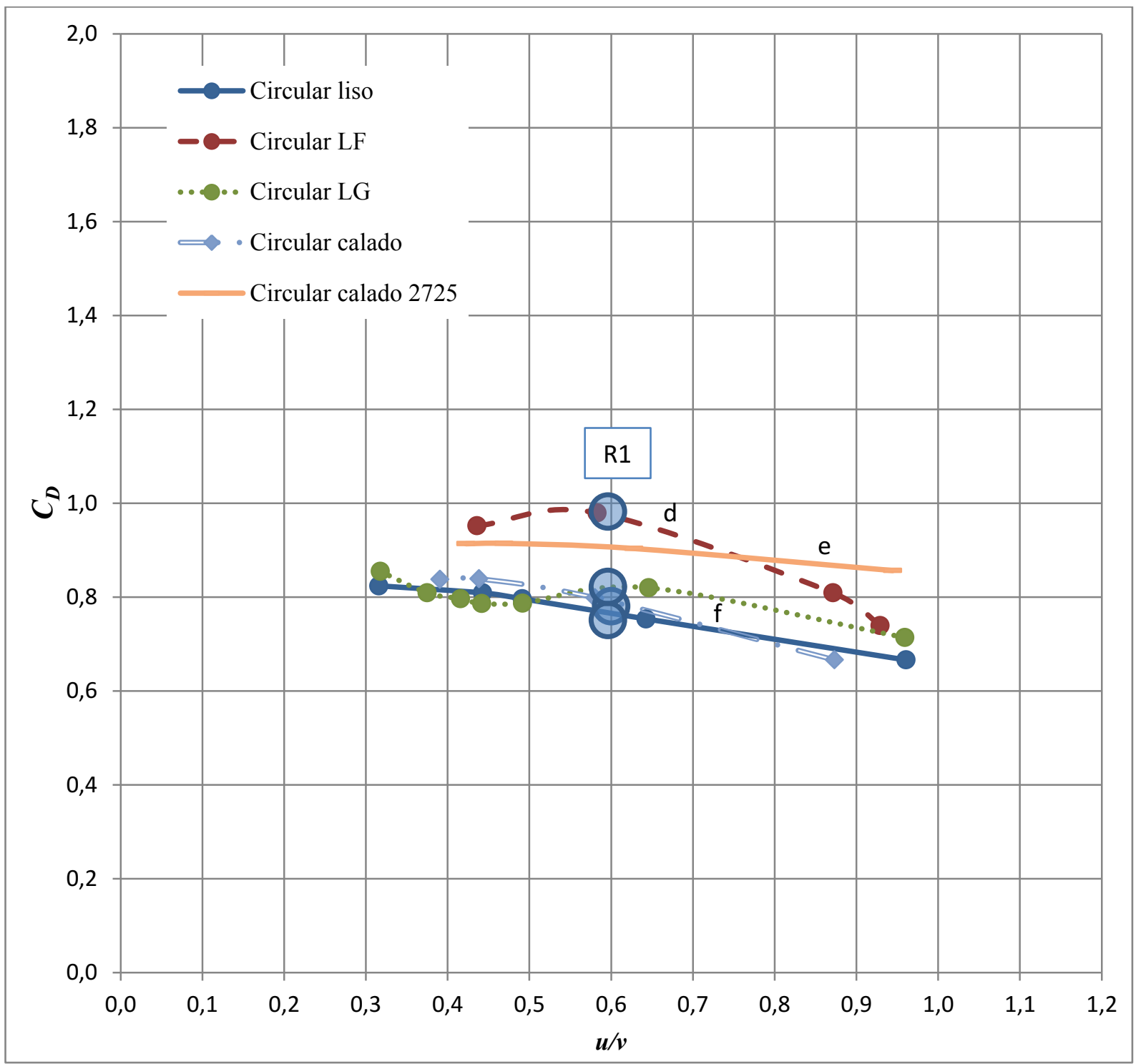

Figura 3.34 Variación del coeficiente de sustentación $C_{D}$ en función de la relación de velocidades $u / v$ y relación $\mathrm{R} 1$ para el análisis fotográfico (círculos gruesos). Tres comportamientos (d (CLF), e (CC2725 rpm) y f(CL, CC, CLG)).

A continuación se realiza una descripción de los flujos correspondientes a los diversos cuerpos de la familia V para la relación R1. 


\section{R1 - Relación $u / v=0,60(\omega=2520 \mathrm{rpm}, v=7,7 \mathrm{~m} / \mathrm{s})$}

La figura 3.35 se corresponde con el comportamiento c del flujo alrededor del cilindro circular liso.
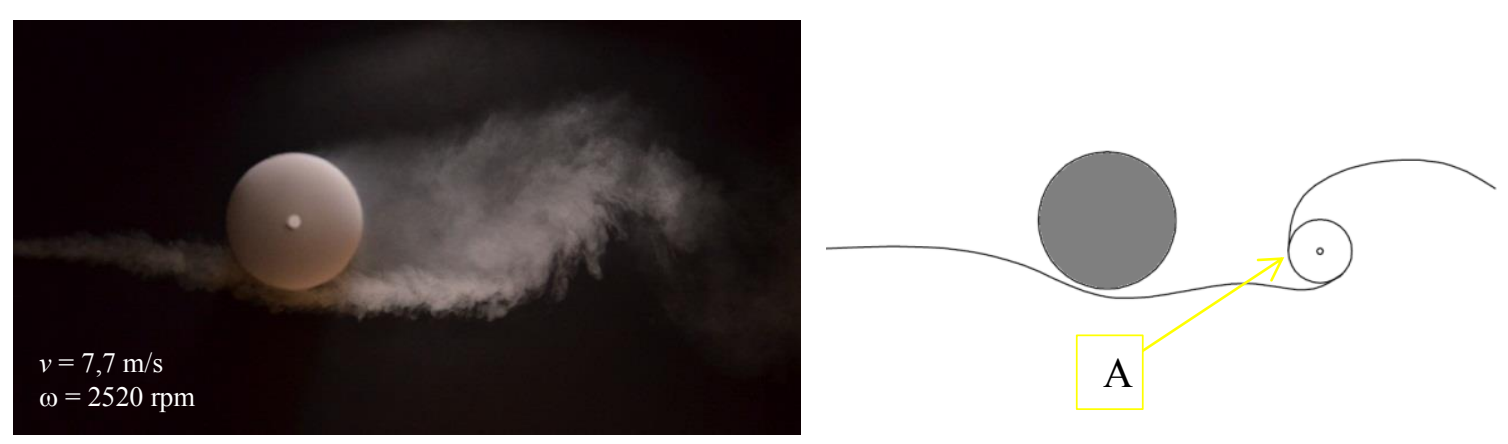

Figura 3.35 Configuración del flujo entorno al cuerpo.

Se genera un torbellino A que al no tener medida de la fase (los torbellinos no están sincronizados con una arista como los otros casos) no se puede determinar con precisión la ubicación del mismo.

La figura 3.36 se corresponde con el comportamiento b o d del flujo alrededor del cilindro circular con lija fina.
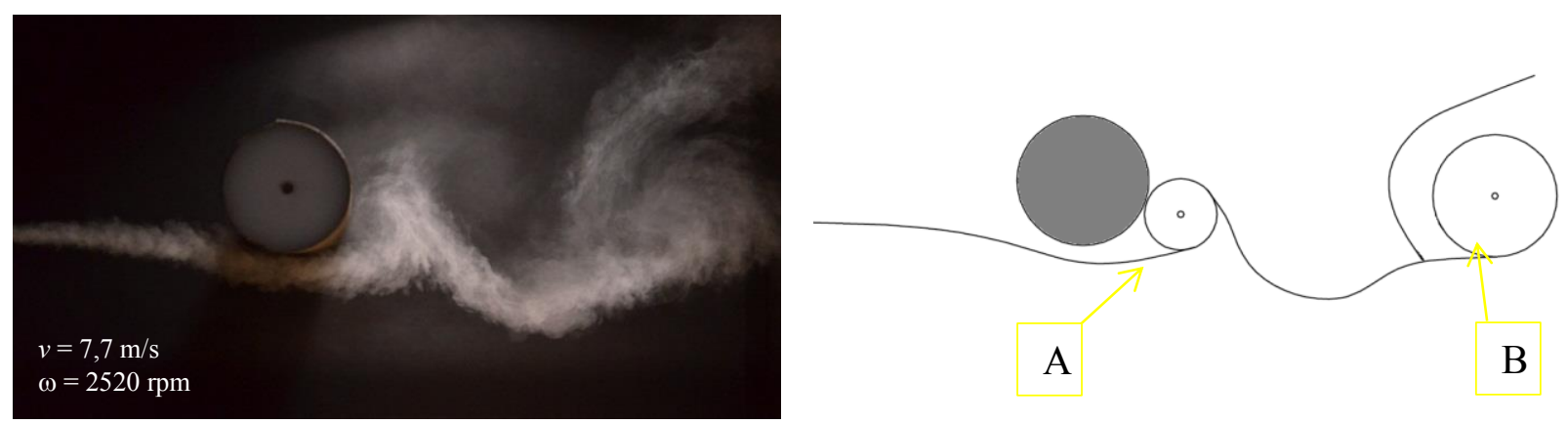

Figura 3.36 Configuración del flujo entorno al cuerpo.

Al igual que el caso anterior no se puede definir la fase de los torbellinos A y B de la figura 3.36. En este caso se observa al torbellino A adherido al cuerpo y torbellino $\mathrm{B}$ desplazado a aproximadamente 3 diámetros de distancia desde el centro del cuerpo. 
La figura 3.37 se corresponde el comportamiento $\mathrm{b}$ o f del flujo alrededor del cilindro circular con lija gruesa.
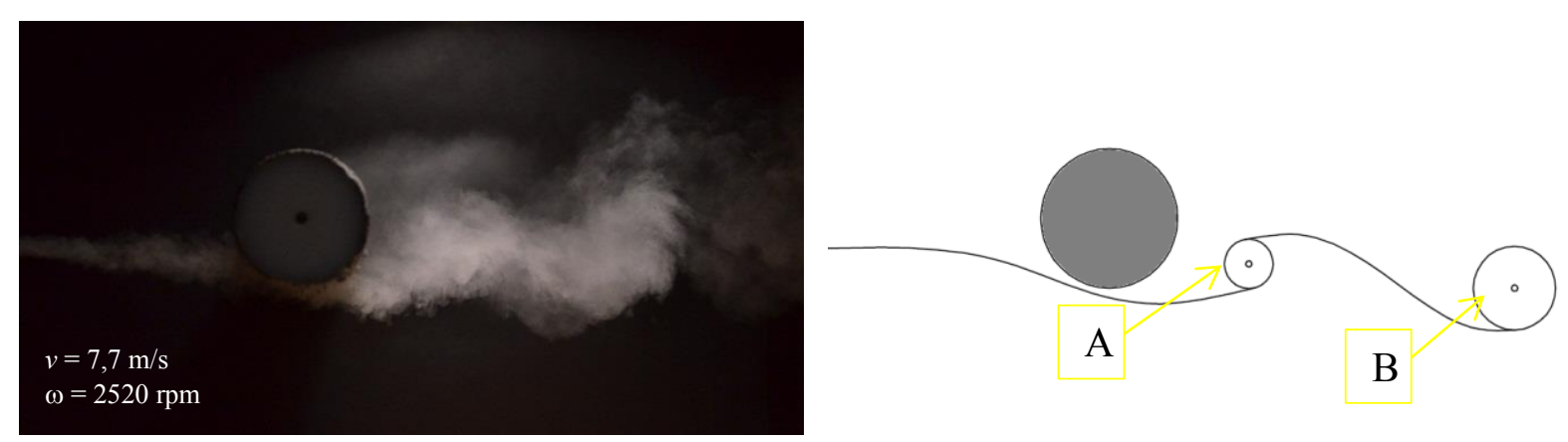

Figura 3.37 Configuración del flujo entorno al cuerpo.

Al igual que los casos anteriores no se puede definir la fase de los torbellinos A y B de la figura 3.37. En este caso se observa que el torbellino A está desplazado aproximadamente 1 diámetro de distancia desde el centro del cuerpo $\mathrm{y}$, el torbellino $\mathrm{B}$ está desplazado aproximadamente 3 diámetros.

La figura 3.38 se corresponde con el comportamiento a o f del flujo para el circular calado.
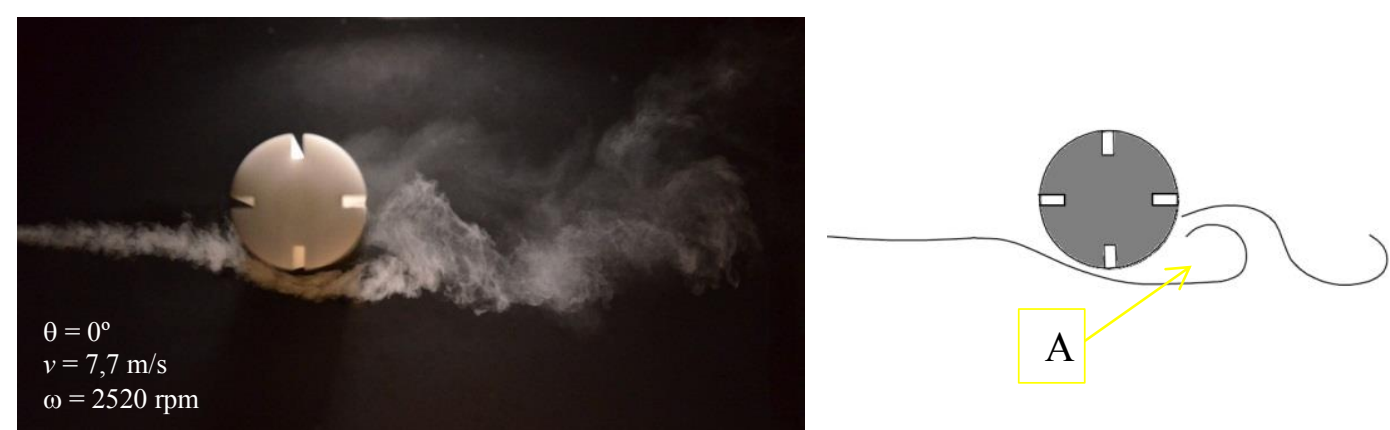

A
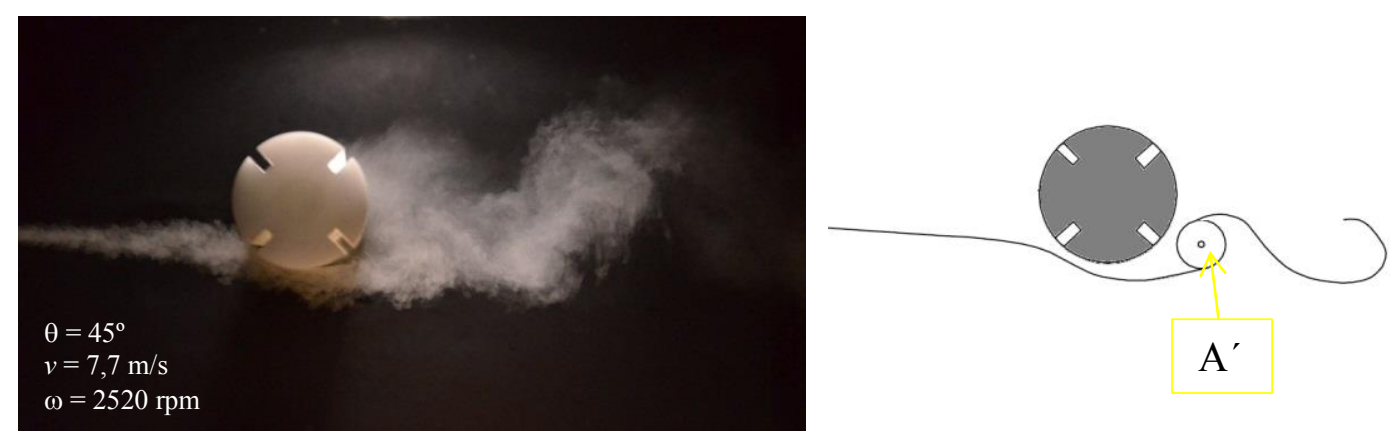

Figura 3.38 Configuración del flujo entorno al cuerpo para cada posición de $\theta$. 
$\theta=0^{\circ}$ : flujo desprendido formando un torbellino en A con sentido contrario a la corriente.

$\theta=45^{\circ}$ : torbellino $\mathrm{A}^{\prime}$ desprendiéndose del cuerpo.

\subsubsection{Conclusiones familia $\mathrm{V}$}

Cuando la relación $u / v<0.5$ no existe diferencia en la variación de $C_{L}$ para el CL, CLF y CLG (figura 3.33).

En los cilindros circulares sin patrones definidos no se pueden sincronizar las fotografías por lo que no son base para la discusión. En el caso del CC es diferente, pues se sincroniza el desprendimiento con el paso de las ranuras, aunque tampoco es tan claro como en el caso de los cuerpos con aristas.

El efecto de la lija fina (figura 3.36) genera mayor proximidad del torbellino a la pared del cuerpo que en el caso del circular liso. En el caso del cilindro recubierto con lija gruesa también se observa un patrón similar pero con menor proximidad del torbellino (figura 3.37). Estas diferencias en las proximidades del flujo pueden explicar el leve aumento de la sustentación de estos cuerpos respecto al circular liso.

Para el caso del cilindro calado (figura 3.38), se observa que el patrón de flujo depende de la posición de las ranuras. Puede observarse que el efecto del ranurado genera mayor proximidad del torbellino detrás del cuerpo, generando mucha mayor sustentación respecto a los otros, así mismo se observa que la tasa de cambio de $C_{L}$ es muy baja respecto a los otros lo que se puede atribuir a una variación en el número de Reynolds, ya que las ranuras generan cambios significativos en el patrón del flujo.

Así mismo, los comportamientos diferentes para el mismo cuerpo b y c (CLG) y, e y f (CC) pueden atribuirse al cambio de posición de los desprendimientos en el cuerpo producto de la rugosidad en el primer caso y de las caladuras en la superficie en el segundo caso. 


\subsection{COMPARACIÓN CON LA BIBLIOGRAFÍA}

En el presente apartado se comparan algunos de los casos estudiados con publicaciones en las que se estudian cuerpos similares. Si bien la geometría de los cuerpos y las condiciones de ensayo no son iguales a los de la presente Tesis, la comparación es útil para validar los ensayos de visualización y la representación gráfica de los patrones.

En la publicación "Wind Tunnel Measurement of the Surface Pressure Distribution on a Spinning Magnus Rotor" [7], se realiza el ensayo de un rotor tipo Savonius el cual gira en autorrotación y se mide la presión en la superficie del cuerpo. A su vez, se realizan visualizaciones para diferentes posiciones de giro que son las que se comparan con el cuerpo circular con aletas de la presente tesis.

En la figura 3.39 se puede observar la comparación del patrón de flujo de la mencionada publicación y la presente Tesis.

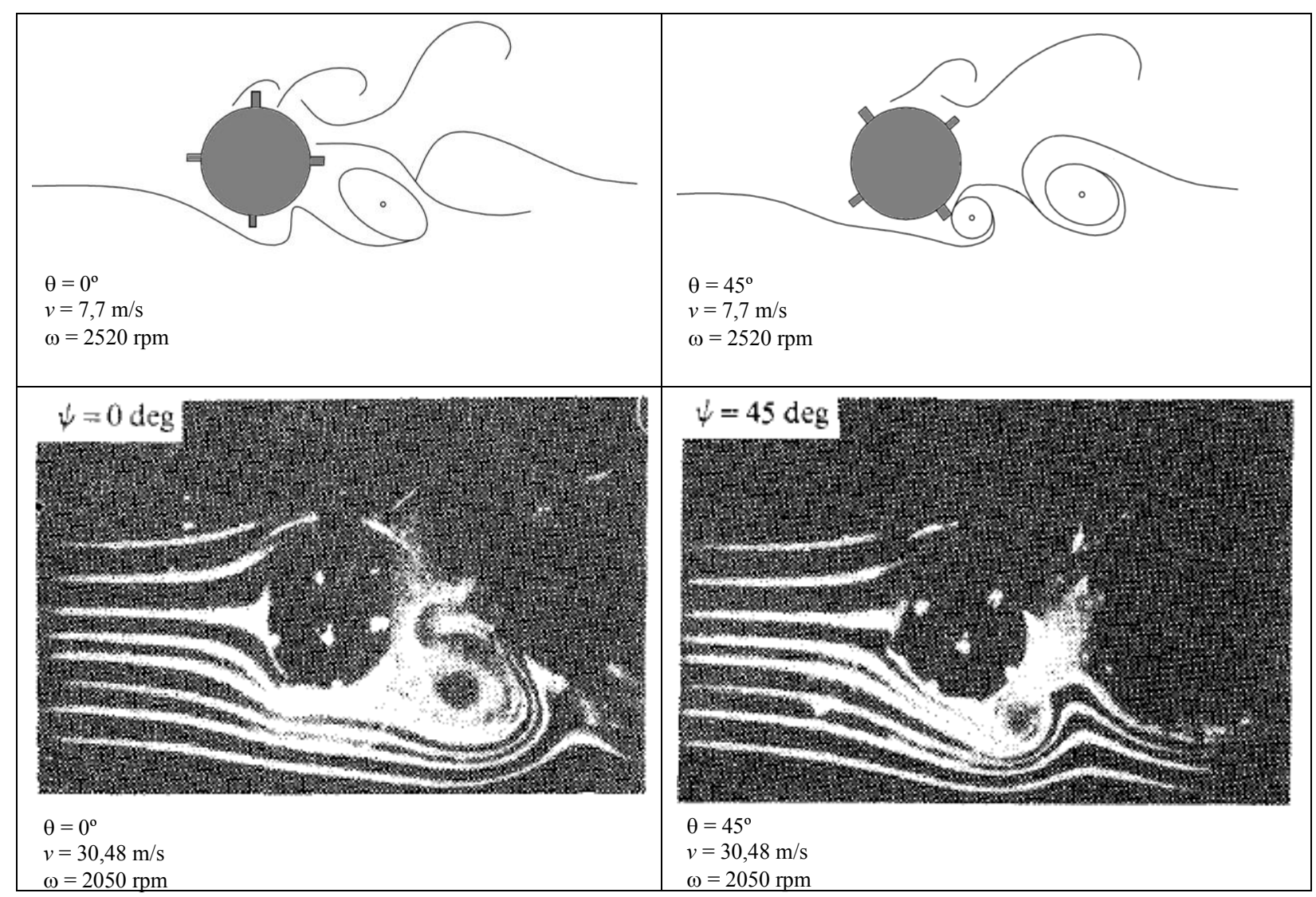

Figura 3.39 Comparación de la configuración del flujo entorno al cuerpo circular con aletas de la presente Tesis y el rotor de la publicación [7] para cada posición de $\theta$. 
Si bien las velocidades de rotación $\omega$ son similares para ambos casos, las velocidades de la corriente libre $v$ son muy diferentes, siendo mucho más alta en el caso de la publicación. Sin embargo el rotor Savonius posee un diámetro mucho mayor haciendo que la relación de velocidades $u / v$ sea de 0,46 en tanto que la del ensayo propio es de 0,6. Por tal son comparables los resultados en los que puede observarse un patrón muy similar con un torbellino de gran tamaño para $\theta=0^{\circ}$ y con un torbellino de menor tamaño en conjunto a la formación de otro detrás para el caso de $\theta=45^{\circ}$.

Por otro lado, en la publicación "Autorotation of Polygonal prisms with an upstream vane" [70], se realiza una introducción donde se describe el comportamiento del flujo en torno a una placa plana en autorrotación con una relación de velocidades $u / v$ de 0,45 , condición similar a la de la presente Tesis de 0,47 .

En la figura 3.40 se observa la comparación de los patrones de flujos de ambos casos.

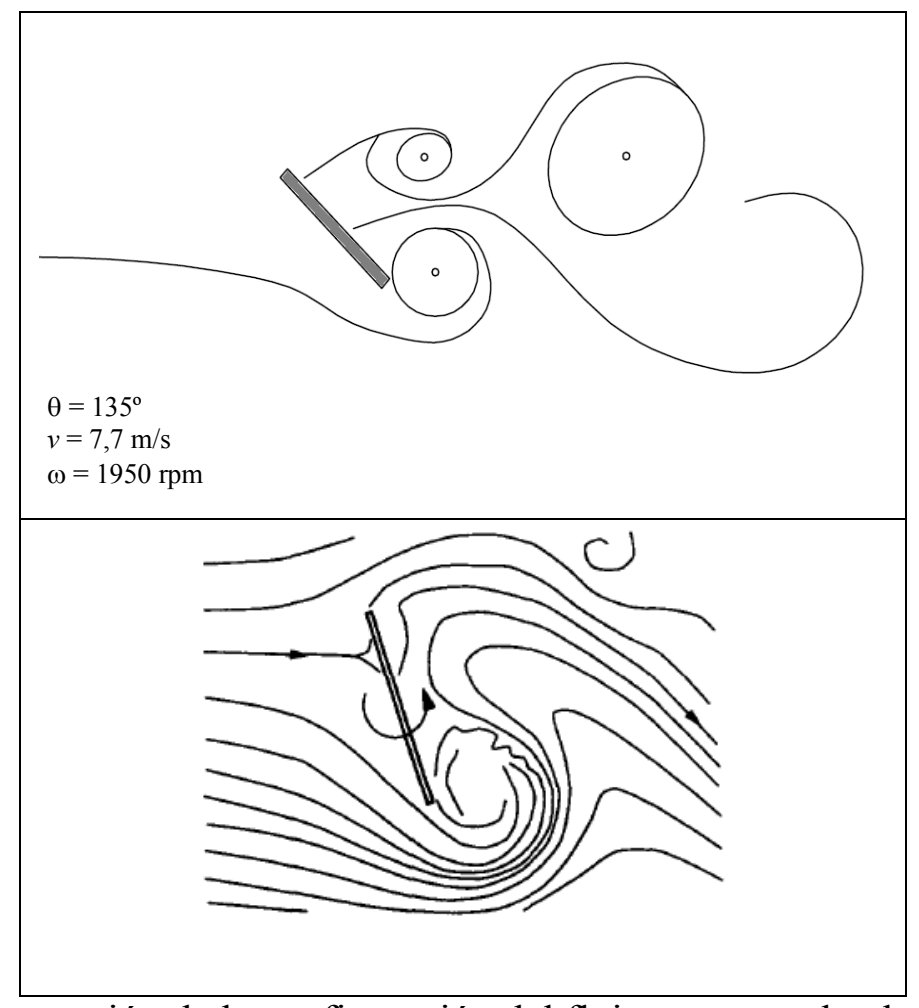

Figura 3.40 Comparación de la configuración del flujo entorno a la placa plana fina de la presente Tesis $\left(\theta=135^{\circ}\right)$ y la de la publicación [70] para una posición cercana. 
Puede observarse la generación de un torbellino mediano en la parte inferior y uno más pequeño en la parte superior en ambos casos. Si bien la geometría y la posición angular de las placas son diferentes, el patrón de flujo es similar.

\subsection{CONCLUSIONES}

A partir de lo expuesto en el apartado 3.2 se puede analizar la variación de las fuerzas de sustentación y resistencia en función de las velocidades de giro y de la corriente para los diferentes cuerpos.

El efecto Magnus que aparece sobre los cuerpos ensayados a la vista de las visualizaciones es una consecuencia de la generación de torbellinos, que es desigual entre las hileras superior e inferior de torbellinos que se desprenden del cuerpo.

La hilera inferior corresponde a una arista que avanza en la misma dirección de la corriente, por lo tanto con una velocidad relativa pequeña respecto al fluido, lo que da lugar a la generación de torbellinos bien definidos, intensos y, además, sincronizados con el paso de la arista.

La hilera superior corresponde a una arista que avanza en dirección contraria a la corriente, por lo tanto con una velocidad relativa al flujo elevada, y da lugar a torbellinos en general menos definidos (salvo en el caso de los triángulos y las placas planas) y de menor intensidad, como si no diera tiempo para que se produzca la generación completa del torbellino correspondiente.

En todas las familias puede observarse que el $C_{L}$ aumenta con el aumento de la relación de velocidades $u / v$, con diferentes pendientes entre las mismas.

A medida que disminuye la cantidad de caras se generan torbellinos de mayor tamaño. Esto explica la mayor sustentación en los triángulos respecto al resto de los cuerpos. Aunque las placas sólo poseen dos caras, el efecto turbulento producido por sus aristas filosas hace que no sean las que mayor fuerza de Magnus posean. 
Para las placas planas aparece claramente una calle de torbellinos similar a la de Von Kármán (es decir con una hilera superior y otra inferior de torbellinos alternados), lo que no se produce en todos los cuerpos estudiados.

Los torbellinos desprendidos en la hilera superior son en sentido horario y en la hilera inferior son en sentido anti horario.

Los distintos patrones en la estela de los cuerpos afectan más a la resistencia, ya que los torbellinos afectan más a la cara posterior del cuerpo que a las caras superior e inferior.

Por otro lado se observa que el hecho de poseer aristas redondeadas hace que los torbellinos se mantengan cercanos y detrás del cuerpo (estela más estrecha) durante más tiempo, razón por la cual se explican las diferencias en las curvas características de $C_{L}$ y $C_{D}$ respecto a los cuerpos con aristas afiladas. 


\section{CAPÍTULO 4}

\section{ANÁLISIS TEÓRICO}

\subsection{INTRODUCCIÓN}

El presente capítulo tiene como objetivo disponer de un modelo teórico que prediga el comportamiento aerodinámico de cuerpos similares a los ensayados al respecto del efecto Magnus.

Se estudian modelos publicados en la literatura y se desarrolla un modelo propio.

\subsection{APROXIMACIONES TEÓRICAS PUBLICADAS}

Se analizaron varias publicaciones en las que se presentan, desarrollos teórico-experimentales para determinar la fuerza de Magnus, en función de la velocidad de la corriente libre, la velocidad de rotación y los parámetros geométricos del cuerpo, con el objeto de aplicar los resultados de estos autores a los casos de la tesis y a la vez realizar un abordaje matemático.

En todos los casos estudiados se plantean ecuaciones para cuerpos cilíndricos circulares. Se realizó una comparación de los resultados de los ensayos de este cuerpo y la aplicación de la respectiva fórmula teórica, donde se obtuvieron las conclusiones siguientes.

La primera aproximación que se encuentra del análisis teórico es la de flujo potencial, planteado por L. Prandtl en 1926 [3] y, estudiada por Cuevas [10]. En la misma se determina el coeficiente de sustentación a partir de un análisis de la circulación alrededor de un cilindro circular:

Circulación:

$\Gamma=2 \pi R(\omega R)$ 
Sustentación (Kutta):

$L=\Gamma \rho v$

Por lo que el coeficiente de sustentación en función de las velocidades será:

$C_{L}=\frac{2 \pi \omega R}{v}=2 \pi\left(\frac{u}{v}\right)$

Este ajuste teórico se encuentra muy alejado de los resultados de los ensayos, tanto de la tesis como de cualquier otro publicado en la literatura. También el flujo considerado por Prandtl y el que se observa en los experimentos es muy diferente.

Por otro lado, Jacobson, Swanson, Iversen y Power [23] [26] [28] [29] [30] [32] realizan una gran variedad de estudios teóricos y analíticos relacionados con misiles en rotación, estudiando diferentes ángulos de ataque y generando ecuaciones matemáticas que determinan los coeficientes de sustentación producidos por el efecto Magnus.

Jacobson [23] realiza un extenso estudio para la aplicación en proyectiles y balística, de cuerpos en rotación, donde el efecto Magnus tiene una gran relevancia en la estabilidad del objeto.

Así mismo, Power [29] también ha realizado estudios sobre misiles en rotación donde trabaja con un análisis de la capa límite y los desprendimientos asimétricos como causales de la creación de un vórtice, generador de una fuerza lateral.

Sin embargo, todos estos análisis, están formulados para pequeños ángulos de ataque de la corriente respecto al eje de giro del cilindro, por lo que no aplica a los ensayos de la presente Tesis.

Cabe destacar que para el caso de una corriente perpendicular al eje de giro no se encontraron referencias de ecuaciones ni desarrollos que puedan determinar la fuerza de Magnus en función de la velocidad de rotación y la velocidad de la corriente libre. Por tal, se realiza un desarrollo propio donde se busca poder representar el mencionado efecto para la mayoría de los cuerpos ensayados en la presente Tesis. 


\subsection{DESARROLLO PROPIO}

Se realiza un desarrollo teórico para cuantificar el efecto Magnus en función de la formación de los torbellinos generados por los cuerpos. Como se observó en el capítulo 3, la generación de los mismos depende de la cantidad de aristas y de la relación de velocidades $u / v$.

Se parte de la definición del número de Strouhal, que determina la frecuencia del desprendimiento de torbellinos de los cuerpos:

$\mathrm{S}_{\mathrm{t}}=\frac{n_{d} \phi}{V_{r e l}}$

donde,

- $n_{d}$ : frecuencia de desprendimiento

- $\phi$ : diámetro del cuerpo

- $V_{\text {rel: }}$ velocidad relativa entre el cuerpo y la corriente incidente

Se considera el tiempo de desprendimiento de torbellinos como $t_{d}$ :

$t_{d}=\frac{1}{n_{d}}$

A partir de las ecuaciones (12) y (13) se obtiene:

$t_{d}=\frac{1}{\mathrm{~S}_{\mathrm{t}}} \frac{\phi}{V_{\text {rel }}}$

Por otra parte, si el cuerpo posee aristas, el tiempo de paso de la arista limita el tiempo disponible para generar un torbellino. Por tal se define $t_{c}$ como el tiempo transcurrido entre pasos de aristas sucesivas por el punto con condición de contorno (con giro) como:

$t_{c}=\frac{1}{f_{g} n_{l}}$

donde $f_{g}$ es la frecuencia de giro, y $n_{l}$ es la cantidad de caras del cuerpo. 
Entonces, se propone que para que se genere un torbellino, para el cuerpo en rotación, debe ocurrir que $t_{c}>t_{d}$, puesto que si $t_{c}$ es pequeño (en relación a $t_{d}$ ) el cuerpo se comporta como "sin rotación" y prima el efecto de desprendimientos de un cuerpo estático por sobre uno en rotación. Se cumple, entonces,

$$
\frac{1}{f_{g} n_{l}}>\frac{\phi}{\mathrm{S}_{\mathrm{t}} V_{r e l}}
$$

Por otra parte, para un cuerpo romo, la velocidad local en el punto de máxima velocidad es aproximadamente el doble que la velocidad de la corriente libre por efecto de la desviación de flujo (figura 4.1).

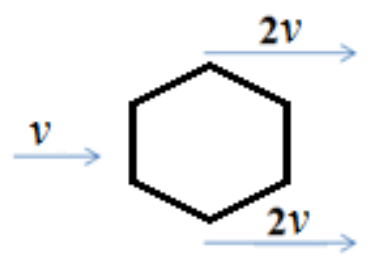

Figura 4.1 Velocidades en torno al cuerpo.

A su vez, si el cuerpo posee rotación, las velocidades en torno al cuerpo se muestran en la figura 4.2 .

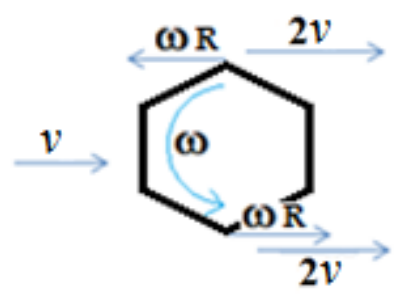

Figura 4.2 Velocidades en torno al cuerpo en rotación.

Por tanto la velocidad relativa en la capa inferior está dada por,

$$
V_{r e l}=2 v-u=v\left(2-\frac{u}{v}\right)
$$


Reemplazando en la ecuación (16),

$$
\frac{\mathrm{S}_{\mathrm{t}}\left(2-\frac{u}{v}\right)}{n_{l} \frac{u}{v} \frac{1}{\pi}}>1
$$

Considerando un $\mathrm{S}_{\mathrm{t}}=0,1$ aproximado para cuerpos con bordes afilados:

$$
\frac{0,3\left(2-\frac{u}{v}\right)}{n_{l} \frac{u}{v}}>1
$$

Despejando,

$$
\frac{2}{\frac{n_{l}}{0,3}+1}>\frac{u}{v}
$$

La ecuación (20) muestra matemáticamente que a menor cantidad de caras $\left(n_{l}\right)$ el límite en $u / v$ es más grande. Por lo tanto, a menor número de caras la formación de torbellinos se produce con mayor facilidad, pues el límite de $u / v$ es mayor. Esto no se puede aplicar para los cuerpos circulares $\left(n_{l}=\infty\right)$, ya que no tienen aristas en las que se pueda considerar el arranque de la generación de un torbellino y por lo tanto definir el tiempo $t_{c}$. Por lo tanto, para que se generen los desprendimientos en las aristas debe cumplirse la desigualdad (20).

A partir de esta conclusión inicial continúa el desarrollo: para analizar la generación de sustentación de un cuerpo en rotación en función de la cantidad de caras que posea el mismo se consideran tres tiempos diferentes.

Además del tiempo de desprendimiento de torbellinos $t_{d}$ del cuerpo sin rotación y el tiempo de desprendimiento de torbellinos $t_{c}$ del cuerpo rotando, definidos en las ecuaciones (14) y (15), se define el tiempo de residencia $t_{r}$, como el tiempo que tarda una partícula en recorrer una distancia igual al diámetro del cuerpo:

$$
t_{r}=\frac{\phi}{v}
$$


Entonces, se define el tiempo de residencia considerando un lado $t_{r l}$, como el tiempo que tarda una partícula para recorrer una distancia igual a un lado del cuerpo:

$$
t_{r_{l}}=\frac{l}{v}=\frac{\frac{\text { Perimetro }}{n_{l}}}{v} \cong \frac{\pi \phi}{n_{l} v}=\frac{\pi}{n_{l}} t_{r}
$$

Se puede concluir que para valores de $n_{l}$ grandes (cuerpos de 10 caras):

$$
t_{r_{l}} \cong \frac{1}{3} t_{r}
$$

A modo de ejemplo, considerando valores típicos de los ensayos experimentales:

- $\phi=0,036 \mathrm{~m}$ (promedio de los diámetros de todos los cuerpos)

- $v=10 \mathrm{~m} / \mathrm{s}$

- $\omega=2520 \mathrm{rpm}=42 \mathrm{rps}$

- $\mathrm{S}_{\mathrm{t}}=0,2$ (cuerpos romos)

- $\mathrm{S}_{\mathrm{t}}=0,1$ (cuerpos con aristas)

Reemplazando estos valores en las ecuaciones (14), (15) y (21) se obtienen los siguientes resultados:

$t_{d}=1,8 \times 10^{-2} \mathrm{~s} \quad$ (considerando el diámetro)

$t_{d}=3,6 \times 10^{-2} \mathrm{~s} \quad$ (considerando un lado)

$t_{c}=\frac{2,4 \times 10^{-2}}{n_{l}} \mathrm{~s}$

$t_{r}=3,6 \times 10^{-3} \mathrm{~s}$

Se observa que:

Si el cuerpo no gira, el tiempo necesario para generar un torbellino en cualquiera de las dos capas (superior o inferior) es $t_{d}=1,8 \times 10^{-2} \mathrm{~s}$ 
Si el cuerpo gira, cambian las condiciones de contorno en un tiempo $t_{c}=\frac{2,4 \times 10^{-2}}{n_{l}} \mathrm{~s}$, es decir, del mismo orden que el que tardaría en formarse un torbellino si el cuerpo no girase.

El tiempo de residencia de una partícula fluida es $t_{r}=3,6 \times 10^{-3} \mathrm{~s}$, o menor si se considera sólo el paso por un lado del cuerpo. Esto quiere decir que como $t_{r}<<t_{c}$ el proceso puede considerarse como cuasi estacionario (es como si estuviera siempre en esa configuración, con las velocidades del caso no estacionario). De manera que el desprendimiento de la corriente en la arista y por lo tanto la generación del torbellino correspondiente está condicionada por la posición de las aristas, y la velocidad del giro. Por lo tanto, no se desprende una calle de Von Kármán modulada por la posición de las aristas en el movimiento de giro (lo que ocurriría si la velocidad de giro fuese muy lenta).

En la arista superior, que avanza contra el fluido, el tiempo de residencia es menor (se considera que $V_{r e l}$ es del orden de $2 v+u$ ), el tiempo disponible para que se genere el torbellino se reduce, y no se llegan a formar torbellinos significativos.

En la arista inferior, en cambio, el movimiento de la arista con respecto al fluido es más lento (se considera que $V_{r e l}$ es del orden de $2 v-u$ ) por lo que aumenta el tiempo disponible para la formación de torbellinos, y de hecho se observan torbellinos de tamaño grande.

El tiempo de residencia en un lado (inferior) es:

$t_{r_{i i}}=\frac{l}{V_{r e l}}=\frac{\pi \phi}{n_{l}(2 v-u)}=t_{r} \frac{\pi}{n_{l}} \frac{v}{(2 v-u)}=t_{r} \frac{\pi}{n_{l}} \frac{1}{\left(2-\frac{u}{v}\right)}$

Por lo tanto, la relación entre el tiempo de residencia superior e inferior es:

$$
\frac{t_{r_{l_{s}}}}{t_{r_{l_{i}}}}=\frac{\left(2-\frac{u}{v}\right)}{\left(2+\frac{u}{v}\right)}
$$

Y para el rango de $u / v$ de los ensayos $(0,3<u / v<0,9)$, los valores de la relación (25) se encuentran aproximadamente entre 0,7 y 0,4 . Por lo tanto, los torbellinos tienen más tiempo para desarrollarse en la arista inferior que en la superior. 
Por ejemplo, para un mismo valor de la relación $u / v$, el tiempo de residencia para un triángulo es mayor que para un hexágono, entonces, el torbellino presente en la cara inferior para el triángulo es más grande (más desarrollado) que para el hexágono. Esto se observa en los ensayos donde, además, el coeficiente de sustentación $C_{L}$ para el triángulo (torbellinos más grandes) es mayor que para el hexágono.

Como una primera conclusión se puede decir que la sustentación aparece por un aumento de la intensidad de torbellinos desprendidos en la arista inferior (en el paso). El efecto de los torbellinos desprendidos en la arista superior es pequeño, lo mismo que la intensidad de dichos torbellinos.

Para determinar el comportamiento del $C_{L}$ en función del número de caras de los cuerpos y la relación de velocidades $u / v$, se plantea que la sustentación está relacionada con la interacción de los torbellinos desprendidos con el cuerpo que gira (es sabido que la vorticidad total desprendida desde la vecindad del cuerpo es igual y opuesta a la circulación sobre el mismo cuerpo), y por lo tanto, depende de la circulación generada en la unidad de tiempo, que es proporcional a la presión dinámica y es debida a la descompensación de la estela (entre la capa superior y la capa inferior de torbellinos) respecto a la misma cuando el cuerpo no gira $(\omega=0)$.

Se plantea entonces, que la circulación total es:

$$
\Gamma_{\text {total }}=n_{t} \Gamma_{1}
$$

donde:

$n_{t}$ : número de torbellinos en la unidad de tiempo; $n_{t}=n_{l} f_{g}:$ números de pasos de la arista por la posición inferior (se observó que los torbellinos desprendidos en la cara inferior del cuerpo son mucho más intensos que los de la arista superior, por lo que se considera que predominan sobre estos en cuanto a la influencia en la sustentación sobre el cuerpo).

$\Gamma_{1}$ : intensidad de un torbellino, proporcional a la presión dinámica y al tiempo que tiene para crecer antes de ser arrojado por el paso de la arista siguiente, y se puede expresar como:

$\frac{\Gamma_{1}}{q} \cong k\left(t_{r}+t_{c}-\alpha t_{d}\right)$ 
donde $k$ es una constante de proporcionalidad con las dimensiones adecuadas.

La ecuación (27) permite definir dos comportamientos:

Comportamiento 1: para cuerpos con muchas caras ( $n_{l}$ igual o mayor a 8 caras), donde se generan muchos torbellinos pequeños. Si el cuerpo tiene muchas caras tendiendo al cuerpo circular, el tiempo que tiene un torbellino para crecer antes de ser lanzado por la corriente es proporcional al tiempo de residencia. En este caso se obtiene que:

$\Gamma_{1}=q k t_{r}$ : corresponde al caso en el que el torbellino que se genera tiene el tiempo "justo", por lo tanto, $t_{c}=\alpha t_{d}$, es decir, el tiempo de paso de la arista $t_{c}$ es el adecuado para que se genere un torbellino que necesita un tiempo $\alpha t_{d}$. El parámetro $\alpha$ de proporcionalidad se introduce para simbolizar $t_{c} \cong t_{d}$.

Comportamiento 2: para cuerpos con pocas caras $\left(3<n_{l}<8\right.$ caras) el torbellino tiene más tiempo para desarrollarse, y se considera que la diferencia entre los tiempos $t_{c}$ y $t_{d}$ influye en la sustentación, considerando si los torbellinos que se desarrollan se mantienen cercanos o alejados del cuerpo, y generan cambios en la misma. Al tener pocas caras, el tiempo que tiene el torbellino para crecer y ser lanzado por la corriente está dado por $t_{c}$.

Comportamiento 3: se define el comportamiento 3 para cuerpos con $n_{l}<3$ caras, que no se encuentra incluida en el desarrollo del presente algoritmo.

En la figura 4.3 se representa la variación de la pendiente de la curva característica de cada cuerpo en función de la cantidad de caras, $n_{l}$. Se han marcado los tres comportamientos antes 
mencionados.

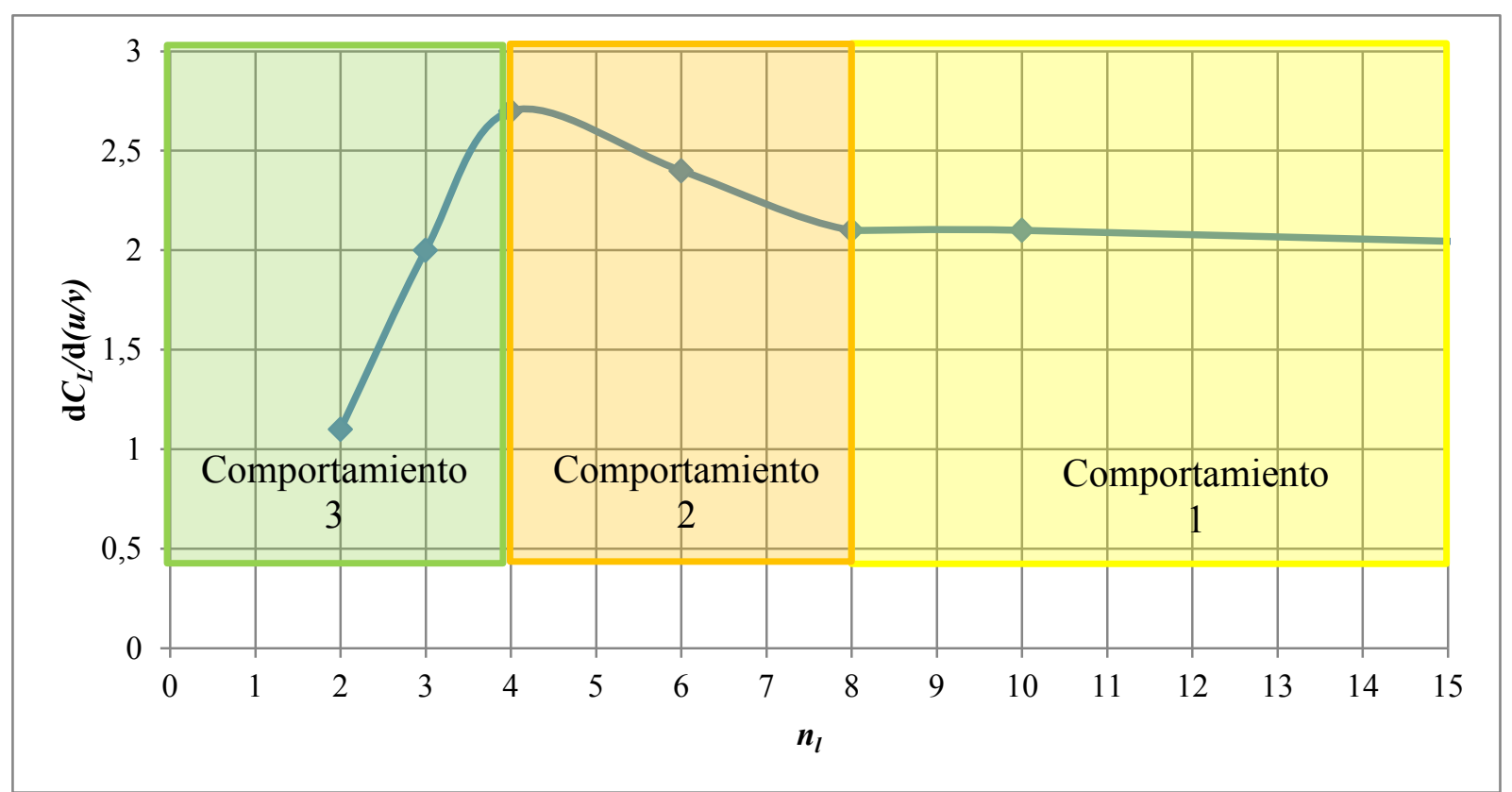

Figura 4.3 Pendiente de la curva característica de $C_{L}(u / v)$ en función de la cantidad de caras de los cuerpos. Resultados experimentales.

Por lo tanto se desarrolla la ecuación (28),

$\frac{\Gamma_{\text {total }}}{q k}=n_{l} f_{g}\left(t_{r}+\frac{1}{f_{g} n_{l}}-\alpha \frac{1}{\mathrm{~S}_{\mathrm{t}}} \frac{\phi}{U_{\infty}}\right)$

Desarrollando,

$\frac{\Gamma_{\text {total }}}{q k}=n_{l} f_{g} t_{r}+1-\alpha \frac{n_{l} f_{g}}{\mathrm{~S}_{\mathrm{t}}} \frac{\phi}{U_{\infty}}$

Que es válida para $t_{c}>\alpha t_{d}$

En tanto que $k t_{r}$ será dependiente de la velocidad del flujo, $v$, y del tamaño del lado $l$.

Entonces, considerando $\beta$ como una constante de proporcionalidad,

$t_{r}=\frac{\beta}{n_{l}} \frac{\phi}{v}$ 
Por lo tanto, la circulación total queda expresada como:

$\frac{\Gamma_{\text {total }}}{q k}=\beta n_{l} \frac{\omega}{2 \pi} \frac{\phi}{v n_{l}}+1-\frac{\alpha n_{l} \omega \phi}{2 \pi \mathrm{S}_{\mathrm{t}}(2 v-u)}$

Dejando en función de la relación $u / v$ :

$C_{L} \cong \frac{\Gamma_{\text {total }}}{q}=(k \beta) \frac{1}{\pi} \frac{u}{v}+k-(k \alpha) \frac{n_{l}}{\pi S_{t}} \frac{\left(\frac{u}{v}\right)}{\left(2-\frac{u}{v}\right)}$

Se procedió a determinar los coeficientes de proporcionalidad $k, \beta$ y $\alpha$ que mejor ajusten con valores únicos a la curvas características de cuerpos de 4 a 10 caras, a partir de ajustar la ecuación (32) con las curvas características de $C_{L}(u / v)$ para los cuerpos con 4, 6 y 8 caras (para los cuerpos con 10 caras la pendiente de $C_{L}(u / v)$ es la misma que para los de 8).

En las figuras 4.4, 4.5 y 4.6 se muestran los ajustes para estos cuerpos:

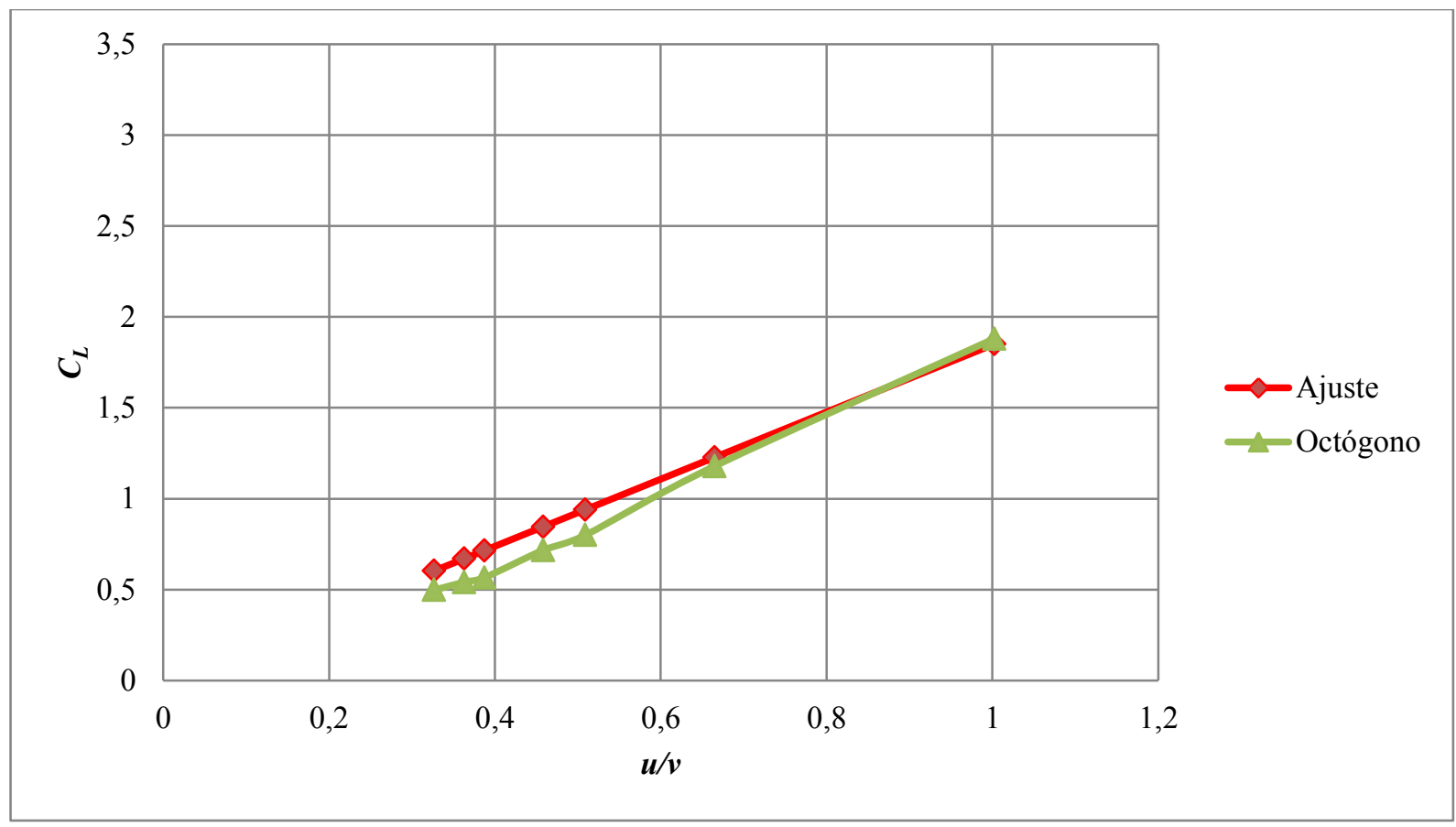

Figura 4.4 Curva característica de $C_{L}(u / v)$ (experimental) y ajuste de la ecuación (32) para el octógono. 


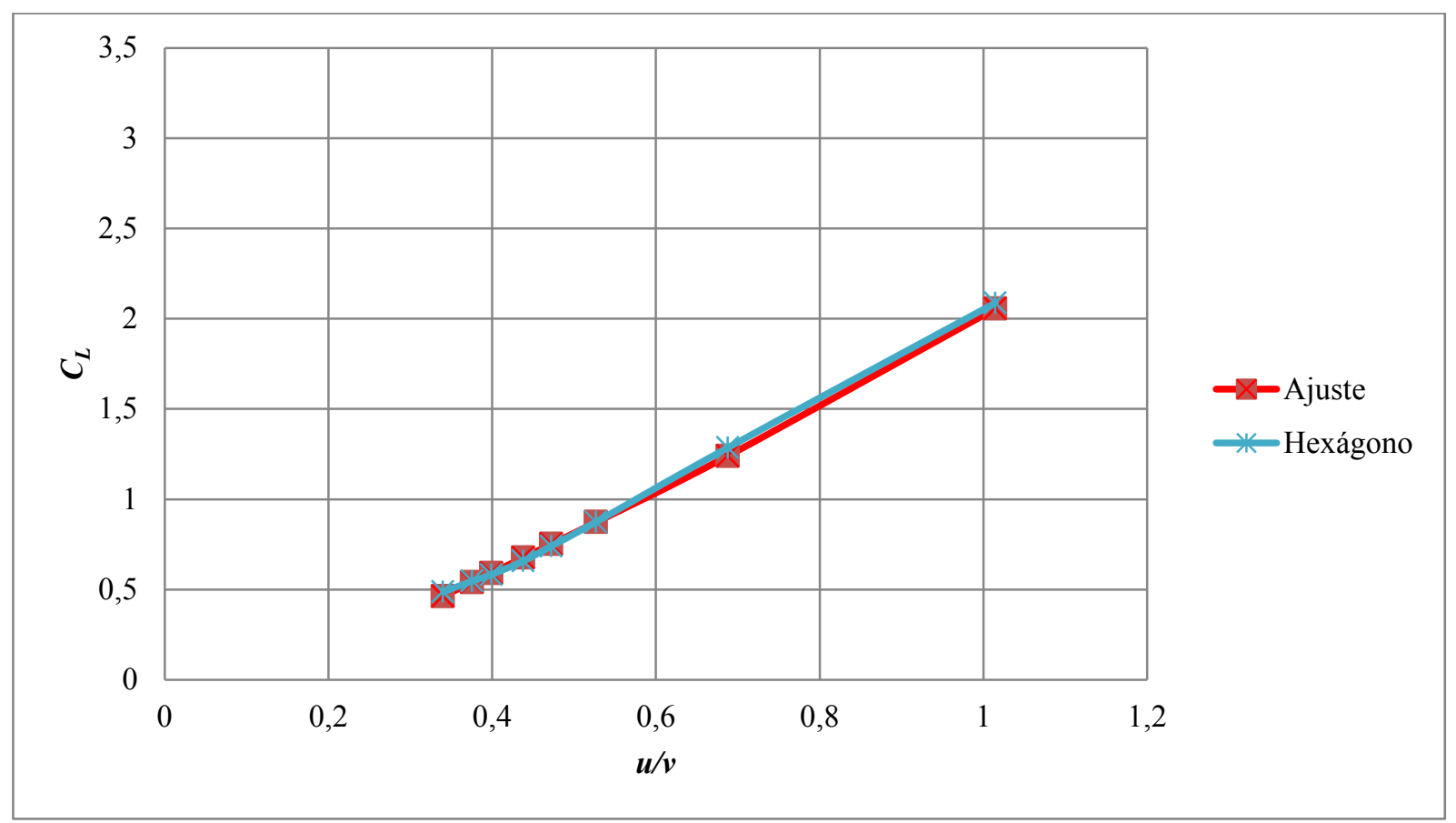

Figura 4.5 Curva característica de $C_{L}(u / v)$ (experimental) y ajuste de la ecuación (32) para el hexágono.

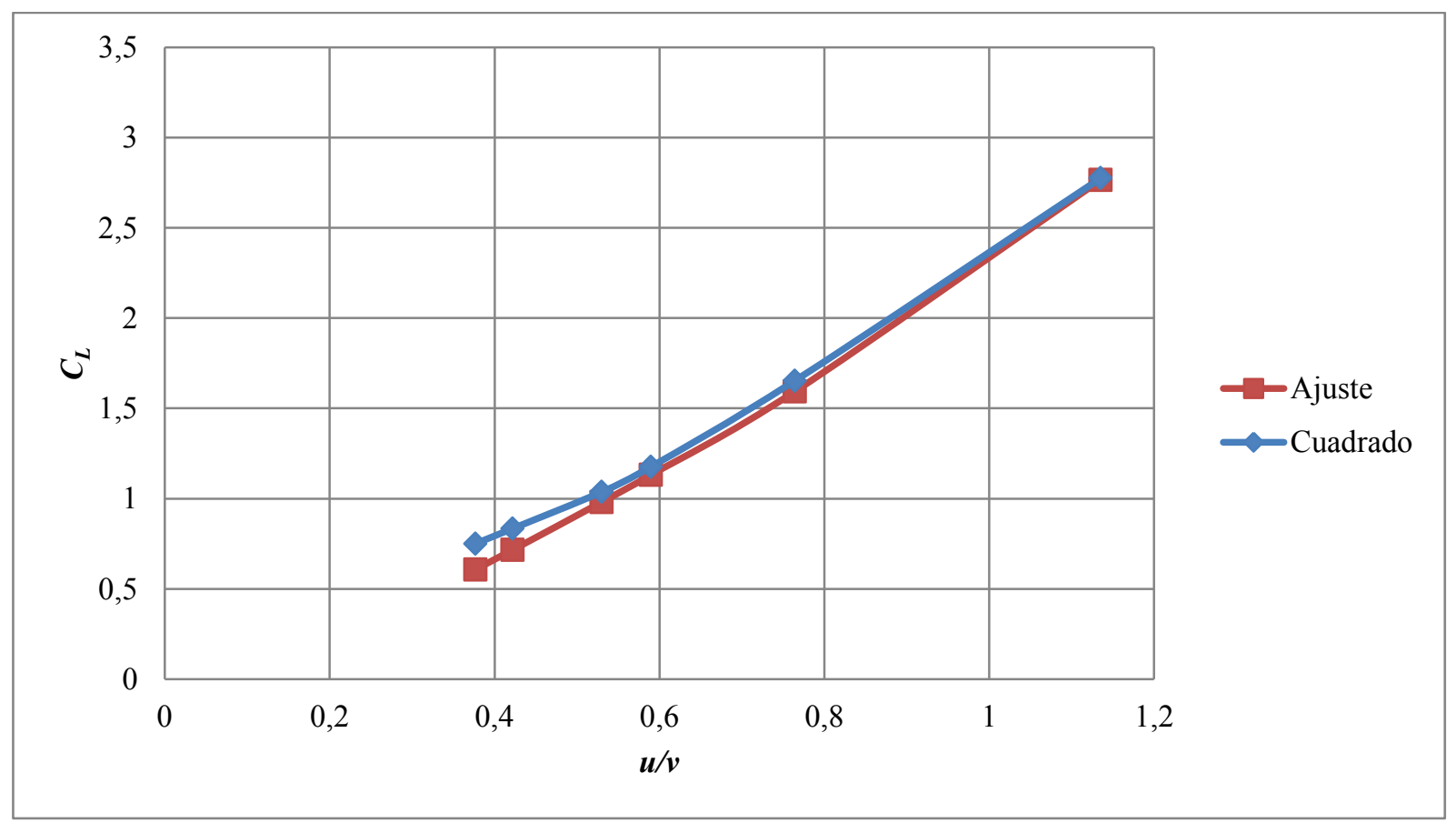

Figura 4.6 Curva característica de $C_{L}(u / v)$ (experimental) y ajuste de la ecuación (32) para el cuadrado. 
De esta manera se obtuvieron los valores que se presentan en la tabla 4.1.

Tabla 4.1. Parametros de proporcionalidad de la ecuación (32) para el rango $3<n_{l}<8$ caras.

\begin{tabular}{|c|c|}
\hline Parámetro & Valor \\
\hline$k \beta$ & 5,8 \\
\hline$k$ & $-0,25$ \\
\hline$k \alpha$ & $-0,055$ \\
\hline
\end{tabular}

Se observa que cuando $t_{c} \cong \alpha t_{g}$ la circulación total crece linealmente con $u / v\left(n_{l}\right.$ mayor o igual a 8 caras).

Por otro lado, estos parámetros permiten definir la variación del $C_{L}$ con la variación de la relación $u / v$ para los cuerpos ensayados de 4 y 6 caras.

Los valores negativos de $k$ y $k \alpha$ permiten concluir que la elección inicial del signo de $t_{c}-\alpha t_{d}$ no ha sido correcta y que el tiempo de generación de un torbellino es mayor al tiempo de desprendimiento del mismo $\left(t_{c}>t_{d}\right)$, lo que sugiere que sigue creciendo mientras se mantiene adherido al cuerpo por más tiempo y por tal genera mayor sustentación (si se dispone de menos aristas, mayor tiempo de crecimiento).

En cuanto al comportamiento 3 para los cuerpos con $n_{l}$ menor a 4 caras no se ha encontrado una ecuación representativa, ya que a la formación de torbellinos no se le puede asignar tiempos de paso y generación, debido a que el comportamiento fluidodinámico es complejo por la poca cantidad de caras y la turbulencia asociada a las mismas.

El resultado del ajuste aplicado a la pendiente de la curva característica de los ensayos (figura 4.3) se puede observar en la figura 4.7. 


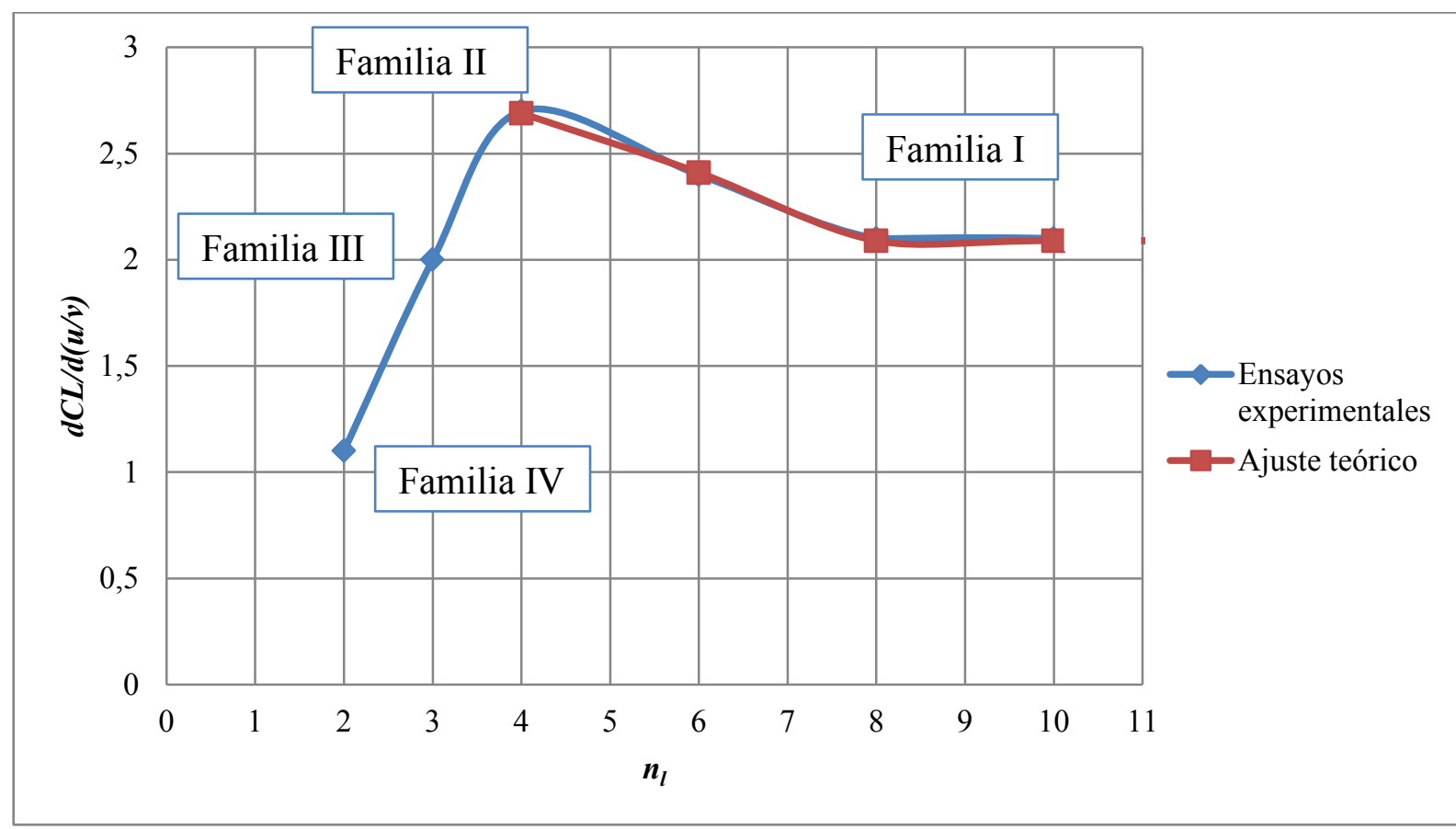

Figura 4.7 Pendiente de la curva característica de $C_{L}(u / v)$ en función de la cantidad de caras de los cuerpos y ajuste teórico.

Se observa en la figura 4.7 que existen tres regímenes donde se puede determinar lo siguiente:

$n_{l} \geq 8$ caras: el aumento de número de lados genera más torbellinos, pero más pequeños, de forma que se compensa el efecto de la cantidad con el tamaño de los mismos.

$4 \leq n_{l} \leq 8$ caras: al disminuir el número de lados (hasta 4) los torbellinos se encuentran más tiempo adheridos al cuerpo y por tal generan mayor sustentación. Este régimen está asociado a la condición 2.

$n_{l} \leq 4$ : Caso singular. No desarrollado teóricamente. 


\subsection{CONCLUSIONES}

Las aproximaciones teóricas publicadas por diversos autores no permiten ajustar los resultados de manera teórica, porque consideran pequeños ángulos de incidencia $\left(\alpha<20^{\circ}\right)$ de la corriente sobre los cuerpos en rotación. Así mismo, las aproximaciones son para cuerpos cilíndricos circulares que no contemplan cuerpos con caras.

Por otro lado se analizaron los casos de ensayo con flujo potencial, muy utilizado en la bibliografía para explicar el efecto Magnus, pero para casos donde los cuerpos poseen aristas no puede utilizarse, ya que no contempla el efecto de los torbellinos generados por las mismas.

Para contemplar los cuerpos con caras se realizó un planteo teórico a partir de considerar tiempos asociados al desarrollo de los torbellinos en el cuerpo. El mismo permite definir, a partir de la cantidad de caras y la relación de velocidades $u / v$, el coeficiente de sustentación $C_{L}$ en los cuerpos. Dicho algoritmo se encuentra asociado a los parámetros $k, \alpha$ y $\beta$, que son propios de la presente Tesis. El mismo se encuentra validado para cuerpos que posean 4 o más cantidad de caras.

Es de gran importancia haber podido definir el mencionado algoritmo ya que puede considerarse como un aporte novedoso a la bibliografía referida a la temática del efecto Magnus. 


\section{CAPITULO 5}

\section{ESTUDIO DE TRAYECTORIAS}

\subsection{INTRODUCCIÓN}

A modo de introducción se puede mencionar que Holmes [48] [49], Baker [50] y Tachikawa [71] han realizado diversos estudios de trayectorias de placas planas en vuelo libre. Han realizado ensayos experimentales en túnel de viento y han desarrollado ecuaciones para predecir dicho movimiento. Así mismo, Visscher [51] y Kakimpa [53] [54] [55], ha realizado estudios experimentales para determinar trayectorias de chapas de techos que se vuelan por acción del viento.

Cabe destacar que no se ha encontrado en la bibliografía estudios de trayectorias del vuelo de cuerpos llevados por el viento que no sean placas planas.

A partir de las caracterizaciones de los cuerpos efectuadas en el Capítulo 3 se realiza un estudio de las posibles trayectorias de aquellos cuerpos que poseen autorrotación. Tomando como referencia las publicaciones "The Debris Flight Equations" [50] y "Autorotation of many-sided bodies in an airstream" [18], y utilizando el Software Matlab se desarrolló un cálculo que permite estudiar el vuelo libre de los cuerpos y compararlos entre sí.

\subsection{DESARROLLO}

El presente estudio considera un cuerpo en reposo el cual es sometido a la acción del viento, y a partir de este comienza un desplazamiento con autorrotación. La autorrotación hace que el cuerpo se vea afectado por el efecto Magnus y por tal, se genere una fuerza perpendicular a la velocidad del viento, que hace que el cuerpo al desplazarse recorra mayor distancia que cuando no posee un giro. 


\subsubsection{Cuerpos con autorrotación}

A partir de los ensayos experimentales de Skews [18] se puede determinar que los cuerpos que poseen autorrotación son las placas planas, los triángulos, los cuadrados y el hexágono. En la mencionada publicación se define la relación de velocidades $u / v$ de autorrotación que es única para cada cuerpo en las condiciones estudiadas por dicho autor. Los mismos se presentan en la tabla 5.1.

Tabla 5.1 Relación de velocidades $u / v$ en función de los cuerpos según [18].

\begin{tabular}{|c|c|}
\hline Cuerpo & $\boldsymbol{u} / \boldsymbol{v}$ \\
\hline Placa plana fina & 0,43 \\
\hline Triángulo & 0,50 \\
\hline Cuadrado & 0,49 \\
\hline Hexágono & 0,35 \\
\hline
\end{tabular}

Para el presente desarrollo no se consideran los cuerpos con aristas redondeadas ni el circular con aletas, en tanto que de las placas planas sólo se considera la de espesor fino. El resto de los cuerpos ensayados para la presente tesis no presentan autorrotación.

\subsubsection{Ecuaciones de movimiento}

En la publicación [50], Baker presenta un análisis de las ecuaciones que forman el modelo matemático correspondiente al vuelo de un objeto en rotación. Realiza un estudio de las variables que intervienen en dicho movimiento considerando al mismo en dos dimensiones. En la figura 5.1 se presenta el esquema de la configuración estudiada con la definición de la rotación empleada, y a continuación un desarrollo de las ecuaciones de movimiento.

Se realiza un análisis en dos dimensiones; el eje de rotación de los cuerpos es perpendicular al plano $x y$. 

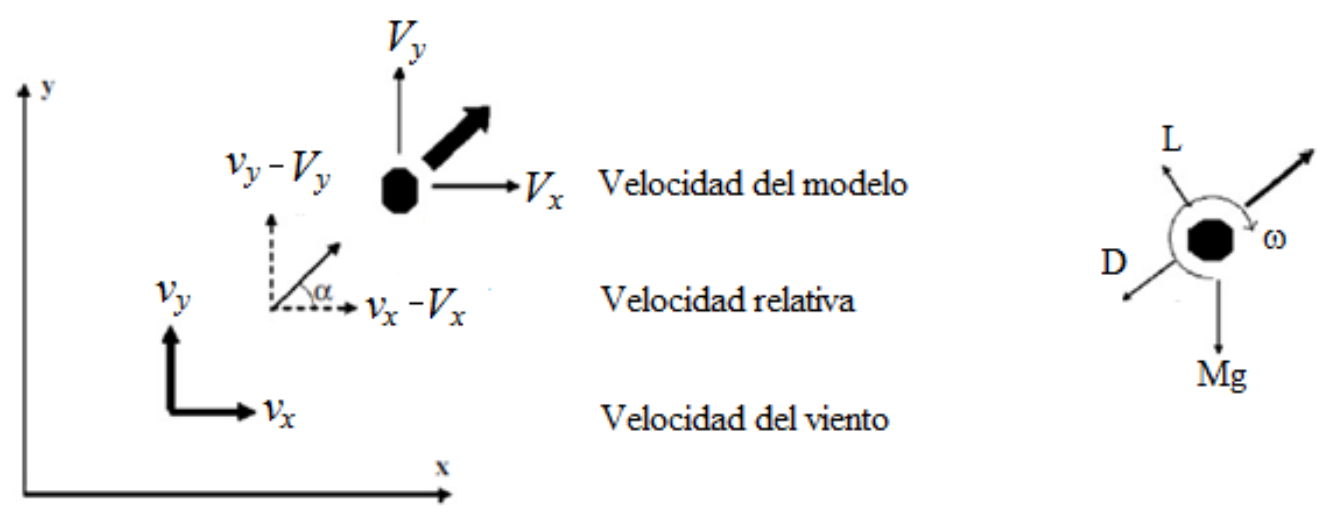

Figura 5.1 Configuración estudiada.

$$
\begin{aligned}
& M \frac{\mathrm{d}^{2} x}{\mathrm{~d} t^{2}}=M \frac{\mathrm{d} V_{x}}{\mathrm{~d} t}=\left(\frac{S \rho}{2}\right)\left(C_{D} \cos \alpha-\left(C_{L}+C_{L A}\right) \operatorname{sen} \alpha\right)\left(\left(v_{x}-V_{x}\right)^{2}+\left(v_{y}-V_{y}\right)^{2}\right), \\
& M \frac{\mathrm{d}^{2} y}{\mathrm{~d} t^{2}}=M \frac{\mathrm{d} V_{y}}{\mathrm{~d} t}=\left(\frac{S \rho}{2}\right)\left(C_{D} \operatorname{sen} \alpha+\left(C_{L}+C_{L A}\right) \cos \alpha\right)\left(\left(v_{x}-V_{x}\right)^{2}+\left(v_{y}-V_{y}\right)^{2}\right)-M g, \\
& I \frac{\mathrm{d}^{2} \theta}{\mathrm{d} t^{2}}=I \frac{\mathrm{d} \omega}{\mathrm{d} t}=\left(\frac{\left(C_{M}+C_{M A}\right) S l \rho}{2}\right)\left(\left(v_{x}-V_{x}\right)^{2}+\left(v_{y}-V_{y}\right)^{2}\right) .
\end{aligned}
$$

Considerando que $\alpha$ es el ángulo definido entre la trayectoria del cuerpo y la velocidad relativa, se pueden ajustar las funciones trigonométricas y reemplazar por la relación de velocidades. Por otra parte se considera que los cuerpos sólo tienen sustentación por efecto de la rotación, por lo que interviene solamente el coeficiente $C_{L A}$ (ver 5.3. Conclusiones), de esta manera se arriba a las ecuaciones (36), (37) y (38), que son las que se van a utilizar para el presente estudio.

$$
\begin{aligned}
& \frac{\mathrm{d}^{2} x}{\mathrm{~d} t^{2}}=\frac{\mathrm{d} V_{x}}{\mathrm{~d} t}=\left(\frac{S \rho}{2 M}\right)\left(C_{D}\left(v_{x}-V_{x}\right)-\left(C_{L A}\right)\left(v_{y}-V_{y}\right)\right)\left(\left(v_{x}-V_{x}\right)^{2}+\left(v_{y}-V_{y}\right)^{2}\right)^{1 / 2}, \\
& \frac{\mathrm{d}^{2} y}{\mathrm{~d} t^{2}}=\frac{\mathrm{d} V_{y}}{\mathrm{~d} t}=\left(\frac{S \rho}{2 M}\right)\left(C_{D}\left(v_{y}-V_{y}\right)+\left(C_{L A}\right)\left(v_{x}-V_{x}\right)\right)\left(\left(v_{x}-V_{x}\right)^{2}+\left(v_{y}-V_{y}\right)^{2}\right)^{1 / 2}-g, \\
& \frac{\mathrm{d}^{2} \theta}{\mathrm{d} t^{2}}=\frac{\mathrm{d} \omega}{\mathrm{d} t}=\left(\frac{\left(C_{M}+C_{M A}\right) S l \rho}{2 I}\right)\left(\left(v_{x}-V_{x}\right)^{2}+\left(v_{y}-V_{y}\right)^{2}\right) .
\end{aligned}
$$




\subsubsection{Hipótesis}

Se considera como primera hipótesis que los cuerpos poseen baja inercia, por lo que tardan muy poco tiempo en alcanzar la velocidad de autorrotación, de manera tal que si aparece un par instantáneamente $\omega$ toma el valor correspondiente a la autorrotación. Esto hace que la ecuación (38) no sea tenida en cuenta y que al inicio del movimiento $\omega$ alcanza el valor correspondiente al de equilibrio de la velocidad relativa correspondiente.

Por otro lado, se considera que sólo hay viento en sentido horizontal $\left(v_{x}\right)$, por lo que $v_{y}=0$, que todos los cuerpos poseen el mismo peso (como parámetro de comparación), y la misma área promedio.

Los parámetros de entrada para el cálculo de las trayectorias se presentan en la tabla 5.2.

Tabla 5.2. Parámetros de entrada

\begin{tabular}{|c|c|}
\hline Parámetros & Valor \\
\hline Área de referencia & $0,007 \mathrm{~m}^{2}$ \\
\hline Densidad del aire & $1,22 \mathrm{~kg} / \mathrm{m}^{3}$ \\
\hline Velocidad horizontal del viento & $10 \mathrm{~m} / \mathrm{s}$ \\
\hline Velocidad vertical del viento & $0 \mathrm{~m} / \mathrm{s}$ \\
\hline Masa & $0,05 \mathrm{~kg}$ \\
\hline Aceleración de la gravedad & $9,81 \mathrm{~m} / \mathrm{s}^{2}$ \\
\hline
\end{tabular}

Los coeficientes de sustentación $C_{L A}$ y de resistencia $C_{D}$ varían en función de la relación de velocidades $u / v$ según la curva característica de cada cuerpo presentada en el apartado 3.2. Para poder resolver las ecuaciones (36) y (37) se procedió a realizar un ajuste de orden 2 en las curvas características con el fin de conocer la función que determine la mencionada variación,

$$
\begin{aligned}
& C_{L_{A}}=A\left(\frac{u}{v}\right)^{2}+B\left(\frac{u}{v}\right)+C, \\
& C_{D}=D\left(\frac{u}{v}\right)^{2}+E\left(\frac{u}{v}\right)+F .
\end{aligned}
$$

A partir de la publicación [18] y la tabla 5.1 se conoce la variación de $u$ en función de $v$ para los cuerpos en autorrotación, y se reemplazan en las ecuaciones (39) y (40). 


\subsubsection{Cálculo de trayectorias}

Con las consideraciones mencionadas en el apartado anterior y utilizando Software Matlab y su plataforma Simulink se procedió a resolver las ecuaciones de movimiento. En la figura 5.2. se presentan los resultados de las trayectorias de los cuerpos antes mencionados para una distancia horizontal de $1 \mathrm{~m}$.

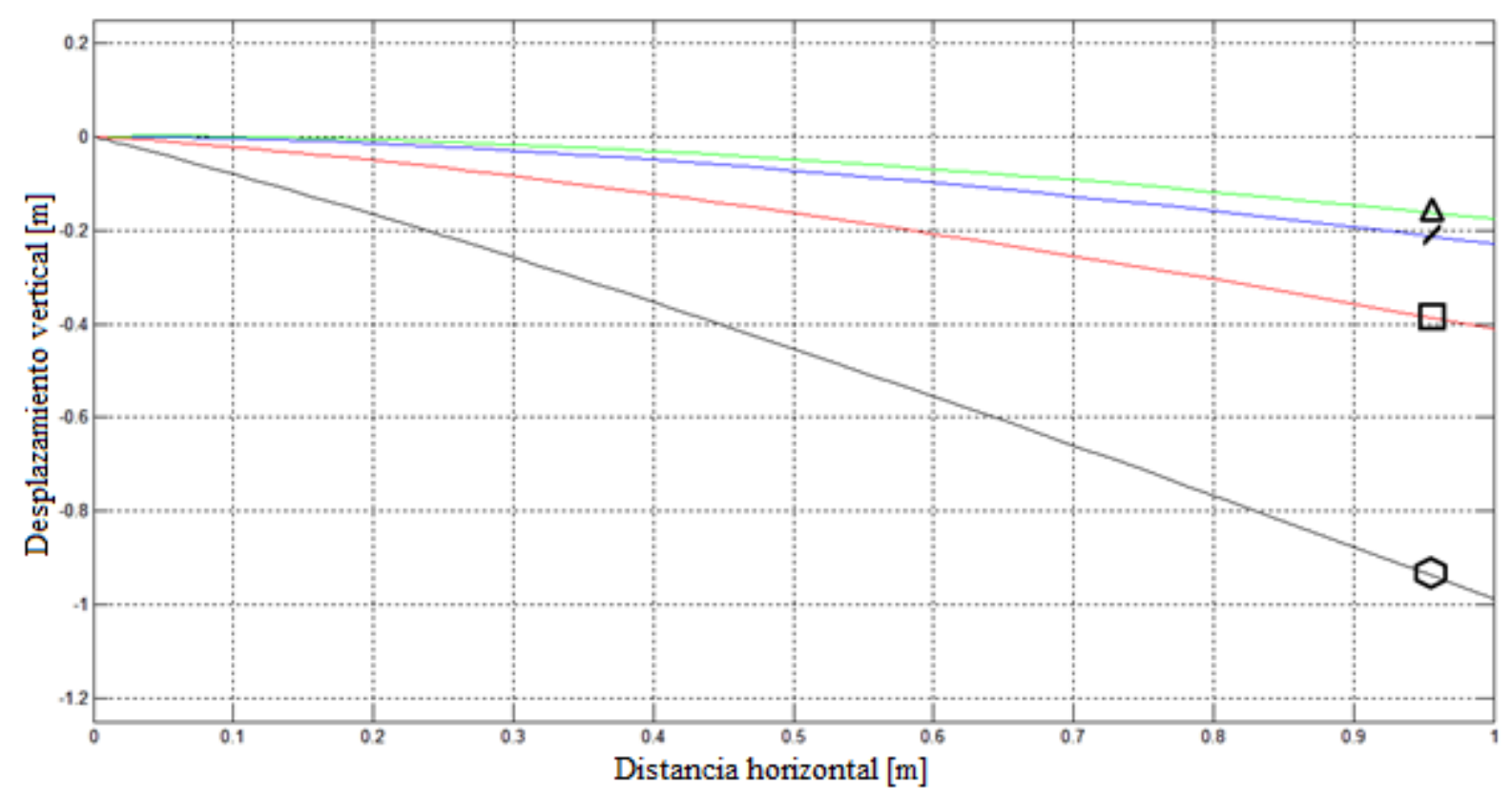

Figura 5.2 Trayectoria de los cuerpos (T, PPF, CD y H) sometidos a una corriente incidente y en autorrotación.

\subsection{CONCLUSIONES}

Como primera conclusión se puede mencionar que la suma de coeficientes de sustentación $\left(C_{L}+C_{L A}\right)$ planteada por Baker [50] no es tenida en cuenta ya que se utilizan cuerpos simétricos en sus dos ejes (eje $x$ y eje $y$ ) y por tal el $C_{L}=0$. Sólo aparece $C_{L A}$ producto de la rotación del cuerpo.

Se puede observar que el triángulo, que es el cuerpo que tiene una relación $u / v$ de autorrotación más alta, es el que menos desciende en la trayectoria. Sin embargo, se observa que por más que el cuadrado posea un valor mayor en la relación $u / v$ de autorrotación que la 
placa plana, este desciende más, ya que la variación de $C_{L}$ en función de $u / v$ es más grande que en el caso de la placa plana.

Así mismo, se observa que el hexágono es el cuerpo que más desciende, ya que es el que menos sustentación genera por la autorrotación.

Este modelo permite estudiar la trayectoria del resto de los cuerpos (T, CD y H) que poseen autorrotación y que no han sido estudiados.

Así mismo, sería de gran utilidad como trabajo futuro, realizar ensayos de autorrotación con los modelos ensayados para verificar las relaciones $u / v$ y poder medir el par generado con el fin de obtener el coeficiente de momentos $C_{M}$. 


\section{CAPÍTULO 6}

\section{CONCLUSIONES}

\subsection{INTRODUCCIÓN}

En el presente capítulo se presenta un resumen de las conclusiones recogidas en cada capítulo componente de la presente tesis, su interrelación, sus aportes novedosos y las futuras líneas de investigación vinculadas a la temática abordada.

Si bien existe mucha información relacionada al efecto Magnus, no se encuentran estudios específicos que brinden la información que presenta esta Tesis en relación a los cuerpos estudiados, los resultados obtenidos en las mediciones de fuerzas en los rangos de velocidades ensayados, las visualizaciones de flujo, el desarrollo teórico y el modelo de estudio de trayectorias.

\subsection{ESTUDIOS EXPERIMENTALES}

La presente Tesis se centra en estudios experimentales, debido a que el estudio analítico del flujo turbulento en el entorno de cuerpos sometidos a un giro y a una corriente es complejo. Por tal se realizaron ensayos en el túnel de viento de circulación cerrada de la Facultad de Ingeniería de la UNLP, lo que permitió determinar la necesidad de construcción y puesta a punto de un túnel de viento más pequeño, que permita ensayar modelos de menor escala donde los problemas de desbalanceo sean más factibles de corregir. Luego de la mencionada puesta a punto y ensayos de prueba se procedió a los ensayos experimentales que son base de la presente Tesis.

Se desarrollaron dos tipos de ensayos, uno correspondiente a mediciones de fuerzas aerodinámicas y otro de visualizaciones de flujo. 
El primero es de gran importancia, ya que aporta datos novedosos a la literatura relacionados al estudio del efecto Magnus en cuerpos cilíndricos no circulares. Gracias a estos ensayos se pueden determinar las fuerzas aerodinámicas (sustentación y resistencia) que se generan producto de la rotación y la corriente de aire, y permiten calcular como varían los coeficientes de sustentación $C_{L}$ y resistencia $C_{D}$ en función de la relación de velocidades $u / v$.

En el caso de los ensayos de visualizaciones de flujo, los mismos permiten conocer los patrones de torbellinos y estela que se generan entorno a los cuerpos. Estos ensayos también presentan un aporte novedoso, ya que muchos de los mismos no se encuentran en la bibliografía y, además, permiten comprender el porqué de las fuerzas que se generan en un cuerpo en rotación.

Gracias a los resultados obtenidos en ambos estudios experimentales se pudo determinar, analizar y comprender el comportamiento aerodinámico de los cuerpos.

\subsection{ANÁLISIS DE RESULTADOS}

El análisis de resultados es de gran importancia en la presente Tesis. Gracias a las fotografías y a los diagramas de flujo realizados, se interpretan los resultados obtenidos en las mediciones de fuerzas de los ensayos experimentales.

Se determina que los cuerpos con menor cantidad de caras poseen mayor sustentación en función del proceso de formación, separación y desprendimiento de los torbellinos generados. Se estudió el comportamiento del flujo en cuerpos con aristas redondeadas y con aristas filosas, que a su vez determina menores valores de $C_{L}$ y mayores de $C_{D}$ en estos últimos.

Los resultados permiten comprender cómo, en función de la posición angular, un cuerpo con aristas en rotación genera diversos torbellinos en las mismas y por tal, en función de la velocidad de giro o de la corriente, como se generan los diversos patrones de desprendimiento y de estela turbulenta. De esta manera, se pueden comprender los resultados obtenidos en los ensayos de medición de fuerzas y el porqué de los diferentes valores según el cuerpo que se estudie. 


\subsection{ESTUDIOS TEÓRICOS}

A partir de definir tiempos de desprendimiento de torbellinos, mediante el uso del número de Strouhal, y tiempos característicos de la variación de la condición de contorno, se ha desarrollado una algoritmo útil para conocer el comportamiento de la sustentación de cuerpos en rotación que posean más de 4 caras. Dicho algoritmo está definido en función de los resultados obtenidos en los ensayos, por lo cual ajusta a los mismos.

Si bien se encuentra acotado a los cuerpos ensayados, el modelo teórico se considera de gran importancia, porque es novedoso, ya que no se encuentra en la bibliografía algún ajuste matemático para cuerpos que posean caras. Por otro lado, no se ha desarrollado el modelo teórico para cuerpos con menos de cuatro caras, ya que el comportamiento fluidodinámico y la generación de torbellinos asociada es compleja y no permite ajustar los criterios utilizados para los otros cuerpos.

\subsection{TRAYECTORIAS}

Este estudio permite analizar, a partir de las características aerodinámicas determinadas en los ensayos, las trayectorias de vuelo de los cuerpos con autorrotación.

La importancia de este capítulo radica en que el modelo desarrollado, a partir de utilizar las ecuaciones de Baker [50] y el planteo de hipótesis iniciales, brinda la posibilidad de ser utilizado para el resto de los cuerpos que poseen autorrotación, que no han sido estudiados en la bibliografía. Si bien para la presente Tesis no se realizaron ensayos de autorrotación, utilizar los resultados de otros autores es válido y sirvió para desarrollar el mencionado modelo.

\subsection{TRABAJOS FUTUROS}

Como futuras líneas de investigación relacionadas directamente con la presente Tesis se pueden mencionar:

Estudio experimental sobre otros cuerpos: $\mathrm{Si}$ bien en el estudio de los cuerpos se contemplaron muchas configuraciones respecto a cantidad de caras, rugosidad y aristas filosas 
o redondeadas, aún quedan cuerpos que no han sido estudiados y que pueden generar un aporte significativo a la temática. Por ejemplo, estos pueden ser un cuerpo de sección pentagonal (siguiendo con la línea de estudio de cuerpos geométricos) o bien cuerpos no cilíndricos de diferentes secciones como ser cuerpos cónicos o piramidales, cuerpos con protuberancias, aletas asimétricas, entre otros. En estos últimos deberá prestarse atención a las posibles desalineaciones y desbalanceos que puedan alterar la lectura de la medición de las fuerzas.

Estudio experimental con otros parámetros de ensayo: Sería de gran importancia realizar ensayos a mayores relaciones de $u / v$ para ampliar el rango del estudio del comportamiento aerodinámico de los cuerpos.

Estudio en frecuencia de oscilaciones de fuerza de Magnus: En la presente Tesis se realizaron mediciones de la fuerza media de Magnus, sin contemplar las oscilaciones producidas por los desprendimientos que se realizan en la estela. Como futura investigación se puede equipar el túnel con una balanza que mida en frecuencia y de esta manera poder conocer el efecto que se genera por la posición del cuerpo y la ubicación y tamaño del torbellino en relación a la fuerza de Magnus.

Estudio de la estela detrás del cuerpo: En la presente Tesis sólo se estudió un segmento de la estela detrás del cuerpo. Como futura investigación se puede estudiar el comportamiento de la misma en un recorrido más extenso y a su vez realizar un estudio, a partir de fotografías, de los torbellinos determinando posición en los ejes $x$ e $y$, y su desarrollo (tamaño e intensidad) a lo largo del recorrido. Para esto debe modificarse el túnel de viento, el sistema de generación de humo y el sistema de luz estroboscópica en conjunto con la toma de fotografías.

Ampliación del desarrollo matemático: El desarrollo matemático realizado en la presente Tesis se encuentra acotado a cuerpos con más de cuatro caras y utiliza valores propios de los ensayos para determinar coeficientes. Como futura línea de investigación se puede analizar la posibilidad de desarrollar un algoritmo que ajuste a cuerpos con menos caras y estudiar la forma de que el mismo se pueda aplicar para cuerpos con diferentes condiciones geométricas respecto a las ensayadas en la presente Tesis.

Estudio de autorrotación: Si bien el fenómeno de la autorrotación se encuentra muy estudiado, sería muy útil estudiarlo en el caso de los cuerpos ensayados en la presente Tesis. 
De esta manera, determinar el coeficiente de momento $C_{M}$, que es de utilidad para el estudio de las trayectorias en vuelo libre, a partir de la medición de par con una balanza para tal fin.

Estudio mediante la utilización de CFD: El efecto Magnus ha sido estudiado mediante técnicas de CFD en cuerpos cilíndricos o esféricos, pero no se halla en la bibliografía estudios de este tipo en cuerpos con caras o aristas. 


\section{ANEXO 1}

\section{RESULTADOS DE LOS ENSAYOS}

\subsection{INTRODUCCIÓN}

El presente anexo exhibe los resultados de los ensayos experimentales realizados en cada uno de los cuerpos mencionados en el apartado 2.4. A partir de los mismos se centraron los estudios de la presente tesis.

\subsection{RESULTADOS}

A continuación se presentan los resultados de los ensayos para cada modelo, de acuerdo al siguiente formato:

- Tabla con resultados de ensayos de sustentación $L$, resistencia $D$, cálculos de coeficientes $C_{L}, C_{D}$ y relación de velocidades $u / v$.

- Gráfico de sustentación, $L$ vs velocidad de la corriente libre, $v$.

- Gráfico de coeficiente de sustentación, $C_{L}$ vs relación de velocidades, $u / v$.

- Gráfico de resistencia, $D$ vs velocidad de la corriente libre, $v$.

- Gráfico de coeficiente de resistencia, $C_{D}$ vs relación de velocidades, $u / v$.

En los ensayos de resistencia $D$ a velocidad de rotación $\omega=0 \mathrm{rpm}$, los cuerpos fueron colocados en la posición angular que se presenta en la tabla A1.1. 
Tabla A1.1 Posición angular de los cuerpos para los ensayos de resistencia $D$ a $\omega=0 \mathrm{rpm}$.

\begin{tabular}{|c|c|}
\hline \multicolumn{2}{|c|}{ Ensayo de resistencia $\boldsymbol{D}$ para $\boldsymbol{\omega}=\mathbf{0} \mathbf{~ r p m}$} \\
\hline Cuerpo & $\begin{array}{c}\text { Posición Angular } \boldsymbol{\theta} \\
{\left[{ }^{\circ}\right]}\end{array}$ \\
\hline CA & 45 \\
\hline CC & 45 \\
\hline O & 0 \\
\hline H & 30 \\
\hline CD & 45 \\
\hline CAR & 45 \\
\hline T & 60 \\
\hline TAR & 60 \\
\hline PPF & 90 \\
\hline PPG & 90 \\
\hline
\end{tabular}

Cabe señalar que los resultados que se presentan a continuación no están corregidos por efectos de bloqueo en el túnel de viento. 


\subsubsection{Circular liso}

Los resultados correspondientes a los ensayos de sustentación y resistencia se presentan en la tabla A1.2.

Tabla A1.2 Resultados correspondiente a los ensayos de sustentación y resistencia.

\begin{tabular}{|c|c|c|c|c|c|c|c|c|}
\hline \multicolumn{9}{|c|}{ Circular liso $(\phi=0,035 \mathrm{~m})$} \\
\hline $\begin{array}{c}\omega \\
{[\mathbf{r p m}]}\end{array}$ & $\begin{array}{c}v \\
{[\mathbf{m} / \mathbf{s}]}\end{array}$ & $\begin{array}{c}L \\
{[\mathbf{N}]}\end{array}$ & $C_{L}$ & $u / v$ & $\begin{array}{c}v \\
{[\mathbf{m} / \mathbf{s}]}\end{array}$ & $\begin{array}{c}D \\
{[\mathrm{~N}]}\end{array}$ & $C_{D}$ & $u / v$ \\
\hline \multirow[t]{4}{*}{0} & - & - & - & - & 5,20 & 0,09 & 0,83 & 0,00 \\
\hline & - & - & - & - & 7,72 & 0,20 & 0,86 & 0,00 \\
\hline & - & - & - & - & 10,16 & 0,35 & 0,86 & 0,00 \\
\hline & - & - & - & - & 11,28 & 0,42 & 0,85 & 0,00 \\
\hline \multirow[t]{4}{*}{1950} & 5,17 & 0,08 & 0,72 & 0,69 & 5,20 & 0,08 & 0,71 & 0,69 \\
\hline & 7,78 & 0,10 & 0,42 & 0,46 & 7,72 & 0,19 & 0,82 & 0,46 \\
\hline & 10,14 & 0,12 & 0,30 & 0,35 & 10,20 & 0,33 & 0,82 & 0,35 \\
\hline & 11,24 & 0,14 & 0,28 & 0,32 & 11,31 & 0,41 & 0,82 & 0,32 \\
\hline \multirow[t]{4}{*}{2310} & 5,17 & 0,09 & 0,86 & 0,82 & 5,20 & 0,07 & 0,67 & 0,81 \\
\hline & 7,73 & 0,12 & 0,51 & 0,55 & 7,72 & 0,18 & 0,76 & 0,55 \\
\hline & 10,14 & 0,14 & 0,35 & 0,42 & 10,16 & 0,32 & 0,79 & 0,42 \\
\hline & 11,24 & 0,16 & 0,32 & 0,38 & 11,28 & 0,40 & 0,81 & 0,38 \\
\hline \multirow[t]{4}{*}{2520} & 5,17 & 0,10 & 0,95 & 0,89 & 5,20 & 0,07 & 0,69 & 0,89 \\
\hline & 7,75 & 0,13 & 0,55 & 0,60 & 7,77 & 0,18 & 0,75 & 0,59 \\
\hline & 10,14 & 0,16 & 0,38 & 0,46 & 10,16 & 0,32 & 0,80 & 0,45 \\
\hline & 11,24 & 0,17 & 0,34 & 0,41 & 11,28 & 0,40 & 0,81 & 0,41 \\
\hline \multirow[t]{4}{*}{2725} & 5,17 & 0,11 & 1,03 & 0,97 & 5,20 & 0,07 & 0,67 & 0,96 \\
\hline & 7,73 & 0,15 & 0,62 & 0,65 & 7,77 & 0,18 & 0,75 & 0,64 \\
\hline & 10,14 & 0,17 & 0,42 & 0,49 & 10,16 & 0,32 & 0,80 & 0,49 \\
\hline & 11,24 & 0,18 & 0,37 & 0,44 & 11,28 & 0,40 & 0,81 & 0,44 \\
\hline
\end{tabular}




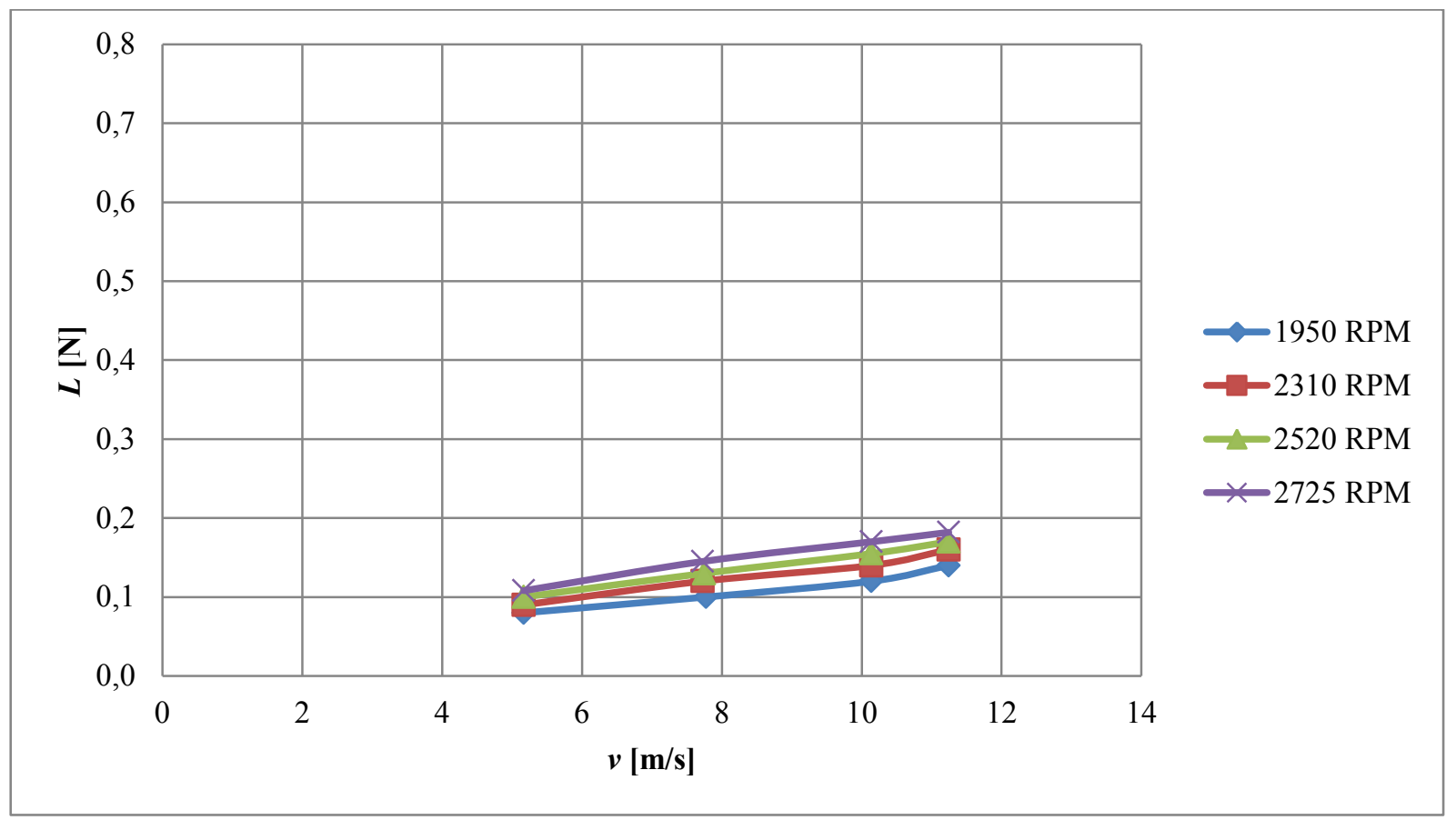

Figura A1.1 Variación de la sustentación $L$ en función de la velocidad de la corriente $v$ para cada velocidad de giro $\omega$.

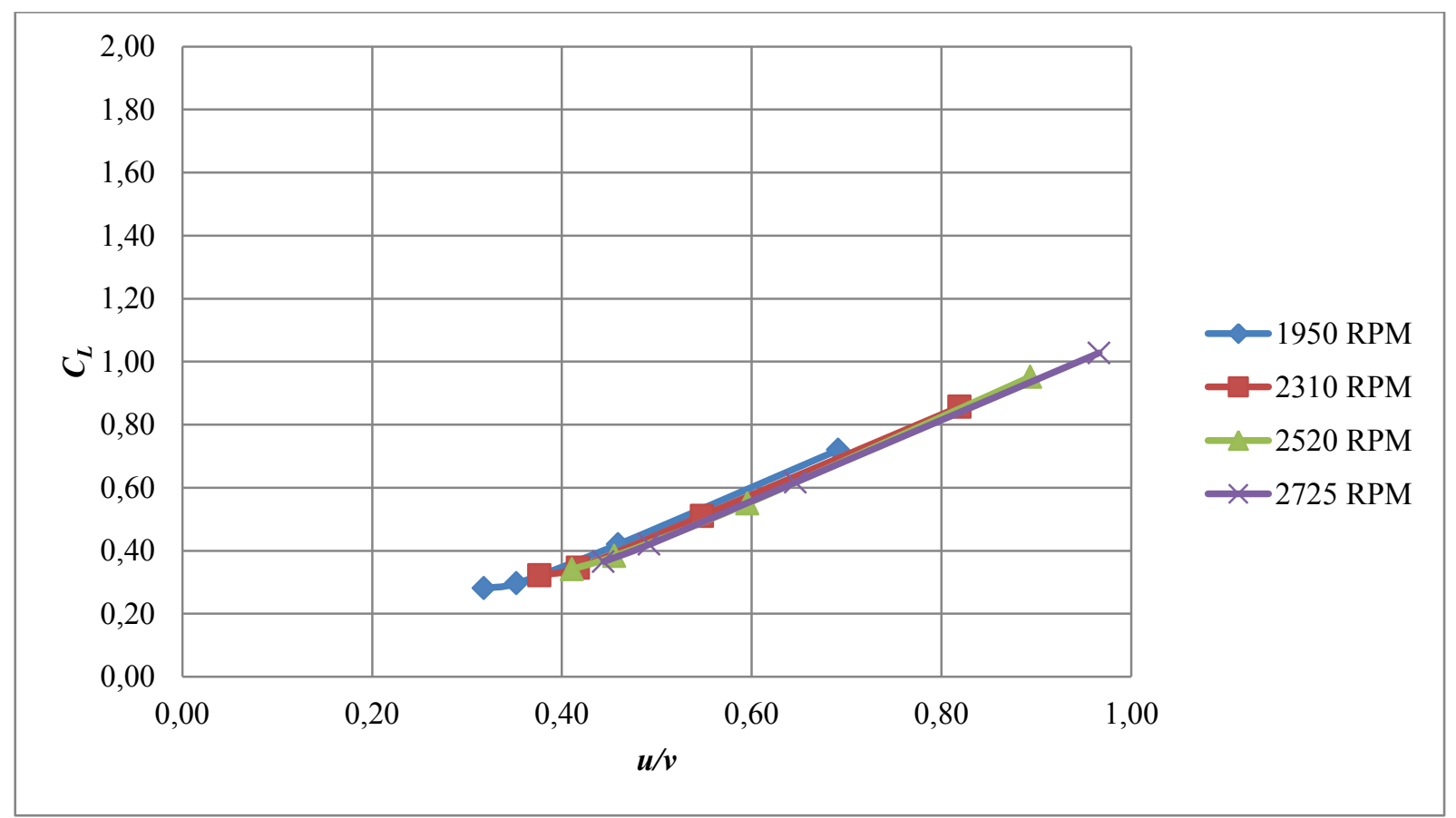

Figura A1.2 Variación del coeficiente de sustentación $C_{L}$ en función de la relación de velocidades $u / v$ para cada velocidad de giro $\omega$. 


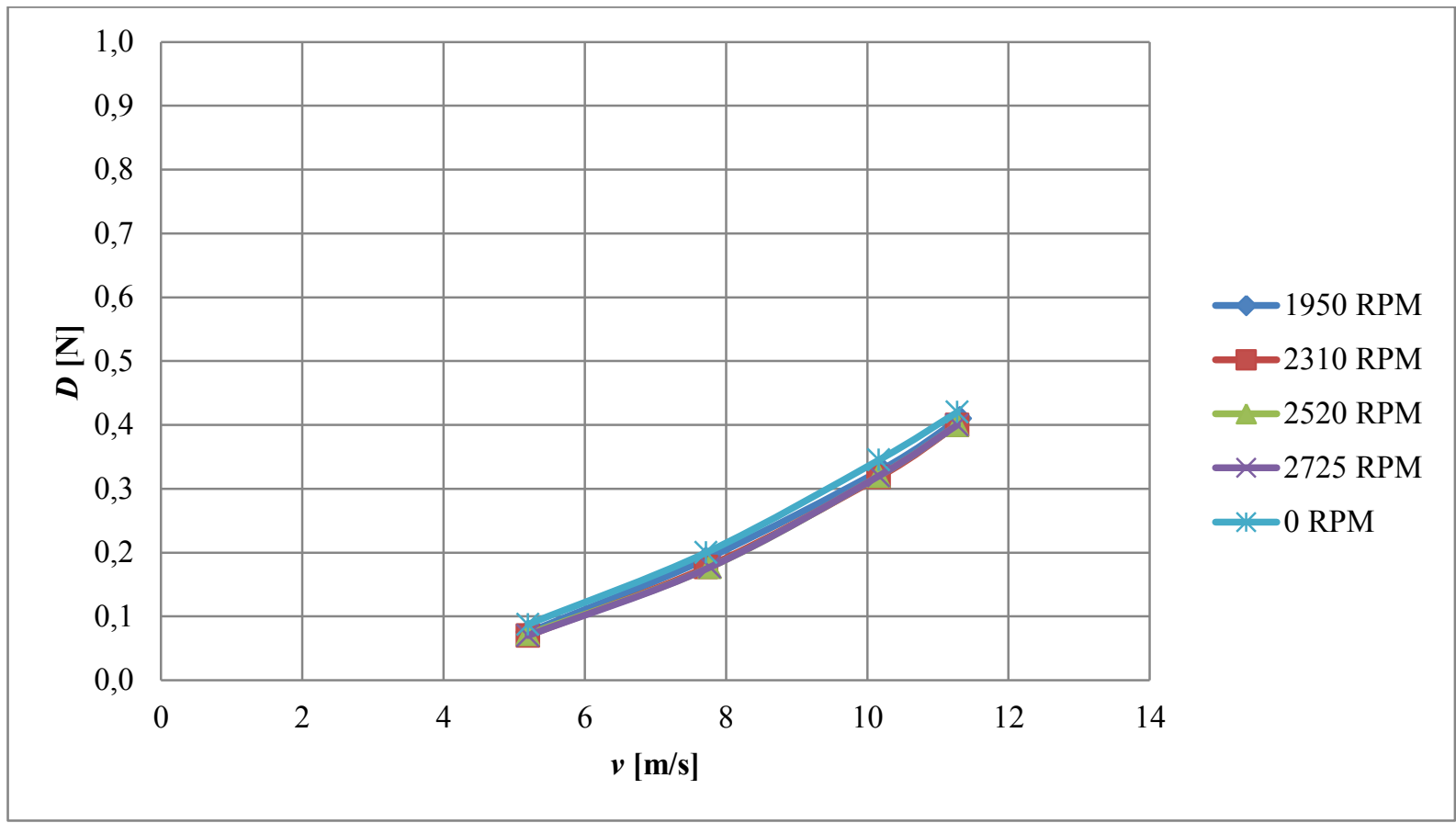

Figura A1.3 Variación de la resistencia $D$ en función de la velocidad de la corriente $v$ para cada velocidad de giro $\omega$.

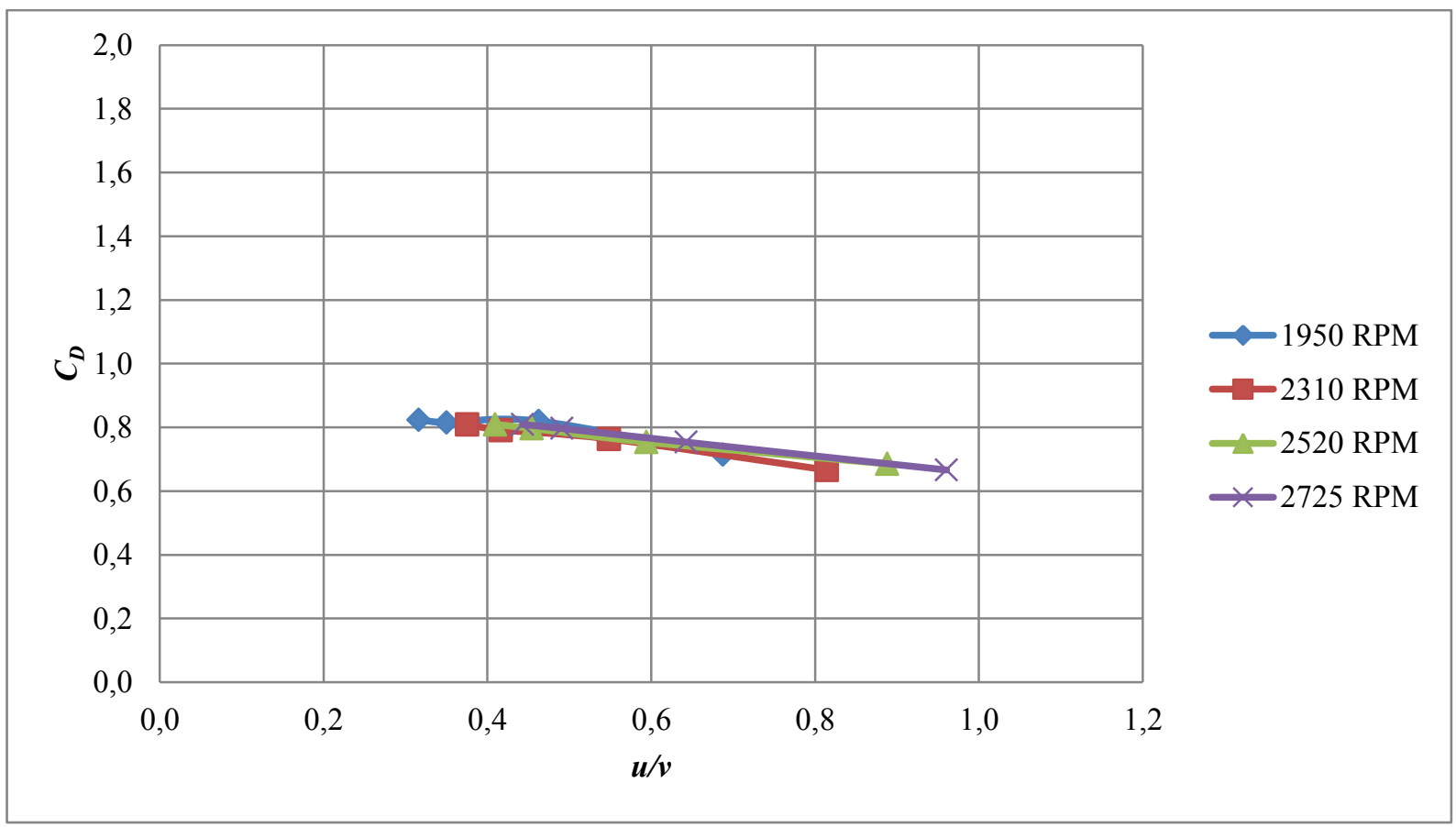

Figura A1.4 Variación del coeficiente de resistencia $C_{D}$ en función de la relación de velocidades $u / v$ para cada velocidad de giro $\omega$. 


\subsubsection{Circular recubierto con lija fina}

Los resultados correspondientes a los ensayos de sustentación y resistencia se presentan en la tabla A1.3.

Tabla A1.3 Resultados correspondiente a los ensayos de sustentación y resistencia.

\begin{tabular}{|c|c|c|c|c|c|c|c|c|}
\hline \multicolumn{9}{|c|}{ Circular recubierto con lija fina $(\phi=0,035 \mathrm{~m})$} \\
\hline $\begin{array}{c}\omega \\
{[\mathbf{r p m}]}\end{array}$ & $\begin{array}{c}v \\
{[\mathbf{m} / \mathbf{s}]}\end{array}$ & $\begin{array}{c}L \\
{[\mathbf{N}]}\end{array}$ & $C_{L}$ & $u / v$ & $\begin{array}{c}v \\
{[\mathbf{m} / \mathbf{s}]}\end{array}$ & $\begin{array}{c}D \\
{[\mathbf{N}]}\end{array}$ & $C_{D}$ & $u / v$ \\
\hline \multirow[t]{4}{*}{0} & - & - & - & - & 5,34 & 0,09 & 0,84 & 0,00 \\
\hline & - & - & - & - & 7,97 & 0,21 & 0,88 & 0,00 \\
\hline & - & - & - & - & 10,56 & 0,37 & 0,87 & 0,00 \\
\hline & - & - & - & - & 11,85 & 0,48 & 0,91 & 0,00 \\
\hline \multirow[t]{4}{*}{1950} & 5,18 & 0,08 & 0,80 & 0,69 & 5,34 & 0,10 & 0,94 & 0,67 \\
\hline & 7,81 & 0,10 & 0,44 & 0,46 & 7,92 & 0,23 & 0,98 & 0,45 \\
\hline & 10,46 & 0,13 & 0,32 & 0,34 & 10,56 & 0,40 & 0,95 & 0,34 \\
\hline & 11,77 & 0,14 & 0,27 & 0,30 & 11,85 & 0,48 & 0,91 & 0,30 \\
\hline \multirow[t]{4}{*}{2310} & & 0,10 & 0,93 & 0,81 & 5,26 & 0,09 & 0,82 & 0,80 \\
\hline & 7,87 & 0,15 & 0,65 & 0,54 & 7,92 & 0,24 & 1,00 & 0,53 \\
\hline & 10,55 & 0,17 & 0,40 & 0,40 & 10,56 & 0,40 & 0,95 & 0,40 \\
\hline & 11,84 & 0,18 & 0,34 & 0,36 & 11,81 & 0,49 & 0,93 & 0,36 \\
\hline \multirow[t]{4}{*}{2520} & 5,22 & 0,11 & 1,03 & 0,88 & 5,30 & 0,09 & 0,81 & 0,87 \\
\hline & 7,92 & 0,17 & 0,72 & 0,58 & 7,92 & 0,23 & 0,98 & 0,58 \\
\hline & 10,55 & 0,19 & 0,44 & 0,44 & 10,60 & 0,40 & 0,95 & 0,44 \\
\hline & 11,81 & 0,20 & 0,38 & 0,39 & 11,85 & 0,51 & 0,96 & 0,39 \\
\hline \multirow[t]{4}{*}{2725} & 5,26 & 0,11 & 1,06 & 0,95 & 5,38 & 0,08 & 0,74 & 0,93 \\
\hline & 7,92 & 0,18 & 0,75 & 0,63 & 7,92 & 0,22 & 0,92 & 0,63 \\
\hline & 10,55 & 0,20 & 0,47 & 0,47 & 10,60 & 0,38 & 0,89 & 0,47 \\
\hline & 11,83 & 0,21 & 0,39 & 0,42 & 11,85 & 0,46 & 0,88 & 0,42 \\
\hline
\end{tabular}




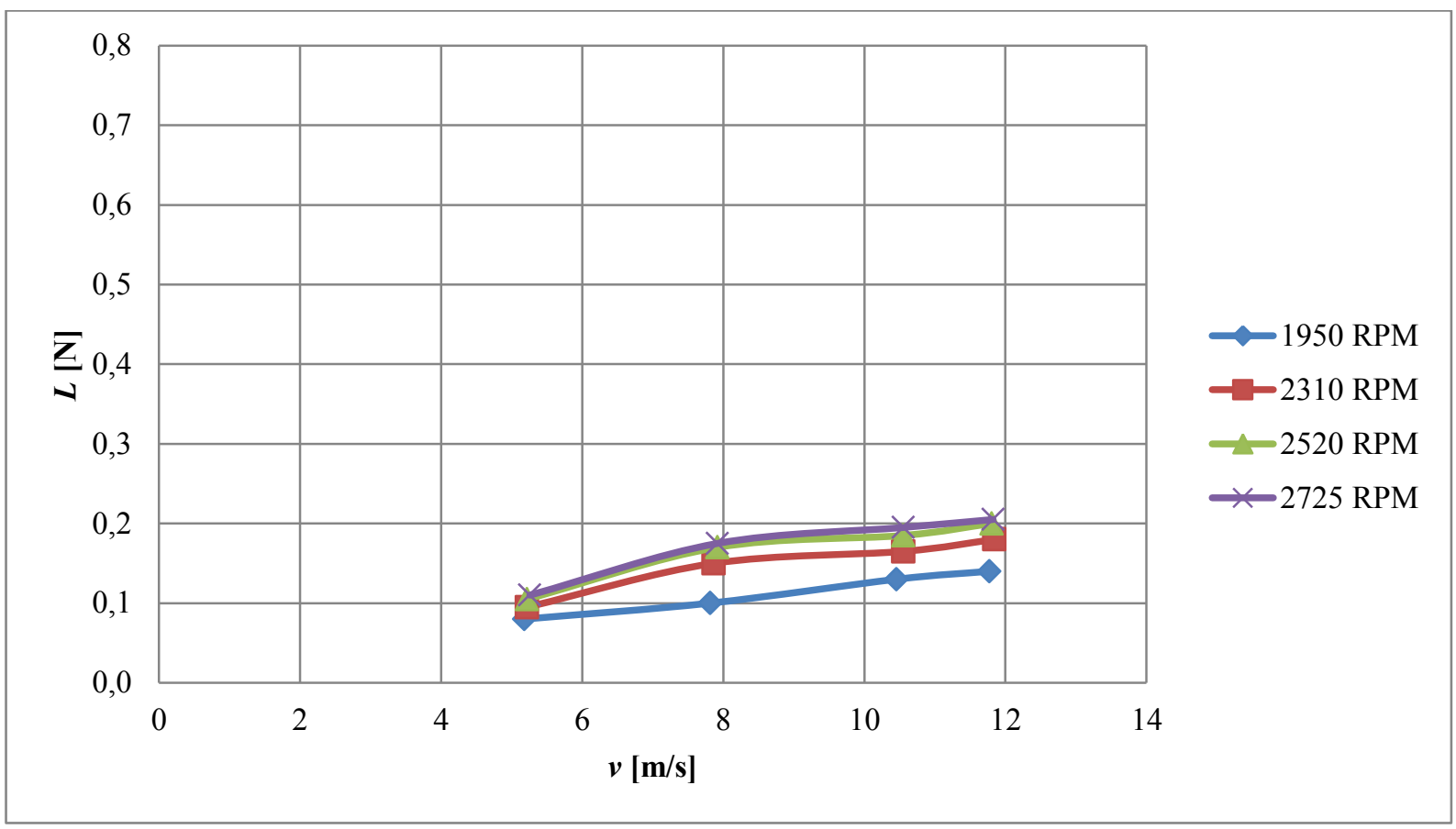

Figura A1.5 Variación de la sustentación $L$ en función de la velocidad de la corriente $v$ para cada velocidad de giro $\omega$.

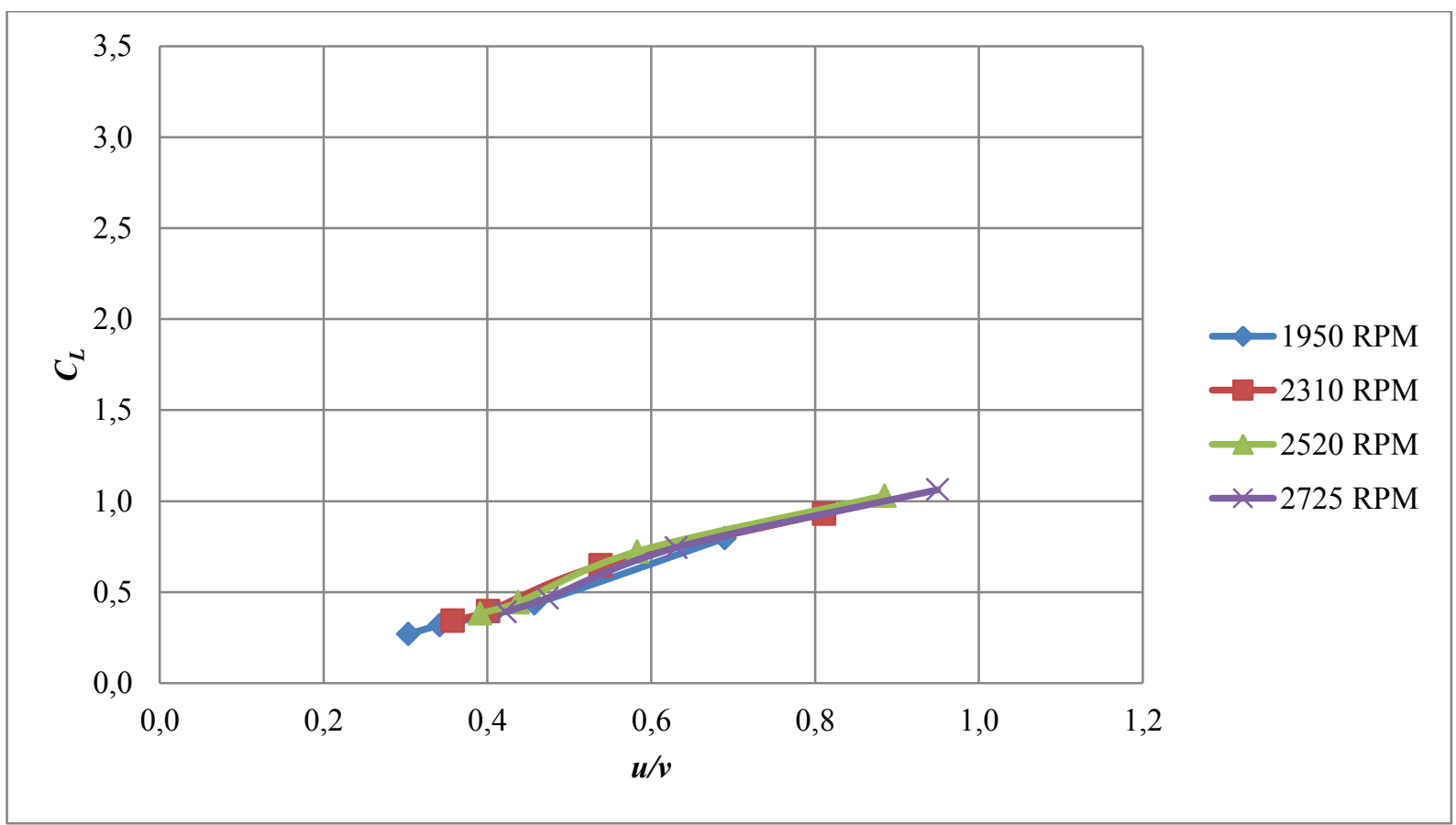

Figura A1.6 Variación del coeficiente de sustentación $C_{L}$ en función de la relación de velocidades $u / v$ para cada velocidad de giro $\omega$. 


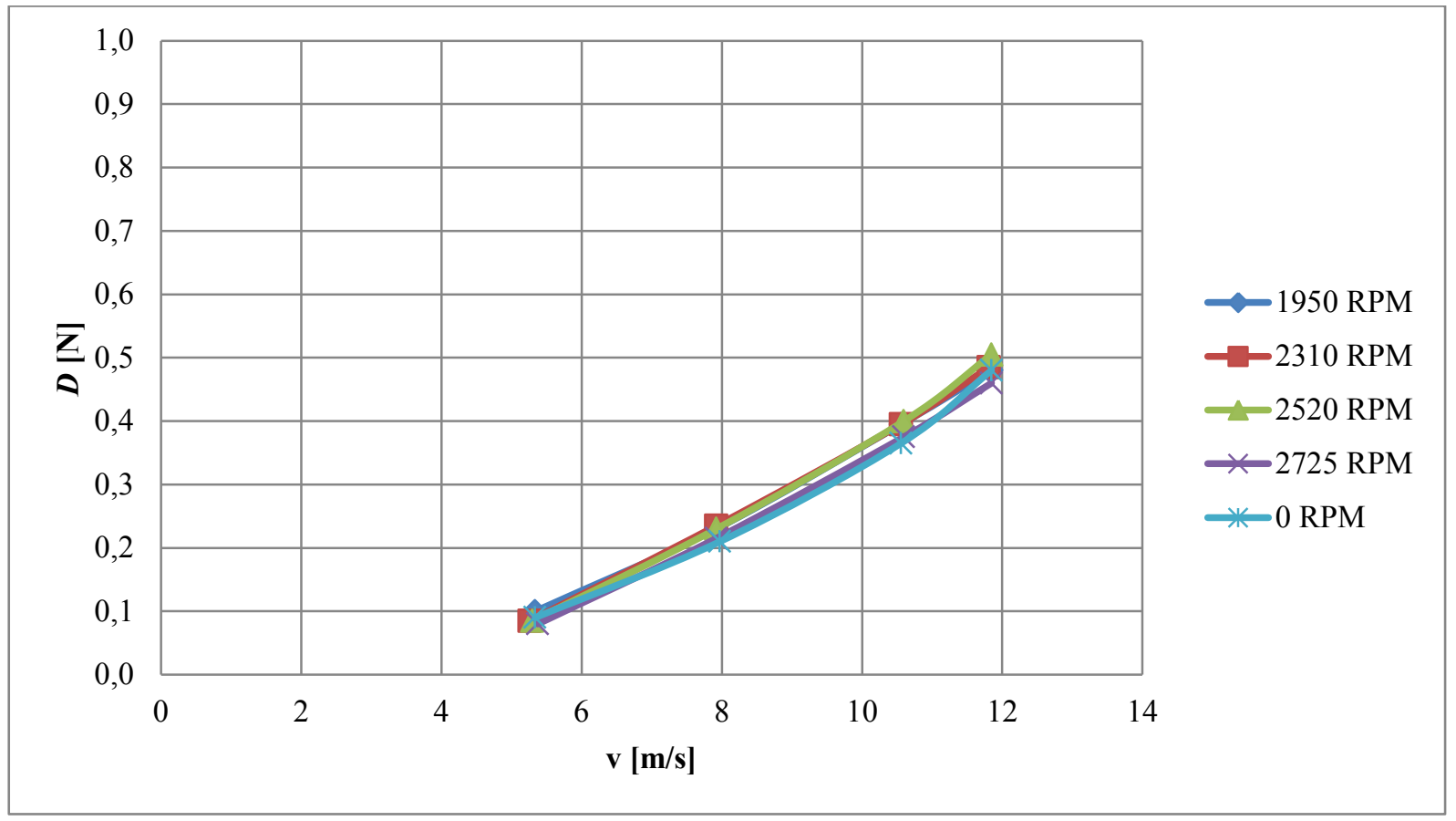

Figura A1.7 Variación de la resistencia $D$ en función de la velocidad de la corriente $v$ para cada velocidad de giro $\omega$.

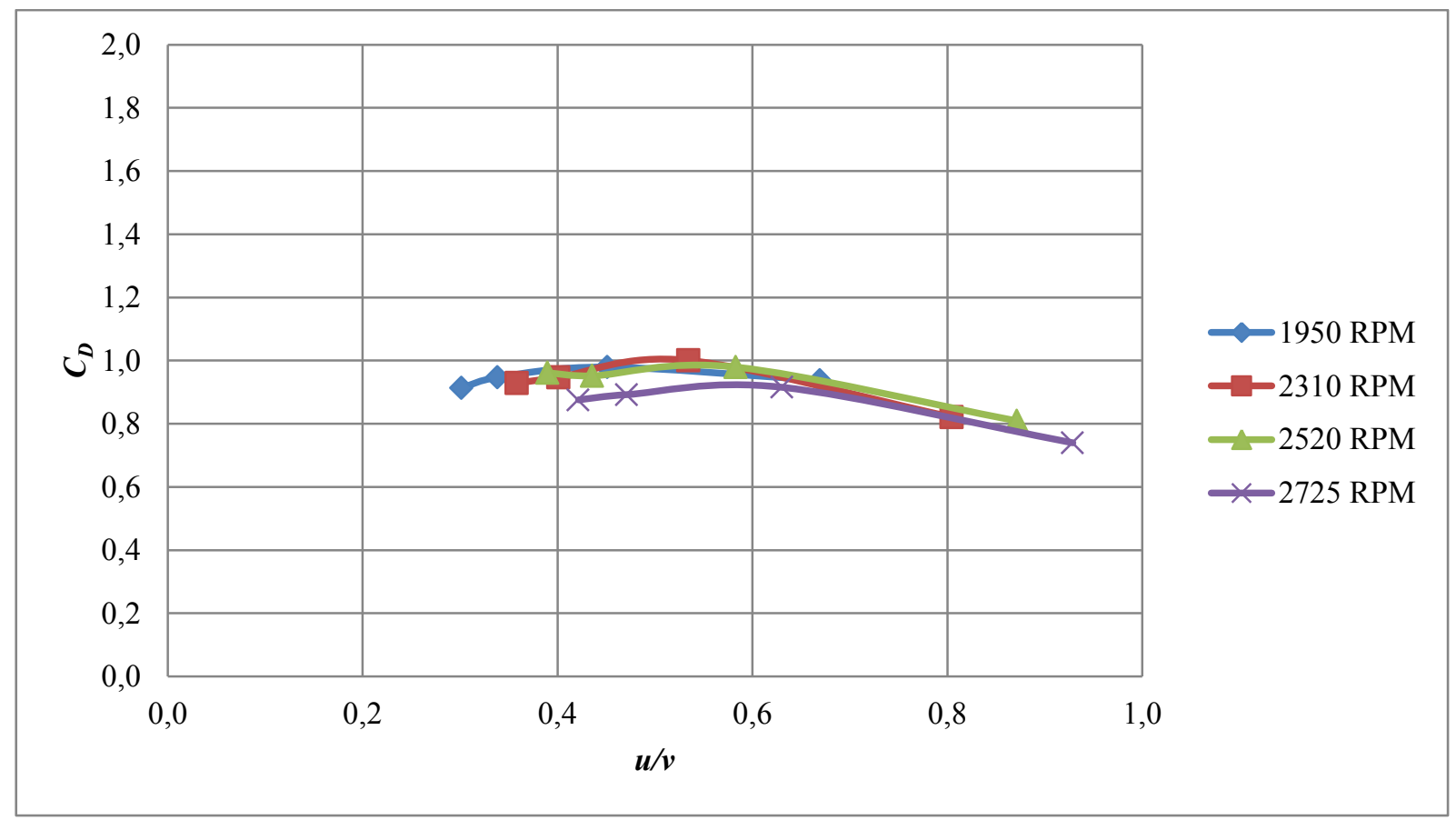

Figura A1.8 Variación del coeficiente de resistencia $C_{\boldsymbol{D}}$ en función de la relación de velocidades $u / v$ para cada velocidad de giro $\omega$. 


\subsubsection{Circular recubierto con lija gruesa}

Los resultados correspondientes a los ensayos de sustentación y resistencia se presentan en la tabla A1.4.

Tabla A1.4 Resultados correspondiente a los ensayos de sustentación y resistencia.

\begin{tabular}{|c|c|c|c|c|c|c|c|c|}
\hline \multicolumn{9}{|c|}{ Circular recubierto con lija gruesa $(\phi=0,035 \mathrm{~m})$} \\
\hline $\begin{array}{c}\omega \\
{[\mathbf{r p m}]}\end{array}$ & $\begin{array}{c}v \\
{[\mathbf{m} / \mathbf{s}]}\end{array}$ & $\begin{array}{c}L \\
{[\mathbf{N}]}\end{array}$ & $C_{L}$ & $u / v$ & $\begin{array}{c}v \\
{[\mathbf{m} / \mathbf{s}]}\end{array}$ & $\begin{array}{c}D \\
{[\mathbf{N}]}\end{array}$ & $C_{D}$ & $u / v$ \\
\hline \multirow[t]{4}{*}{0} & - & - & - & - & 5,13 & 0,09 & 0,88 & 0,00 \\
\hline & - & - & - & - & 7,70 & 0,21 & 0,91 & 0,00 \\
\hline & - & - & - & - & 10,16 & 0,36 & 0,90 & 0,00 \\
\hline & - & - & - & - & 11,27 & 0,43 & 0,88 & 0,00 \\
\hline \multirow[t]{4}{*}{1950} & 5,10 & 0,06 & 0,59 & 0,70 & 5,20 & 0,09 & 0,81 & 0,69 \\
\hline & 7,73 & 0,10 & 0,43 & 0,46 & 7,78 & 0,19 & 0,81 & 0,46 \\
\hline & 10,15 & 0,12 & 0,30 & 0,35 & 10,22 & 0,33 & 0,80 & 0,35 \\
\hline & 11,26 & 0,13 & & & & 0,42 & 0,85 & 0,32 \\
\hline \multirow[t]{4}{*}{2310} & 5,17 & 0,08 & 0,76 & 0,82 & 5,20 & 0,08 & 0,71 & 0,81 \\
\hline & 7,73 & 0,12 & 0,51 & 0,55 & 7,73 & 0,18 & 0,78 & 0,55 \\
\hline & 10,15 & 0,16 & 0,38 & 0,42 & 10,18 & 0,32 & 0,80 & 0,42 \\
\hline & 11,26 & 0,16 & 0,32 & 0,38 & 11,29 & 0,40 & 0,81 & 0,37 \\
\hline \multirow[t]{4}{*}{2520} & 5,17 & 0,10 & 0,95 & 0,89 & 5,20 & 0,08 & 0,71 & 0,89 \\
\hline & 7,73 & 0,15 & 0,62 & 0,60 & 7,76 & 0,19 & 0,79 & 0,60 \\
\hline & 10,15 & 0,18 & 0,43 & 0,45 & 10,18 & 0,32 & 0,80 & 0,45 \\
\hline & 11,26 & 0,20 & 0,39 & 0,41 & 11,29 & 0,41 & 0,82 & 0,41 \\
\hline \multirow[t]{4}{*}{2725} & 0 & 0,00 & 0,00 & 0,00 & 5,20 & 0,08 & 0,71 & 0,96 \\
\hline & 5,17 & 0,12 & 1,09 & 0,97 & 7,73 & 0,19 & 0,82 & 0,65 \\
\hline & 7,73 & 0,18 & 0,75 & 0,65 & 10,16 & 0,32 & 0,79 & 0,49 \\
\hline & 10,15 & 0,20 & 0,49 & 0,49 & 11,31 & 0,39 & 0,79 & 0,44 \\
\hline
\end{tabular}




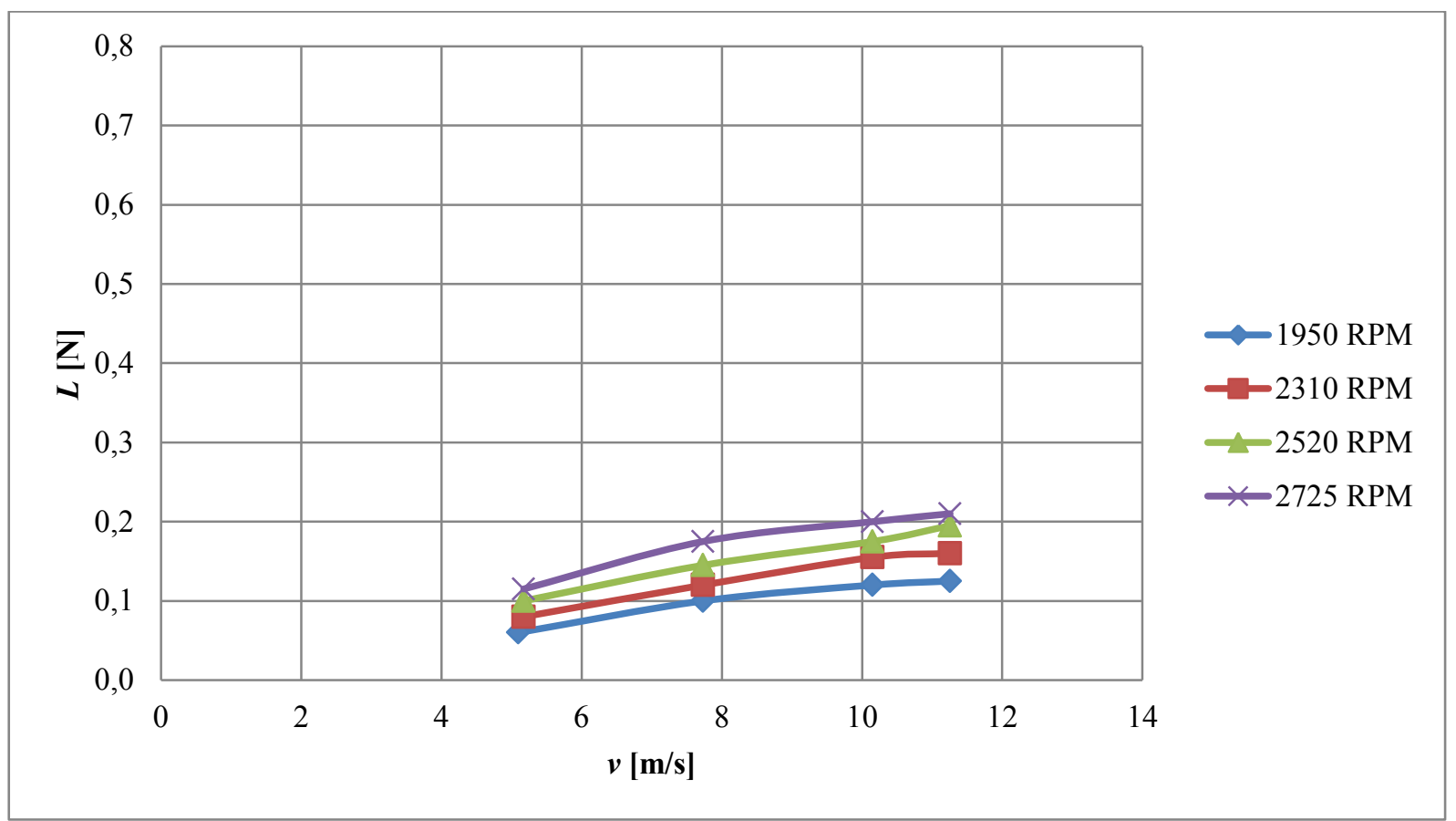

Figura A1.9 Variación de la sustentación $L$ en función de la velocidad de la corriente $v$ para cada velocidad de giro $\omega$.

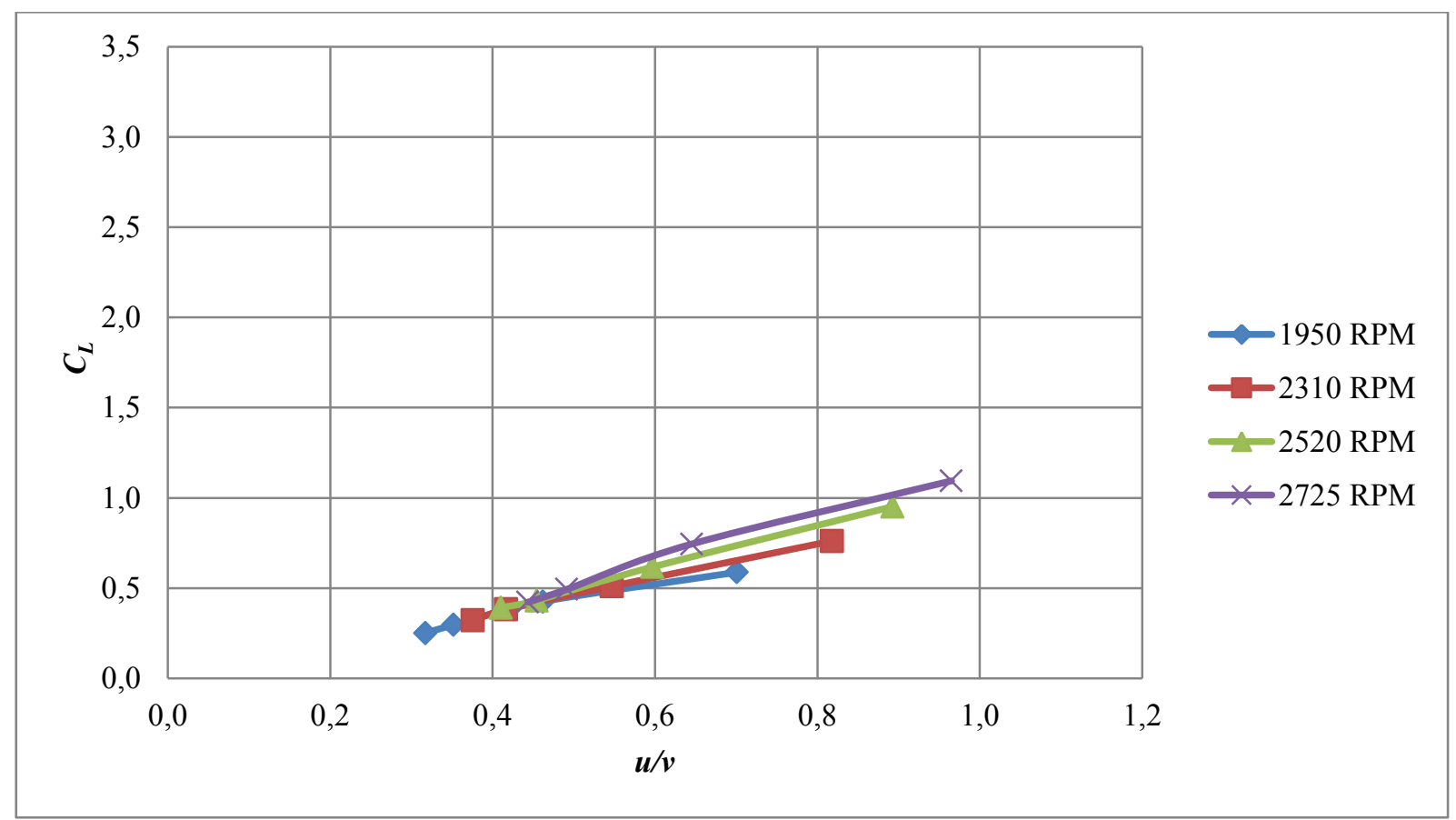

Figura A1.10 Variación del coeficiente de sustentación $C_{L}$ en función de la relación de velocidades $u / v$ para cada velocidad de giro $\omega$. 


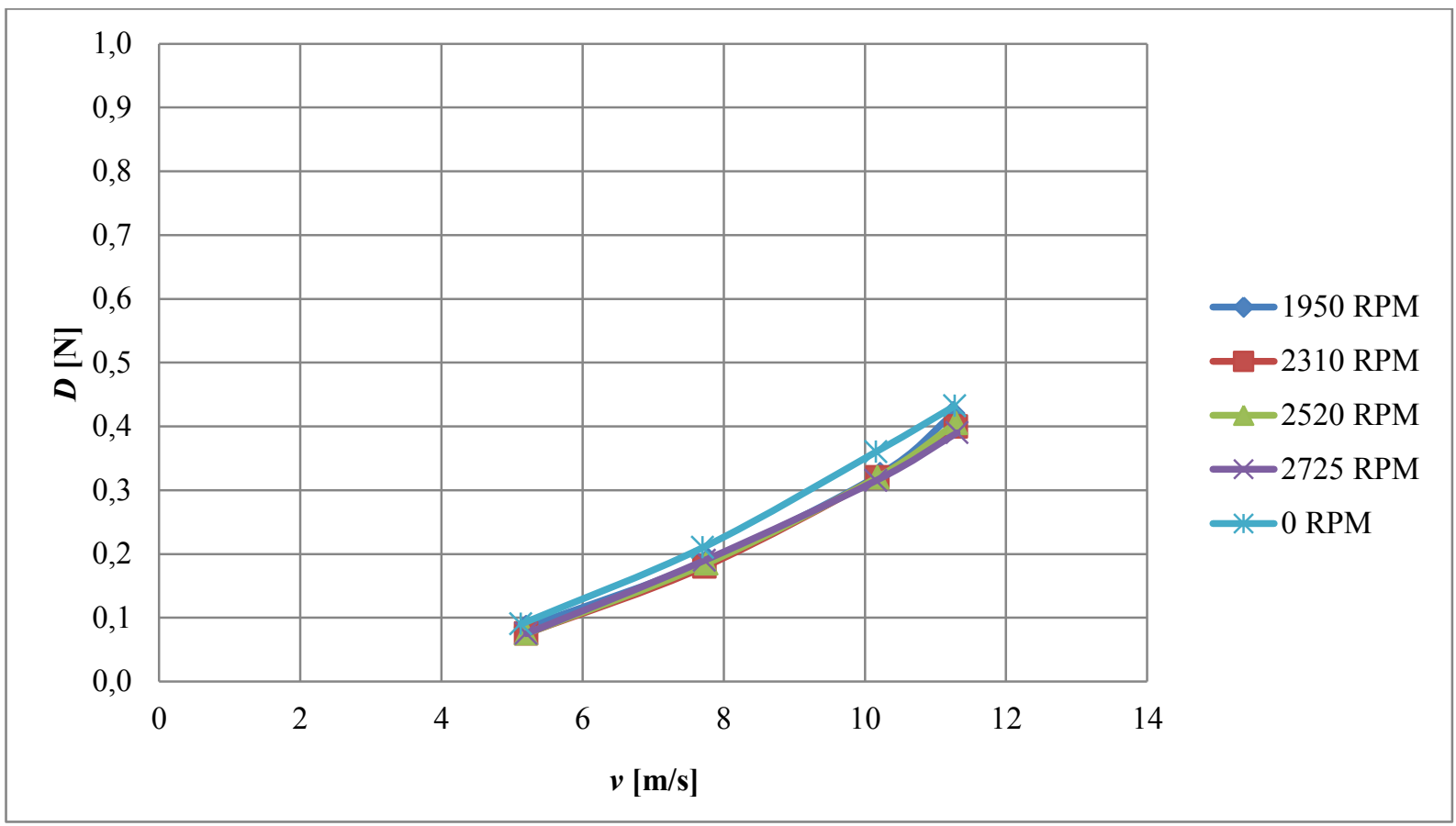

Figura A1.11 Variación de la resistencia $D$ en función de la velocidad de la corriente $v$ para cada velocidad de giro $\omega$.

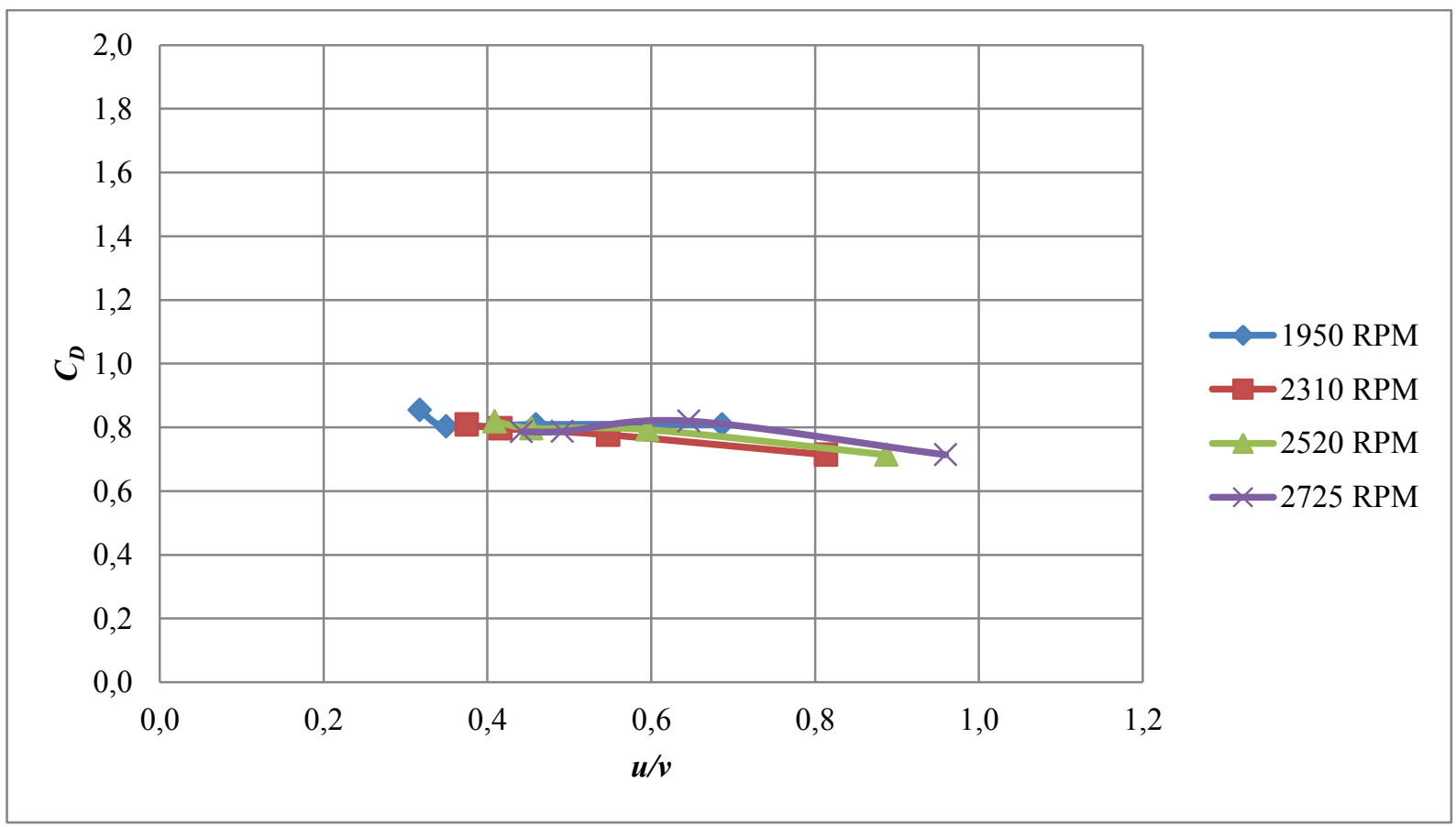

Figura A1.12 Variación del coeficiente de resistencia $C_{\boldsymbol{D}}$ en función de la relación de velocidades $u / v$ para cada velocidad de giro $\omega$. 


\subsubsection{Circular calado}

Los resultados correspondientes a los ensayos de sustentación y resistencia se presentan en la tabla A1.5.

Tabla A1.5 Resultados correspondiente a los ensayos de sustentación y resistencia.

\begin{tabular}{|c|c|c|c|c|c|c|c|c|}
\hline \multicolumn{9}{|c|}{ Circular calado $(\phi=0,035 \mathrm{~m})$} \\
\hline $\begin{array}{c}\omega \\
{[\mathbf{r p m}]}\end{array}$ & $\begin{array}{c}v \\
{[\mathbf{m} / \mathbf{s}]}\end{array}$ & $\begin{array}{c}L \\
{[\mathbf{N}]}\end{array}$ & $C_{L}$ & $u / v$ & $\begin{array}{c}v \\
{[\mathbf{m} / \mathbf{s}]}\end{array}$ & $\begin{array}{c}D \\
{[\mathbf{N}]}\end{array}$ & $C_{D}$ & $u / v$ \\
\hline \multirow[t]{4}{*}{0} & - & - & - & - & 5,29 & 0,09 & 0,81 & 0,00 \\
\hline & - & - & - & - & 7,96 & 0,21 & 0,86 & 0,00 \\
\hline & - & - & - & - & 10,55 & 0,37 & 0,87 & 0,00 \\
\hline & - & - & - & - & 11,82 & 0,46 & 0,88 & 0,00 \\
\hline \multirow[t]{4}{*}{1950} & 5,16 & 0,10 & 0,96 & 0,69 & 5,29 & 0,08 & 0,76 & 0,68 \\
\hline & 7,91 & 0,21 & 0,88 & 0,45 & 7,96 & 0,21 & 0,86 & 0,45 \\
\hline & 10,53 & 0,35 & 0,85 & 0,34 & 10,57 & 0,37 & 0,87 & 0,34 \\
\hline & 11,87 & 0,40 & 0,77 & 0,30 & 11,86 & 0,47 & 0,89 & 0,30 \\
\hline \multirow[t]{4}{*}{2310} & 5,20 & 0,10 & 1,00 & 0,81 & 5,29 & 0,08 & 0,71 & 0,80 \\
\hline & 7,91 & 0,22 & 0,93 & 0,54 & 7,96 & 0,20 & 0,82 & 0,53 \\
\hline & 10,51 & 0,37 & 0,89 & 0,40 & 10,53 & 0,36 & 0,86 & 0,40 \\
\hline & 11,78 & 0,42 & 0,81 & 0,36 & 11,79 & 0,45 & 0,86 & 0,36 \\
\hline \multirow[t]{4}{*}{2520} & 5,16 & 0,10 & 1,01 & 0,89 & 5,29 & 0,07 & 0,67 & 0,87 \\
\hline & 7,91 & 0,21 & 0,88 & 0,58 & 7,96 & 0,19 & 0,80 & 0,58 \\
\hline & 10,51 & 0,36 & 0,87 & 0,44 & 10,53 & 0,35 & 0,84 & 0,44 \\
\hline & 11,87 & 0,42 & 0,79 & 0,39 & 11,82 & 0,44 & 0,84 & 0,39 \\
\hline \multirow[t]{4}{*}{2725} & 5,20 & 0,11 & 1,10 & 0,96 & 5,29 & 0,09 & 0,86 & 0,94 \\
\hline & 7,91 & 0,22 & 0,93 & 0,63 & 7,96 & 0,22 & 0,90 & 0,63 \\
\hline & 10,51 & 0,36 & 0,87 & 0,48 & 10,65 & 0,39 & 0,91 & 0,47 \\
\hline & 11,76 & 0,41 & 0,80 & 0,42 & 11,82 & 0,48 & 0,91 & 0,42 \\
\hline
\end{tabular}




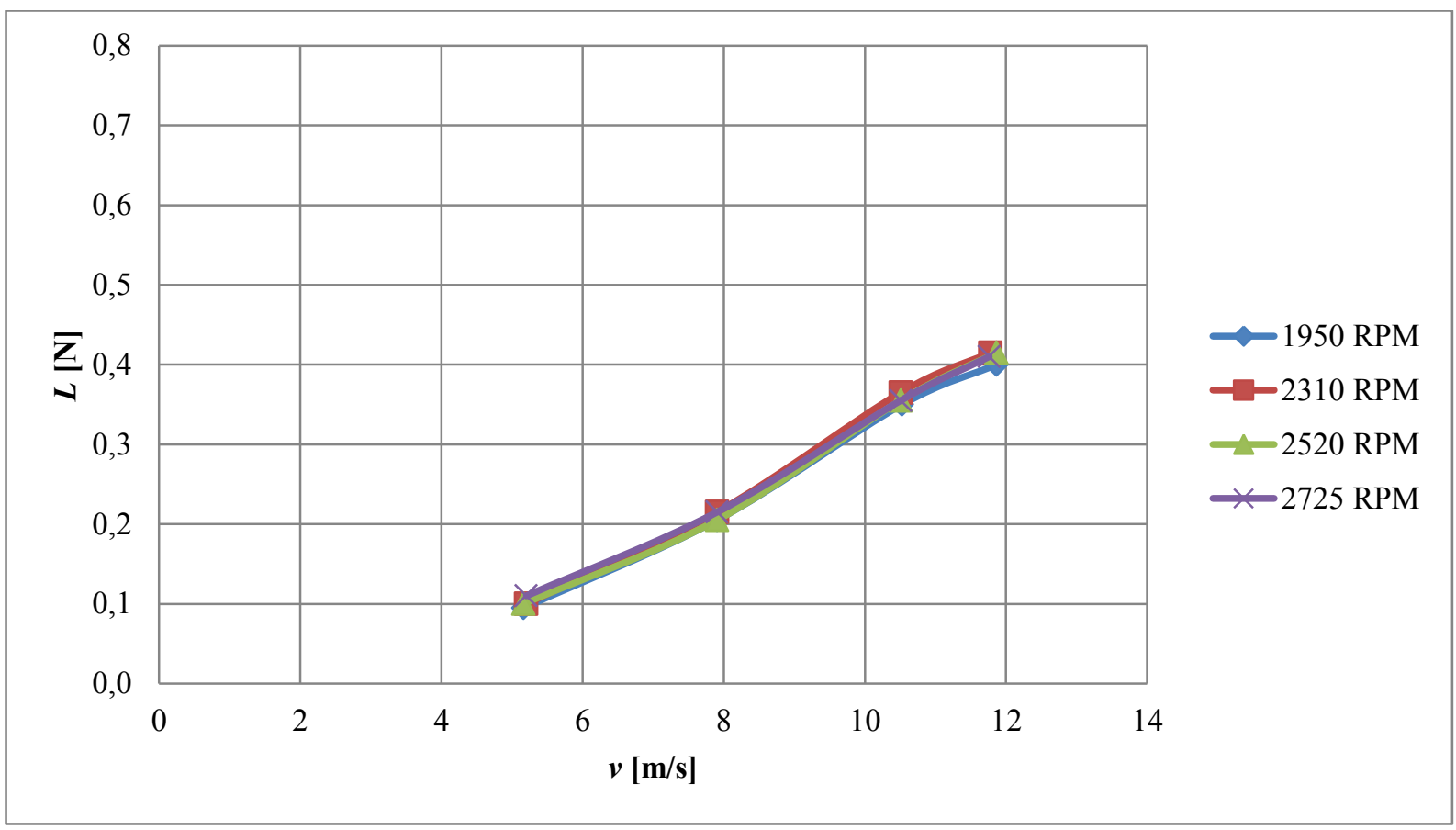

Figura A1.13 Variación de la sustentación $L$ en función de la velocidad de la corriente $v$ para cada velocidad de giro $\omega$.

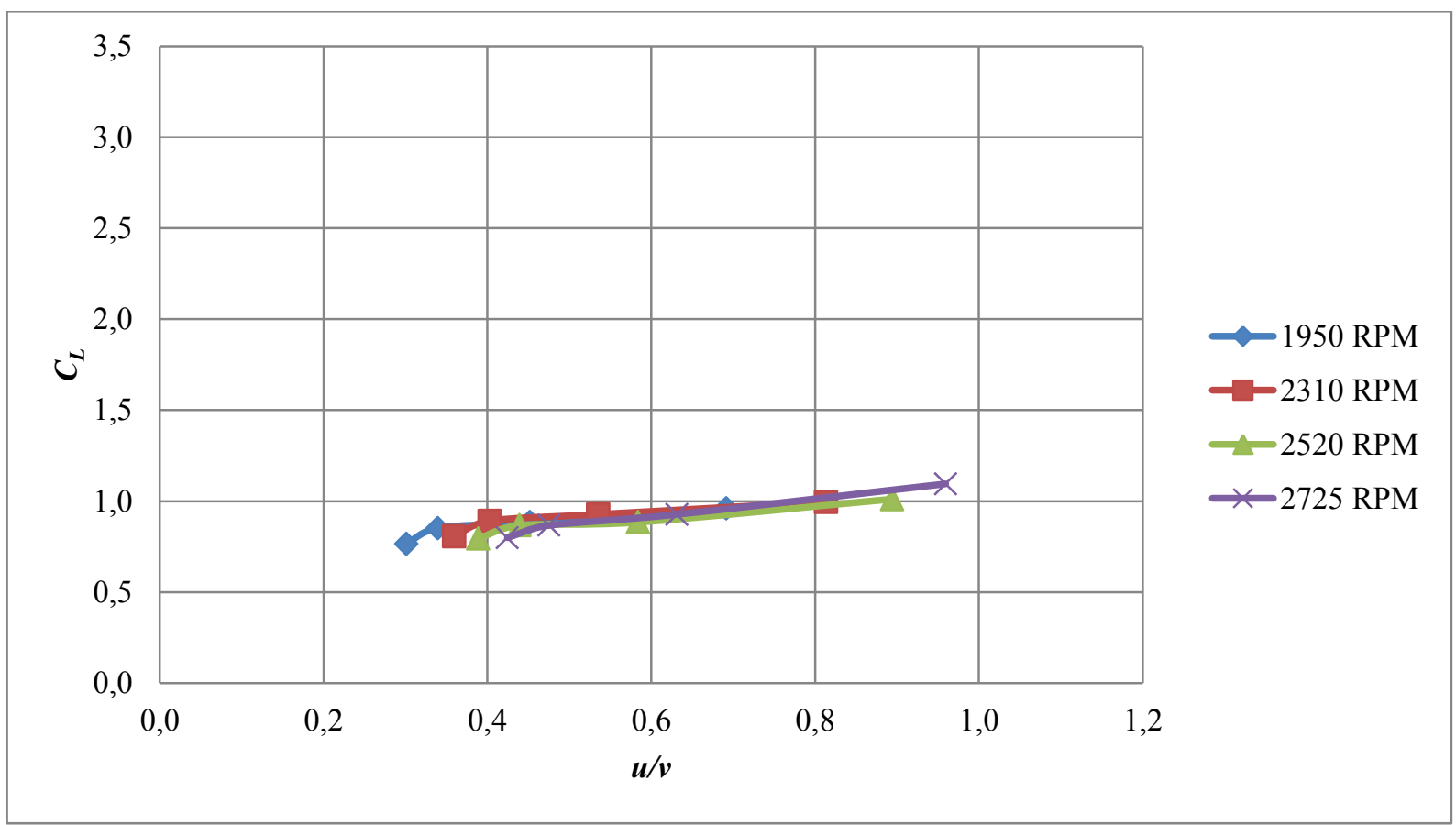

Figura A1.14 Variación del coeficiente de sustentación $C_{L}$ en función de la relación de velocidades $u / v$ para cada velocidad de giro $\omega$. 


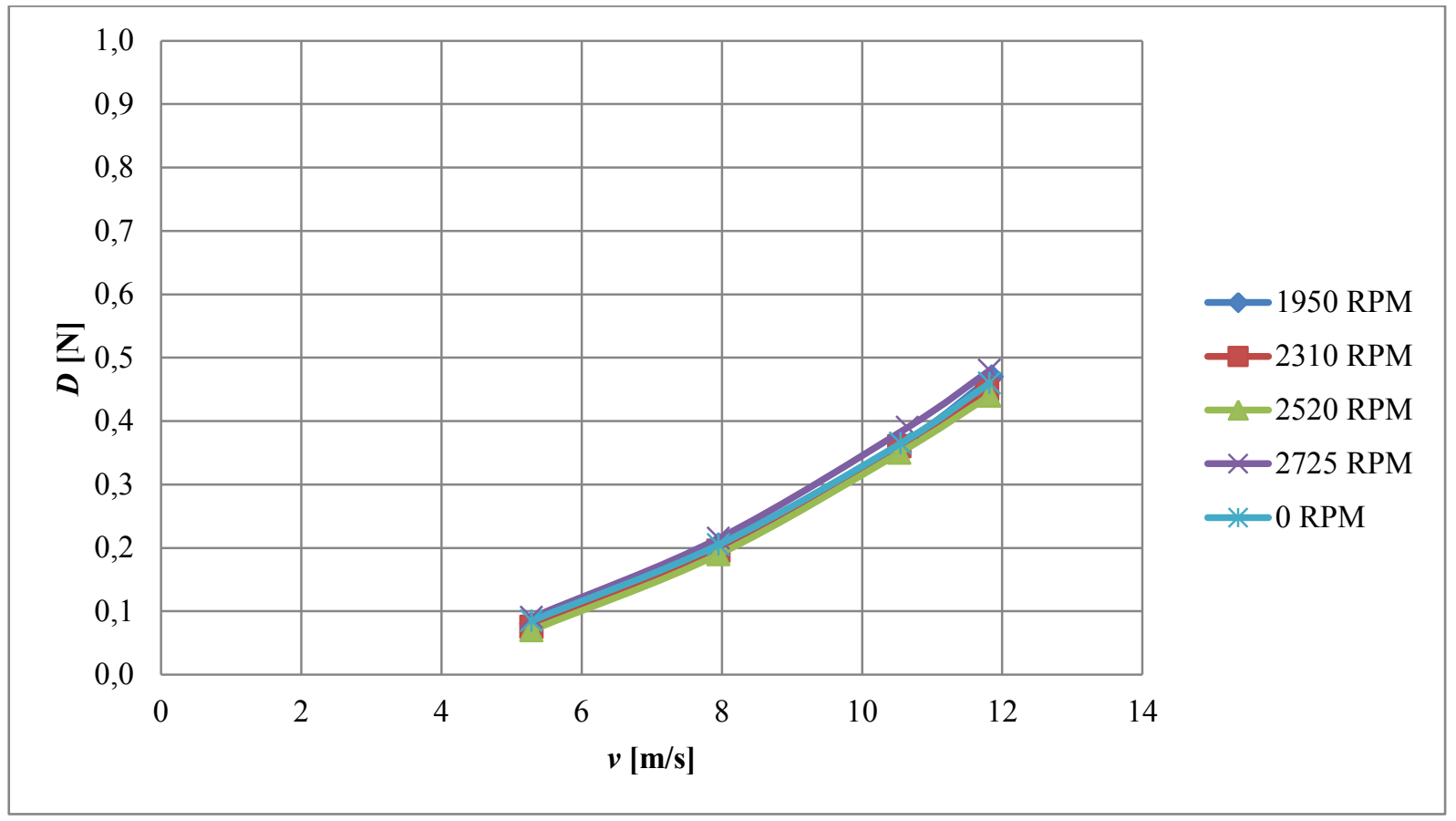

Figura A1.15 Variación de la resistencia $D$ en función de la velocidad de la corriente $v$ para cada velocidad de giro $\omega$.

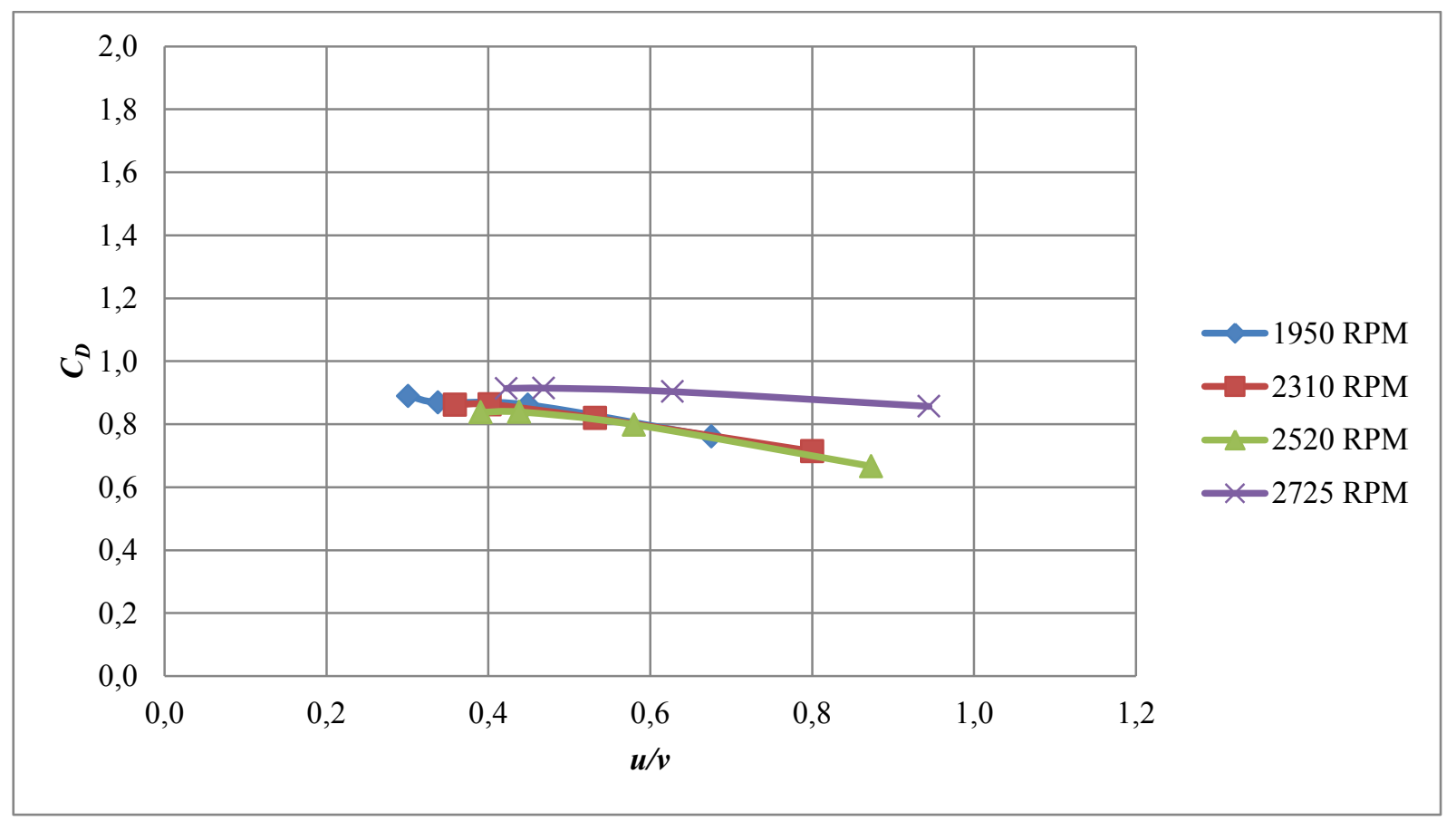

Figura A1.16 Variación del coeficiente de resistencia $C_{D}$ en función de la relación de velocidades $u / v$ para cada velocidad de giro $\omega$. 


\subsubsection{Circular con aletas}

Los resultados correspondientes a los ensayos de sustentación y resistencia se presentan en la tabla A1.6.

Tabla A1.6 Resultados correspondiente a los ensayos de sustentación y resistencia.

\begin{tabular}{|c|c|c|c|c|c|c|c|c|}
\hline \multicolumn{9}{|c|}{ Circular con aletas $(\phi=0,038 \mathrm{~m})$} \\
\hline $\begin{array}{c}\omega \\
{[\mathbf{r p m}]}\end{array}$ & $\begin{array}{c}v \\
{[\mathbf{m} / \mathbf{s}]}\end{array}$ & $\begin{array}{c}L \\
{[\mathbf{N}]}\end{array}$ & $C_{L}$ & $u / v$ & $\begin{array}{c}v \\
{[\mathbf{m} / \mathbf{s}]}\end{array}$ & $\begin{array}{c}D \\
{[\mathbf{N}]}\end{array}$ & $C_{D}$ & $u / v$ \\
\hline \multirow[t]{4}{*}{0} & - & - & - & - & 5,28 & 0,14 & 1,25 & 0,00 \\
\hline & - & - & - & - & 7,95 & 0,32 & 1,24 & 0,00 \\
\hline & - & - & - & - & 10,58 & 0,55 & 1,22 & 0,00 \\
\hline & - & - & - & - & 11,82 & 0,71 & 1,25 & 0,00 \\
\hline \multirow[t]{4}{*}{1950} & 5,22 & 0,18 & 1,58 & 0,74 & 5,32 & 0,12 & 1,05 & 0,73 \\
\hline & 7,88 & 0,23 & 0,91 & 0,49 & 7,95 & 0,28 & 1,10 & 0,49 \\
\hline & 10,51 & 0,33 & 0,74 & 0,37 & 10,52 & 0,61 & 1,36 & 0,37 \\
\hline & 11,77 & 0,40 & 0,71 & 0,33 & 11,80 & 0,78 & 1,39 & 0,33 \\
\hline \multirow[t]{4}{*}{2310} & 5,26 & 0,23 & 2,00 & 0,87 & 5,28 & 0,12 & 1,07 & 0,87 \\
\hline & 7,90 & 0,30 & 1,18 & 0,58 & 7,95 & 0,27 & 1,06 & 0,58 \\
\hline & 10,49 & 0,38 & 0,84 & 0,44 & 10,52 & 0,54 & 1,20 & 0,44 \\
\hline & 11,79 & 0,44 & 0,77 & 0,39 & 11,78 & 0,73 & 1,30 & 0,39 \\
\hline \multirow[t]{4}{*}{2520} & 5,26 & 0,26 & 2,27 & 0,95 & 5,28 & 0,13 & 1,11 & 0,95 \\
\hline & 7,90 & 0,36 & 1,40 & 0,63 & 7,92 & 0,27 & 1,05 & 0,63 \\
\hline & 10,49 & 0,42 & 0,94 & 0,48 & 10,54 & 0,52 & 1,15 & 0,48 \\
\hline & 11,79 & 0,47 & 0,82 & 0,43 & 11,78 & 0,69 & 1,23 & 0,43 \\
\hline \multirow[t]{4}{*}{2725} & 5,14 & 0,28 & 2,61 & 1,05 & 5,47 & 0,14 & 1,16 & 0,99 \\
\hline & 7,85 & 0,40 & 1,58 & 0,69 & 7,98 & 0,28 & 1,09 & 0,68 \\
\hline & 10,47 & 0,46 & 1,03 & 0,52 & 10,56 & 0,51 & 1,13 & 0,51 \\
\hline & 11,79 & 0,52 & 0,91 & 0,46 & 11,80 & 0,67 & 1,19 & 0,46 \\
\hline
\end{tabular}




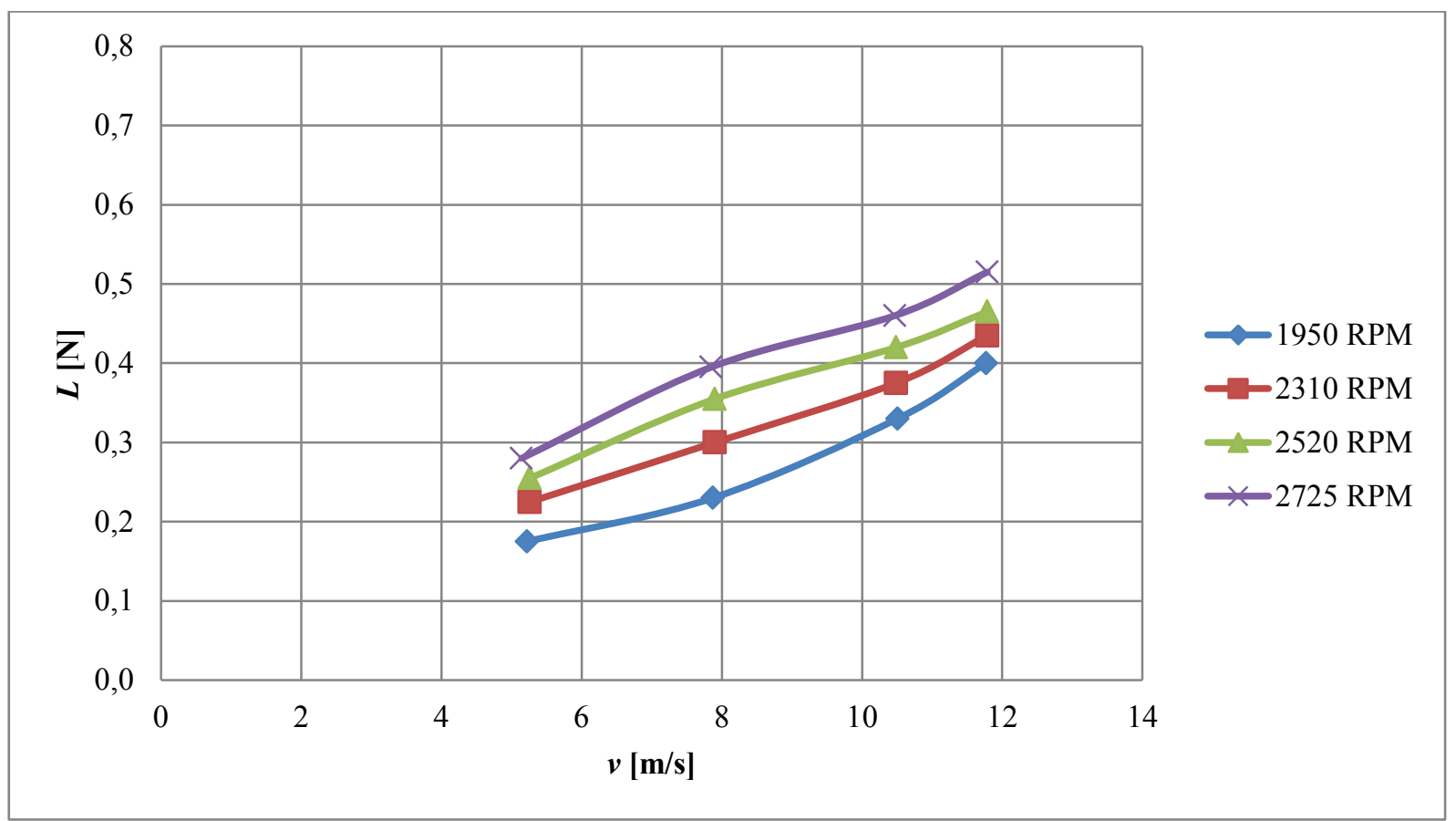

Figura A1.17 Variación de la sustentación $L$ en función de la velocidad de la corriente $v$ para cada velocidad de giro $\omega$.

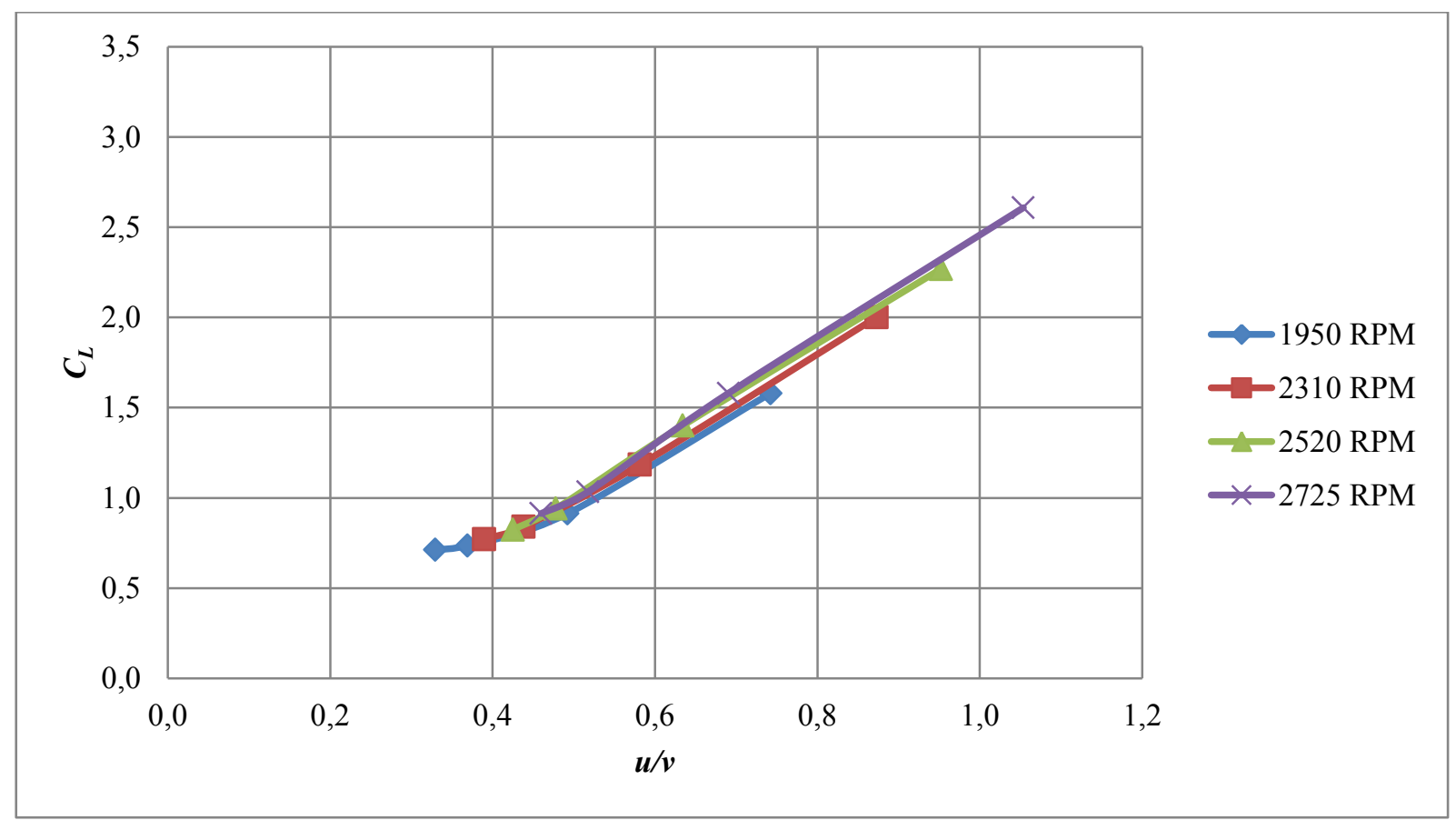

Figura A1.18 Variación del coeficiente de sustentación $C_{L}$ en función de la relación de velocidades $u / v$ para cada velocidad de giro $\omega$. 


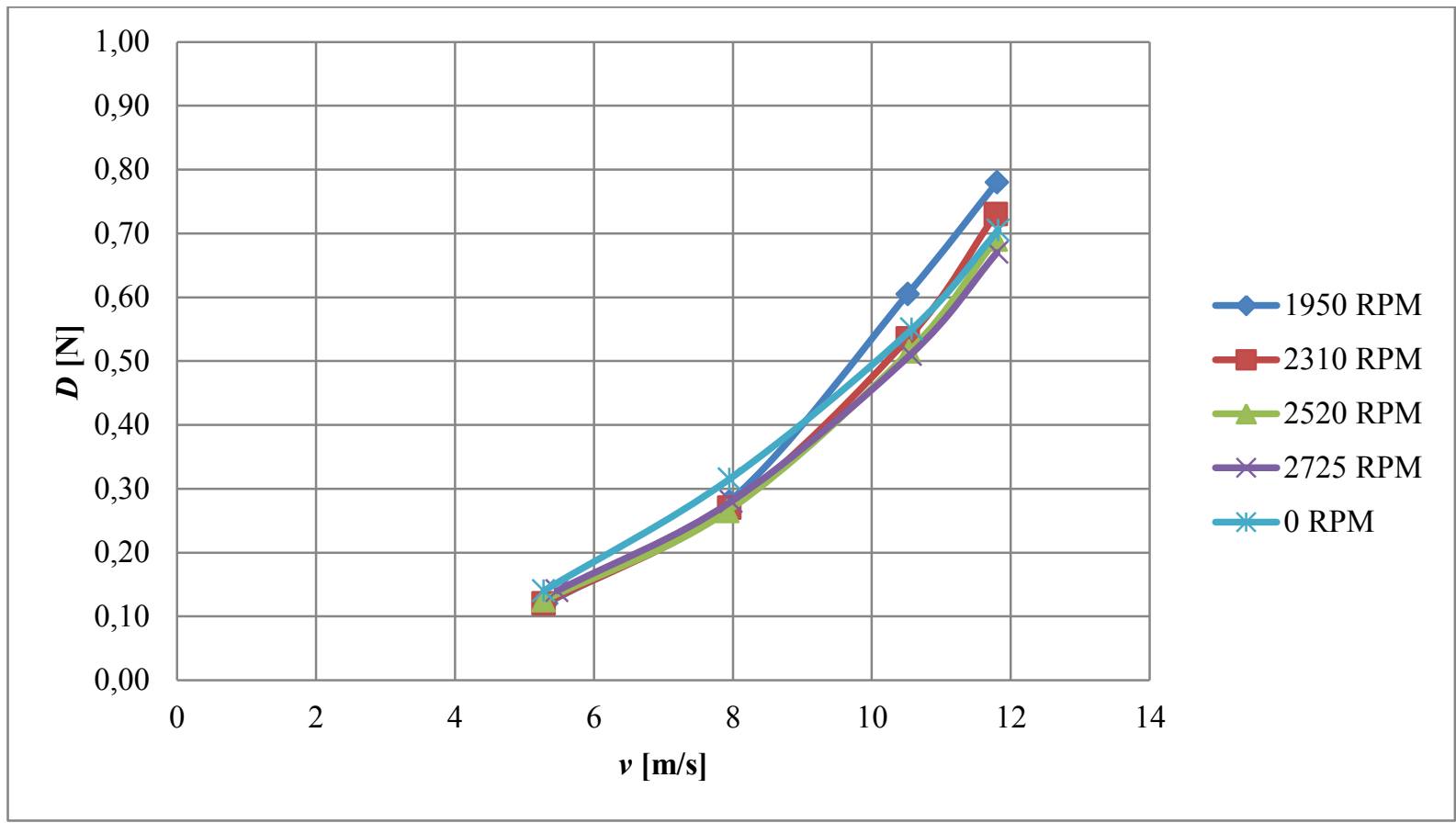

Figura A1.19 Variación de la resistencia $D$ en función de la velocidad de la corriente $v$ para cada velocidad de giro $\omega$.

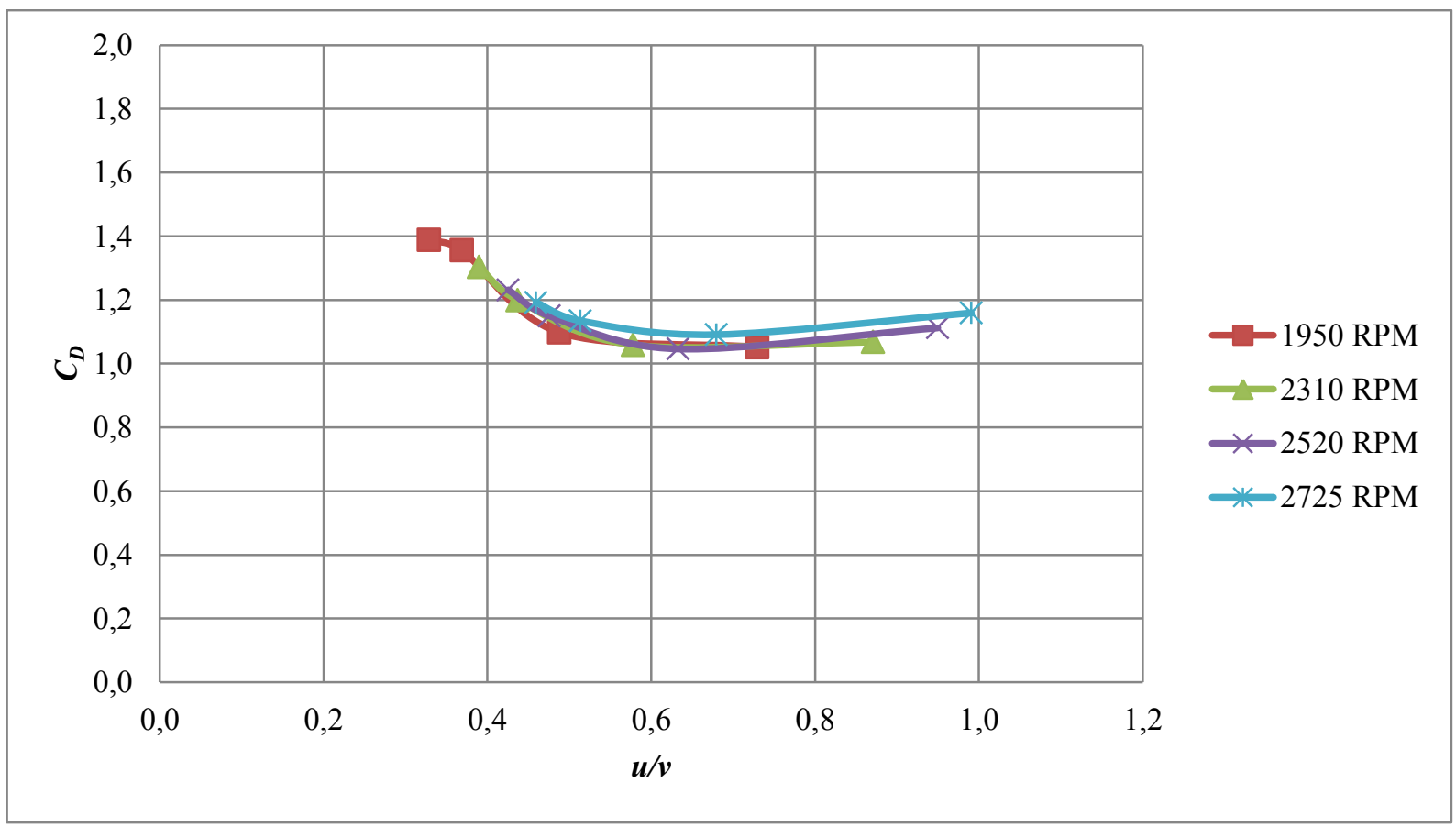

Figura A1.20 Variación del coeficiente de resistencia $C_{\boldsymbol{D}}$ en función de la relación de velocidades $u / v$ para cada velocidad de giro $\omega$. 


\subsubsection{Decágono}

Los resultados correspondientes a los ensayos de sustentación y resistencia se presentan en la tabla A1.7.

Tabla A1.7 Resultados correspondiente a los ensayos de sustentación y resistencia.

\begin{tabular}{|c|c|c|c|c|c|c|c|c|}
\hline \multicolumn{9}{|c|}{ Decágono $(\phi=0,036 \mathrm{~m})$} \\
\hline $\begin{array}{c}\omega \\
{[\mathrm{rpm}]}\end{array}$ & $\begin{array}{c}v \\
{[\mathrm{~m} / \mathrm{s}]}\end{array}$ & $\begin{array}{c}L \\
{[\mathbf{N}]}\end{array}$ & $C_{L}$ & $u / v$ & $\begin{array}{c}v \\
{[\mathrm{~m} / \mathrm{s}]}\end{array}$ & $\begin{array}{c}D \\
{[\mathbf{N}]}\end{array}$ & $C_{D}$ & $u / v$ \\
\hline \multirow[t]{4}{*}{0} & - & - & - & - & 5,27 & 0,11 & 1,02 & 0,00 \\
\hline & - & - & - & - & 7,82 & 0,24 & 1,01 & 0,00 \\
\hline & - & - & - & - & 10,30 & 0,42 & 1,00 & 0,00 \\
\hline & - & - & - & - & 11,43 & 0,52 & 1,02 & 0,00 \\
\hline \multirow[t]{4}{*}{1950} & 5,27 & 0,13 & 1,20 & 0,70 & 5,27 & 0,06 & 0,56 & 0,70 \\
\hline & 7,88 & 0,18 & 0,72 & 0,47 & 7,87 & 0,15 & 0,62 & 0,47 \\
\hline & 10,27 & 0,23 & 0,56 & 0,36 & 10,34 & 0,28 & 0,67 & 0,36 \\
\hline & 11,44 & 0,27 & 0,52 & 0,32 & 11,50 & 0,35 & 0,68 & 0,32 \\
\hline \multirow[t]{4}{*}{2310} & 5,27 & 0,17 & 1,53 & 0,83 & 5,27 & 0,06 & 0,56 & 0,83 \\
\hline & 7,88 & 0,22 & 0,91 & 0,55 & 7,87 & 0,14 & 0,58 & 0,55 \\
\hline & 10,27 & 0,26 & 0,63 & 0,42 & 10,30 & 0,26 & 0,63 & 0,42 \\
\hline & 11,40 & 0,29 & 0,57 & 0,38 & 11,46 & 0,34 & 0,66 & 0,38 \\
\hline \multirow[t]{4}{*}{2520} & 5,27 & 0,18 & 1,67 & 0,90 & 5,27 & 0,06 & 0,56 & 0,90 \\
\hline & 7,88 & 0,25 & 1,03 & 0,60 & 7,87 & 0,15 & 0,60 & 0,60 \\
\hline & 10,27 & 0,29 & 0,71 & 0,46 & 10,34 & 0,26 & 0,62 & 0,46 \\
\hline & 11,44 & 0,32 & 0,62 & 0,42 & 11,46 & 0,34 & 0,66 & 0,41 \\
\hline \multirow[t]{4}{*}{2725} & 5,27 & 0,20 & 1,85 & 0,97 & 5,27 & 0,06 & 0,56 & 0,98 \\
\hline & 7,88 & 0,28 & 1,16 & 0,65 & 7,87 & 0,15 & 0,60 & 0,65 \\
\hline & 10,27 & 0,33 & 0,79 & 0,50 & 10,30 & 0,26 & 0,63 & 0,50 \\
\hline & 11,44 & 0,35 & 0,69 & 0,45 & 11,43 & 0,34 & 0,66 & 0,45 \\
\hline
\end{tabular}




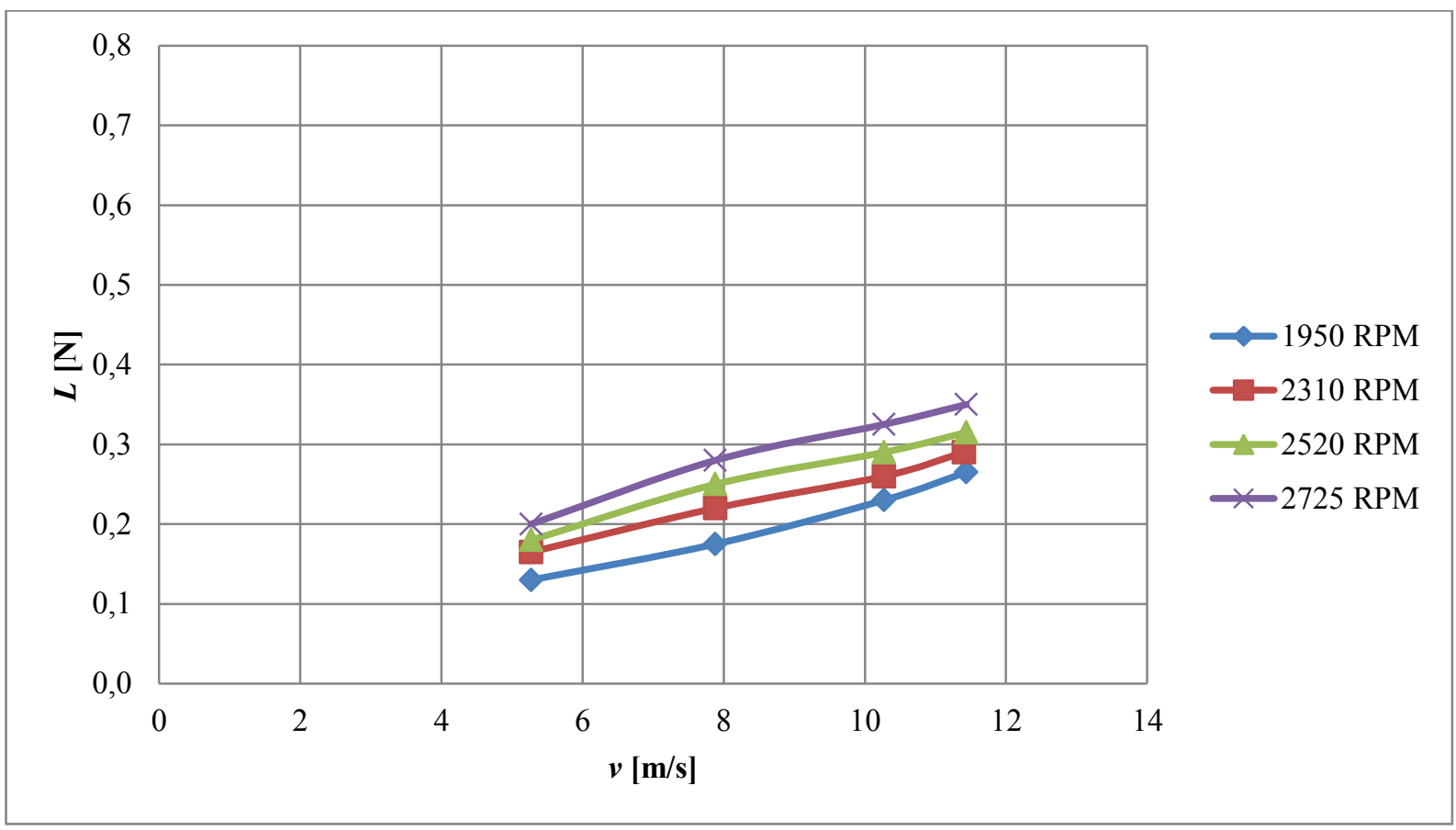

Figura A1.21 Variación de la sustentación $L$ en función de la velocidad de la corriente $v$ para cada velocidad de giro $\omega$.

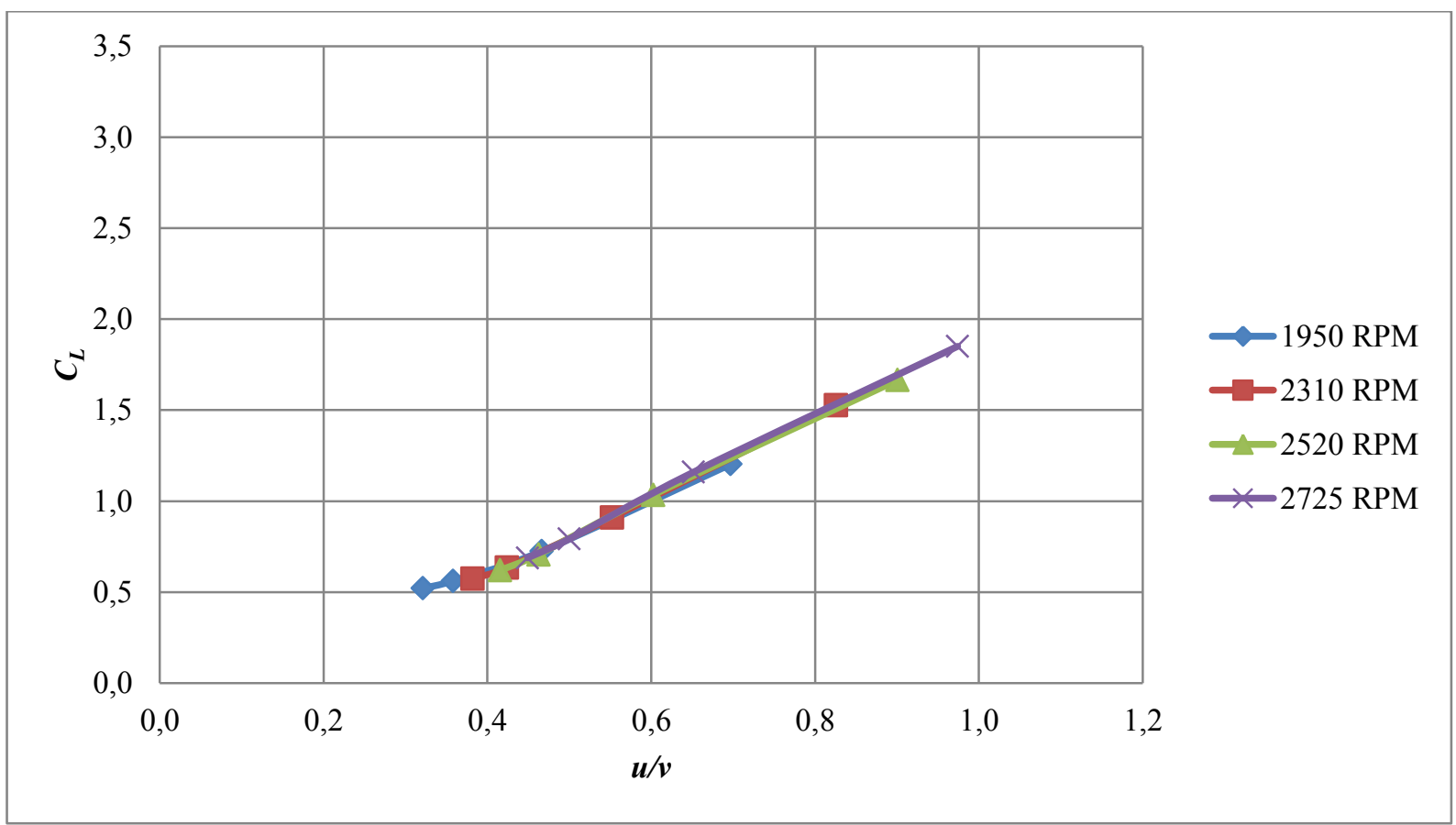

Figura A1.22 Variación del coeficiente de sustentación $C_{L}$ en función de la relación de velocidades $u / v$ para cada velocidad de giro $\omega$. 


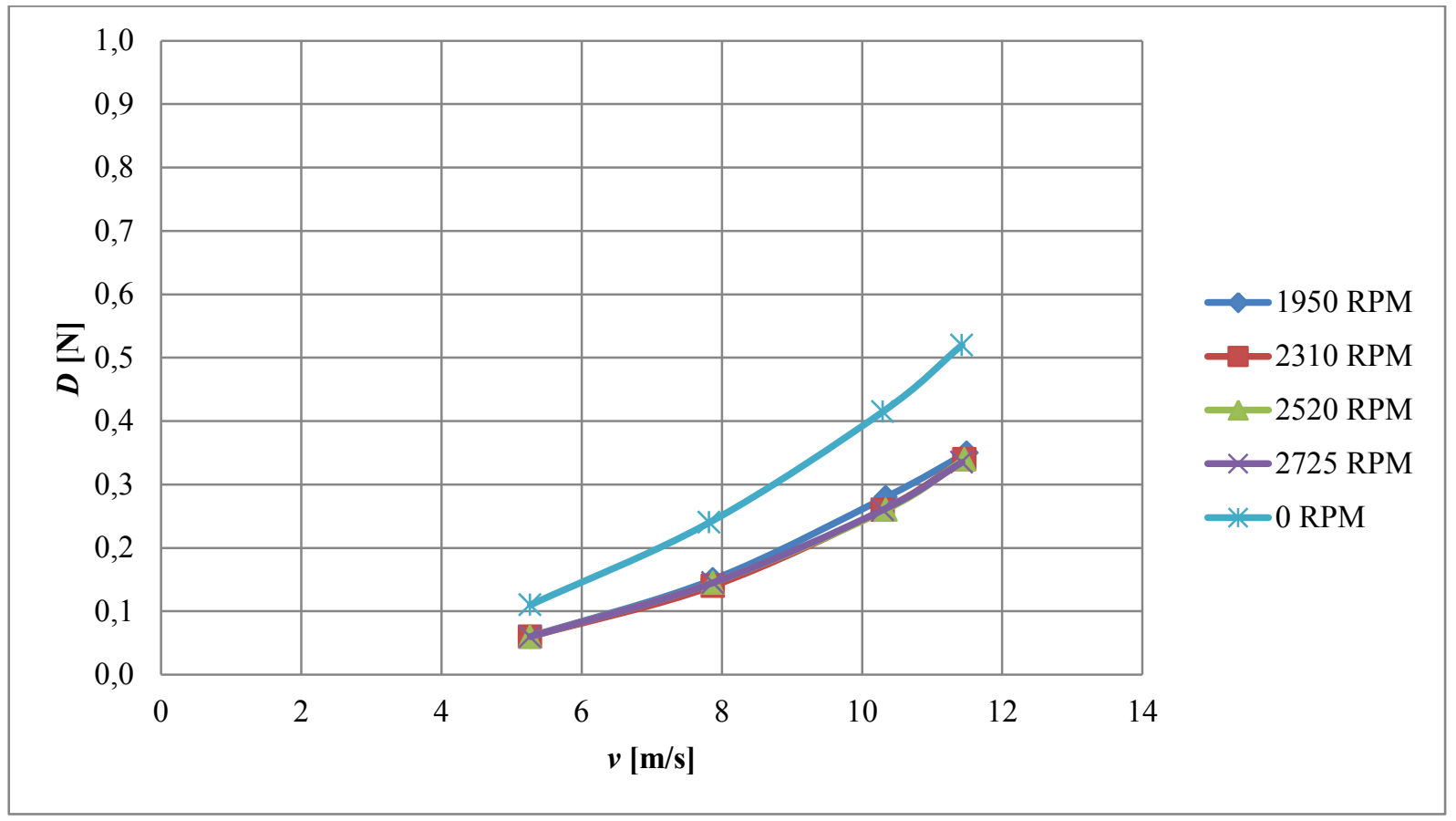

Figura A1.23 Variación de la resistencia $D$ en función de la velocidad de la corriente $v$ para cada velocidad de giro $\omega$.

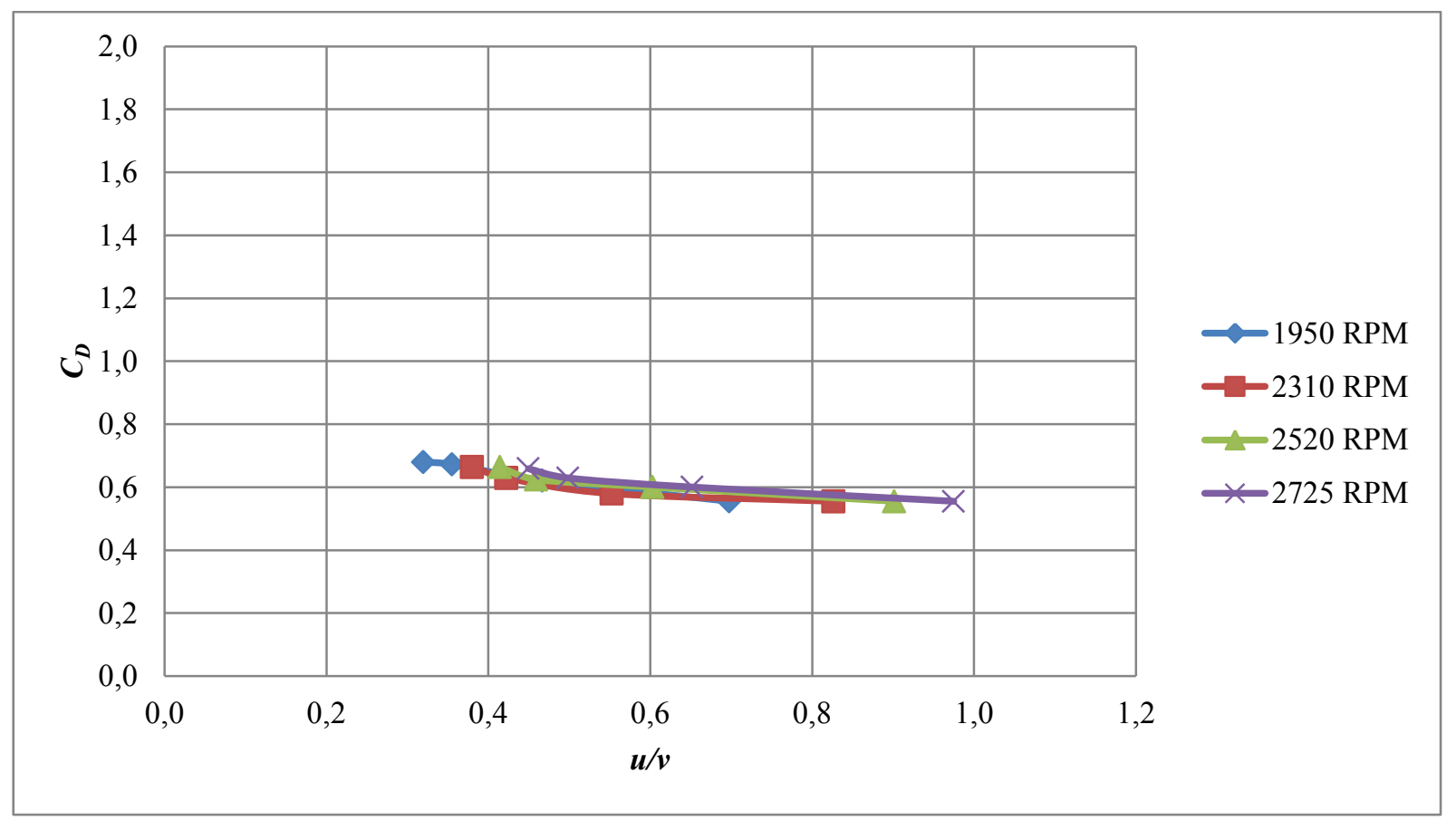

Figura A1.24 Variación del coeficiente de resistencia $C_{\boldsymbol{D}}$ en función de la relación de velocidades $u / v$ para cada velocidad de giro $\omega$. 


\subsubsection{Octógono}

Los resultados correspondientes a los ensayos de sustentación y resistencia se presentan en la tabla A1.8.

Tabla A1.8 Resultados correspondiente a los ensayos de sustentación y resistencia.

\begin{tabular}{|c|c|c|c|c|c|c|c|c|}
\hline \multicolumn{9}{|c|}{ Octógono $(\phi=0,0365 \mathrm{~m})$} \\
\hline $\begin{array}{c}\omega \\
{[\mathbf{r p m}]}\end{array}$ & $\begin{array}{c}v \\
{[\mathbf{m} / \mathbf{s}]}\end{array}$ & $\begin{array}{c}\boldsymbol{L} \\
{[\mathbf{N}]}\end{array}$ & $C_{L}$ & $u / v$ & $\begin{array}{c}v \\
{[\mathbf{m} / \mathbf{s}]}\end{array}$ & $\begin{array}{c}D \\
{[\mathbf{N}]}\end{array}$ & $C_{D}$ & $u / v$ \\
\hline \multirow[t]{4}{*}{0} & - & - & - & - & 5,20 & 0,12 & 1,13 & 0,00 \\
\hline & - & - & - & - & 7,84 & 0,28 & 1,14 & 0,00 \\
\hline & - & - & - & - & 10,32 & 0,50 & 1,18 & 0,00 \\
\hline & - & - & - & - & 11,41 & 0,62 & 1,20 & 0,00 \\
\hline \multirow[t]{4}{*}{1950} & 5,27 & 0,15 & 1,32 & 0,71 & 5,28 & 0,07 & 0,64 & 0,71 \\
\hline & 7,83 & 0,19 & 0,79 & 0,48 & 7,94 & 0,17 & 0,66 & 0,47 \\
\hline & 10,27 & 0,23 & 0,54 & 0,36 & 10,36 & 0,30 & 0,71 & 0,36 \\
\hline & 11,40 & 0,26 & 0,50 & 0,33 & 11,48 & 0,38 & 0,73 & 0,32 \\
\hline \multirow[t]{4}{*}{2310} & 5,27 & 0,16 & 1,46 & 0,84 & 5,28 & 0,07 & 0,64 & 0,84 \\
\hline & 7,83 & 0,23 & 0,93 & 0,56 & 7,94 & 0,17 & 0,66 & 0,56 \\
\hline & 10,27 & 0,28 & 0,66 & 0,43 & 10,36 & 0,30 & 0,71 & 0,43 \\
\hline & 11,40 & 0,29 & 0,57 & 0,39 & 11,48 & 0,38 & 0,73 & 0,38 \\
\hline \multirow[t]{4}{*}{2520} & 5,23 & 0,19 & 1,76 & 0,92 & 5,28 & 0,07 & 0,64 & 0,91 \\
\hline & 7,83 & 0,27 & 1,10 & 0,61 & 7,94 & 0,16 & 0,64 & 0,61 \\
\hline & 10,23 & 0,31 & 0,74 & 0,47 & 10,36 & 0,30 & 0,70 & 0,46 \\
\hline & 11,37 & 0,34 & 0,66 & 0,42 & 11,55 & 0,38 & 0,71 & 0,42 \\
\hline \multirow[t]{4}{*}{2725} & 5,19 & 0,20 & 1,88 & 1,00 & 5,28 & 0,08 & 0,68 & 0,99 \\
\hline & 7,83 & 0,29 & 1,18 & 0,66 & 7,94 & 0,17 & 0,69 & 0,66 \\
\hline & 10,23 & 0,33 & 0,80 & 0,51 & 10,36 & 0,29 & 0,69 & 0,50 \\
\hline & 11,37 & 0,37 & 0,72 & 0,46 & 11,52 & 0,37 & 0,71 & 0,45 \\
\hline
\end{tabular}




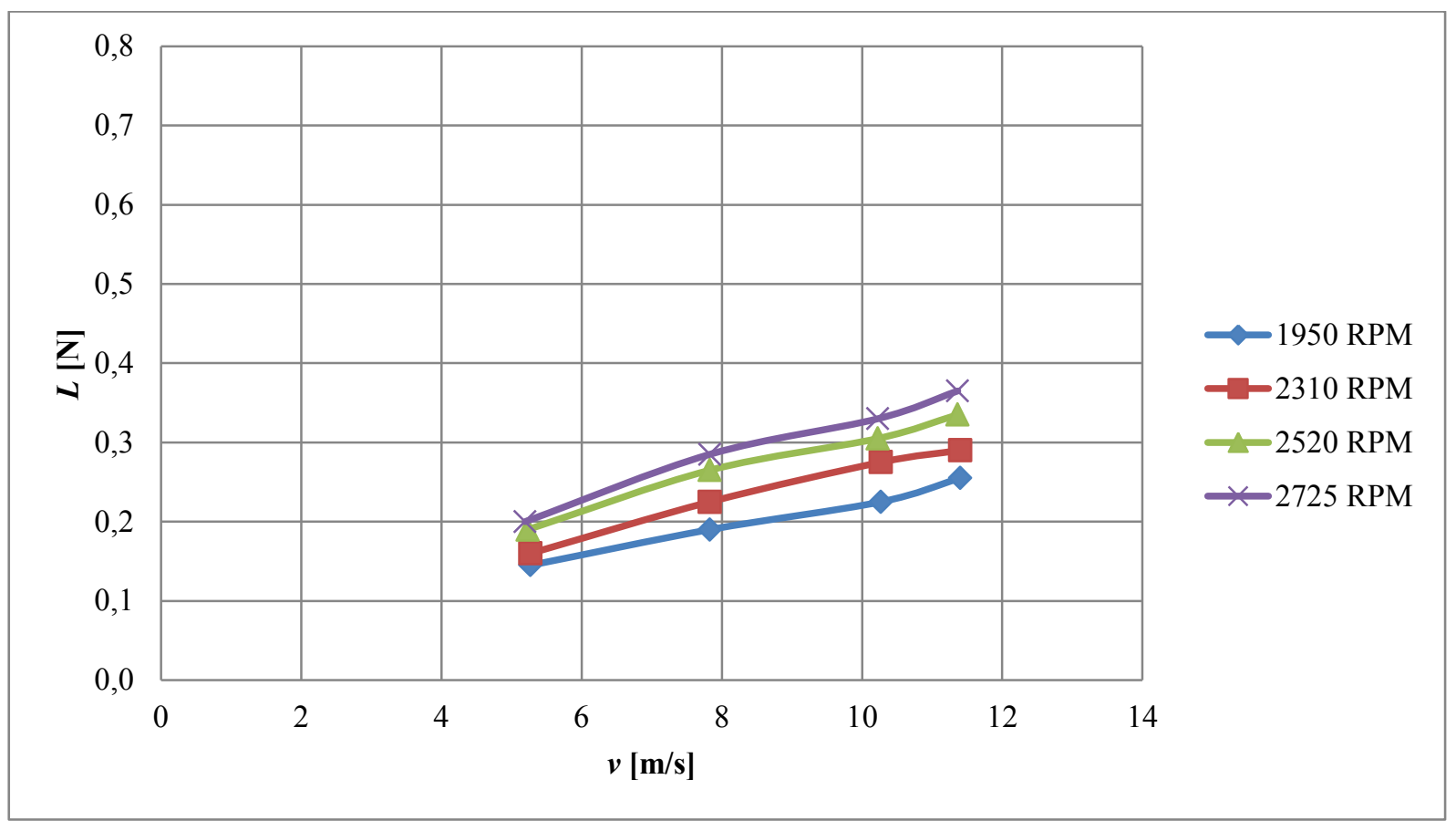

Figura A1.25 Variación de la sustentación $L$ en función de la velocidad de la corriente $v$ para cada velocidad de giro $\omega$.

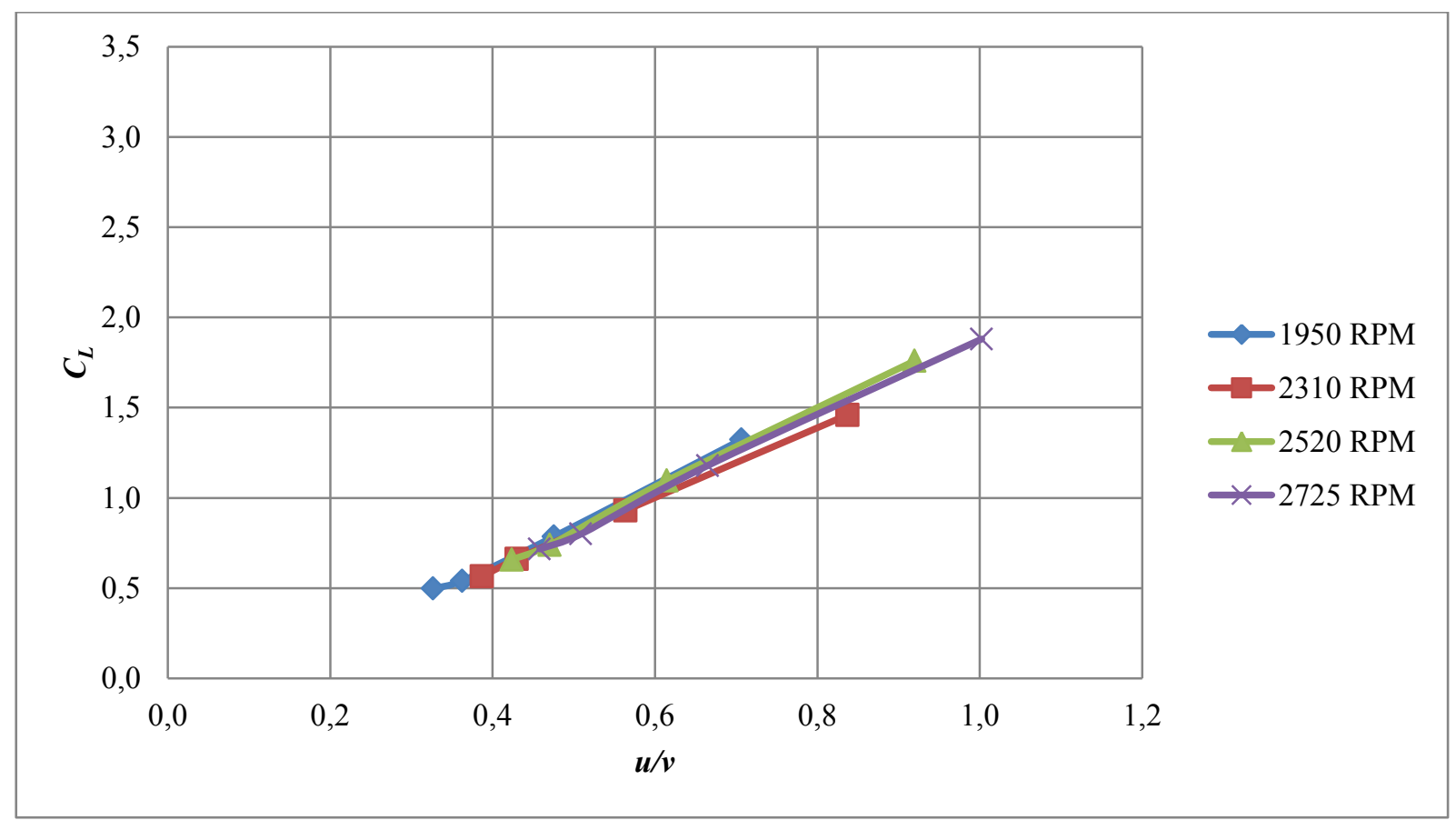

Figura A1.26 Variación del coeficiente de sustentación $C_{L}$ en función de la relación de velocidades $u / v$ para cada velocidad de giro $\omega$. 


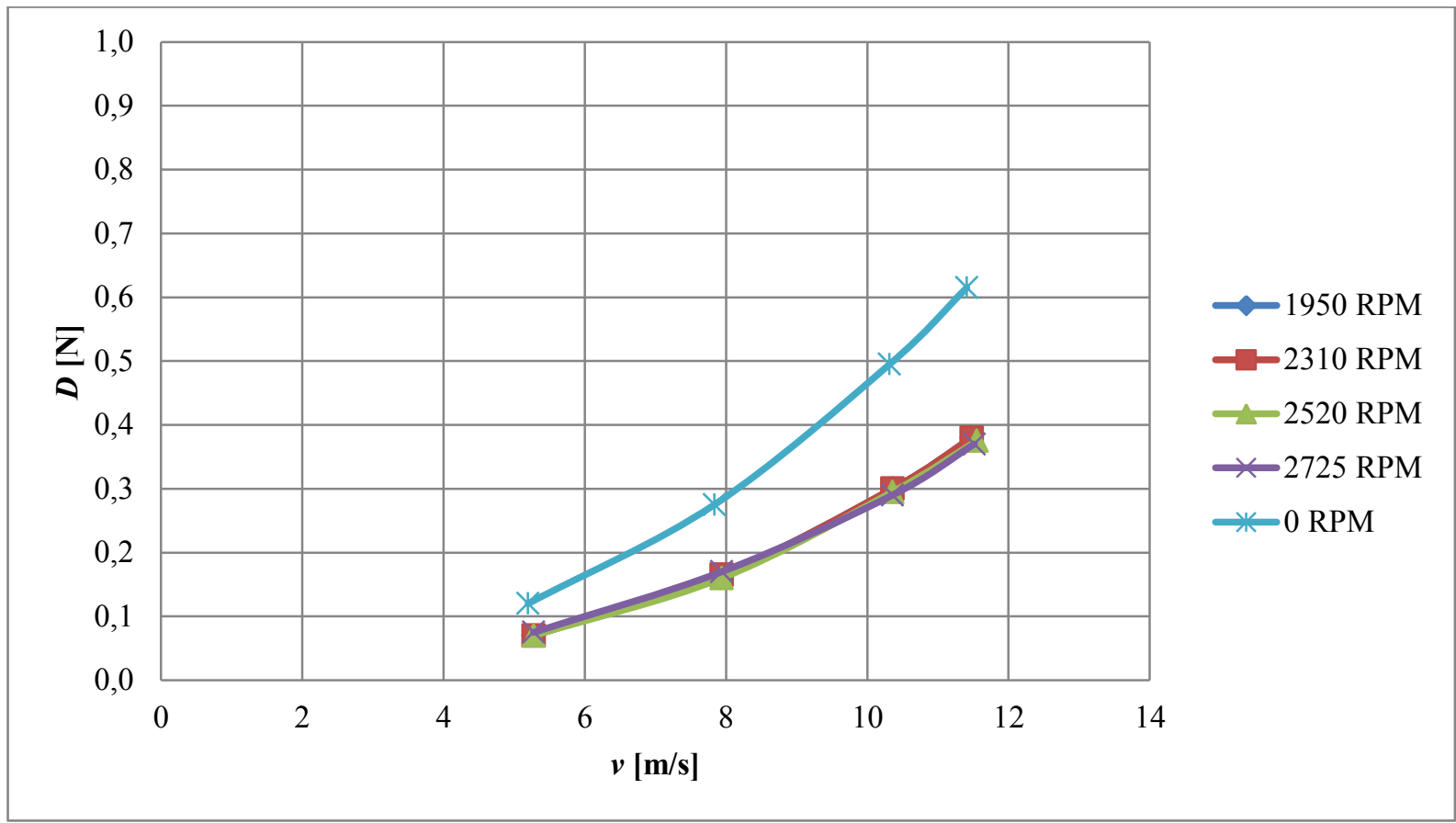

Figura A1.27 Variación de la resistencia $D$ en función de la velocidad de la corriente $v$ para cada velocidad de giro $\omega$.

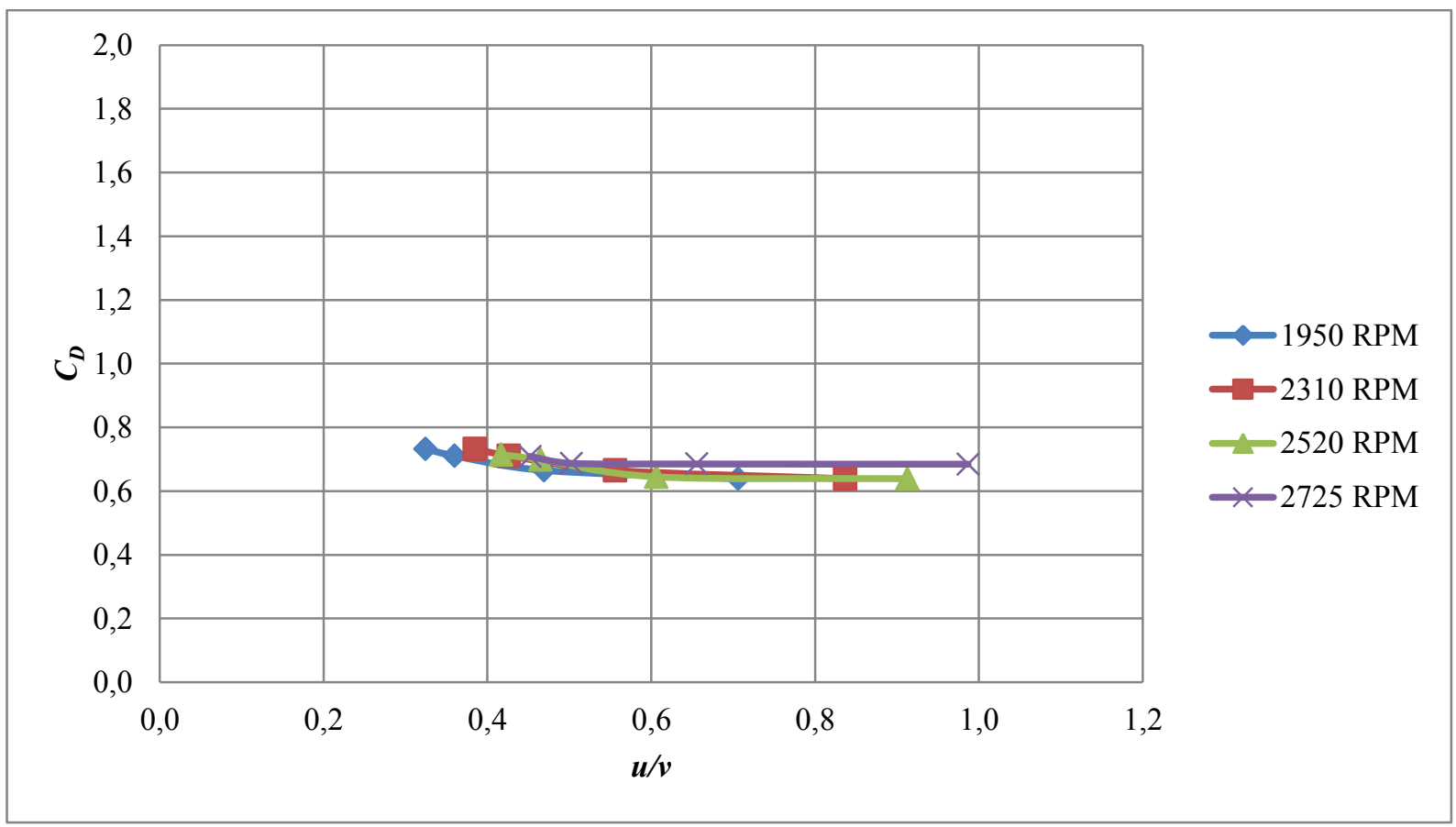

Figura A1.28 Variación del coeficiente de resistencia $C_{\boldsymbol{D}}$ en función de la relación de velocidades $u / v$ para cada velocidad de giro $\omega$. 


\subsubsection{Hexágono}

Los resultados correspondientes a los ensayos de sustentación y resistencia se presentan en la tabla A1.9.

Tabla A1.9 Resultados correspondiente a los ensayos de sustentación y resistencia.

\begin{tabular}{|c|c|c|c|c|c|c|c|c|}
\hline \multicolumn{9}{|c|}{ Hexágono $(\phi=0,0375 \mathrm{~m})$} \\
\hline $\begin{array}{c}\omega \\
{[\text { rpm }]}\end{array}$ & $\begin{array}{c}v \\
{[\mathrm{~m} / \mathrm{s}]}\end{array}$ & $\begin{array}{c}L \\
{[\mathbf{N}]}\end{array}$ & $C_{L}$ & $u / v$ & $\begin{array}{c}v \\
{[\mathrm{~m} / \mathrm{s}]}\end{array}$ & $\begin{array}{c}D \\
{[\mathbf{N}]}\end{array}$ & $C_{D}$ & $u / v$ \\
\hline \multirow[t]{4}{*}{0} & & - & - & . & 5,28 & 0,12 & 1,07 & 0,00 \\
\hline & - & - & - & - & 7,90 & 0,27 & 1,07 & 0,00 \\
\hline & - & - & - & - & 10,33 & 0,46 & 1,07 & 00 \\
\hline & - & - & - & - & 11,49 & 0,58 & 1,09 & 0,00 \\
\hline \multirow[t]{4}{*}{1950} & 5,27 & 0,15 & 1,33 & 0,73 & 5,28 & 0,09 & 0,80 & 0,72 \\
\hline & 7,83 & 0,19 & 0,77 & 0,49 & 7,95 & 0,21 & 0,80 & 0,48 \\
\hline & 10,19 & 0,23 & 0,55 & 0,38 & 10,37 & 0,39 & 0,89 & 0,37 \\
\hline & 11,26 & 0,25 & 0,49 & 0,34 & 11,49 & 0,50 & 0,93 & 0,33 \\
\hline \multirow[t]{4}{*}{2310} & 5,27 & 0,19 & 1,64 & 0,86 & 5,28 & 0,09 & 0,76 & 0,86 \\
\hline & 7,78 & 0,25 & 1,00 & 0,58 & 7,95 & 0,20 & 0,76 & 0,57 \\
\hline & 10,19 & 0,28 & 0,67 & 0,4 & 10,37 & 0,36 & 0,83 & 0,44 \\
\hline & 11,37 & 0,31 & 0,58 & 0,40 & 11,49 & 0,46 & 0,86 & 0,39 \\
\hline \multirow{4}{*}{2520} & 5,27 & 0,21 & 1,87 & 0,94 & 5,28 & 0,09 & 0,76 & 0,94 \\
\hline & 7,78 & 0,28 & 1,14 & 0,64 & 7,95 & 0,19 & 0,75 & 0,62 \\
\hline & 10,15 & 0,33 & 0,78 & 0,49 & 10,37 & 0,34 & 0,78 & 0,48 \\
\hline & 11,29 & 0,34 & 0,66 & 0,44 & 11,49 & 0,45 & 0,84 & 0,43 \\
\hline \multirow[t]{4}{*}{2725} & 5,27 & 0,24 & 2,09 & 1,01 & 5,28 & 0,09 & 0,77 & 1,01 \\
\hline & 7,78 & 0,32 & 1,29 & 0,69 & 7,95 & 0,19 & 0,75 & 0,67 \\
\hline & 10,15 & 0,37 & 0,87 & 0,5 & 10,37 & 0,35 & 0,80 & 0,52 \\
\hline & 11,33 & 0,39 & 0,74 & 0,47 & 11,49 & 0,45 & 0,83 & 0,47 \\
\hline
\end{tabular}




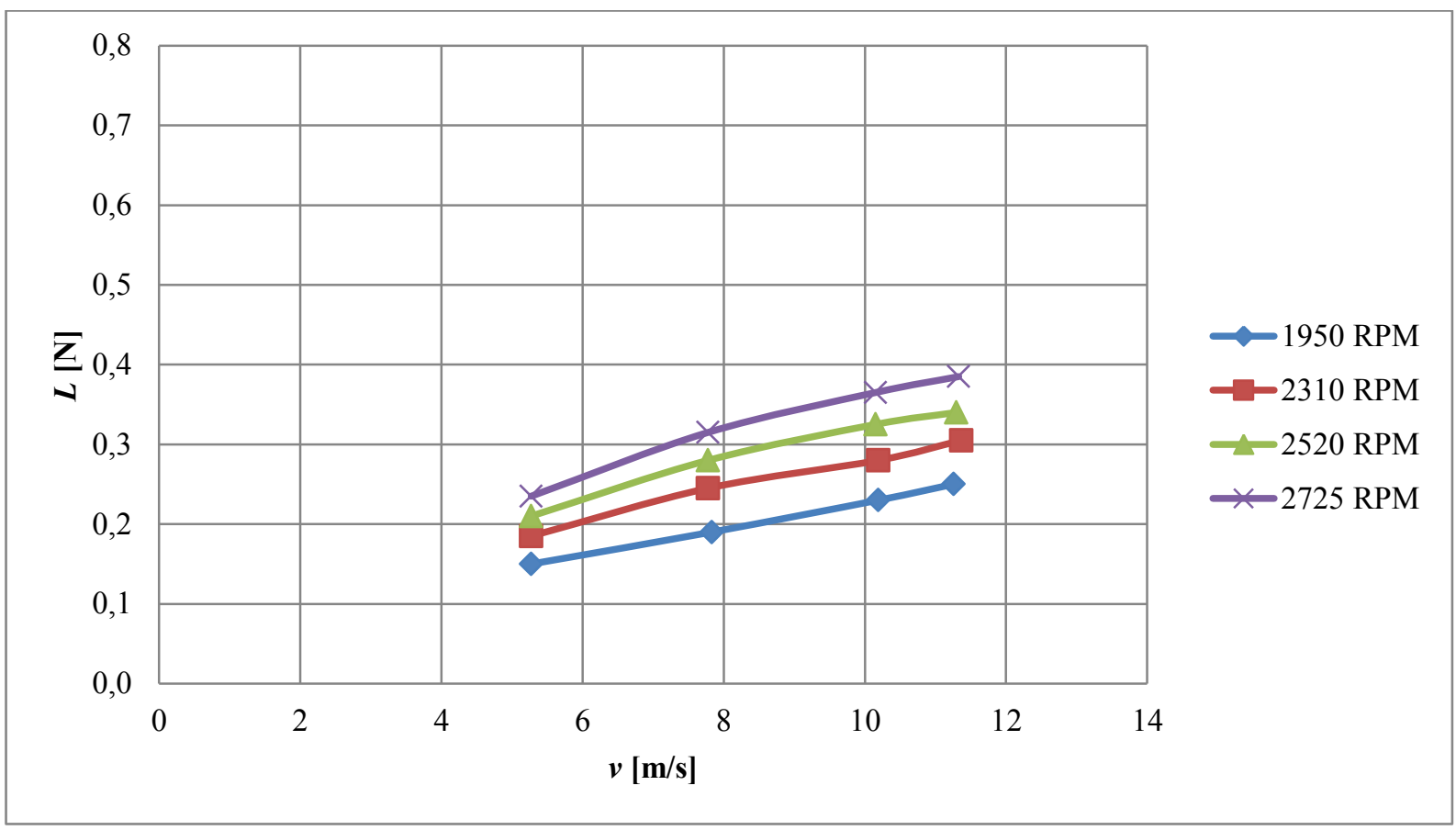

Figura A1.29 Variación de la sustentación $L$ en función de la velocidad de la corriente $v$ para cada velocidad de giro $\omega$.

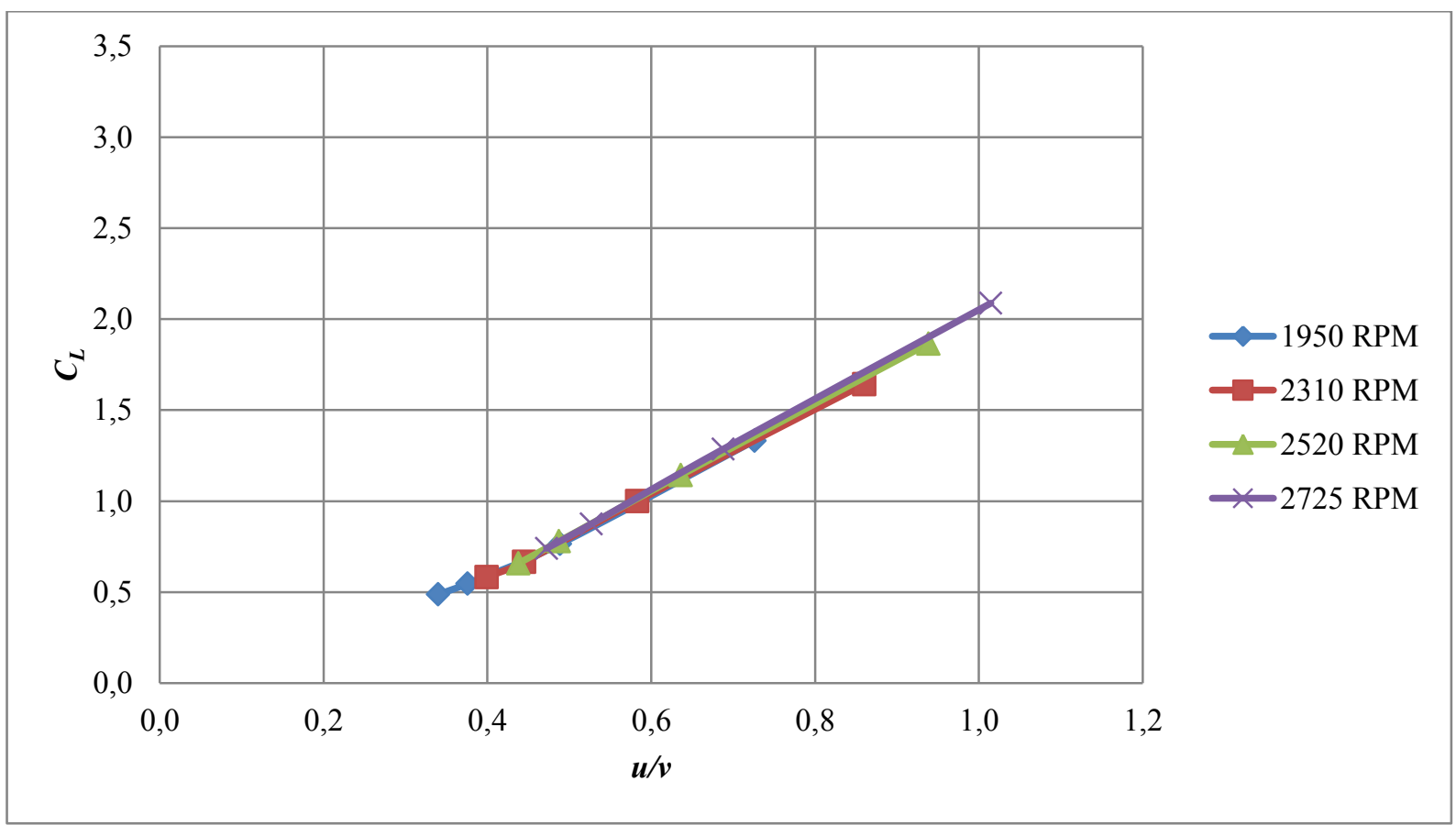

Figura A1.30 Variación del coeficiente de sustentación $C_{L}$ en función de la relación de velocidades $u / v$ para cada velocidad de giro $\omega$. 


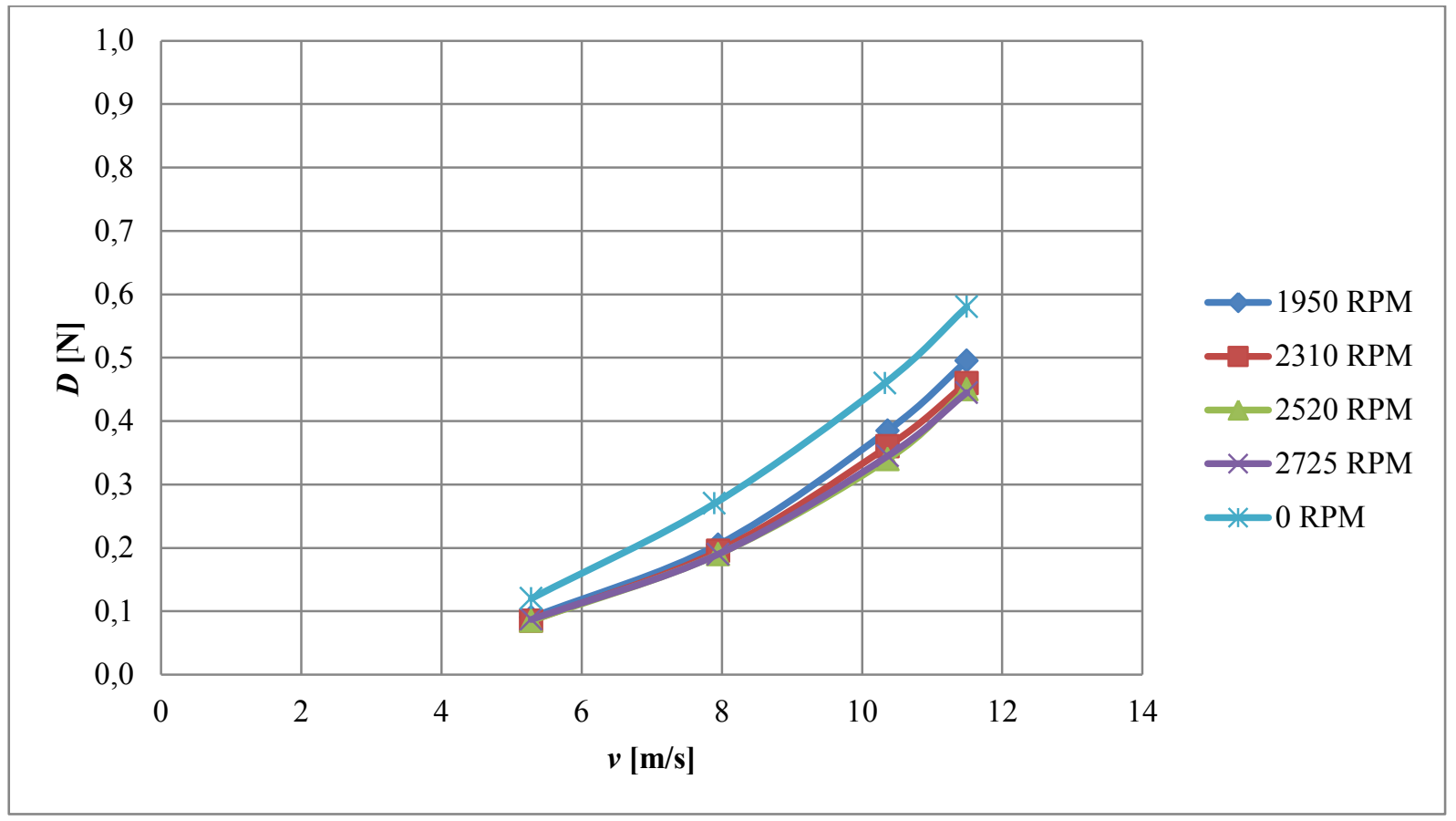

Figura A1.31 Variación de la resistencia $D$ en función de la velocidad de la corriente $v$ para cada velocidad de giro $\omega$.

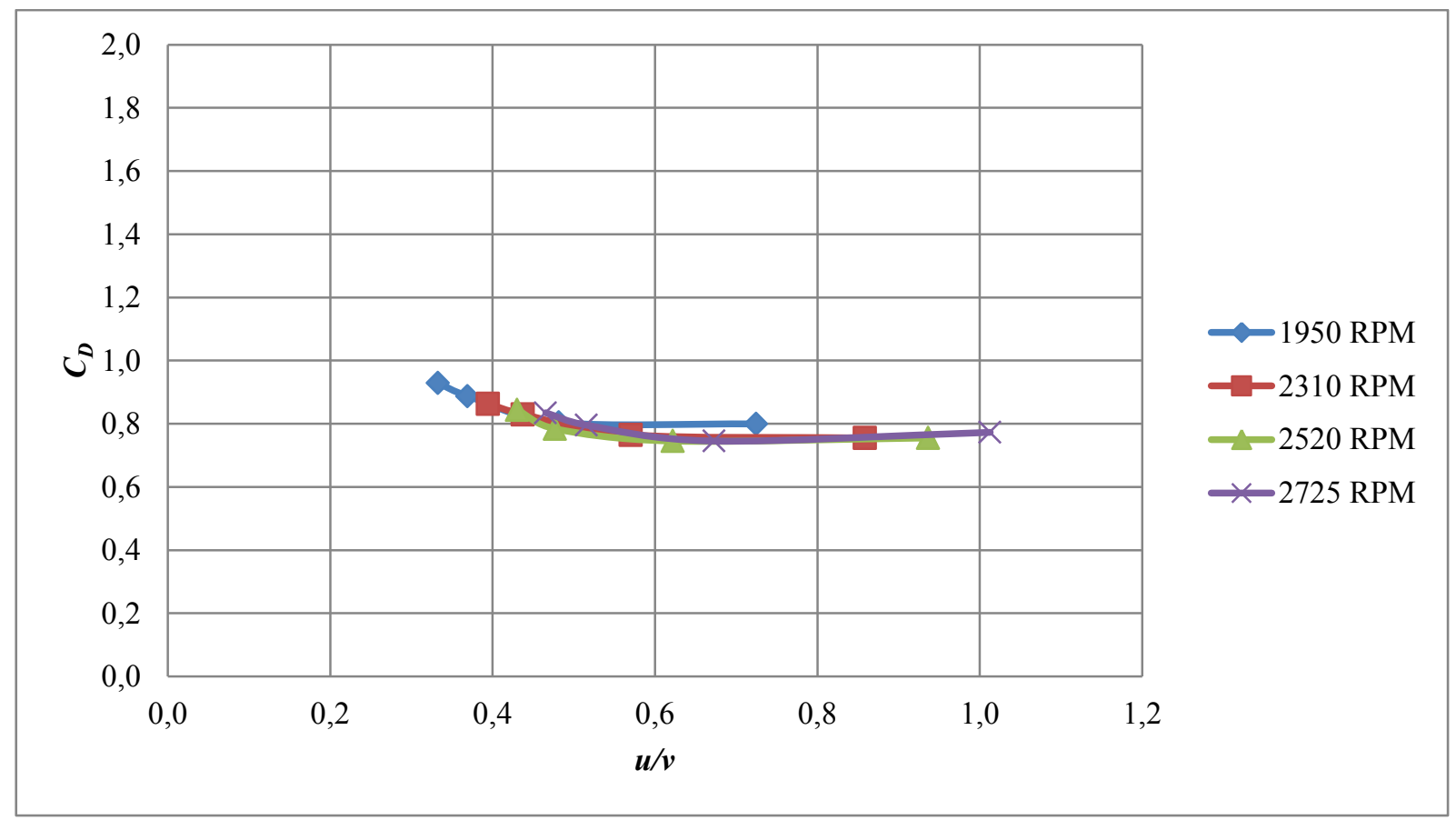

Figura A1.32 Variación del coeficiente de resistencia $C_{\boldsymbol{D}}$ en función de la relación de velocidades $u / v$ para cada velocidad de giro $\omega$. 


\subsubsection{Cuadrado}

Los resultados correspondientes a los ensayos de sustentación y resistencia se presentan en la tabla A1.10.

Tabla A1.10 Resultados correspondiente a los ensayos de sustentación y resistencia.

\begin{tabular}{|c|c|c|c|c|c|c|c|c|}
\hline \multicolumn{9}{|c|}{ Cuadrado $(\phi=0,042 \mathrm{~m})$} \\
\hline $\begin{array}{c}\omega \\
{[\mathrm{rpm}]}\end{array}$ & $\begin{array}{c}v \\
{[\mathbf{m} / \mathbf{s}]}\end{array}$ & $\begin{array}{c}L \\
{[\mathbf{N}]}\end{array}$ & $C_{L}$ & $u / v$ & $\begin{array}{c}v \\
{[\mathrm{~m} / \mathrm{s}]}\end{array}$ & $\begin{array}{c}D \\
{[\mathbf{N}]}\end{array}$ & $C_{D}$ & $u / v$ \\
\hline \multirow[t]{4}{*}{0} & - & - & - & - & 5,28 & 0,18 & 1,39 & 0,00 \\
\hline & - & - & - & - & 7,85 & 0,39 & 1,40 & 0,00 \\
\hline & - & - & - & - & 10,29 & 0,67 & 1,40 & 0,00 \\
\hline & - & - & - & - & 11,42 & 0,83 & 1,41 & 0,00 \\
\hline \multirow[t]{4}{*}{1950} & 5,28 & 0,23 & 1,82 & 0,81 & 5,28 & 0,15 & 1,19 & 0,81 \\
\hline & 7,84 & 0,30 & 1,08 & 0,55 & 7,89 & 0,32 & 1,14 & 0,54 \\
\hline & 10,16 & 0,39 & 0,83 & 0,42 & 10,28 & 0,57 & 1,19 & 0,42 \\
\hline & 11,38 & 0,44 & 0,75 & 0,38 & 11,41 & 0,72 & 1,22 & 0,38 \\
\hline \multirow[t]{4}{*}{2310} & 5,28 & 0,28 & 2,18 & 0,96 & 5,20 & 0,15 & 1,23 & 0,98 \\
\hline & 7,89 & 0,37 & 1,30 & 0,64 & 7,85 & 0,31 & 1,11 & 0,65 \\
\hline & 10,20 & 0,45 & 0,96 & 0,50 & 10,29 & 0,56 & 1,17 & 0,49 \\
\hline & 11,34 & 0,49 & 0,84 & 0,45 & 11,42 & 0,72 & 1,22 & 0,44 \\
\hline \multirow[t]{4}{*}{2520} & 5,28 & 0,32 & 2,54 & 1,05 & 5,20 & 0,16 & 1,31 & 1,06 \\
\hline & 7,84 & 0,42 & 1,51 & 0,71 & 7,85 & 0,31 & 1,11 & 0,71 \\
\hline & 10,16 & 0,51 & 1,08 & 0,55 & 10,29 & 0,56 & 1,17 & 0,54 \\
\hline & 11,30 & 0,55 & 0,94 & 0,49 & 11,42 & 0,71 & 1,20 & 0,49 \\
\hline \multirow[t]{4}{*}{2725} & 5,28 & 0,35 & 2,78 & 1,14 & 5,20 & 0,17 & 1,35 & 1,15 \\
\hline & 7,84 & 0,46 & 1,65 & 0,76 & 7,85 & 0,31 & 1,11 & 0,76 \\
\hline & 10,16 & 0,55 & 1,18 & 0,59 & 10,29 & 0,54 & 1,13 & 0,58 \\
\hline & 11,30 & 0,60 & 1,04 & 0,53 & 11,42 & 0,69 & 1,17 & 0,52 \\
\hline
\end{tabular}




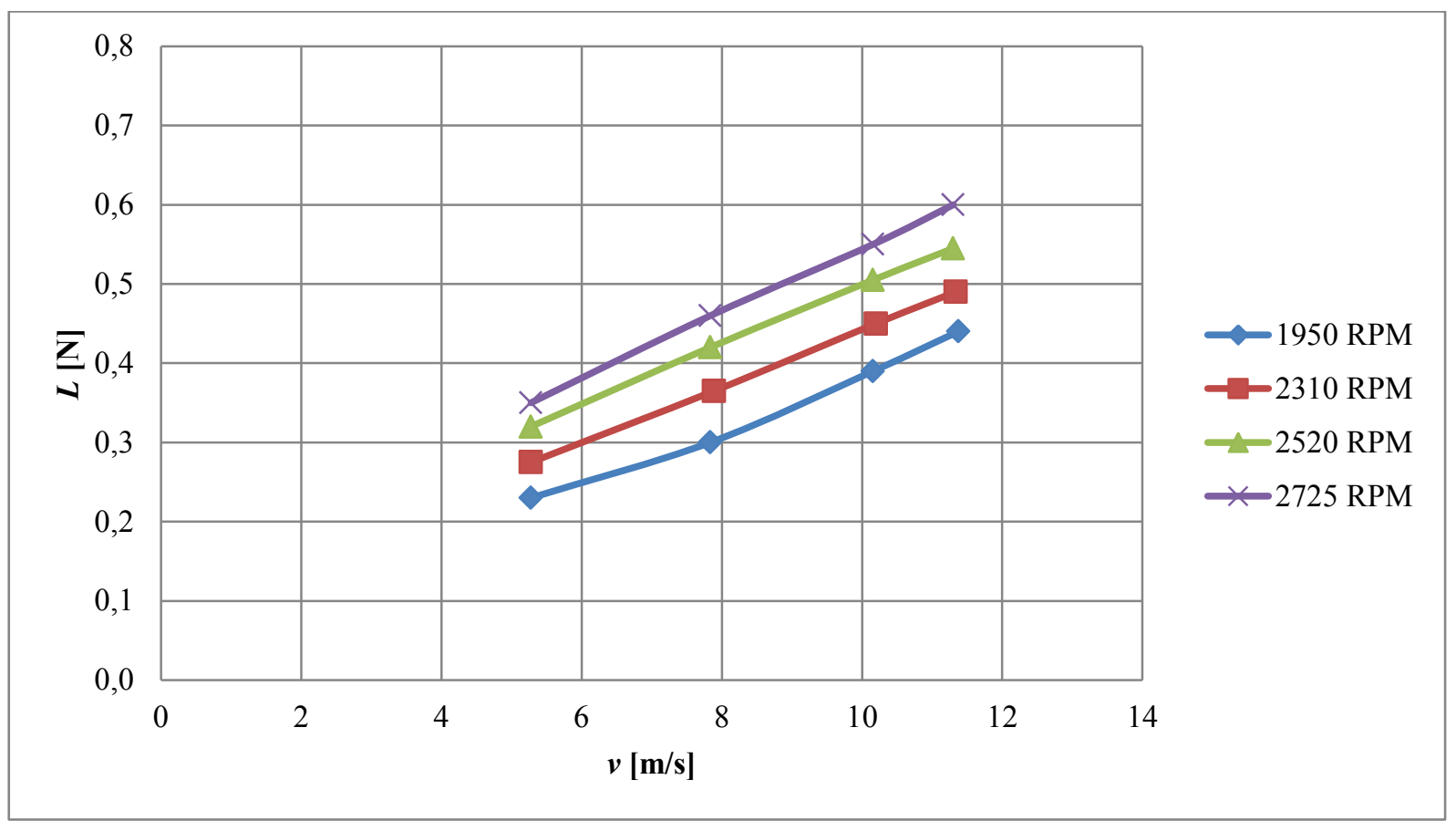

Figura A1.33 Variación de la sustentación $L$ en función de la velocidad de la corriente $v$ para cada velocidad de giro $\omega$.

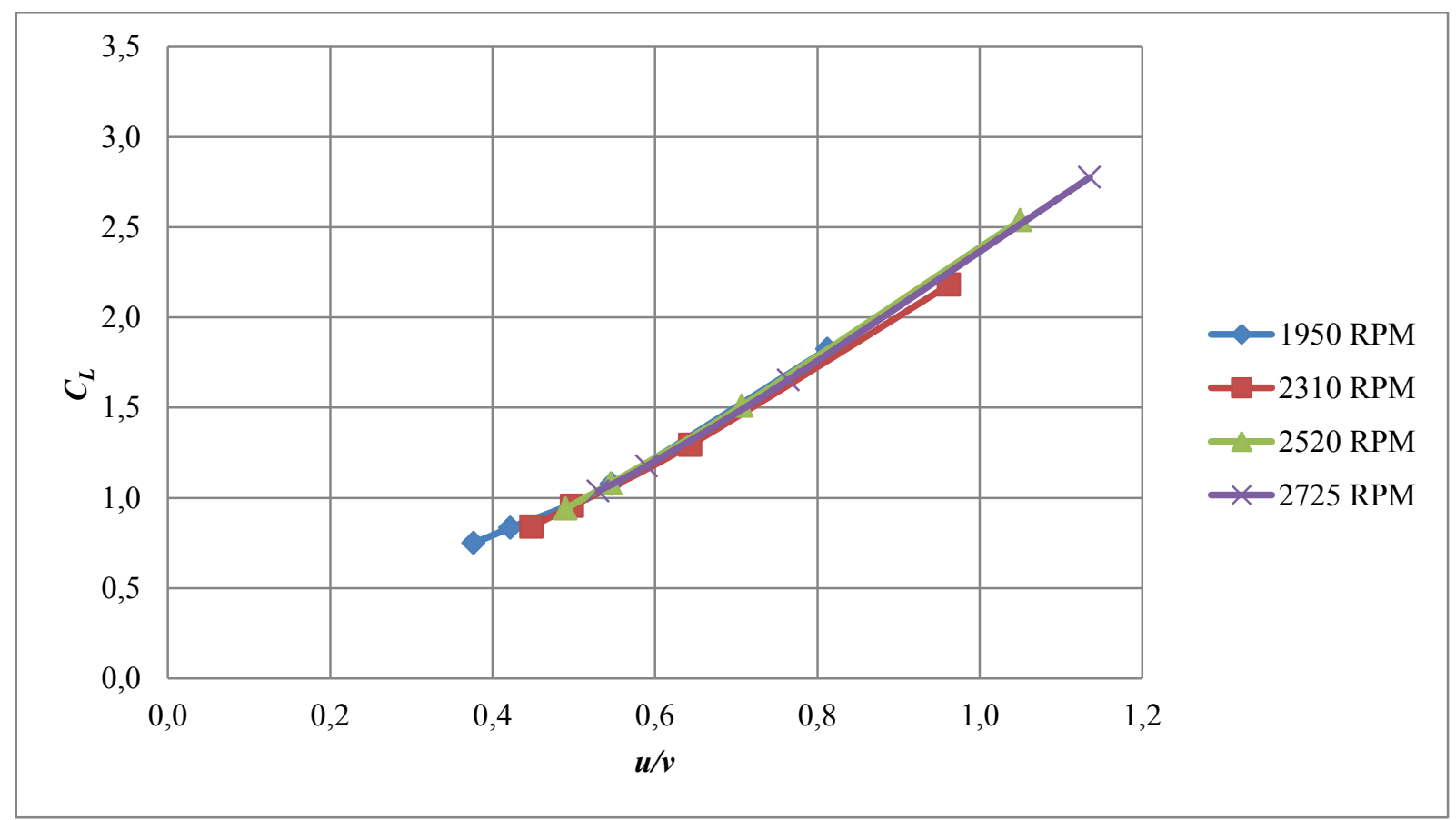

Figura A1.34 Variación del coeficiente de sustentación $C_{L}$ en función de la relación de velocidades $u / v$ para cada velocidad de giro $\omega$. 


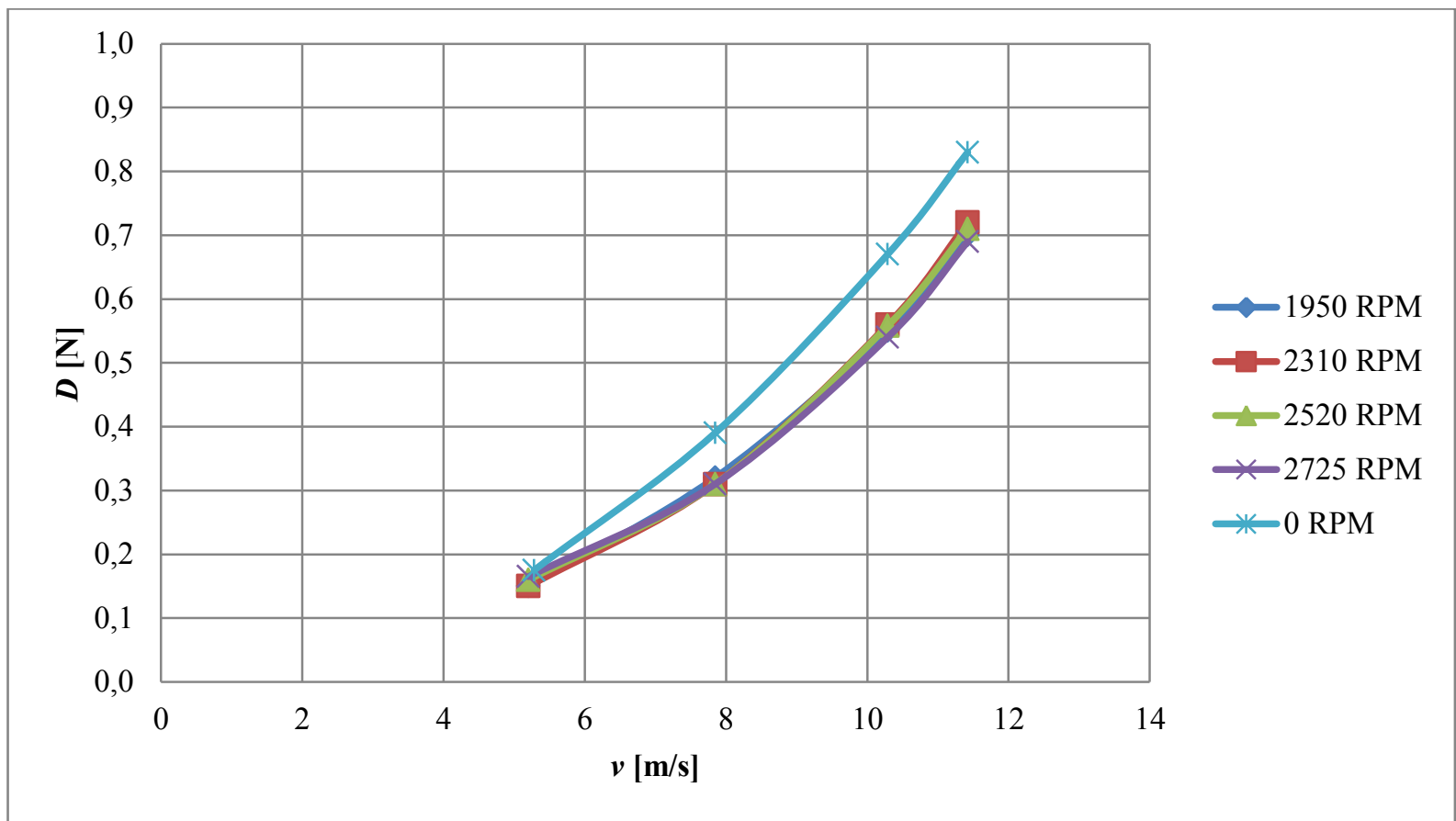

Figura A1.35 Variación de la resistencia $D$ en función de la velocidad de la corriente $v$ para cada velocidad de giro $\omega$.

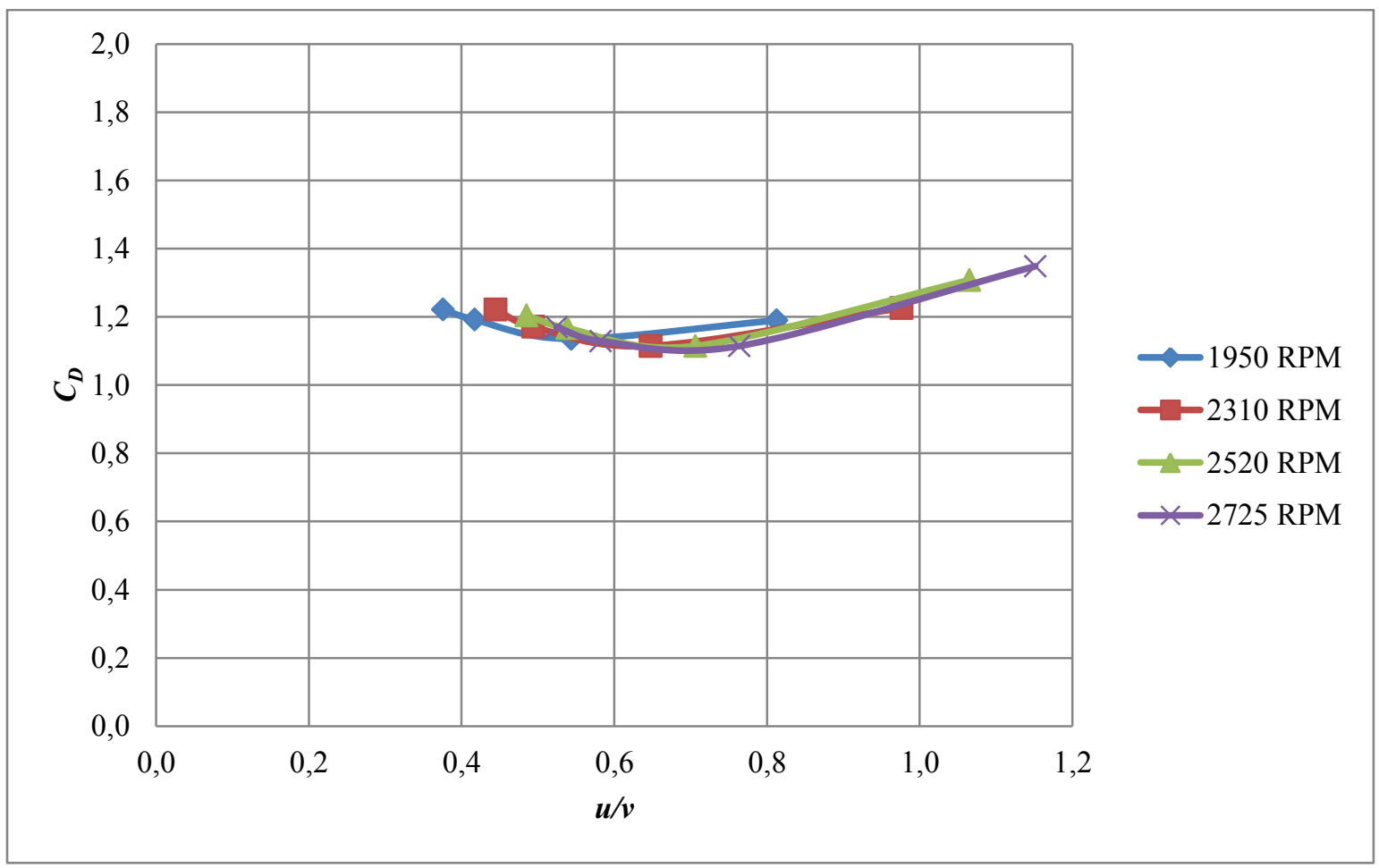

Figura A1.36 Variación del coeficiente de resistencia $C_{D}$ en función de la relación de velocidades $u / v$ para cada velocidad de giro $\omega$. 


\subsubsection{Cuadrado con aristas redondeadas}

Los resultados correspondientes a los ensayos de sustentación y resistencia se presentan en la tabla A1.11.

Tabla A1.11 Resultados correspondiente a los ensayos de sustentación y resistencia.

\begin{tabular}{|c|c|c|c|c|c|c|c|c|}
\hline \multicolumn{9}{|c|}{ Cuadrado con aristas redondeadas $(\phi=0,0415 \mathrm{~m})$} \\
\hline $\begin{array}{c}\omega \\
{[\mathrm{rpm}]}\end{array}$ & $\begin{array}{c}v \\
{[\mathrm{~m} / \mathrm{s}]}\end{array}$ & $\begin{array}{c}L \\
{[\mathrm{~N}]}\end{array}$ & $C_{L}$ & $u / v$ & $\begin{array}{c}v \\
{[\mathrm{~m} / \mathrm{s}]}\end{array}$ & $\begin{array}{c}D \\
{[\mathrm{~N}]}\end{array}$ & $C_{D}$ & $u / v$ \\
\hline \multirow[t]{4}{*}{0} & - & - & - & - & 5,17 & 0,12 & 1,02 & 0,00 \\
\hline & - & - & - & - & 7,86 & 0,28 & 1,03 & 0,00 \\
\hline & - & - & - & - & 10,53 & 0,49 & 1,01 & 0,00 \\
\hline & - & - & - & - & 11,80 & 0,62 & 1,01 & 0,00 \\
\hline \multirow[t]{4}{*}{1950} & 5,21 & 0,24 & 2,02 & 0,81 & 5,21 & 0,13 & 1,09 & 0,81 \\
\hline & 7,91 & 0,36 & 1,29 & 0,54 & 7,91 & 0,28 & 1,02 & 0,54 \\
\hline & 10,53 & 0,48 & 0,98 & 0,40 & 10,53 & 0,50 & 1,03 & 0,40 \\
\hline & 11,80 & 0,56 & 0,91 & 0,36 & 11,84 & 0,65 & 1,06 & 0,36 \\
\hline \multirow[t]{4}{*}{2310} & 5,17 & 0,28 & 2,35 & 0,97 & 5,21 & 0,15 & 1,22 & 0,96 \\
\hline & 7,88 & 0,43 & 1,56 & 0,64 & 7,91 & 0,28 & 1,02 & 0,63 \\
\hline & 10,51 & 0,56 & 1,15 & 0,48 & 10,53 & 0,52 & 1,06 & 0,48 \\
\hline & 11,78 & 0,64 & 1,04 & 0,43 & 11,80 & 0,67 & 1,09 & 0,43 \\
\hline \multirow[t]{4}{*}{2520} & 5,17 & 0,31 & 2,64 & 1,06 & 5,21 & 0,15 & 1,26 & 1,05 \\
\hline & 7,88 & 0,49 & 1,78 & 0,69 & 7,91 & 0,29 & 1,06 & 0,69 \\
\hline & 10,55 & 0,63 & 1,29 & 0,52 & 10,53 & 0,51 & 1,05 & 0,52 \\
\hline & 11,80 & 0,70 & 1,14 & 0,46 & 11,80 & 0,67 & 1,09 & 0,46 \\
\hline \multirow[t]{4}{*}{2725} & 5,17 & 0,33 & 2,81 & 1,15 & 5,21 & 0,16 & 1,34 & 1,14 \\
\hline & 7,88 & 0,52 & 1,90 & 0,75 & 7,86 & 0,30 & 1,11 & 0,75 \\
\hline & 10,51 & 0,68 & 1,40 & 0,56 & 10,51 & 0,52 & 1,06 & 0,56 \\
\hline & 11,84 & 0,75 & 1,21 & 0,50 & 11,84 & 0,64 & 1,04 & 0,50 \\
\hline
\end{tabular}




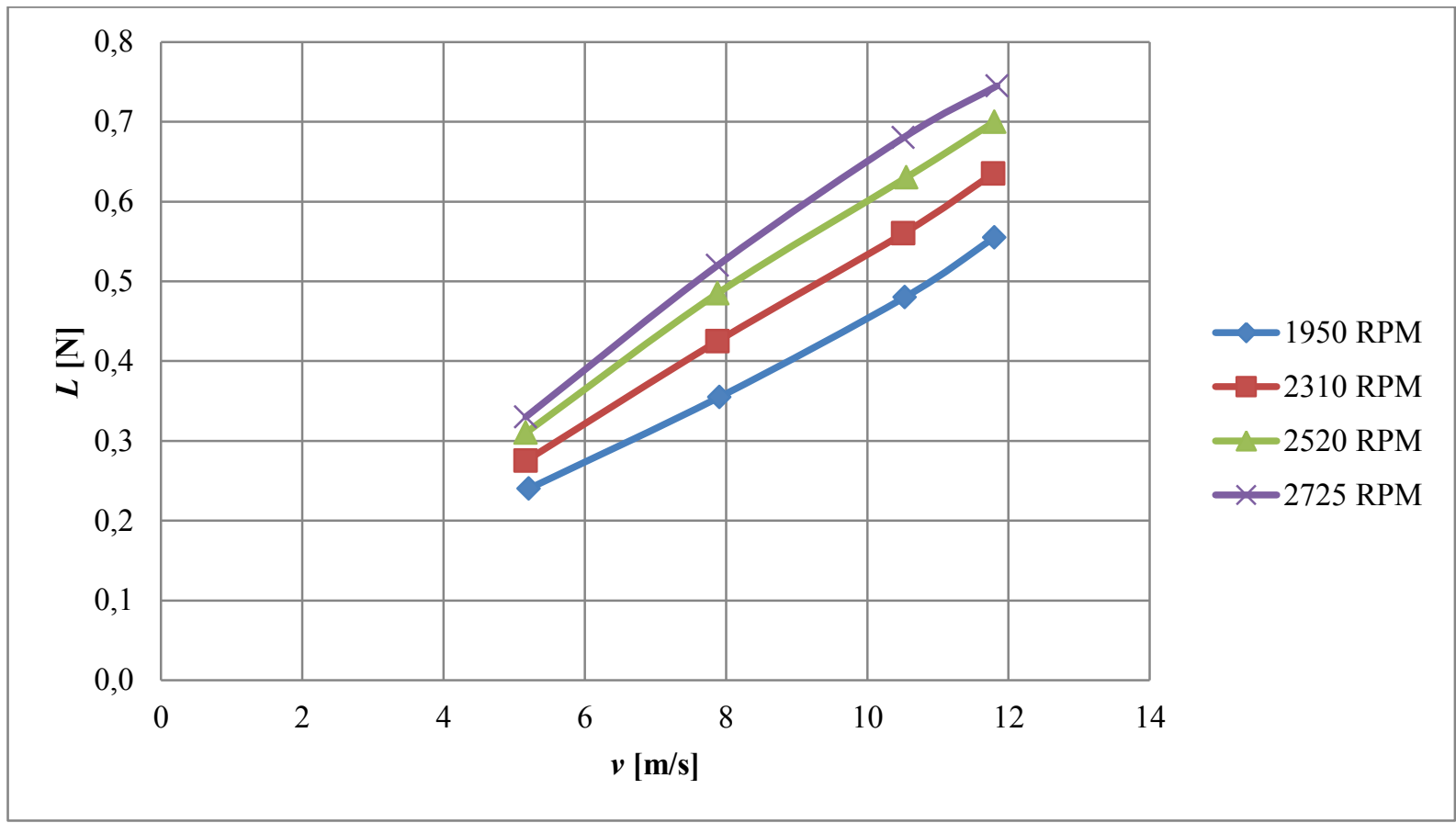

Figura A1.37 Variación de la sustentación $L$ en función de la velocidad de la corriente $v$ para cada velocidad de giro $\omega$.

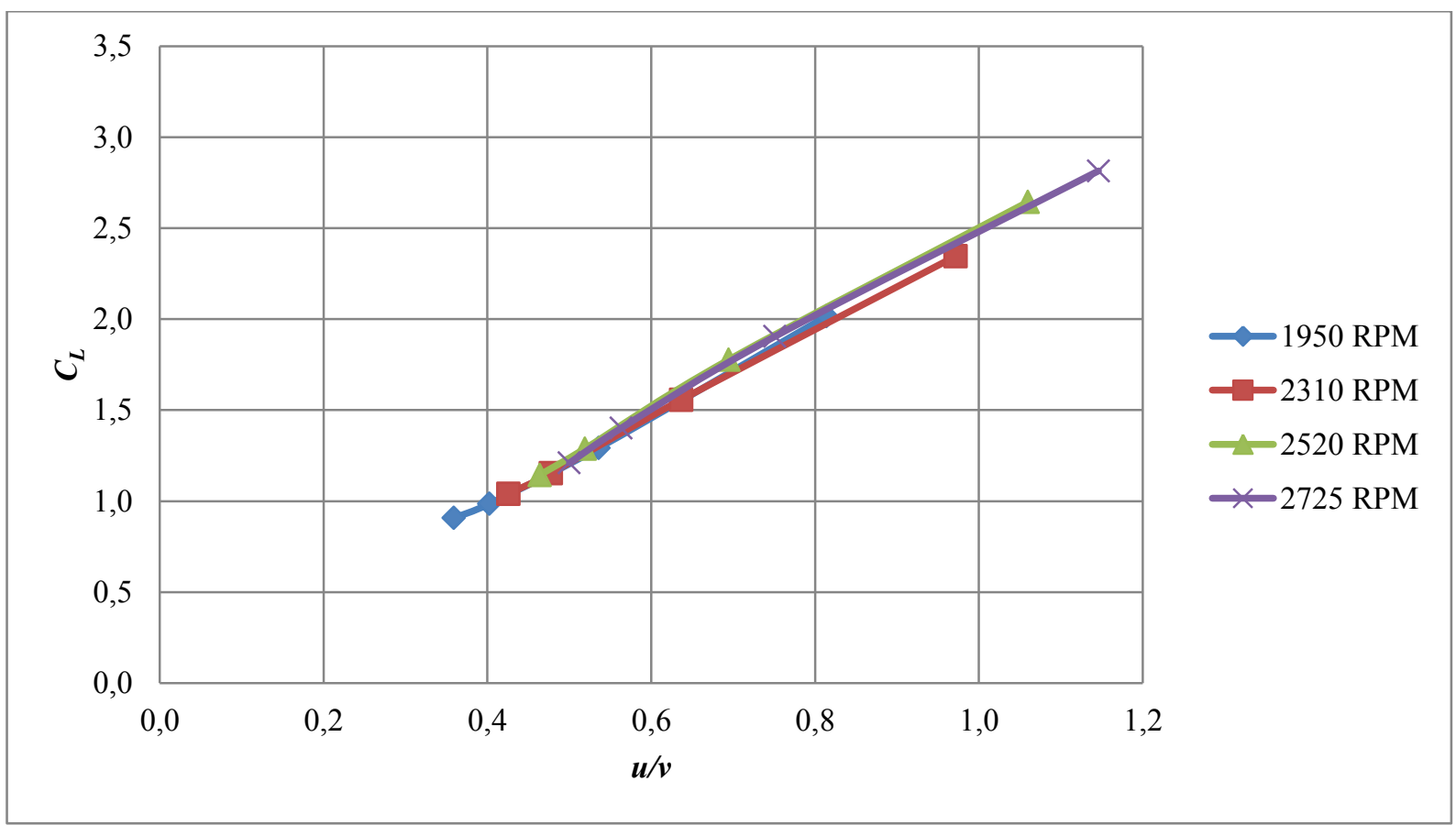

Figura A1.38 Variación del coeficiente de sustentación $C_{L}$ en función de la relación de velocidades $u / v$ para cada velocidad de giro $\omega$. 


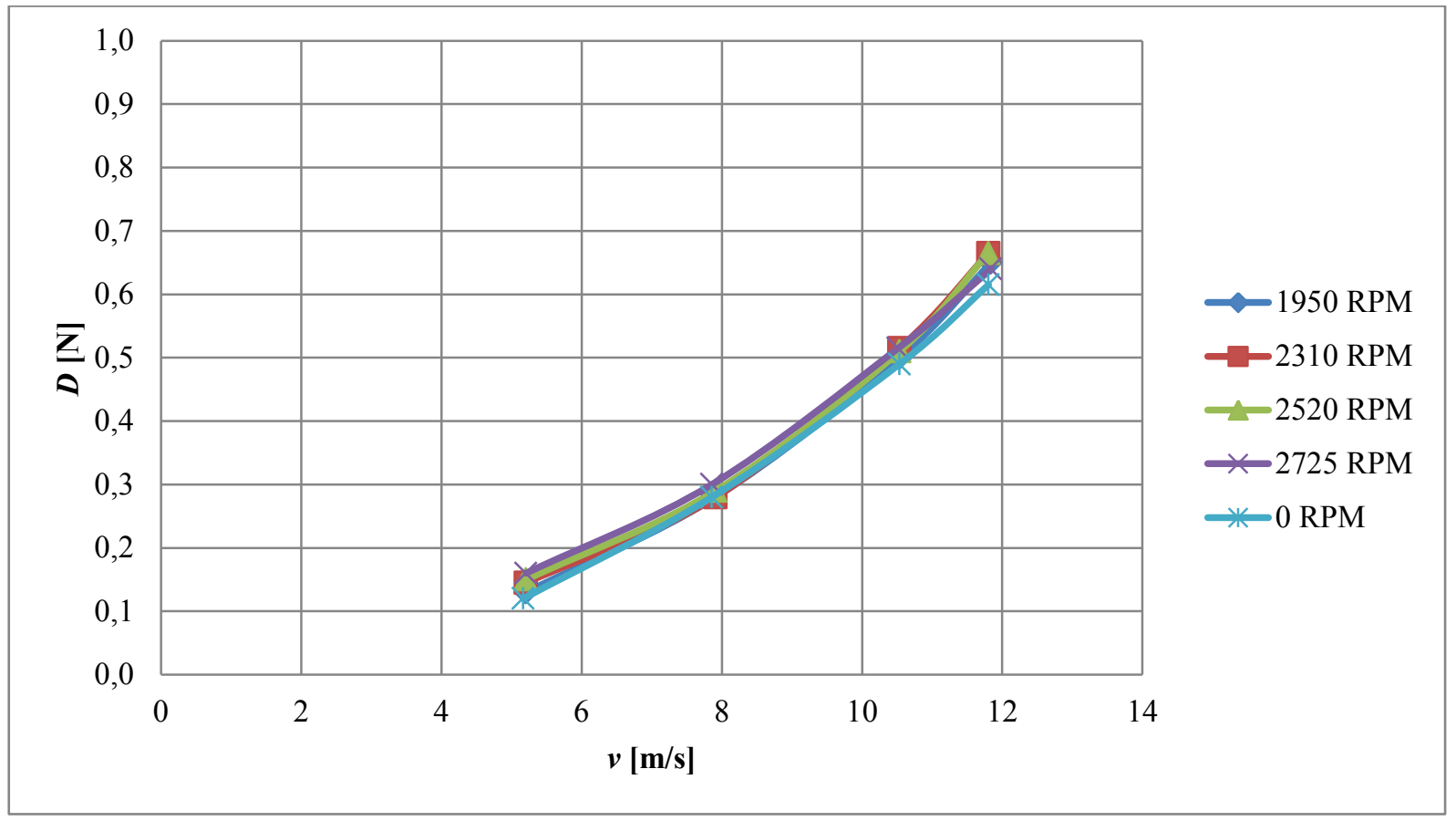

Figura A1.39 Variación de la resistencia $D$ en función de la velocidad de la corriente $v$ para cada velocidad de giro $\omega$.

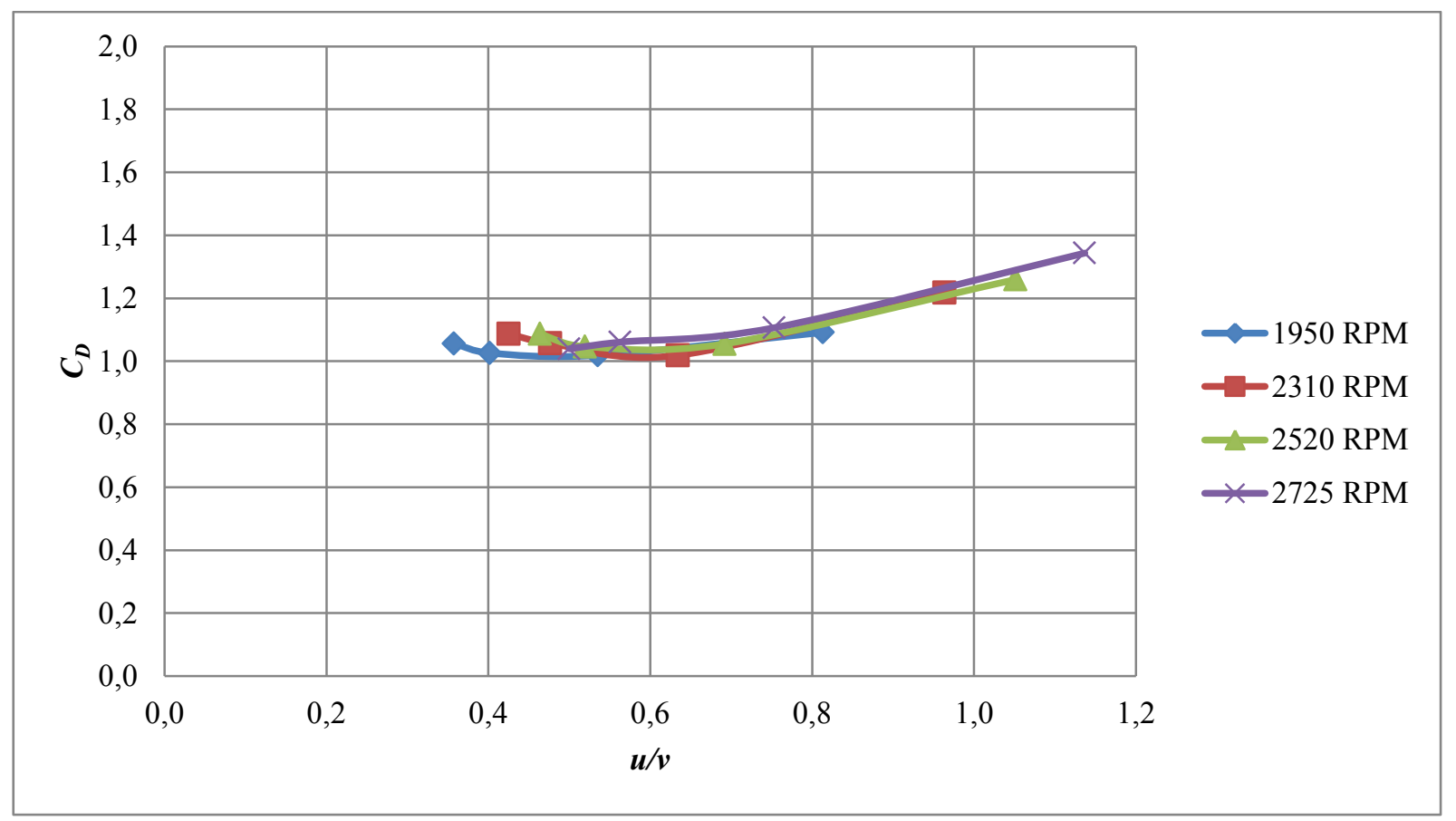

Figura A1.40 Variación del coeficiente de resistencia $C_{\boldsymbol{D}}$ en función de la relación de velocidades $u / v$ para cada velocidad de giro $\omega$. 


\subsubsection{Triángulo}

Los resultados correspondientes a los ensayos de sustentación y resistencia se presentan en la tabla A1.12.

Tabla A1.12 Resultados correspondiente a los ensayos de sustentación y resistencia.

\begin{tabular}{|c|c|c|c|c|c|c|c|c|}
\hline \multicolumn{9}{|c|}{ Triángulo $(\phi=0,0325 \mathrm{~m})$} \\
\hline $\begin{array}{c}\omega \\
{[\mathrm{rpm}]}\end{array}$ & $\begin{array}{c}v \\
{[\mathbf{m} / \mathbf{s}]}\end{array}$ & $\begin{array}{c}L \\
{[\mathbf{N}]}\end{array}$ & $C_{L}$ & $u / v$ & $\begin{array}{c}v \\
{[\mathrm{~m} / \mathrm{s}]}\end{array}$ & $\begin{array}{c}D \\
{[\mathbf{N}]}\end{array}$ & $C_{D}$ & $u / v$ \\
\hline \multirow[t]{4}{*}{0} & - & - & - & - & 5,15 & 0,18 & 1,91 & 0,00 \\
\hline & - & - & - & - & 7,84 & 0,42 & 1,95 & 0,00 \\
\hline & - & - & - & - & 10,34 & 0,74 & 2,00 & 0,00 \\
\hline & - & - & - & - & 11,59 & 0,94 & 2,01 & 0,00 \\
\hline \multirow[t]{4}{*}{1950} & 5,20 & 0,14 & 1,50 & 0,64 & 5,23 & 0,13 & 1,37 & 0,63 \\
\hline & 7,89 & 0,25 & 1,14 & 0,42 & 7,73 & 0,31 & 1,50 & 0,43 \\
\hline & 10,51 & 0,37 & 0,96 & 0,32 & 10,34 & 0,66 & 1,77 & 0,32 \\
\hline & 11,67 & 0,44 & 0,94 & 0,28 & 11,70 & 0,82 & 1,73 & 0,28 \\
\hline \multirow[t]{4}{*}{2310} & 5,20 & 0,17 & 1,82 & 0,76 & 5,23 & 0,13 & 1,32 & 0,75 \\
\hline & 7,89 & 0,27 & 1,25 & 0,50 & 7,86 & 0,31 & 1,45 & 0,50 \\
\hline & 10,41 & 0,41 & 1,09 & 0,38 & 10,42 & 0,66 & 1,76 & 0,38 \\
\hline & 11,71 & 0,47 & 0,99 & 0,34 & 11,77 & 0,85 & 1,77 & 0,33 \\
\hline \multirow[t]{4}{*}{2520} & 5,20 & 0,18 & 1,93 & 0,83 & 5,23 & 0,13 & 1,37 & 0,82 \\
\hline & 7,89 & 0,29 & 1,32 & 0,54 & 7,94 & 0,31 & 1,42 & 0,54 \\
\hline & 10,49 & 0,44 & 1,14 & 0,41 & 10,50 & 0,65 & 1,69 & 0,41 \\
\hline & 11,67 & 0,50 & 1,06 & 0,37 & 11,74 & 0,85 & 1,77 & 0,37 \\
\hline \multirow[t]{4}{*}{2725} & 5,20 & 0,20 & 2,14 & 0,89 & 5,23 & 0,13 & 1,32 & 0,89 \\
\hline & 7,89 & 0,31 & 1,42 & 0,59 & 7,94 & 0,31 & 1,40 & 0,58 \\
\hline & 10,49 & 0,45 & 1,17 & 0,44 & 10,54 & 0,63 & 1,64 & 0,44 \\
\hline & 11,71 & 0,54 & 1,13 & 0,40 & 11,77 & 0,82 & 1,70 & 0,39 \\
\hline
\end{tabular}




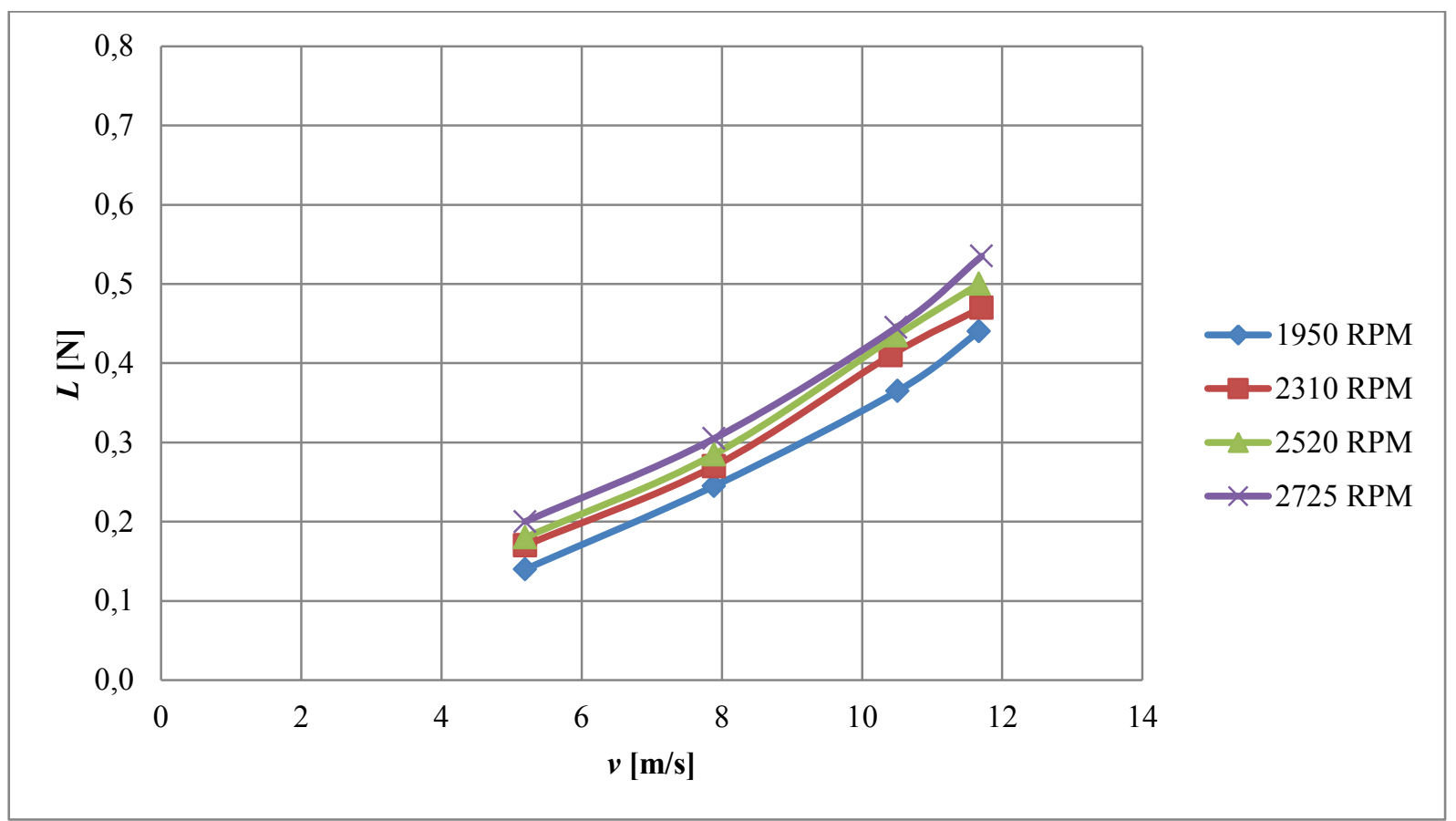

Figura A1.41 Variación de la sustentación $L$ en función de la velocidad de la corriente $v$ para cada velocidad de giro $\omega$.

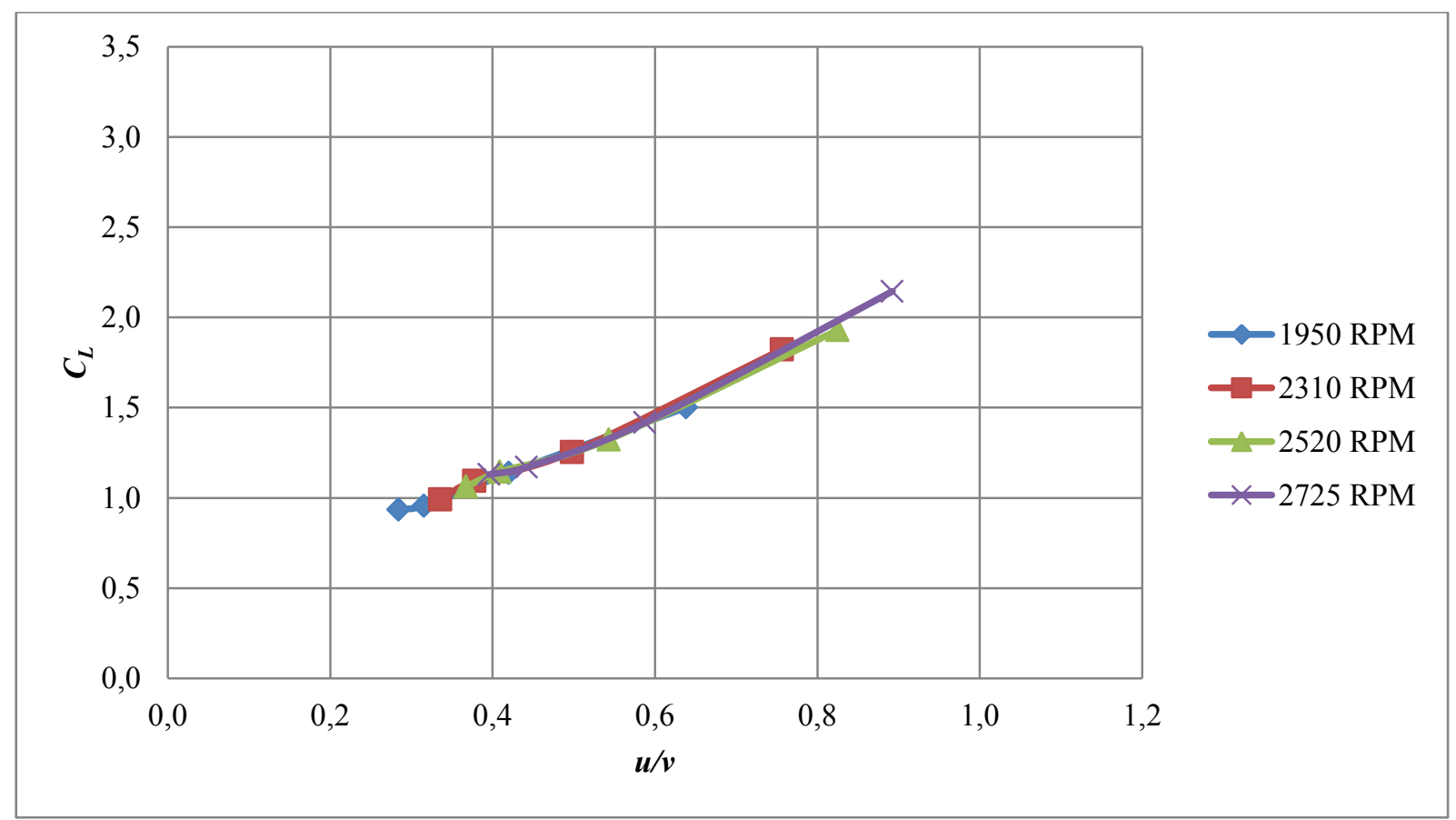

Figura A1.42 Variación del coeficiente de sustentación $C_{L}$ en función de la relación de velocidades $u / v$ para cada velocidad de giro $\omega$. 


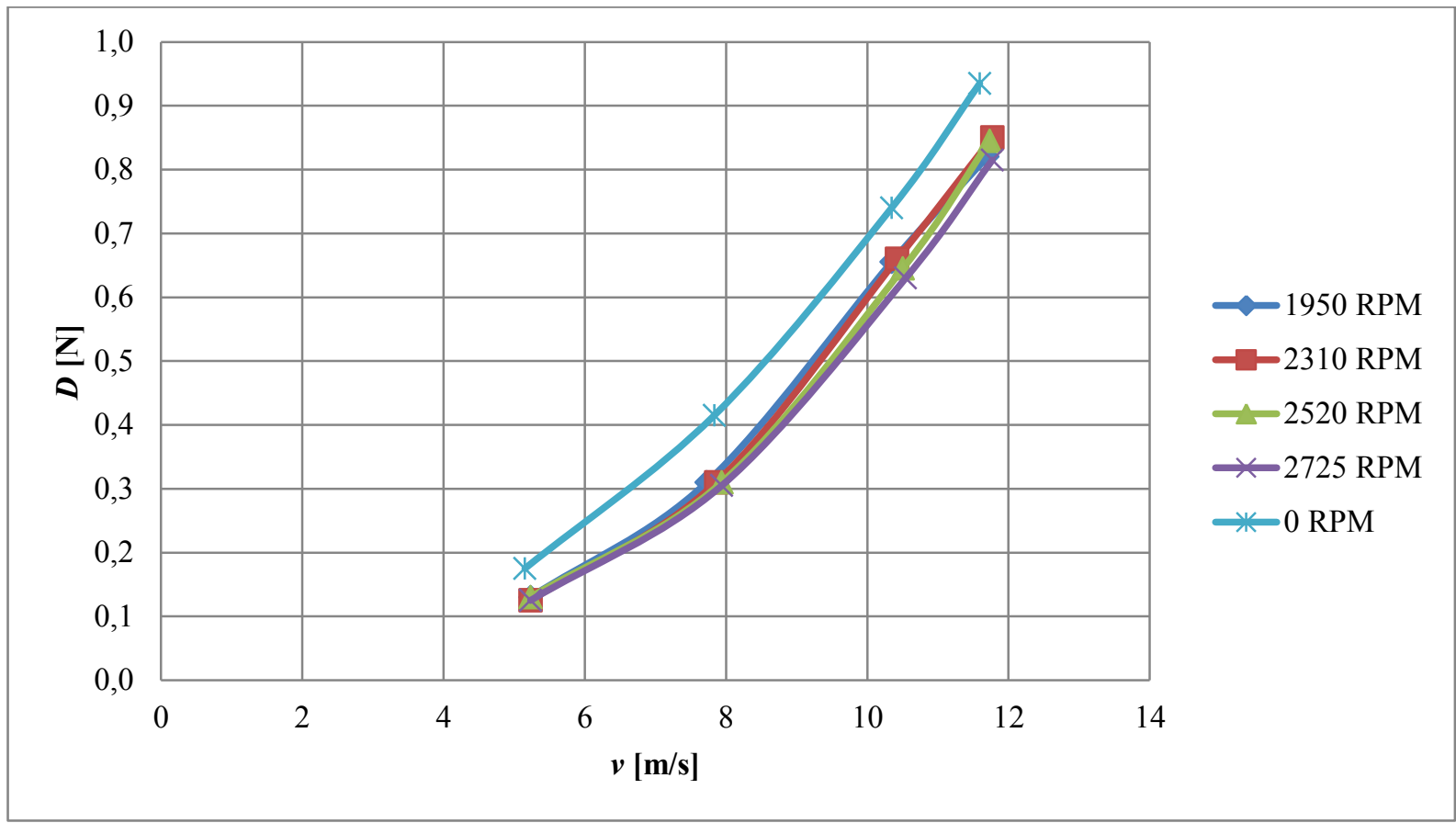

Figura A1.43 Variación de la resistencia $D$ en función de la velocidad de la corriente $v$ para cada velocidad de giro $\omega$.

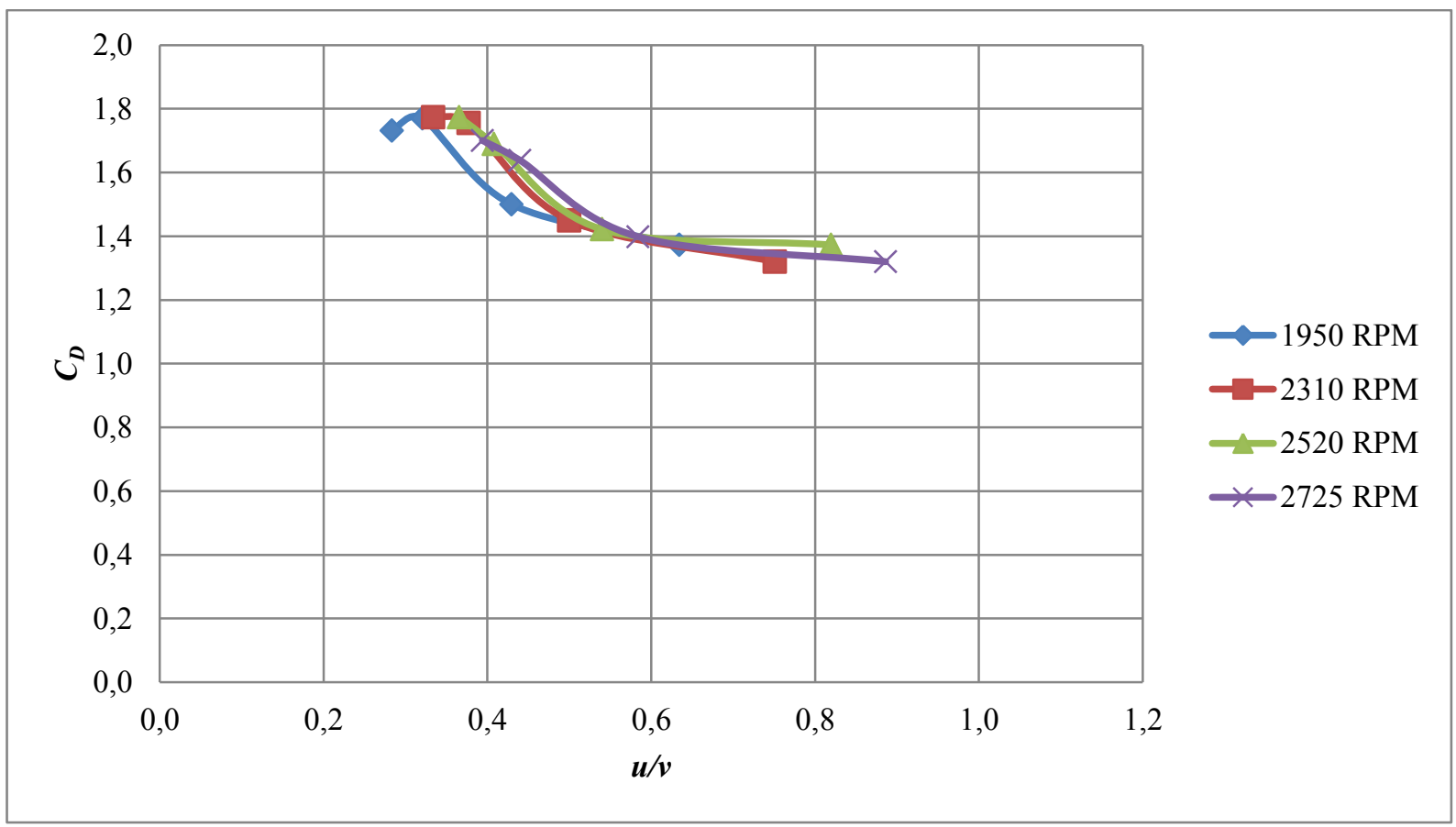

Figura A1.44 Variación del coeficiente de resistencia $C_{\boldsymbol{D}}$ en función de la relación de velocidades $u / v$ para cada velocidad de giro $\omega$. 


\subsubsection{Triángulo con aristas redondeadas}

Los resultados correspondientes a los ensayos de sustentación y resistencia se presentan en la tabla A1.13.

Tabla A1.13 Resultados correspondiente a los ensayos de sustentación y resistencia.

\begin{tabular}{|c|c|c|c|c|c|c|c|c|}
\hline \multicolumn{9}{|c|}{ Triángulo con aristas redondeadas $(\phi=0,0295 \mathrm{~m})$} \\
\hline $\begin{array}{c}\omega \\
{[\mathrm{rpm}]}\end{array}$ & $\begin{array}{c}v \\
{[\mathrm{~m} / \mathrm{s}]}\end{array}$ & $\begin{array}{c}\boldsymbol{L} \\
{[\mathrm{N}]}\end{array}$ & $C_{L}$ & $u / v$ & $\begin{array}{c}v \\
{[\mathrm{~m} / \mathrm{s}]}\end{array}$ & $\begin{array}{c}D \\
{[\mathrm{~N}]}\end{array}$ & $C_{D}$ & $u / v$ \\
\hline \multirow[t]{4}{*}{0} & - & - & - & - & 5,20 & 0,13 & 1,48 & 0,00 \\
\hline & - & - & - & - & 7,85 & 0,29 & 1,48 & 0,00 \\
\hline & - & - & - & - & 10,60 & 0,52 & 1,46 & 0,00 \\
\hline & - & - & - & - & 11,82 & 0,65 & 1,49 & 0,00 \\
\hline \multirow[t]{4}{*}{1950} & 5,21 & 0,14 & 1,59 & 0,58 & 5,20 & 0,10 & 1,12 & 0,58 \\
\hline & 7,91 & 0,23 & 1,15 & 0,38 & 7,85 & 0,25 & 1,30 & 0,38 \\
\hline & 10,57 & 0,32 & 0,92 & 0,28 & 10,60 & 0,46 & 1,31 & 0,28 \\
\hline & 11,84 & 0,38 & 0,86 & 0,25 & 11,79 & 0,57 & 1,31 & 0,26 \\
\hline \multirow[t]{4}{*}{2310} & 5,21 & 0,16 & 1,89 & 0,69 & 5,20 & 0,09 & 1,06 & 0,69 \\
\hline & 7,94 & 0,25 & 1,27 & 0,45 & 7,95 & 0,24 & 1,21 & 0,45 \\
\hline & 10,55 & 0,37 & 1,05 & 0,34 & 10,56 & 0,46 & 1,32 & 0,34 \\
\hline & 11,84 & 0,43 & 0,97 & 0,30 & 11,82 & 0,56 & 1,28 & 0,30 \\
\hline \multirow[t]{4}{*}{2520} & 5,21 & 0,18 & 2,13 & 0,75 & 5,20 & 0,09 & 1,06 & 0,75 \\
\hline & 7,94 & 0,27 & 1,37 & 0,49 & 7,95 & 0,23 & 1,14 & 0,49 \\
\hline & 10,55 & 0,40 & 1,14 & 0,37 & 10,56 & 0,47 & 1,33 & 0,37 \\
\hline & 11,84 & 0,45 & 1,03 & 0,33 & 11,86 & 0,59 & 1,33 & 0,33 \\
\hline \multirow[t]{4}{*}{2725} & 5,17 & 0,19 & 2,28 & 0,81 & 5,24 & 0,09 & 1,05 & 0,80 \\
\hline & 7,91 & 0,29 & 1,46 & 0,53 & 7,95 & 0,22 & 1,09 & 0,53 \\
\hline & 10,57 & 0,43 & 1,22 & 0,40 & 10,58 & 0,46 & 1,30 & 0,40 \\
\hline & 11,84 & 0,48 & 1,09 & 0,36 & 11,80 & 0,58 & 1,32 & 0,36 \\
\hline
\end{tabular}




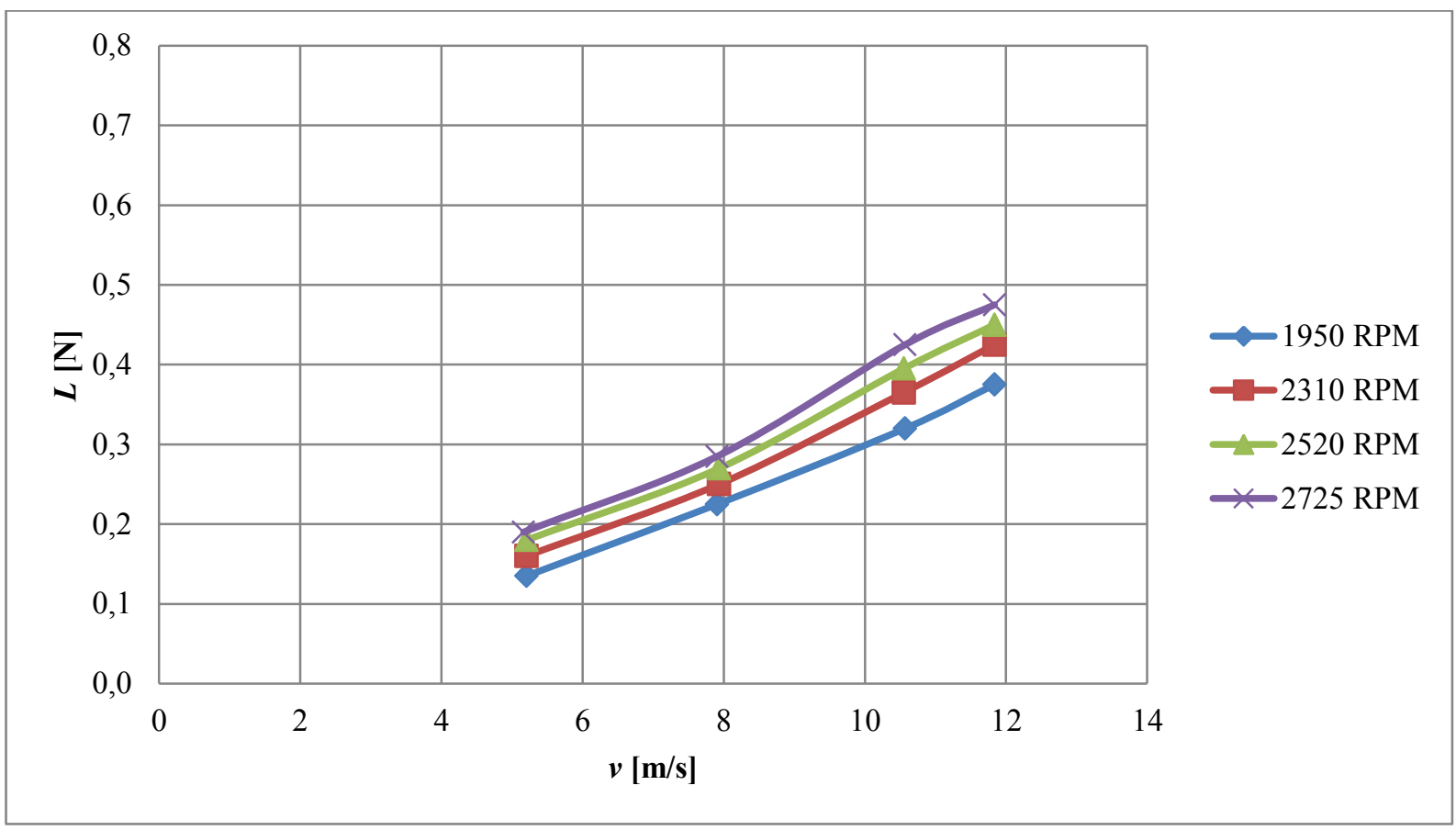

Figura A1.45 Variación de la sustentación $L$ en función de la velocidad de la corriente $v$ para cada velocidad de giro $\omega$.

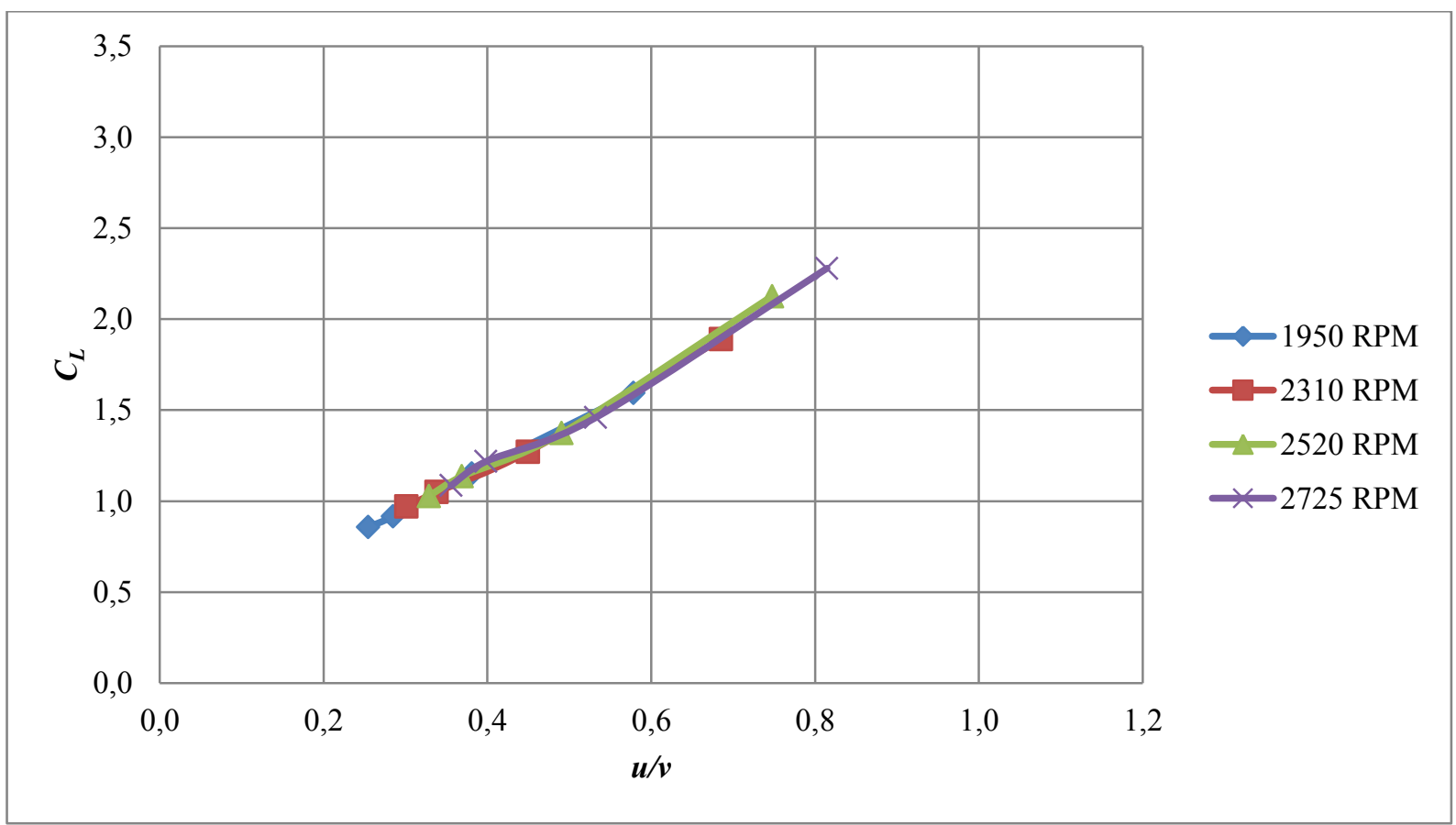

Figura A1.46 Variación del coeficiente de sustentación $C_{L}$ en función de la relación de velocidades $u / v$ para cada velocidad de giro $\omega$. 


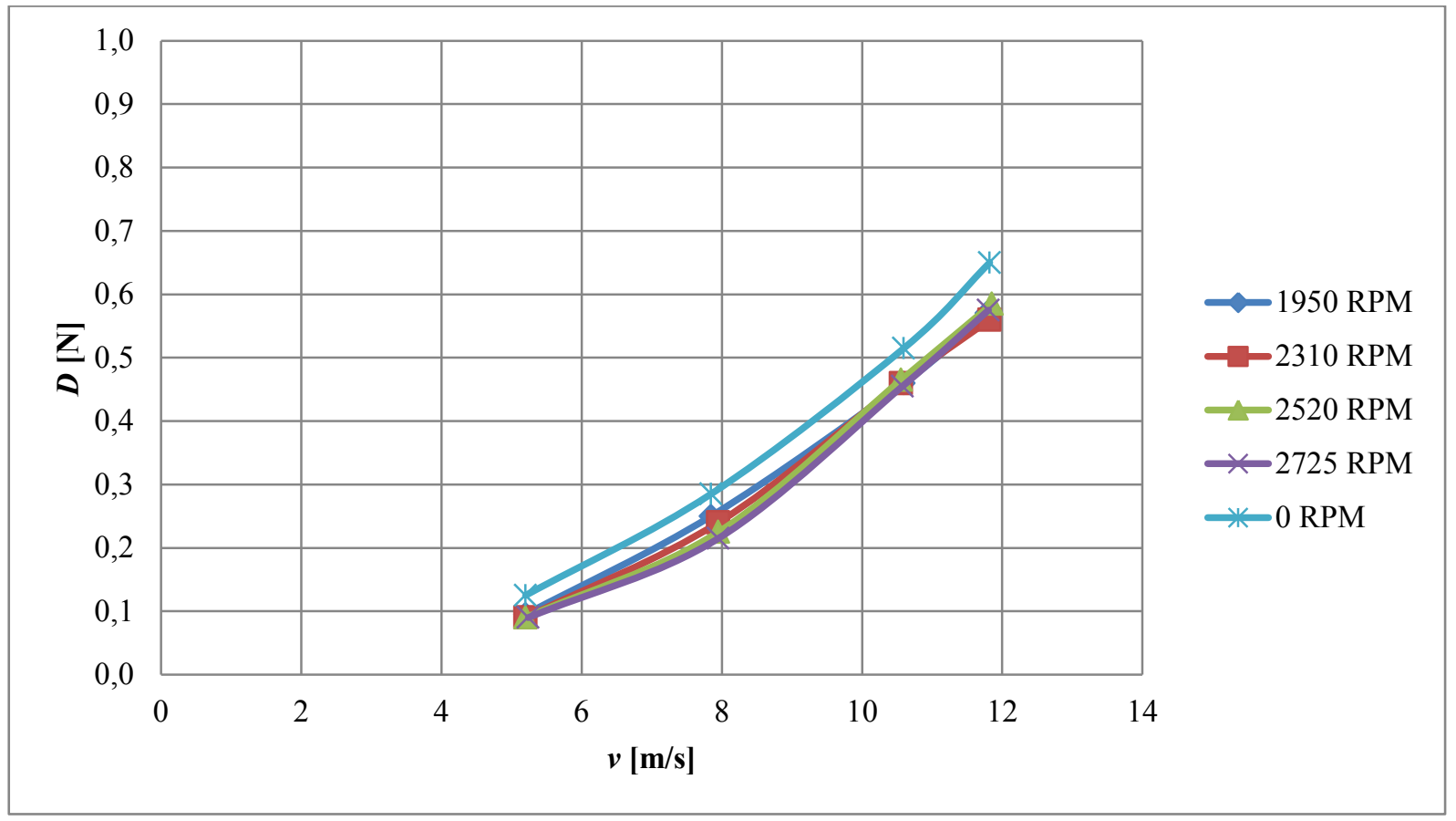

Figura A1.47 Variación de la resistencia $D$ en función de la velocidad de la corriente $v$ para cada velocidad de giro $\omega$.

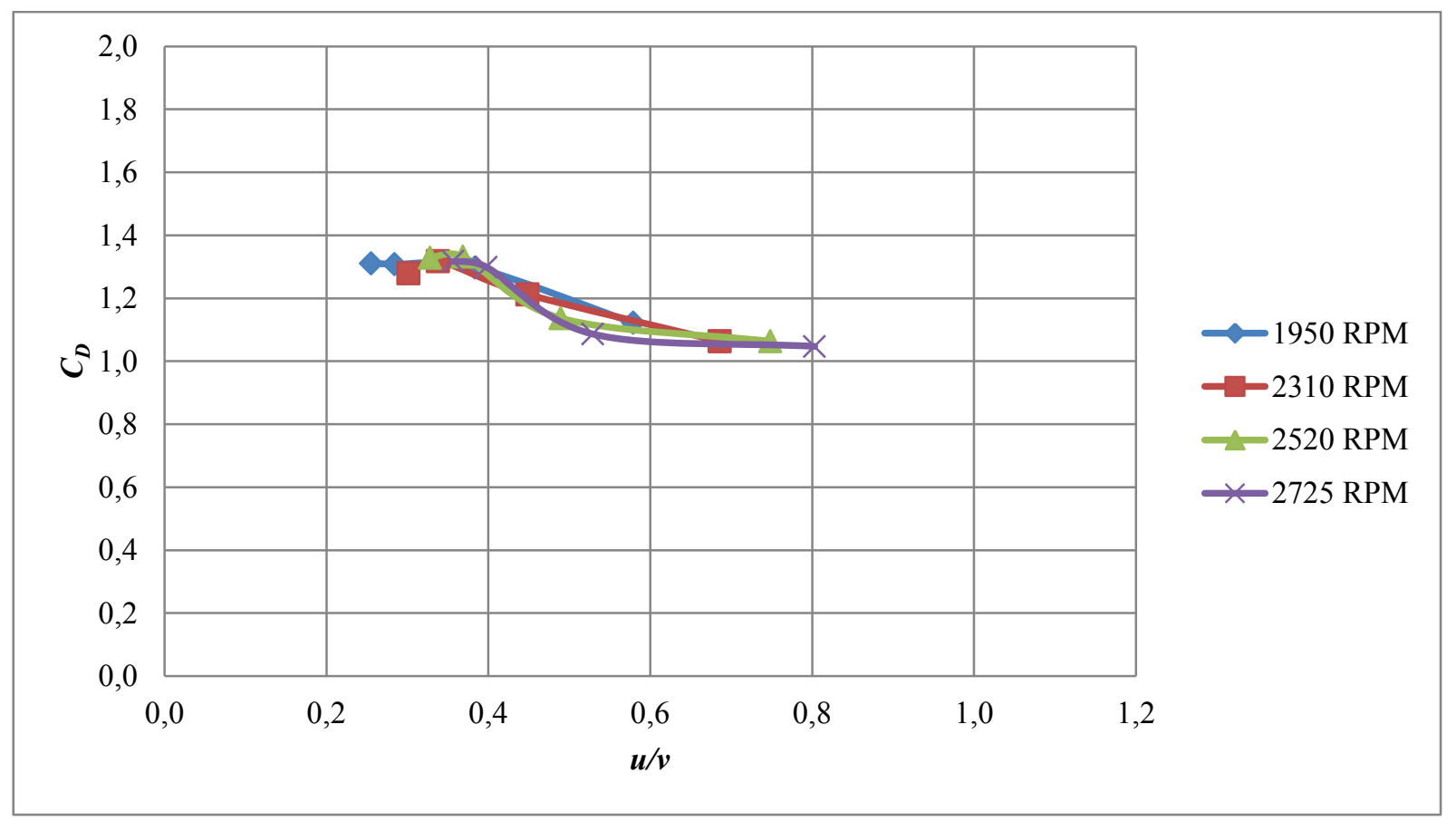

Figura A1.48 Variación del coeficiente de resistencia $C_{D}$ en función de la relación de velocidades $u / v$ para cada velocidad de giro $\omega$. 


\subsubsection{Placa plana gruesa}

Los resultados correspondientes a los ensayos de sustentación y resistencia se presentan en la tabla A1.14.

Tabla A1.14 Resultados correspondiente a los ensayos de sustentación y resistencia.

\begin{tabular}{|c|c|c|c|c|c|c|c|c|}
\hline \multicolumn{9}{|c|}{ Placa plana gruesa $(\phi=0,035 \mathrm{~m})$} \\
\hline $\begin{array}{c}\omega \\
{[\mathrm{rpm}]}\end{array}$ & $\begin{array}{c}v \\
{[\mathbf{m} / \mathbf{s}]}\end{array}$ & $\begin{array}{c}L \\
{[\mathbf{N}]}\end{array}$ & $C_{L}$ & $u / v$ & $\begin{array}{c}v \\
{[\mathbf{m} / \mathbf{s}]}\end{array}$ & $\begin{array}{c}D \\
{[\mathrm{~N}]}\end{array}$ & $C_{D}$ & $u / v$ \\
\hline \multirow[t]{4}{*}{0} & - & - & - & - & 5,23 & 0,18 & 1,77 & 0,00 \\
\hline & - & - & - & - & 7,89 & 0,42 & 1,81 & 0,00 \\
\hline & - & - & - & - & 10,47 & 0,75 & 1,84 & 0,00 \\
\hline & - & - & - & - & 11,70 & 0,94 & 1,83 & 0,00 \\
\hline \multirow[t]{4}{*}{1950} & 5,15 & 0,14 & 1,42 & 0,69 & 5,19 & 0,10 & 1,00 & 0,69 \\
\hline & 7,89 & 0,27 & 1,14 & 0,45 & 7,94 & 0,28 & 1,19 & 0,45 \\
\hline & 10,55 & 0,46 & 1,10 & 0,34 & 10,58 & 0,50 & 1,20 & 0,34 \\
\hline & 11,77 & 0,55 & 1,07 & 0,30 & 11,84 & 0,62 & 1,19 & 0,30 \\
\hline \multirow[t]{4}{*}{2310} & 5,15 & 0,16 & 1,57 & 0,82 & 5,35 & 0,11 & 0,98 & 0,79 \\
\hline & 7,89 & 0,29 & 1,25 & 0,54 & 7,91 & 0,29 & 1,24 & 0,53 \\
\hline & 10,47 & 0,47 & 1,15 & 0,40 & 10,58 & 0,51 & 1,22 & 0,40 \\
\hline & 11,81 & 0,59 & 1,13 & 0,36 & 11,84 & 0,63 & 1,21 & 0,36 \\
\hline \multirow[t]{4}{*}{2520} & 5,15 & 0,17 & 1,72 & 0,90 & 5,35 & 0,10 & 0,94 & 0,86 \\
\hline & 7,89 & 0,31 & 1,32 & 0,59 & 7,94 & 0,28 & 1,19 & 0,58 \\
\hline & 10,55 & 0,49 & 1,18 & 0,44 & 10,54 & 0,51 & 1,23 & 0,44 \\
\hline & 11,84 & 0,59 & 1,13 & 0,39 & 11,81 & 0,63 & 1,21 & 0,39 \\
\hline \multirow[t]{4}{*}{2725} & 5,15 & 0,18 & 1,82 & 0,97 & 5,35 & 0,09 & 0,84 & 0,93 \\
\hline & 7,89 & 0,33 & 1,42 & 0,63 & 7,89 & 0,27 & 1,14 & 0,63 \\
\hline & 10,47 & 0,50 & 1,23 & 0,48 & 10,54 & 0,54 & 1,29 & 0,47 \\
\hline & 11,81 & 0,60 & 1,16 & 0,42 & 11,77 & 0,66 & 1,28 & 0,42 \\
\hline
\end{tabular}




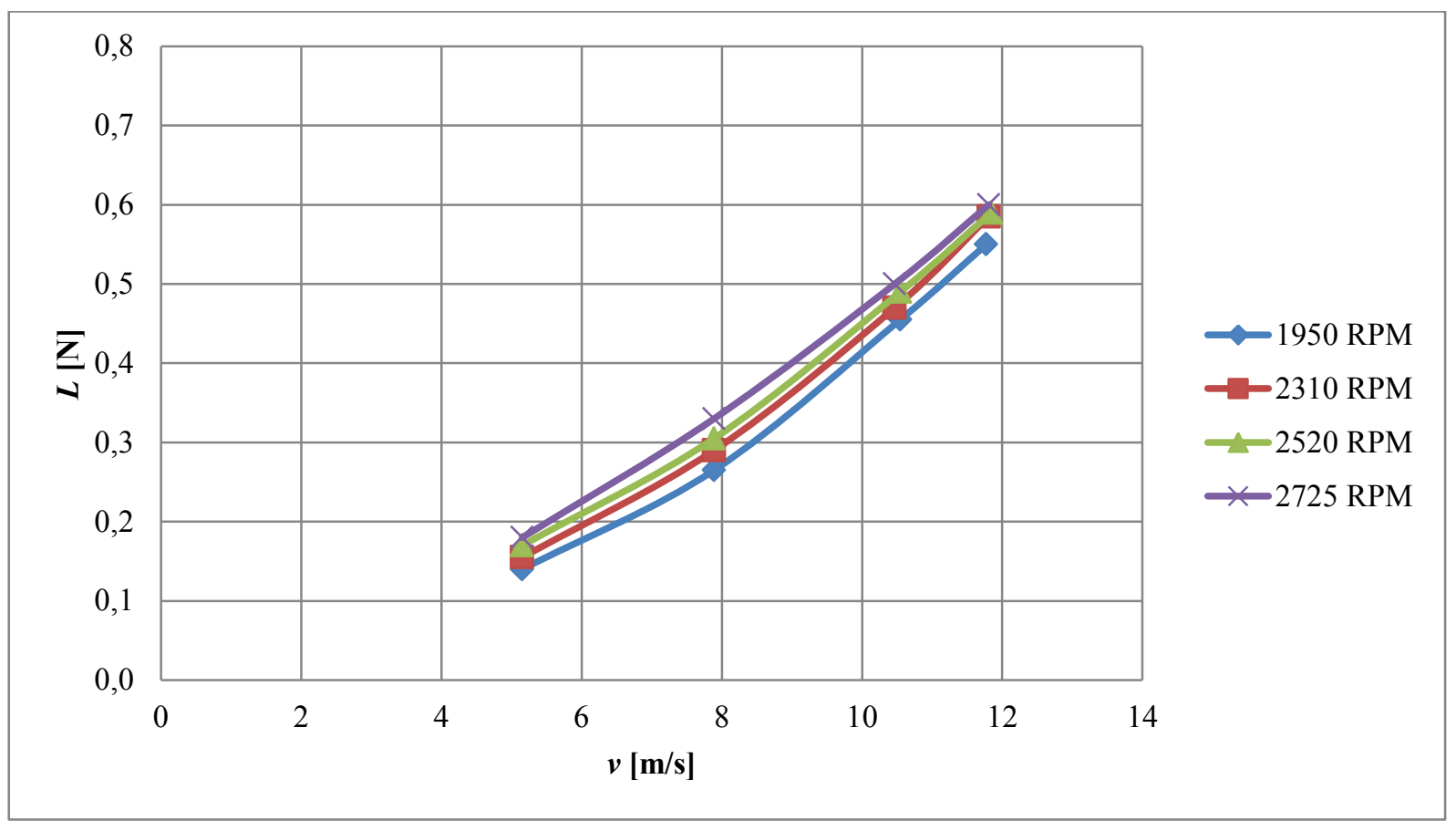

Figura A1.49 Variación de la sustentación $L$ en función de la velocidad de la corriente $v$ para cada velocidad de giro $\omega$.

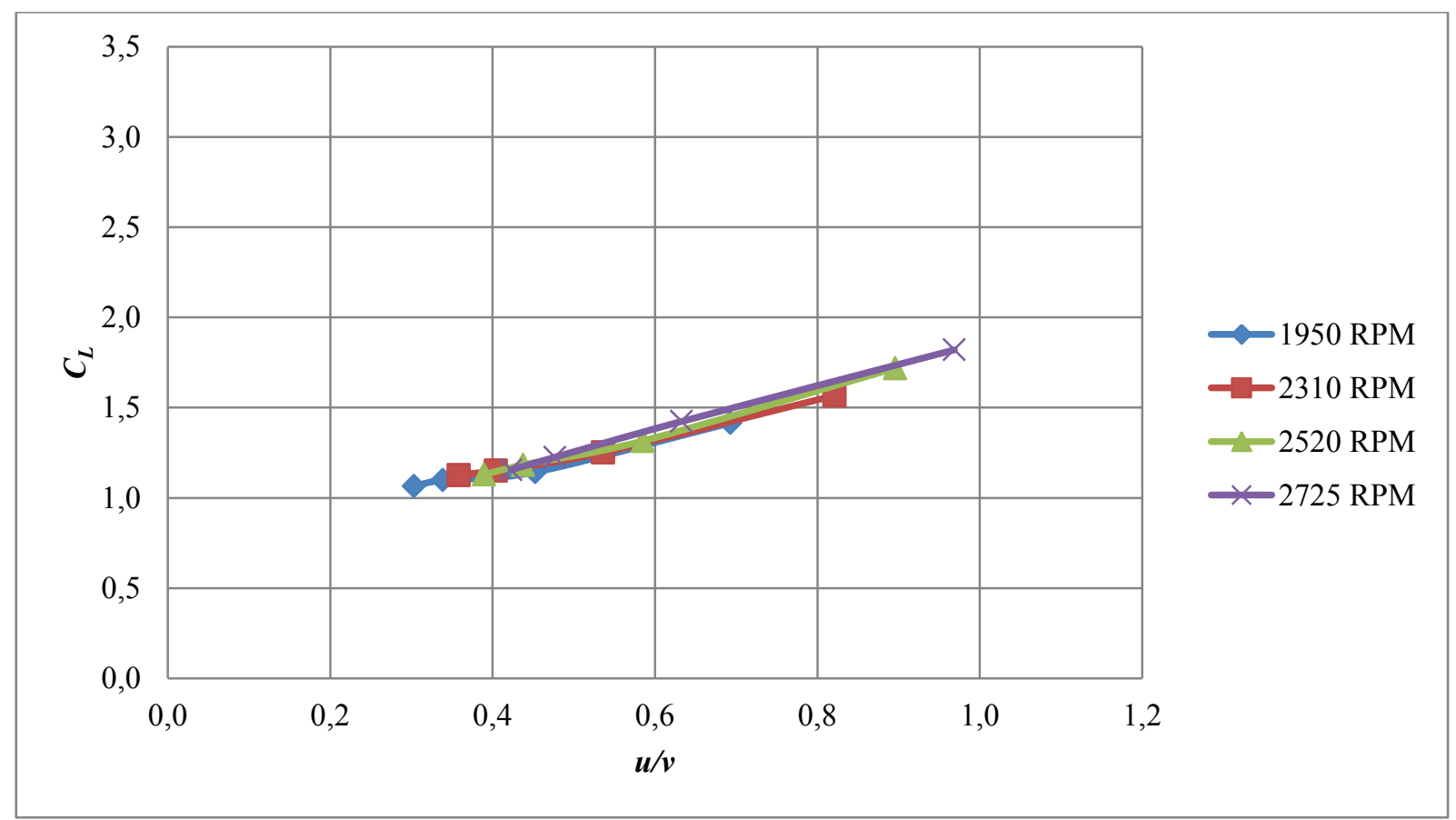

Figura A1.50 Variación del coeficiente de sustentación $C_{L}$ en función de la relación de velocidades $u / v$ para cada velocidad de giro $\omega$. 


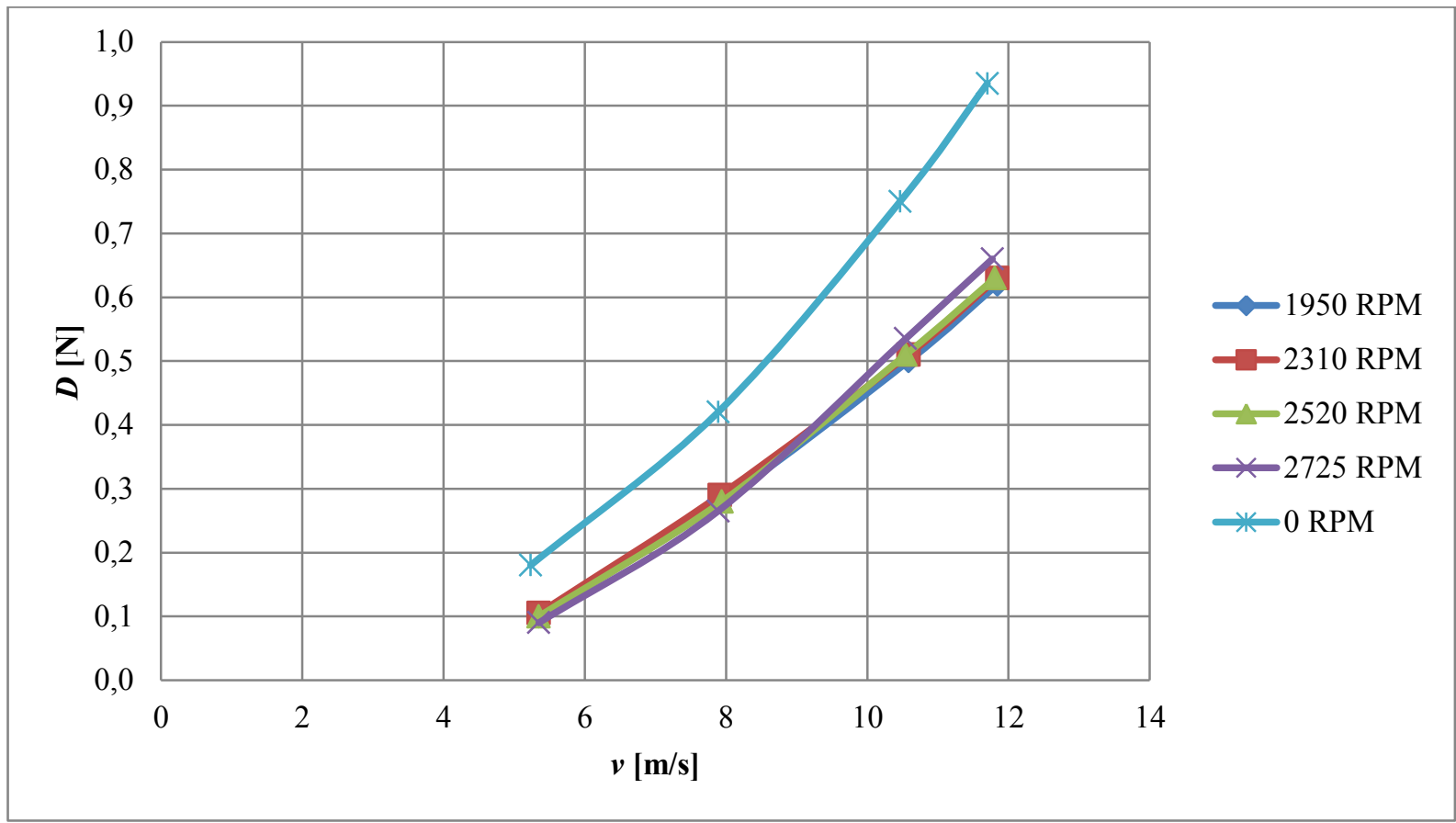

Figura A1.51 Variación de la resistencia $D$ en función de la velocidad de la corriente $v$ para cada velocidad de giro $\omega$.

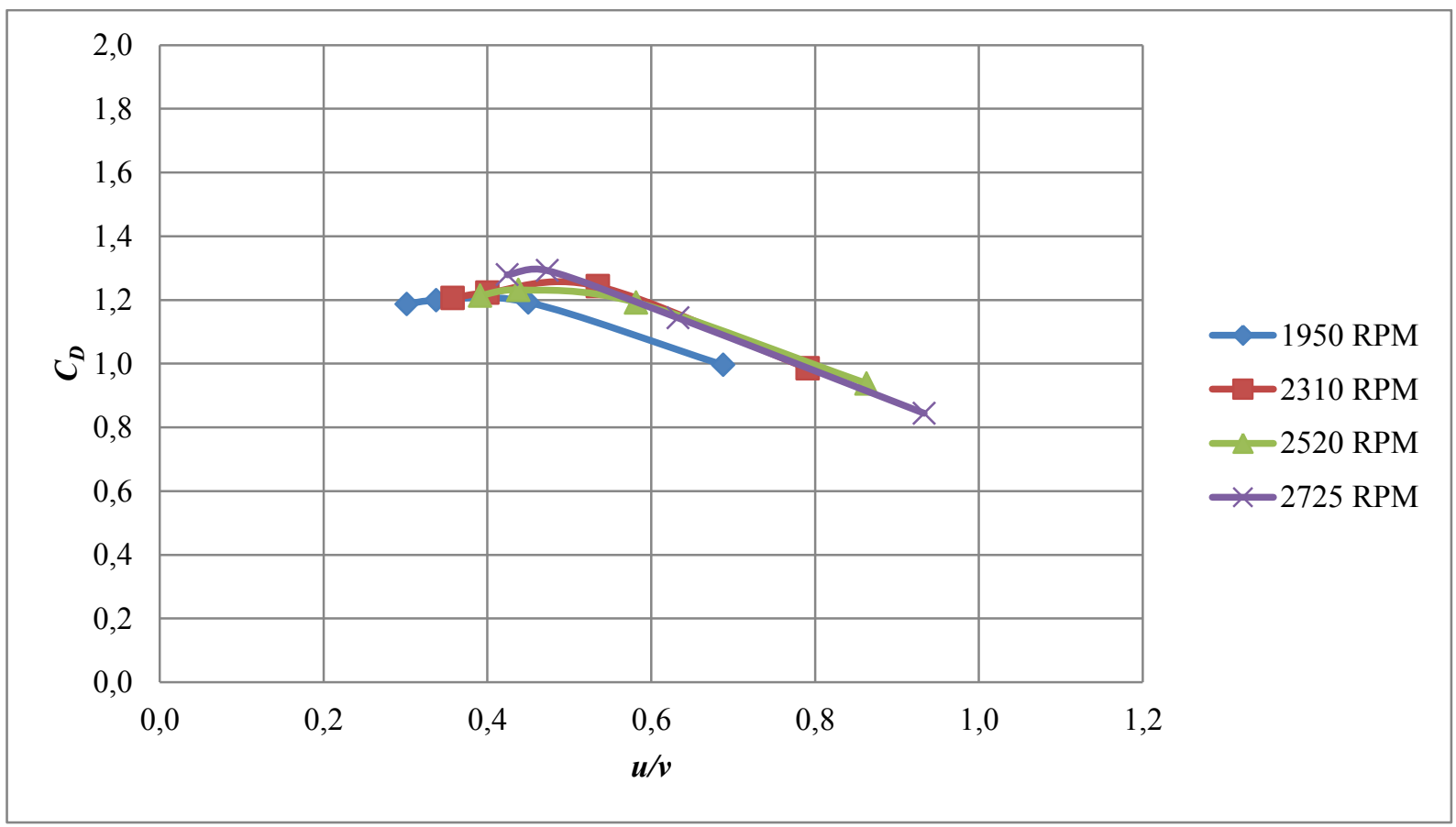

Figura A1.52 Variación del coeficiente de resistencia $C_{\boldsymbol{D}}$ en función de la relación de velocidades $u / v$ para cada velocidad de giro $\omega$. 


\subsubsection{Placa plana fina}

Los resultados correspondientes a los ensayos de sustentación y resistencia se presentan en la tabla A1.15.

Tabla A1.15 Resultados correspondiente a los ensayos de sustentación y resistencia.

\begin{tabular}{|c|c|c|c|c|c|c|c|c|}
\hline \multicolumn{9}{|c|}{ Placa plana fina $(\phi=0,035 \mathrm{~m})$} \\
\hline $\begin{array}{c}\omega \\
{[\mathbf{r p m}]}\end{array}$ & $\begin{array}{c}v \\
{[\mathbf{m} / \mathbf{s}]}\end{array}$ & $\begin{array}{c}L \\
{[\mathbf{N}]}\end{array}$ & $C_{L}$ & $u / v$ & $\begin{array}{c}v \\
{[\mathbf{m} / \mathbf{s}]}\end{array}$ & $\begin{array}{c}D \\
{[\mathbf{N}]}\end{array}$ & $C_{D}$ & $u / v$ \\
\hline \multirow[t]{4}{*}{0} & - & - & - & - & 5,07 & 0,18 & 1,88 & 0,00 \\
\hline & - & - & - & - & 7,83 & 0,42 & 1,81 & 0,00 \\
\hline & - & - & - & - & 10,34 & 0,73 & 1,82 & 0,00 \\
\hline & - & - & - & - & 11,59 & 0,92 & 1,83 & 0,00 \\
\hline \multirow[t]{4}{*}{1950} & 5,15 & 0,16 & 1,57 & 0,69 & 5,15 & 0,13 & 1,31 & 0,69 \\
\hline & 7,89 & 0,28 & 1,21 & 0,45 & 7,83 & 0,30 & 1,31 & 0,46 \\
\hline & 10,55 & 0,45 & 1,09 & 0,34 & 10,38 & 0,50 & 1,24 & 0,34 \\
\hline & 11,81 & 0,54 & 1,04 & 0,30 & 11,66 & 0,66 & 1,30 & 0,31 \\
\hline \multirow[t]{4}{*}{2310} & 5,15 & 0,17 & 1,67 & 0,82 & 5,15 & 0,13 & 1,31 & 0,82 \\
\hline & 7,89 & 0,31 & 1,34 & 0,54 & 7,83 & 0,31 & 1,36 & 0,54 \\
\hline & 10,55 & 0,47 & 1,14 & 0,40 & 10,38 & 0,53 & 1,32 & 0,41 \\
\hline & 11,81 & 0,56 & 1,08 & 0,36 & 11,66 & 0,68 & 1,33 & 0,36 \\
\hline \multirow[t]{4}{*}{2520} & 5,19 & 0,18 & 1,74 & 0,89 & 5,15 & 0,13 & 1,26 & 0,90 \\
\hline & 7,89 & 0,32 & 1,38 & 0,59 & 7,83 & 0,31 & 1,36 & 0,59 \\
\hline & 10,53 & 0,49 & 1,18 & 0,44 & 10,42 & 0,53 & 1,30 & 0,44 \\
\hline & 11,81 & 0,58 & 1,12 & 0,39 & 11,69 & 0,67 & 1,31 & 0,39 \\
\hline \multirow[t]{4}{*}{2725} & 5,19 & 0,18 & 1,79 & 0,96 & 5,19 & 0,13 & 1,29 & 0,96 \\
\hline & 7,89 & 0,34 & 1,45 & 0,63 & 7,86 & 0,31 & 1,35 & 0,64 \\
\hline & 10,55 & 0,52 & 1,26 & 0,47 & 10,42 & 0,54 & 1,32 & 0,48 \\
\hline & 11,81 & 0,61 & 1,18 & 0,42 & 11,66 & 0,67 & 1,32 & 0,43 \\
\hline
\end{tabular}




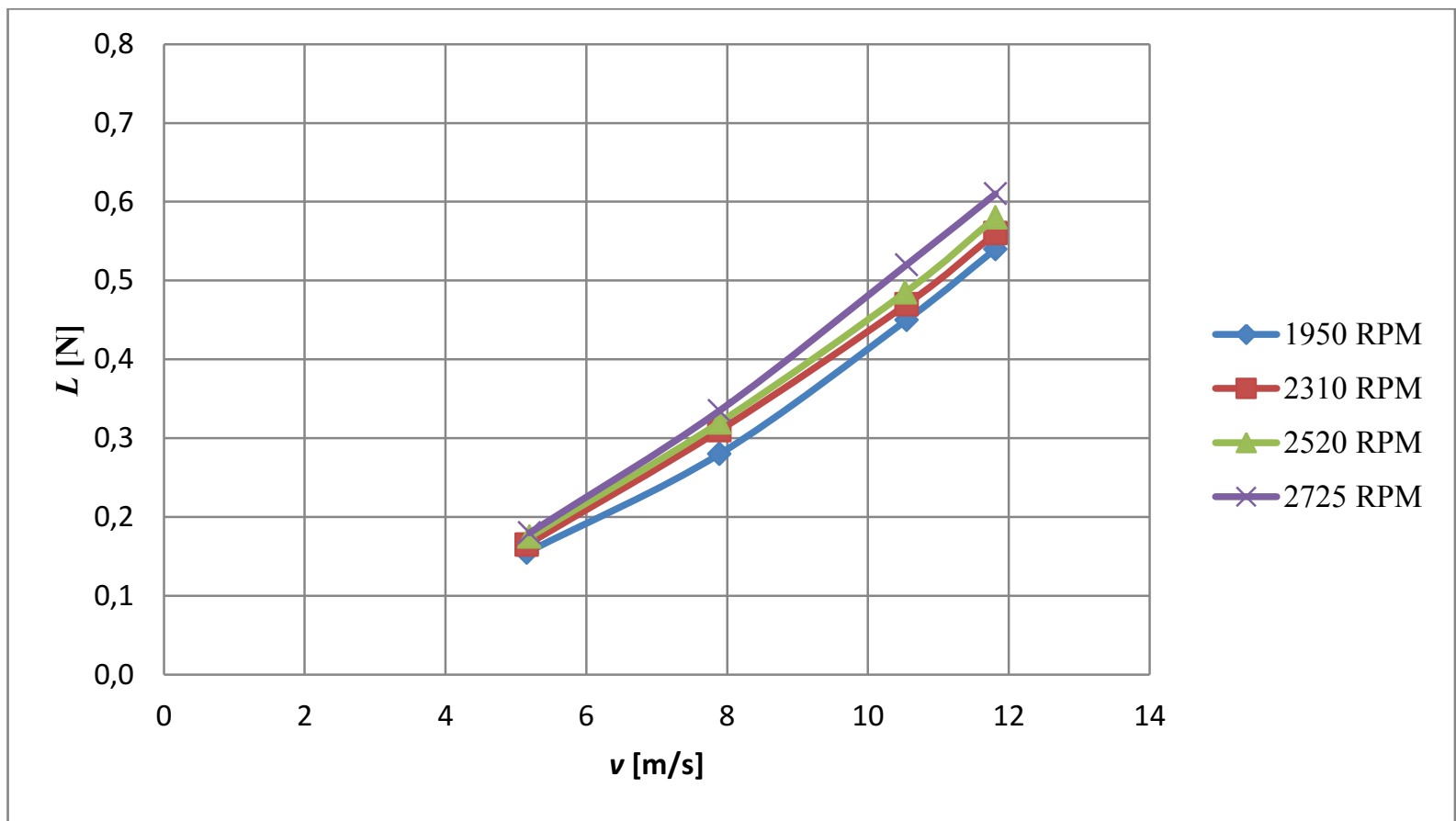

Figura A1.53 Variación de la sustentación $L$ en función de la velocidad de la corriente $v$ para cada velocidad de giro $\omega$.

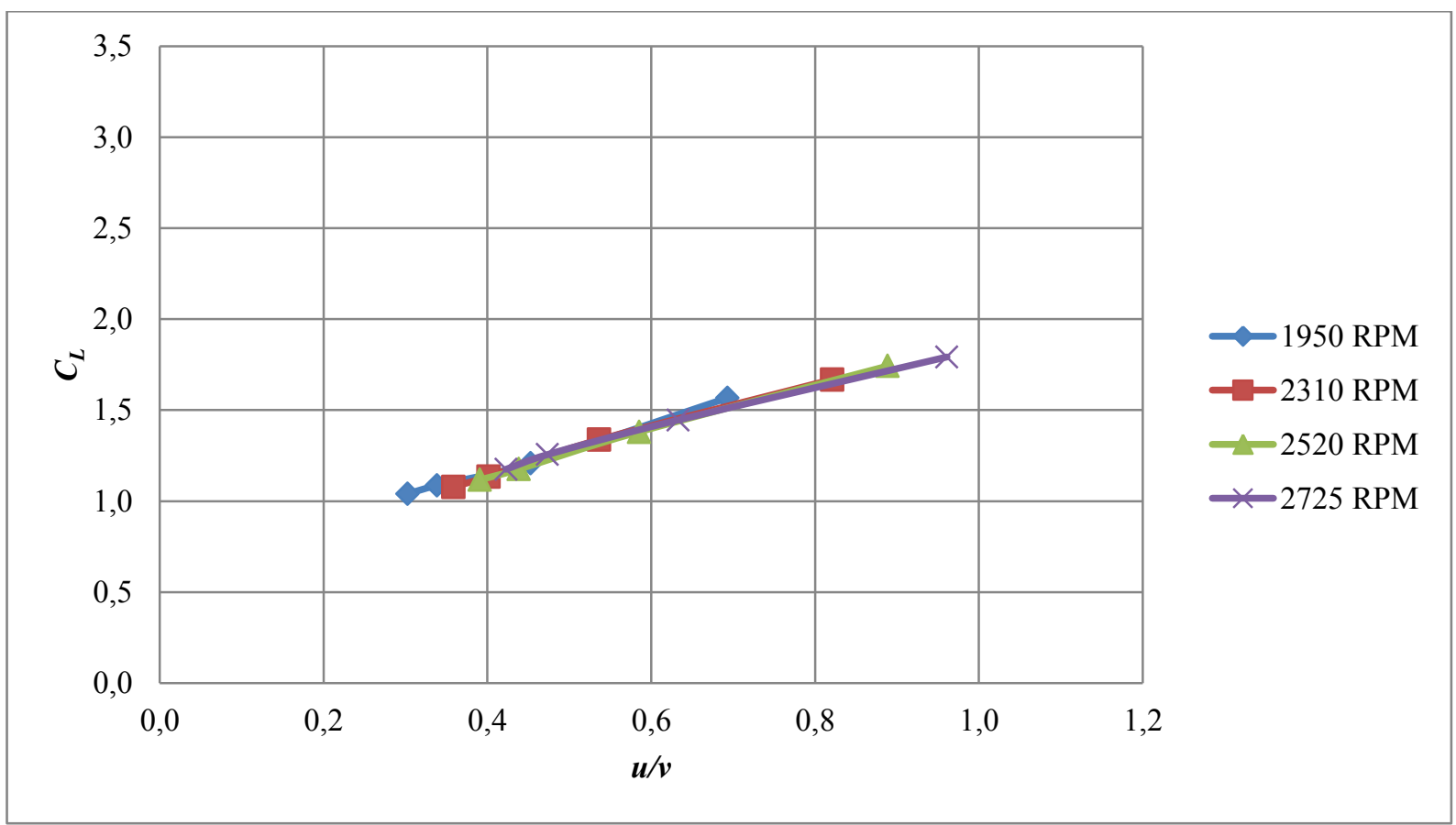

Figura A1.54 Variación del coeficiente de sustentación $C_{L}$ en función de la relación de velocidades $u / v$ para cada velocidad de giro $\omega$. 


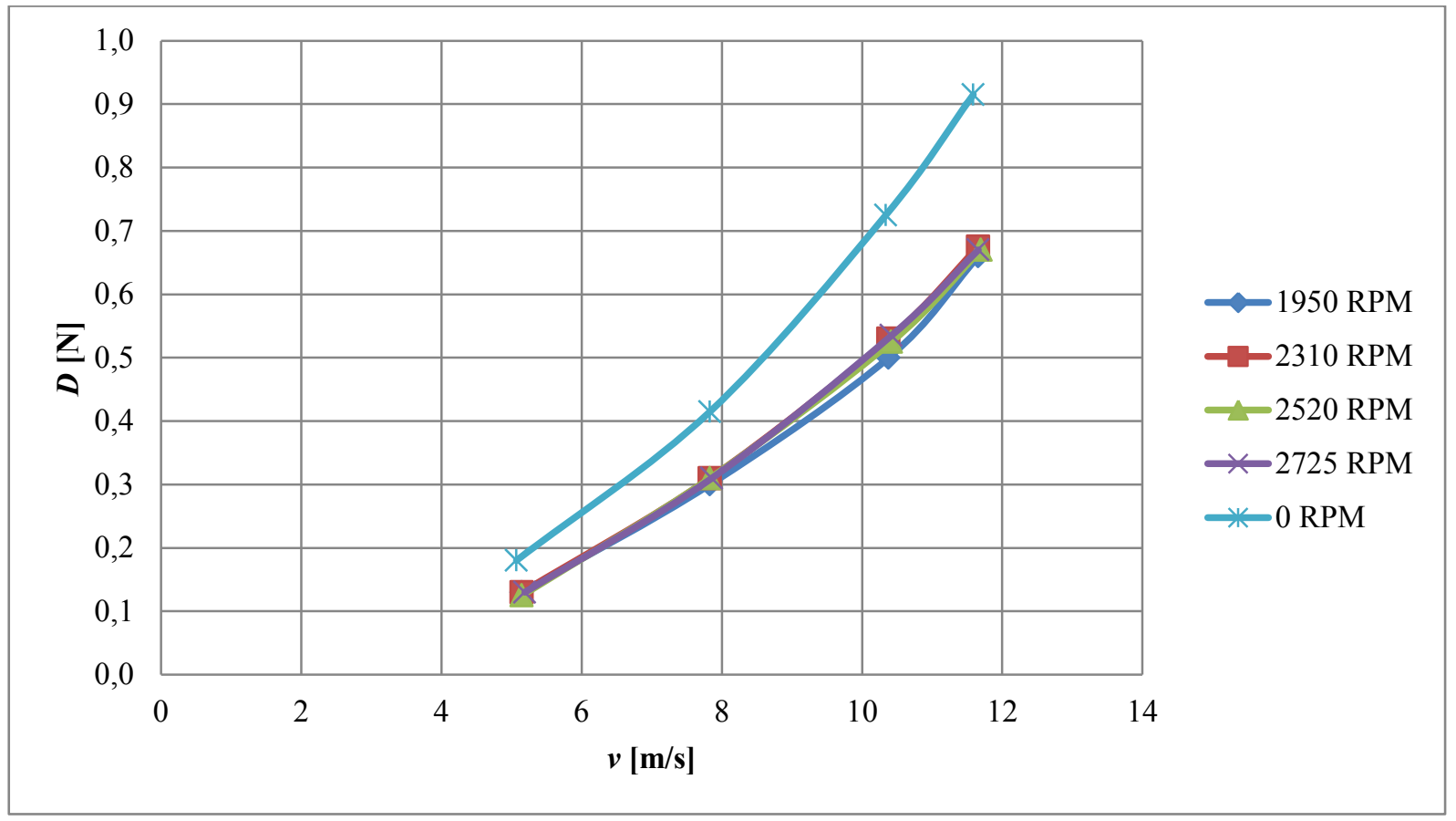

Figura A1.55 Variación de la resistencia $D$ en función de la velocidad de la corriente $v$ para cada velocidad de giro $\omega$.

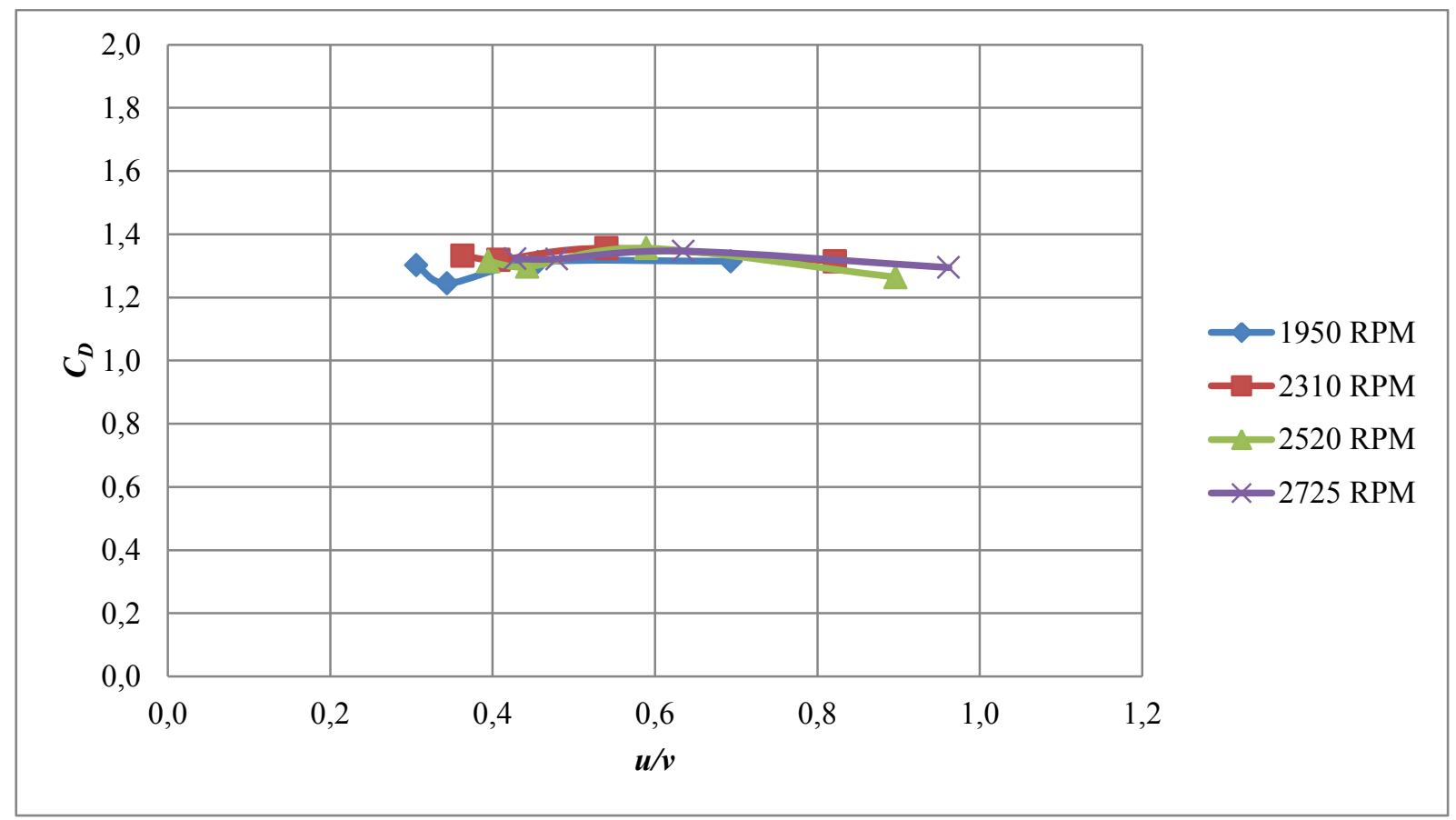

Figura A1.56 Variación del coeficiente de resistencia $C_{\boldsymbol{D}}$ en función de la relación de velocidades $u / v$ para cada velocidad de giro $\omega$. 


\section{ANEXO 2}

\section{CALCULO DE INCERTIDUMBRES EN LAS MEDICIONES}

\subsection{INTRODUCCIÓN}

En el presente anexo se calcula la incertidumbre para la determinación de los coeficientes aerodinámicos $C_{L}$ y $C_{D}$, y de la relación de velocidades $u / v$ para los ensayos experimentales realizados.

\subsection{DESARROLLO}

En el caso del coeficiente de sustentación $C_{L}$ (A2.1) las fuentes de incertidumbre se presentan por la determinación de los siguientes parámetros:

- La sustentación, $L$.

- La densidad del aire, $\rho$.

- La velocidad de la corriente, $v$.

- La superficie del cuerpo, $S$.

$$
C_{L}=\frac{L}{\frac{1}{2} \rho v^{2} S}
$$

La incertidumbre $U_{C L}$ se calcula a partir de la expresión (A2.2):

$$
U_{C_{L}}^{2}=U_{L}^{2}\left(\frac{\partial C_{L}}{\partial L}\right)^{2}+U_{\rho}^{2}\left(\frac{\partial C_{L}}{\partial \rho}\right)^{2}+U_{v}^{2}\left(\frac{\partial C_{L}}{\partial v}\right)^{2}+U_{S}^{2}\left(\frac{\partial C_{L}}{\partial S}\right)^{2}
$$

donde, 
$\frac{\partial C_{L}}{\partial L}=\frac{1}{\frac{1}{2} \rho v^{2} S}$

$\frac{\partial C_{L}}{\partial \rho}=\frac{L}{\frac{1}{2} v^{2} S}\left(-\frac{1}{\rho^{2}}\right)$

$\frac{\partial C_{L}}{\partial v}=\frac{L}{\frac{1}{2} \rho S}\left(-\frac{1}{v^{3}}\right)$

$\frac{\partial C_{L}}{\partial S}=\frac{L}{\frac{1}{2} \rho v^{2}}\left(-\frac{1}{S^{2}}\right)$

Al igual que en el caso del $C_{L}$, el $C_{D}$ se determina con la expresión (A2.7) y las fuentes de incertidumbre se presentan por la determinación de los siguientes parámetros:

- La resistencia, $D$.

- La densidad del aire, $\rho$.

- La velocidad de la corriente, $v$.

- La superficie del cuerpo, $S$.

$C_{D}=\frac{D}{\frac{1}{2} \rho v^{2} S}$

La incertidumbre $U_{C D}$ se calcula a partir de la expresión (A2.8):

$U_{C_{D}}^{2}=U_{D}^{2}\left(\frac{\partial C_{D}}{\partial D}\right)^{2}+U_{\rho}^{2}\left(\frac{\partial C_{D}}{\partial \rho}\right)^{2}+U_{v}^{2}\left(\frac{\partial C_{D}}{\partial v}\right)^{2}+U_{S}^{2}\left(\frac{\partial C_{D}}{\partial S}\right)^{2}$

donde,

$\frac{\partial C_{D}}{\partial D}=\frac{1}{\frac{1}{2} \rho v^{2} S}$ 


$$
\begin{aligned}
& \frac{\partial C_{D}}{\partial \rho}=\frac{D}{\frac{1}{2} v^{2} S}\left(-\frac{1}{\rho^{2}}\right) \\
& \frac{\partial C_{D}}{\partial v}=\frac{D}{\frac{1}{2} \rho S}\left(-\frac{1}{v^{3}}\right) \\
& \frac{\partial C_{D}}{\partial S}=\frac{D}{\frac{1}{2} \rho v^{2}}\left(-\frac{1}{S^{2}}\right)
\end{aligned}
$$

Para relación de velocidades $u / v$, la misma se determina a partir de la expresión (A2.13) donde las fuentes de incertidumbre se presentan por la determinación de los siguientes parámetros:

- La velocidad de giro, $\omega$.

- El radio del cuerpo, $R$.

- La velocidad de la corriente, $v$.

$\frac{u}{v}=\frac{\omega R}{v}$

La incertidumbre $U_{u / v}$ se calcula a partir de la expresión (A2.14):

$U_{\frac{u}{v}}^{2}=U_{\omega}^{2}\left(\frac{\partial \frac{u}{v}}{\partial \omega}\right)^{2}+U_{v}^{2}\left(\frac{\partial \frac{u}{v}}{\partial v}\right)^{2}+U_{R}^{2}\left(\frac{\partial \frac{u}{v}}{\partial R}\right)^{2}$

donde,

$\frac{\partial \frac{u}{v}}{\partial \omega}=\frac{R}{v}$

$\frac{\partial \frac{u}{v}}{\partial v}=\omega R\left(-\frac{1}{v^{2}}\right)$ 
$\frac{\partial \frac{u}{v}}{\partial R}=\frac{\omega}{v}$

\subsection{RESULTADOS}

En el presente estudio se analizaron todos los cuerpos ensayados, ya que cambian las condiciones de sustentación $L$, resistencia $D$, velocidad de giro $\omega$ y los diámetros de referencia $\phi$ de los cuerpos.

Como fuentes de contribución de incertidumbre de cada instrumento se tomaron: para la balanza, el tacómetro y el calibre ,las incertidumbres de resolución; para la medición de la velocidad se utilizó el procedimiento para la determinación de la incertidumbre en los ensayos de calibración de anemómetros, ANE-IT-001/002, desarrollado por la UIDET GTA GIAI de la Facultad de Ingeniería de la UNLP, el cual tiene en cuenta las incertidumbres de resolución y calibración del micromanómetro, resolución de los termómetros de bulbo seco y húmedo, y del barómetro.

A modo de ejemplo, en la tabla A2.1. se presentan los resultados de la determinación de la incertidumbre en el cálculo de $C_{L}$ y $C_{D}$ utilizando el cuerpo cuadrado.

Tabla A2.1 Incertidumbre para la determinación de $C_{L}$ y $C_{D}$ en función de la velocidad $v$.

\begin{tabular}{|c|c|c|c|c|}
\hline $\begin{array}{c}v \\
{[\mathrm{~m} / \mathrm{s}]}\end{array}$ & $U_{C L}$ & $\begin{array}{c}U_{C L} \\
{[\%]}\end{array}$ & $U_{C D}$ & $\begin{array}{c}U_{C D} \\
{[\%]}\end{array}$ \\
\hline 5 & 0,036 & 2,0 & 0,028 & 2,0 \\
\hline 7 & 0,022 & 2,1 & 0,029 & 2,0 \\
\hline 10 & 0,014 & 1,6 & 0,023 & 1,7 \\
\hline 12 & 0,011 & 1,4 & 0,020 & 1,4 \\
\hline
\end{tabular}

En la tabla A2.2. se presentan los resultados de la determinación de la incertidumbre en el cálculo de $u / v$ utilizando como ejemplo el cuerpo cuadrado para una velocidad de giro $\omega=1950 \mathrm{rpm}$. 
Tabla A2.2 Incertidumbre para la determinación de $u / v$ en función de la velocidad $v$.

\begin{tabular}{|c|c|c|}
\hline $\begin{array}{c}v \\
{[\mathrm{~m} / \mathrm{s}]}\end{array}$ & $U u / v$ & $\begin{array}{c}U u / v \\
{[\%]}\end{array}$ \\
\hline 5 & 0,007 & 0,92 \\
\hline 7 & 0,004 & 0,70 \\
\hline 10 & 0,002 & 0,55 \\
\hline 12 & 0,002 & 0,50 \\
\hline
\end{tabular}

Analizando el resto de los cuerpos para todas las condiciones de ensayo se observa que los valores de incertidumbre para todo el conjunto de modelos para $C_{L}$ y $C_{D}$ varían desde $1,4 \%$ hasta un 3,2\%, mientras que para $u / v$ varían entre el $0,5 \%$ y $1,2 \%$. 


\section{BIBLIOGRAFÍA}

[1] Seifert, J., "A review of the Magnus Effect in Aeronautics". Progress in aerospace sciences, 55, p: $17-45.2012$.

[2] Betz, A., "The Magnus effect - the principle of the Flettner rotor", Technical Memorandums National Advisory Committee For Aeronautics. No. 310. 1925.

[3] Prandtl, L., "Application of the Magnus Effect to the Wind Propulsion of Ships". Technical Memorandums National Advisory Committee For Aeronautics. No. 367. 1926.

[4] Ahlborn, F. "The Magnus Effect in Theory and in Reality", Technical Memorandums National Advisory Committee For Aeronautics. No. 567. 1929.

[5] Fletcher, C. "Negative Magnus Forces in the Critical Reynolds Number Regime". Journal of Aircraft. Vol. 9. No. 12, p: $826-834.1972$.

[6] Muto, M., Watanabe, H., Tsubokura, M., Oshima, N. "Negative Magnus Effect on a Rotating Sphere at around the Critical Reynolds Number". 13th European Turbulence Conference. Journal of Physics: Conference Series 318, p: 1 - 9. 2011.

[7] Miller, C. "Wind Tunnel Measurement of the Surface Pressure Distribution on a Spinning Magnus Rotor”. Journal of Aircraft. Vol. 16. No. 12, p: 815 - 822. 1979.

[8] Leech, F. "The Stability of Six Degree of Freedom Dynamics with Magnus Effect". Journal of the Franklin Institute. Pergamon Press Ltd. Vol. 309. No. 2, p: $65-90$. 1980.

[9] Howerton, W. "Experimental Investigation of the Aerodynamics of Independently Rotating Cylindrical Shells". No. AFIT/GAE/ENY/92D-11. Air Force Institute of Technology. Wright - Patterson AFB OH School of Engineering. 1993.

[10] Cuevas, J. C., Ocaña, O., Hurtado, A., \& Hidalgo, S. "El efecto Magnus y La paradoja de D'Alembert: consideraciones del flujo Potencial." Lat. Am. J. Phys. Educ. Vol. 4. No. 2, p: 394 - 398. 2010.

[11] Rahman, A. S. S. "Flow around a rotating short cylinder: a computational study" Thesis in Mechanical Engineering. 1996.

[12] Mittal, S., Kummar, B. "Flow Past a rotating cylinder". Journal of Fluid Mechanics. Vol. 476, p: 303 - 334. 2002. 
[13] Gowree, E. R., Prince S. A. "A Computational study of the aerodynamics of a spinning cylinder in a crossflow of high Reynolds number". 28th International Congress of the Aeronautical Sciences. 2012.

[14] Ericcson, L. E., Beyers M. E. "Flat Spin of a Circular Cylinder". Journal of Aircraft. Vol. 33. No. 4, p: 722 - 729. 1996.

[15] Ericcson, L.E. “Circular Cylinder Response to Kármán Vortex Shedding”. Journal of Aircraft. Vol. 25. No. 9, p: 769 - 775. 1988.

[16] Polhamus, E., "Effect of flow incidence and Reynolds number on low speed aerodynamic characteristics of several noncircular cylinders with applications to directional stability and spinning". Technical Note National Advisory Committee for Aeronautics. No. 4176. 1958.

[17] Pamadi, B.N., Pordal, H.S. "Effect of Strakes on the Autorotational Characteristics of Noncircular Cylinders”. Journal of Aircraft. Vol. 22. No. 2, p: 84 - 97. 1987.

[18] Skews, B.W., "Autorotation of many-sided bodies in an airstream". Letters to nature. Vol 352, p: 512 - 513. 1991.

[19] Zaki, T.G., Sen M., Gad El Hak M. "Numerical and Experimental Investigation of Flow Past and Freely Rotable Square Cilinder". Journal of Fluids and Structures. No. 8, p: 555 - 582. 1994.

[20] Ozgoren, M., "Flow structure in the downstream of square and circular cylinders". Flow Measurement and Instrumentation 17, p: 225 - 235. 2006.

[21] Lysenko, D. A., Ertesvag I. S., Rian K. E. "Modeling of Turbulent separated flows using OpenFoam". Computers \& Fluids. p: 1 - 15. 2012.

[22] Queijo, M. J., Fletcher, Herman S. "Low-speed experimental investigation of the Magnus effect on various sections of a body of revolution with and without a propeller". Technical Memorandums National Advisory Committee For Aeronautics. No. 4013. 1957.

[23] Jacobson, I.D., "Magnus Characteristics of Arbitrary Rotating Bodies". Advisory Group of Aerospace Research \& Development. No. 171. 1973.

[24] Platou, A. S., "The Magnus Force on a short body at supersonic speed". Ballistic Division Aerodynamics Branch. Report No. 1062. 1959.

[25] Ericcson, L.E., "Wing Rock Generated by Forebodie Vortices". Journal of Aircraft. Vol. 26. No. 2, p: 110 - 116. 1989.

[26] Swanson, W. M., “The Magnus Effect: A Summary of Investigations to Date”, Journal of Fluids Engineering. Vol. 83, p: 461 - 470. 1961. 
[27] Iversen, J. "Correlation of Magnus force data for slender spinning cylinders" Journal of Spacecraft and Rockets. Vol. 10. No. 4, p: 268. 1973.

[28] Power, H., Iversen J. "Magnus Effect on Spinning Bodies of Revolution". AIAA Journal. Vol. 11. No. 4, p: 417 - 418. 1973.

[29] Power, H. “Spinning Missile Magnus Force Measurement”. Technical Report of Naval Postgraduate School Monterrey California. 1974.

[30] Power Jr., H. L. "Boundary layer contribution to the Magnus effect on a spinning cylinder at low angles of attack". A Dissertation Submitted to the Graduate Faculty in Partial Fulfillment of The Requirements for the Degree of Doctor of Philosofy. Iowa State University. 1971.

[31] Kelly, H. R. "The Estimation of Normal Force and Pitching Moment Coefficients for Blunt - Based Bodies of Revolution at Large Angle of Attack". Ballistic Division Aerodynamics Branch. Local Proyect 701. 1953.

[32] Beard, T. R. "An Investigation of the Production of Magnus Forces on a High Fineness Ratio Body of Revolution". Naval Postgraduate School. Monterrey, California. 1973.

[33] Milton, J. E. "Method for Calculating Magnus Forces on Slender Bodyes of Revolution”. Technical Report AFATL-TR-74-51. University of Florida. 1974.

[34] Wang, K. C. "Boundary Layer over spinning Blunt - Body of revolution at incidence and Magnus Forces”. MML TR 77-50c. Martin Marietta Laboratories. 1977.

[35] Modi, V.J., Sun J.L.C, Akutsu T., Lake, P., McMillan K., Swinton P.G., Mullinst D. "Moving-Surface Boundary-Layer Control for Aircraft Operation at High Incidence". Journal of Aircraft. Vol. 18. No. 11, p: 963 - 968. 1981.

[36] Ericcson, L.E. "Dynamic Airfoil Flow Separation and Reattachment". Journal of Aircraft. Vol. 32. No. 6, p: $1191-1197.1995$.

[37] Al Garni, A. Z., Al Garni, A. M, Ahmed, S.A., Sahin, A.Z, "Flow Control for an Airfoil with Leading Edge Rotation: An experimental study". Journal of Aircraft. Vol. 37. No. 4, p: $617-622.2000$.

[38] Kano, I., Yagita M. "Flow around a RotatingCilircular Cylinder near a Moving PlaneWall”. JSME International Journal. Serie B. Vol. 45. No. 2, p: 259 - 268. 2002.

[39] Curtiss, H.C., Putman, W.F. "Stability and Control Characterístics of the Aerocrane Hybrid Heavy - Lift Vehicle". Journal of Aircraft. Vol. 17. No. 10, p: 719 - 726. 1980.

[40] Bahill, A. T., Bladwin, D. G., Ramberg, J. S. "Effects of Altitude and Atmosferic Conditions on the flight of a Bsseball". International Journal of Sports Science and Engineering. Vol. 3. No. 2, p: 109 - 128. World Academic Press, World Academic Union. 2009. 
[41] Alaways, L., Hubbard, M. "Experimental determination of baseball spin and lift". Journal of Sport Sciences. Vol. 19, p: 349 - 358. 2001.

[42] Barber, S., Chin S. B., Carré M. J. "Sports ball aerodynamics: A numerical study of the erratic motion of soccer balls". Computers \& Fluids. 38, p: 1091 - 1100. 2009.

[43] Tai, C., Chao, C., Leong, J., Hong, Q., "Effects of golf ball dimple configuration on aerodynamics, trajectory, and acoustics". Journal of Flow Visualization and Image Processing. Vol. 14. 2007.

[44] Kray, T., Franke, J., Frank, W., "Magnus Effect on a rotating sphere at high Reynolds numbers". Journal of Wind Engineering and Industrial Aerodynamics. 110, p: $1-9$. 2012.

[45] Minami, S., Azuma, A. "Various flying modes of wind-dispersal seds". Journal of Theoretical Biology. 225, p: 1 - 14. 2003.

[46] Nguyen, S. N., Greenhalgh E. S., Olsson R., Iannucci L. "Modeling the lofting of Runway Debris by Aircraft Tyres". Journal of Aircraft. Vol. 45.No. 5, p: $1701-1714$. 2008 .

[47] Holmes, J.D. "Trajectories of spheres in strong winds with application to wind-borne debris". Journal of Wind Engineering and Industrial Aerodynamics. 92, p: $9-22$. 2004.

[48] Lin, N., Letchford C., Holmes J.D. "Investigation of plate-type windborne debris. Part I: Experiments in wind tunnel and full scale". Journal of Wind Engineering and Industrial Aerodynamics. 94, p: 51 - 76. 2006.

[49] Holmes, J.D., Letchford C. and Lin N. "Investigations of plate-type windborne debris. Part II: Computed Trayectories". Journal of Wind Engineering and Industrial Aerodynamics. 94, p: $21-39.2007$.

[50] Baker, C. J. "The Debris Flight Equations". Journal of Wind Engineering and Industrial Aerodynamics. 95, p: 329 - 353. 2007.

[51] Visscher, B. T., Kopp G. A. "Trajectories of roof sheating panels under high winds". Journal of Wind Engineering and Industrial Aerodynamics. 95, p: 697 - 713. 2007.

[52] Richards, P. J., Williams N., Laing B., Mc Carthy M., Pond M. "Numerical Calculation of the three dimensional motion of wind - borne debris". Journal of Wind Engineering and Industrial Aerodynamics, 96, p: 2188 - 2202. 2008.

[53] Kakimpa, B., Hargreaves D., Owen J. S. "The flight of wind borne debris: an experimental, analytical and numerical investigation - Part III: CFD simulations". The Seventh Asia-Pacific Conference on Wind Engineering. 2009. 
[54] Kakimpa, B., Hargreaves D. M., Owen J. S. "An Investigation of plate - type windborne debris flight using coupled CFD - RBD models. Part I: Model developmet and validation". Journal of Wind Engineering and Industrial Aerodynamics. 111, p..104 - 116. 2012.

[55] Kakimpa, B., Hargreaves D. M., Owen J. S. "An Investigation of plate - type windborne debris flight using coupled CFD - RBD models. Part II: Free and Constrained flight". Journal of Wind Engineering and Industrial Aerodynamics. 111, p: $95-103.2012$.

[56] Hirata, K., Shimizu K., Fukuhara K., Yamauchi K., Kawaguchi D., Funaki J. "Aerodynamic Characteristics of a Tumbling Plate under Free Flight". Journal of Fluid Science and Technology. Vol. 4. No. 1, p: 168 - 187. 2009.

[57] Kordi, B., Kopp G. A. "Effects of initial conditions on the flight of windborne plate debris". Journal of Wind Engineering and Industrial Aerodynamics. 99, p: $601-614$. 2011.

[58] Maruyama, T., "Simulation of flying debris using a numerically generated tornadolike vortex". Journal of Wind Engineering and Industrial Aerodynamics. 99, p: 249 256. 2011.

[59] Jhonson, W., "The Magnus Effect - Early Investigations and a questions of priority". International Journal of Mechanical Sciencies. Vol. 28. No. 12, p: 859 - 872. 1986.

[60] Tokumaru, P., Dimotakis, P. "The lift of a cylinder executing rotary motions in a uniform flow". Journal of Fluid Mechanics. Vol. 255, p: 1 - 10. 1993.

[61] Weidman, P., Herczynski, A. "On the inverse Magnus Effect in free molecular Flow". Physics of Fluids. Vol. 16. No. 2, p: L9 - L12. 2004.

[62] Volkov, A., "Aerodynamic Coefficients of a Spinning Sphere in a Rarefied-Gas Flow". Fluid Dynamics. Vol. 44. No.1, p: 141 - 157. 2009.

[63] Pope, A., "Wind Tunnel Testing". Jhon Wiley \& Sons, Inc. Chapman \& Hall, Limited. London. 1947.

[64] "Wind Tunnel Wall Correction". AGARD AG336. North Atlantic Treaty Organization 1998.

[65] Glauert, H., "Wind Tunnel Interference on Wings, Bodies and Airscrews". Aeronautical Research Commitee - Reports and Memoranda. No. 1566. 1933.

[66] Ross, I., Altman, A., "Wind tunnel blockage corrections: Review and application to Savonius vertical-axis wind turbines". Journal of Wind Engineering and Industrial Aerodynamics. No. 99, p: 523 - 538. 2011.

[67] Alexander, A. J., Holownia, B. P. "Wind Tunnel Tests on a Savonius Rotor". Journal of Industrial Aerodynamics. No. 3, p: 343 - 351. 1978. 
[68] Ackeret, J., "Recent Experiments at the Gottingen Aerodinamic Institute". Technical Memorandums National Advisory Committee For Aeronautics. No. 323. 1925.

[69] Reid, E. C., “Test of rotating cylinders". Technical Memorandums National Advisory Committee For Aeronautics. No. 209. 1924.

[70] Skews, B. W., "Autorotation of Polygonal prisms with an upstream vane". Journal of Wind Engineering and Industrial Aerodynamics. No. 73, p: 145 - 158. 1997.

[71] Tachikawa, M., "Trajectories of flat plates in uniform flow with applications to windgenerated missiles", Journal of Wind Engineering and Industrial Aerodynamics. No. 14, p: 443 - 453. 1983.

[72] Badalamenti, C., "On the application of rotating cylinders to micro air vehicles". Tesis Doctoral. City University. 2010.

[73] Takayama, S., Aoki, K. "Flow Characteristics around a Rotating Grooved Circular Cylinder with Grooves of Different Depths", Journal of Visualization, Vol. 8, p: 295 303.2005 . 